Geoacoustic Inversion in Laterally Varying Shallow-Water Environments Using High-Resolution Wavenumber Estimation by

Kyle M. Becker

B.S., Mechanical Engineering, Boston University (1991)

S.M., Acoustics, The Pennsylvania State University (1995)

\author{
Submitted in partial fulfillment of the \\ requirements for the degree of \\ Doctor of Philosophy in Applied Ocean Sciences \\ at the \\ MASSACHUSETTS INSTITUTE OF TECHNOLOGY \\ and the \\ WOODS HOLE OCEANOGRAPHIC INSTITUTION \\ February 2002
}

(C)Kyle M. Becker, 2001.

The author hereby grants to MIT and WHOI permission to reproduce and to distribute copies of this thesis in whole or in part.

Author .

Department of Applied Ocean Physics and Engineering, WHOI Department of Ocean Engineering, MIT

February, 2002

Certified by ...................

George V. Frisk Senior Scientist, WHOI Thesis Supervisor

Accepted by

Michael S. Triantafyllou Chair, Joint Committee in Applied Ocean Science and Engineering, MIT/WHOI 


\title{
Geoacoustic Inversion in Laterally Varying Shallow-Water Environments Using High-Resolution Wavenumber
}

\author{
Estimation
}

by

Kyle M. Becker

\author{
Submitted to the Department of Applied Ocean Physics and Engineering, WHOI \\ Department of Ocean Engineering, MIT \\ on February, 2002, in partial fulfillment of the \\ requirements for the degree of \\ Doctor of Philosophy in Oceanographic Engineering
}

\begin{abstract}
Sound propagation in shallow water is highly dependent on the interaction of the sound field with the bottom. In order to fully understand this problem, it is necessary to obtain reliable estimates of bottom geoacoustic properties that can be used in acoustic propagation codes. In this thesis, perturbative inversion methods and exact inverse methods are discussed as a means for inferring geoacoustic properties of the bottom. For each of these methods, the input data to the inversion is the horizontal wavenumber spectrum of a point-source acoustic field. The main thrust of the thesis work concerns extracting horizontal wavenumber content for fully threedimensionally varying waveguide environments. In this context, a high-resolution autoregressive (AR) spectral estimator was applied to determine wavenumber content for short aperture data. As part of this work, the AR estimator was examined for its ability to detect discrete wavenumbers in the presence of noise and also to resolve closely spaced wavenumbers for short aperture data. As part of a geoacoustic inversion workshop, the estimator was applied to extract horizontal wavenumber content for synthetic pressure field data with range-varying geoacoustic properties in the sediment. The resulting wavenumber content was used as input data to a perturbative inverse algorithm to determine the sound speed profile in the sediment. It was shown using the high-resolution wavenumber estimator that both the shape and location of the range-variability in the sediment could be determined. The estimator was also applied to determine wavenumbers for synthetic data where the water column sound speed contained temporal variations due to the presence of internal waves. It was shown that reliable estimates of horizontal wavenumbers could be obtained that are consistent with the boundary conditions of the waveguide. The Modal
\end{abstract}


Mapping Experiment (MOMAX), an experimental method for measuring the full spatial variability of a propagating sound field and its corresponding modal content in two-dimensions, is also discussed. The AR estimator is applied to extract modal content from the real data and interpreted with respect to source/receiver motion and geometry. For a moving source, it is shown that the wavenumber content is Doppler shifted. A method is then described that allows the direct measure of modal group velocities from Doppler shifted wavenumber spectra. Finally, numerical studies are presented addressing the practical issues associated with using MOMAX type data in the exact inversion method of Gelfand-Levitan.

Thesis Supervisor: George V. Frisk

Title: Senior Scientist, WHOI 


\section{Acknowledgments}

I'd like to thank my thesis advisor, George V. Frisk, for guiding my thesis work, and also for taking an interest in my professional development in the field of underwater acoustics. I'd also like to thank Jim Preisig and Henrik Schmidt for their insight and encouragement. I owe a debt of gratitude to Joyce McLaughlin of RPI for taking an interest in my work and giving me a new perspective. Also, although not part of my committee, Subramaniam Rajan played a major role in helping me to understand and implement the perturbative inversion schemes. He was also an excellent host, allowing me to come work with him in at his offices in Seattle and also New Hampshire on several occasions.

I am especially grateful to ONR for providing the funding for me to do this work. The education office was also very generous in filling in the gaps to provide funding for travel and other niceties. Julia, Marsha, Marcey, Stacey, and Stella made themselves available for any inquiry and seemed to always say yes to any request.

I want to thank Cynthia Sellers and Jim Doutt for both their professional help and their friendship. Arthur Newhall was also exceptionally giving with his time and expertise. Keith von Der Heydt, Cal Eck, and Neil McPhee, were instrumental in making sure the experiments went off and that I was provided with excellent data for this work.

When I wasn't working, Sean McKenna and Joe Warren were great allies in both fun and commiseration. Susan and Gordon, and their kids Colin and Aidan also provided welcome escape from work when it was needed the most. My parents, Paul and Eileen Becker, along with my brothers and sisters were also always encouraging during the course of my studies.

Finally, I owe the biggest debt of gratitude to my wife Pamela. She was there with me from the beginning and always patient and understanding. This thesis comes out of her unselfish support and encouragement. I thank her dearly and owe her much. 



\section{Contents}

1 Introduction and Overview 17

1.1 Motivation . . . . . . . . . . . . . . . . . . . 17

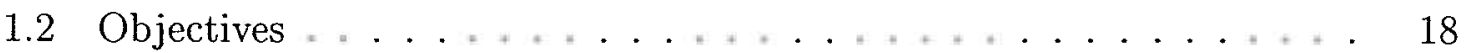

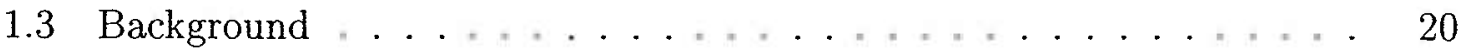

1.3.1 Iterative Methods . . . . . . . . . . . . . . 21

1.3.2 Exact Inverse Methods . . . . . . . . . . . . . . . . . . 24

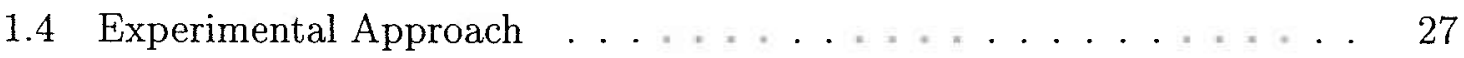

1.4.1 Numerical Results . . . . . . . . . . . . . . . . 27

1.4 .2 Modal Mapping Experiments . . . . . . . . . . . . 31

1.5 Outline . . . . . . . . . . . . . . . . . . . . 32

2 Review of Sound Propagation in a Shallow-Water Waveguide 34

2.1 Forward Problem ... . . . . . . . . . . . . . . . . . . 34

2.1.1 Point-source spectrum and plane-wave reflection coefficient . $\quad 35$

2.1.2 Cylindrical coordinates: The Hankel Transform $\ldots \ldots$. . . . 39

2.1.3 Normal Mode Representation . . . . . . . . . . . . . 42

2.1.4 Range-Dependent Modal Representation .......... 44

2.1.5 Effects Due to Source/Receiver Motion . . . . . . . . 45

2.2 Inverse Problem . . . . . . . . . . . . . . . . . . 48

$2.2 .1 \quad$ Perturbative Inversion $\ldots \ldots \ldots \ldots \ldots$

2.2 .2 Gelfand-Levitan Method . . . . . . . . . . . 52 
3 Wavenumber Estimation Techniques $\quad 56$

3.1 Introduction . . . . . . . . . . . . . . . . 56

3.2 High-resolution wavenumber estimation . . . . . . . . 60

3.2.1 Parametric Modeling Approach to Spectral Estimation . . . . 63

3.2 .2 Autoregressive Spectral Estimation . . . . . . . . . . 64

3.3 Performance of Modified Covariance AR frequency estimator . . . . 72

3.3.1 Frequency Estimation in the Presence of Noise . . . . . . . 72

3.3.2 Frequency Resolution . . . . . . . . . . . . . . . . 74

3.3.3 Size of Data Vector and Model Order Selection . . . . . 75

3.3.4 Application of AR estimator to acoustic data $\ldots \ldots \ldots 79$

3.4 Wavenumber Estimates for Range-Dependent Synthetic Acoustic Data 81

3.4.1 Inversion Techniques Workshop . . . . . . . . . . 82

3.4.2 Range-Dependent Sediment - TC3 _. . . . . . . . 89

3.5 Wavenumber estimation in random media - Internal Waves . . . . 96

4 Modal Mapping Experiment (MOMAX) 102

4.1 Introduction . . . . . . . . . . . . . . . . . . . 102

4.2 Experiment Overview . . . . . . . . . . . . . . . . . 103

4.2 .1 Data Reduction . . . . . . . . . . . . . . . . 107

4.3 MOMAX 97 and SWAT $01 \ldots \ldots \ldots \ldots \ldots$

4.3 .1 Environment . . . . . . . . . . . . . . . . 108

4.4 Summary of Measurements . . . . . . . . . . . . . . . . 110

$4.4 .1 \quad$ MOMAX $97 \ldots \ldots \ldots \ldots \ldots \ldots$

$4.4 .2 \quad$ SWAT . . . . . . . . . . . . . . . . . 113

5 Data Analysis $\quad 116$

5.1 Introduction . . . . . . . . . . . . . . . 116

5.2 Spatial measurements of complex-pressure fields . . . . . . . . 116

5.3 Wavenumber analysis of MOMAX data . . . . . . . . . . . . 119 
5.3 .1 Geometrical interpretation . . . . . . . . . . . . . 119

5.3.2 Modal Mapping - Single Mode . . . . . . . . . . . . . . . 122

5.4 Modal Mapping - Perturbative Inversion Results . . . . . . . . . 127

5.5 Modal Mapping - Moving Source Analysis . . . . . . . . . . 137

5.6 Gelfand-Levitan Results . . . . . . . . . . . . . . . . . . . 148

5.6.1 Effects of Density and Wavenumber Interval . . . . . . . . 149

5.6 .2 Shallow-water Exact Inversion . . . . . . . . . . . . 151

6 Summary and Conclusions $\quad 156$

6.1 Conclusions . . . . . . . . . . . . . . . . . . . 157

6.2 Suggestions for Future Work . . . . . . . . . . . . . 159 


\section{List of Figures}

1-1 Simple environmental model representing horizontally stratified waveguide . . . . . . . . . . . . . . . . . . . . 18

1-2 Three-dimensional environmental model. Sound speed profile is shown in upper left. Cross-range and down-range bathymetry profiles are shown in right upper plots. Full 3-D model is shown in bottom plot with source located at 30 meters depth in center of model. Sediment sound speed profile tracks the bathymetry. . . . . . . . . . 28

1-3 Plan view of $50 \mathrm{~Hz}$ Nx2D acoustic pressure field generated using KRAKEN for 3-D environmental model . . . . . . . . . . . . . . . 29

1-4 Plots of wavenumber evolution with range and azimuth for 3-D environment. Top plot is mode 1 and bottom is mode 2 for $50 \mathrm{~Hz}$ field. . 30

1-5 Model data comparison for MOMAX experiment at $75 \mathrm{~Hz} \ldots . .32$

2-1 Layered sediment model used for calculation of plane-wave reflection coefficient. . . . . . . . . . . . . . . . . . 38

2-2 Angle definitions for coordinate transforms in Cartesian and wavenumber space ... . . . . . . . . . . . . . . . . . . 40

2-3 Source/Receiver geometry considered by Hawker for moving source problem [38]. $\ldots \ldots \ldots \ldots \ldots$

2-4 Geometry used by Schmidt and Kuperman for analysis of Doppler shifts due to source/receiver motions [73] . . . . . . . . . . . 47 
2-5 1-Dimensional inverse scattering problem model. Inverse problem is to determine the potential $\mathrm{V}(\mathrm{z})$ of the scattering region.

3-1 $50 \mathrm{~Hz}$ synthetic pressure field data for waveguide with unknown rangedependent sediment properties. $1000 \mathrm{~m}$ and $2000 \mathrm{~m}$ data segments are shown as used for making wavenumber estimates.

3-2 Comparison of spectral estimates for $2000 \mathrm{~m}$ data aperture. Classical PSD result is solid, high-resolution result is dashed, and true wavenumber values are dotted.

3-3 Comparison of spectral estimates for $1000 \mathrm{~m}$ data aperture. Classical PSD result is solid, high-resolution result is dashed, and true wavenumber values are dotted. . . . . . . . . . . . . . . . .

3-4 Spectral estimates for 100 realizations of signal with 2 sinusoids in noise. $\mathrm{SNR}=20 \mathrm{~dB}, f_{1}=0.4 \mathrm{~Hz}, f_{2}=0.35 \mathrm{~Hz}$. AR model order is indicated at top of each plot. . . . . . . . . . . . .

3-5 Spectral estimates for 100 realizations of signal with 2 sinusoids in noise. $\mathrm{SNR}=20 \mathrm{~dB}, f_{1}=0.4 \mathrm{~Hz}, f_{2}=0.35 \mathrm{~Hz}$. AR model order is indicated at top of each plot. . . . . . . . . . 78

3-6 Environment model for Inversion Workshop TC0. . . . . . . . . . 83

3-7 Sliding Window spectral estimates for $50 \mathrm{~Hz}$ TC0. Triangles are the range-dependent horizontal wavenumbers determined using KRAKEN plotted over the wavenumber estimates. . . . . . . . . . . . . .

3-8 Wavenumber estimates for TC0 (dots) plotted with values for the starting range, center range, and end range of the transform aperture.

3-9 Shelfbreak environmental model for Inversion Workshop TC2. . . . . 85

3-10 Wavenumber estimates showing range-independent region after the shelfbreak for Inversion Workshop TC2.

3-11 Wavenumber estimates using adaptive AR model order selector showing wavenumber evolution for Inversion Workshop TC2. . . . . . . . 
3-12 Wavenumber estimates for order 66 AR estimator showing wavenumber evolution Inversion Workshop TC2 data with $60 \mathrm{~dB}$ noise added. . . 88

3-13 Environment model for Inversion Workshop TC3. . . . . . . . . . 89

3-14 Wavenumber estimates for range-dependent sediment of Inversion Workshop TC3. . . . . . . . . . . . . . . . 90

3-15 Wavenumber estimates for range-dependent sediment of Inversion Workshop TC3 with range-independent segments identified. . . . . . . 9 91

3-16 Spectral estimates for full apertures of range-independent regions identified for Inversion Workshop TC3. . . . . . . . . . . . . 92

3-17 Compressional wave speed in the sediments for Inversion Workshop TC3. Open circles are background profile used for perturbative inversion, solid line is ground truth, and dashed line is inferred profile.

3-18 Resolution Kernels for inversion results of Inversion Workshop TC3. Background represents regions 1 and 3 and Intrusion represents region 2. 93

3-19 Complex pressure field modeled using inferred sediment sound speed profile compared to inversion workshop TC3 data at $50 \mathrm{~Hz}$. . . . . 94

3-20 Complex pressure field modeled using inferred sediment sound speed profile compared to inversion workshop TC3 data at $100 \mathrm{~Hz} . \ldots . .95$

3-21 Range-dependent sound velocity field for IW wavenumber estimates [76]. Background profile is on left. . . . . . . . . . . . 98

3-22 $100 \mathrm{~Hz}$ TL plots for internal wave study. $5 \mathrm{~km}$ sub-plots show departure of internal wave measurements from unperturbed case. . . . . . . 99

3-23 Sliding window wavenumber estimates with KRAKEN results (dashed) plotted for unperturbed case. . . . . . . . . . . . . 100

3-24 Sliding window wavenumber estimates with KRAKEN results (dashed) plotted for IW case. . . . . . . . . . . . . . . . 100

4-1 Sketch representation of MOMAX experimental layout . . . . . 103

4-2 MOMAX drifter buoy components. . . . . . . . . . . . 105 
4-3 Hydrophone depth record for typical MOMAX experimental run. . . 106

4-4 MOMAX Experiment Site . . . . . . . . . . . . . . . . . 109

$4-5$ (a) SSP for all experiments (b) Mean and $+/-1$ std $\ldots \ldots . . . .112$

4-6 Sound speed variability during SWAT experiment 1 determined from temperature variability recorded on NRL T-string. . . . . . . . . . 114

5-1 $50 \mathrm{~Hz}$ spatial pressure field measured with source located at the origin of the coordinate system. Top plot is pressure magnitude, center plot is phase, and bottom plot is drifter track geometry. . . . . . . . 117

$5-2 \quad 50 \mathrm{~Hz}$ data plotted as a function of time with corresponding separation distance between source and receiver. Initial range for bottom plot determined from a single GPS measurement. . . . . . . . . . . 119

5-3 Source/receiver separation speeds determined from GPS and phase measurements of $50 \mathrm{~Hz}$ complex-pressure field. . . . . . . . 120

5-4 Schematic representing mapping of spatial pressure data to a range grid.121

5-5 Source/Receiver geometry for SWAT $20 \mathrm{~Hz}$ experiment. . . . . . . . 123

$5-620 \mathrm{~Hz}$ Complex pressure measured on Larry. . . . . . . . . . . 125

5-7 Modal evolution for single mode at $20 \mathrm{~Hz}$ on Larry. . . . . . . . . 125

$5-820 \mathrm{~Hz}$ Complex pressure measured on Moe. . . . . . . . . . . . . 126

$5-9$ Modal evolution for single mode at $20 \mathrm{~Hz}$ on Moe. . . . . . . . 126

5-10 MOMAX I experiment 4 geometry and bathymetry. Depth is indicated by the colorbar on the right in meters. For this experiment, the buoy moved very little as indicated by the track at the upper left, and the source was moving slowly along a constant bearing. . . . . . . 127

5-11 $75 \mathrm{~Hz}$ pressure field magnitude for MOMAX 97 experiment 4 plotted over the drift track. Drift track is plotted relative to source position. 128

5-12 $75 \mathrm{~Hz}$ complex pressure plotted as a function of range for MOMAX 97

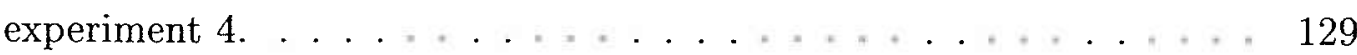

5-13 Horizontal Wavenumber Spectrum for full aperture $75 \mathrm{~Hz}$ data. . . 129 
5-14 Starting profile and inferred profile for MOMAX 97 experiment 4. Density was $1.8 \mathrm{~g} / \mathrm{cm}^{3}$ and no attenuation was used. . . . . . . 130

5-15 Comparison of $75 \mathrm{~Hz}$ measured and synthetic pressure magnitude data for inversion of MOMAX 97 experiment $4 \ldots \ldots$. . . . . . . 131

5-16 MOMAX 97 experiment 2 geometry and bathymetry. Buoy track is shown in upper left and source track in bottom right. Water depth is indicated by the colorbar on the right in meters. . . . . . . . . 132

5-17 $75 \mathrm{~Hz}$ pressure field magnitude for MOMAX 97 experiment 2 plotted over the drift track. Drift track is plotted relative to source position.

5-18 Horizontal wavenumber spectrum estimate for MOMAX 97 experiment 2 data at $75 \mathrm{~Hz}$. AR estimate is solid line, PSD estimate is dashed.

5-19 Starting profile (thin) and inferred (thick) sediment sound speed profile for MOMAX 97 experiment 2. Density was $1.8 \mathrm{~g} / \mathrm{cm}^{3}$ with no attenuation used.

5-20 Comparison of measured and synthetic data for inversion of MOMAX 97 experiment 2.

5-21 Source receiver geometry for SWAT experiment 2 with region of source track doubling back on itself. Segment 2 indicates time when source is moving away from buoy and segment 3 indicated time when source is closing on buoy. . . . . . . . . . . . . . .

$5-2250 \mathrm{~Hz}$ pressure field for SWAT experiment 2. Source is moving at a constant speed of $2 \mathrm{~m} / \mathrm{s} \ldots \ldots \ldots \ldots \ldots \ldots \ldots$

5-23 Sound speed variability measured on NRL T-strings during acoustic measurements along segments 2 and 3 . Colorbars indicate sound velocity in $\mathrm{m} / \mathrm{s}$.

$5-2450 \mathrm{~Hz}$ pressure magnitude data for overlapping tracks of segments 2 and $3 \ldots \ldots \ldots \ldots \ldots \ldots \ldots \ldots \ldots$

5-25 Modal evolution for $50 \mathrm{~Hz}$ data of segment $2 \ldots \ldots \ldots \ldots$ 
5-26 Waterfall plot of spectrum amplitudes with range for $50 \mathrm{~Hz}$ data of

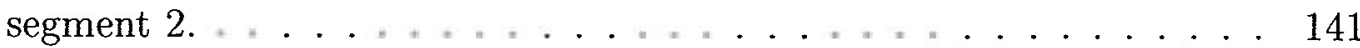

5-27 Modal evolution for $50 \mathrm{~Hz}$ data of segment $3 \ldots \ldots \ldots 141$

5-28 Waterfall plot of spectrum amplitudes with range for $50 \mathrm{~Hz}$ data of segment 3. . . . . . . . . . . . . . . . . . . . . . 142

5-29 Evolution of modes 1 and 2 with range showing bias due to source motion over segments 2 and $3 \ldots \ldots \ldots \ldots \ldots$

5-30 Doppler corrected wavenumber estimates for modes 1 and 2 over data

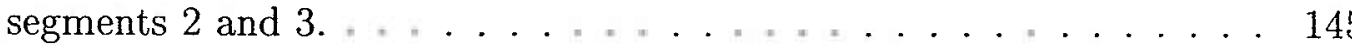

5-31 $50 \mathrm{~Hz}$ synthetic pressure fields generated for moving source analysis. SNR is $40 \mathrm{~dB}$. Source speeds were $10 \mathrm{~m} / \mathrm{s} \ldots \ldots \ldots \ldots . \ldots 147$

5-32 Spectral estimates for moving source data with stationary values of horizontal wavenumbers indicated by dashed line. Source speed was

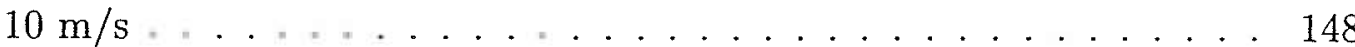

5-33 Reflection Coefficient at $20 \mathrm{~Hz}$ with and without density discontinuity at water-bottom interface.

5-34 Fourier transform of reflection coefficient with and without density discontinuity.

5-35 Comparison of inferred sound speed profile in sediment using the GelfandLevitan method. Results are shown with and without density discontinuity and for truncated wavenumber integrals.

5-36 Pekeris environment used to calculate shallow water Green's function. 153

5-37 Exact Green's function for Pekeris problem compared to OASES and KRAKEN. . . . . . . . . . . . . . . . . . . . 154

5-38 Reflection coefficient determined from shallow-water Green's function. 154

5-39 Fourier transform of Pekeris reflection coefficient with effect of density discontinuity removed. . . . . . . . . . . . . . . 155 
5-40 Sediment sound speed profile inferred using the Gelfand-Levitan method for the Pekeris problem in figure $5-36 \ldots \ldots \ldots \ldots \ldots$ 


\section{List of Tables}

3.1 SNR (dB) vs $10 \log _{10}$ (mse) for estimation of frequency $=2.5 / 2 \pi \mathrm{Hz}$. .

3.2 SNR (dB) vs bias of estimates for frequencies $f_{1}=2.5 / 2 \pi \mathrm{Hz}$ and $f_{3}=2.3 / 2 \pi \mathrm{Hz} \ldots \ldots \ldots \ldots \ldots \ldots \ldots \ldots \ldots \ldots \ldots \ldots$

3.3 SNR (dB) vs $10 \log _{10}(m s e)$ for estimation of $f_{o}$ using ESPRIT and AR. $f_{o}=0.4 \mathrm{~Hz}, \delta f=0.011 \mathrm{~Hz}$.

3.4 Probability of success for estimation of $\mathrm{f}=0.4 \mathrm{~Hz}$ with $\mathrm{SNR}=10 \mathrm{~dB}$, model order $\mathrm{p}=20$, and increasing data length. Percent correct for estimating $(\mathrm{f} 1 \pm 0.001) \mathrm{Hz} \ldots \ldots \ldots \ldots \ldots$

3.5 Probability of success for estimation of $\mathrm{f}=0.4 \mathrm{~Hz}$ with $\mathrm{SNR}=10 \mathrm{~dB}$ and increasing model order. . . . . . . . . . . . . . . . .

3.6 Pekeris ocean model. Frequency $=100 \mathrm{~Hz}$, source depth $=20.5 \mathrm{~m}$, and receiver depth $=15.5 \mathrm{~m}$.

3.7 Modal eigenvalues and corresponding amplitudes at source and receiver for Pekeris ocean model. . . . . . . . . . . . . . . . . . . . 80

3.8 SNR ( $\mathrm{dB})$ vs $10 \log _{10}$ (mse) of eigenvalues for different data aperture lengths.

4.1 Overview of source and buoy deployments for MOMAX 97. . . . . 111

4.2 Overview of source and buoy deployments for SWAT . . . . . . . 113

5.1 Pekeris waveguide model parameters for moving source experiment. . 145

5.2 Modal eigenvalues and amplitudes for $50 \mathrm{~Hz}$ Pekeris. . . . . . . 146 
5.3 Wavenumber and group velocities estimated from moving source spectra compared to true values. Source speed was $10 \mathrm{~m} / \mathrm{s} \ldots \ldots \ldots 146$ 


\section{Chapter 1}

\section{Introduction and Overview}

\subsection{Motivation}

Any review of the underwater acoustic or ocean science literature will immediately impress upon the reader the importance of acoustics as a tool for understanding not only acoustic problems such as propagation and scattering, but many other ocean science problems as well, including measurements of ocean temperature and currents, monitoring of marine mammal activity, and characterization of different fish species, to name a few. Whatever the application, each benefits from increased knowledge of the propagation environment. Consistent with this, in a much referenced paper by Hamilton[36], a case was made for the importance of geoacoustic modeling of the seabed. Hamilton asserts that knowledge of geoacoustic models is basic to the understanding of underwater acoustics. His statement of twenty years ago is particularly true now with today's requirement of rapid assessment where complex models based on realistic input parameters are used for prediction purposes. In shallow water environments, where the sound field is constantly interacting with the seafloor, knowledge of bottom geoacoustic properties is critical to making accurate predictions. It is this scenario that calls for a robust means of determining the geoacoustic properties of the seafloor. 


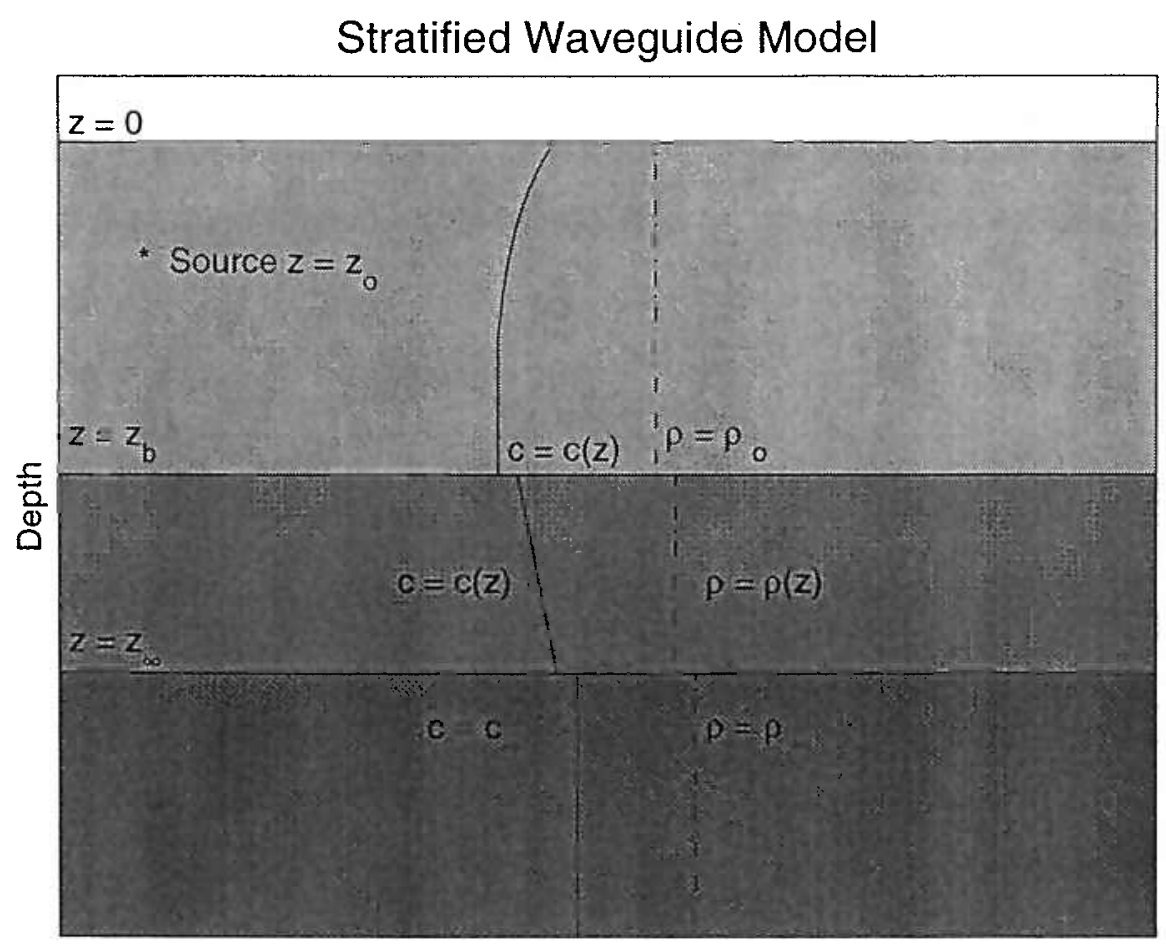

Figure 1-1: Simple environmental model representing horizontally stratified waveguide

\subsection{Objectives}

The objective of this thesis work is to investigate methods for inferring the properties of the seafloor from spectral measurements of point-source acoustic pressure field data in shallow-water environments. The data consist of complex pressure measured on synthetic aperture horizontal planar arrays for a point source at constant depth emitting several discrete tones [31]. A canonical model is represented in Figure 1-1. In this context, the spectral representation of the field is given by the depth-dependent Green's function versus horizontal wavenumber. As will be shown, the basis for the geoacoustic inverse problem is the dependence of the Green's function on the local properties of the waveguide. Theory and experiment have confirmed this relationship through application of Hankel transform-based methods for extracting the wavenum- 
ber spectrum for data measured on synthetic aperture line arrays [26] [55]. The work described in this thesis differs from previous work in that synthetic apertures are formed along arbitrary horizontal paths between source and receiver positions as opposed to being formed along radials at constant bearing. These measurements are unique in that they use precision Global Positioning System (GPS) measurements of source and receiver buoy positions to create the synthetic aperture used for processing. Examination of the point source spectra determined for sub-apertures of these horizontal arrays yields information about spatial variability in the seafloor. Emphasis is placed on extracting modal content and interpreting modal evolution in the context of the measurement geometry. Where appropriate, this includes Doppler effects due to source/receiver motion on the measured spectra. In this thesis, a procedure for the direct measurement of modal group velocity is presented through measurement of an observed Doppler shift. Using this result, the effect of Doppler can be removed from the wavenumber estimates. Along these same lines, another objective of this work is to achieve maximum resolution of discrete wavenumbers given short aperture field data to obtain high resolution geospatial maps of wavenumber content. In this effort, the use of high resolution spectral estimation techniques are examined as well as the effect of sound speed variations in the water column on extraction of closely spaced spectral values.

Given estimates of the point source spectrum, the next objective is to use the spectral data as input for the geoacoustic inverse problem. The experiments for this work were designed primarily as a means to provide input data to an inverse method based on making linear perturbations to a background starting model. This approach uses the discrete portion of the point source spectrum given by peaks in the Green's functions as input data to the inverse problem. Emphasis is placed on examining the applicability of the measurement data obtained on the arbitrary horizontal arrays to the perturbative inverse approach. During the course of this work, it was suggested that point-source spectral measurements as described might also be 
used as input data to one of the exact methods for geoacoustic inversion that does not require perturbations or iterations. Thus, a second inversion approach studied is an adaptation of the Gelfand-Levitan method for geoacoustic inversion using the point-source spectral data for shallow water. In this approach both the continuous and discrete portion of the Green's function are used to obtain the input data for the inverse algorithm. It will be shown that from these measurements, it may be possible to estimate the reflection coefficient at the water-bottom interface. The input for the inversion scheme is then the Fourier transform of the plane-wave reflection coefficient. Numerical results will be presented for this case with recommendations made for future experiments.

Clearly, by combining the spectral estimation efforts and the inversion efforts, the local nature of the Green's function can be exploited to obtain fully three-dimensional geoacoustic inversions.

\subsection{Background}

The history of seafloor characterization in the underwater acoustics community is a long one. Many different measurement and analysis techniques have been developed through the years to estimate bathymetry, seafloor roughness, and sediment composition. In probing the seafloor for its geoacoustic properties, several major classes of approaches have emerged. Frisk [27] breaks these classes into four categories that include inversion for bottom properties based on specific features, iteration of forward models, perturbative inversion, or exact inverse methods. Included in the class of iterating forward models are optimization methods such as simulated annealing or genetic algorithms. For the measurements and data to be discussed in regard to this thesis work, any of the latter three inversion method classes could be appropriate. However, of particular interest are the perturbative inverse approach and exact methods. It should be noted that there is a bit of overlap in these classifications in that 
both the perturbative inverse method and the iteration of forward models involve iteratively making perturbations to a starting model based on a priori information about the environment combined with measured field data. The differences arise from the nature of the perturbations, as well as the cost function defined to evaluate the results. These differences lead to a substantial difference in the number of iterations required and are discussed further in the following section where both approaches are categorically referred to as iterative methods. For any of these iterative methods, results from an inversion are compared to measured data and the model is perturbed again using the comparison information. Iteration continues until the model/data comparison falls within an acceptable range of the observed values. Exact methods differ from optimization methods in that they do not require a background model for determination of the model parameters. These methods are exact in the sense that they have either analytic or numerical forms of solution which make direct use of the field data in the reconstruction of the geoacoustic properties. Consequently, these methods do not suffer from any reliance on the approximations made for the forward-problem model, or the sometimes non-unique nature of the perturbed background model solutions. Following is a brief summary of previous work done using the two types of methods.

\subsubsection{Iterative Methods}

Much of the recent geoacoustic inversion literature has focused on algorithms which seek to determine a set of model parameters which minimize the mismatch between measured and simulated field data. This problem becomes increasingly difficult and computationally intensive as the number of model parameters to be determined is increased. Further complicating the problem is the presence of local minima inherent in the inverse problem. Rajan et al. [62][66] employed several different iterative approaches to perturb a starting model using measurements of the complex pressure field as input data. Using short aperture Hankel transforms to obtain eigenvalue 
information, Frisk et al. [26] used a perturbative-inverse algorithm to obtain compressional wave speed profiles for two adjacent, but geo-acoustically distinct, regions in Nantucket Sound. Ohta and Frisk[54][55] used a short-window sliding transform and iteration of forward models to further extend these methods to the range-dependent case. The perturbative inversion methods used by these authors were based on linear or quasi-linear inverse algorithms. To ensure linearity, the parameter search space was limited to small perturbations around the background model. Further, the operator on the parameter that gives an estimate of the data is assumed to be either monotonic, or Frechet differentiable, a condition that allows the linearization of a nonlinear function [64]. These requirements ensure that the inversion algorithm travels downhill and will arrive at the global minimum for the cost function as determined by linearizing the problem around the assumed background model. The benefits of the perturbative inverse method include the following: the search parameter space derived from the data limits the results to realistic values for the seafloor; the linear inverse equations allows for the determination of the variance and resolution of the parameter estimates; and the results do not depend on and do not require multiple full forward modeling runs. A limitation of these methods are their sensitivity to the starting model and the potential for arriving at at a non-unique solution due to minimizing the cost function based on an incorrect starting background model. To overcome these shortcomings, other nonlinear approaches have been investigated which allow a more thorough search of the model parameter space by employing random processes to perturb the starting model. The random nature of the perturbation makes them less sensitive to the starting model and avoids trapping in the local minima along with removing the monotonic requirement on the data. However, because of their random nature, global methods tend to converge more slowly and are more computationally expensive. Further, because global methods require repeated application of forward modeling runs, they are dependent on the forward model used. Two of the more popular global methods that have been used successfully in geoacoustic 
inversions are simulated annealing (SA) and genetic algorithms (GA). SA is based on the thermodynamic annealing principles governing crystal growth and seeks a minimum in parameter space through random perturbations of the model with acceptance of uphill steps probabilistically decreasing as time increases. Kuperman et al. [45] devised a fast SA algorithm to invert for wave-speeds and attenuation for slightly range-dependent environments. Collins et al. [15] used simulated annealing based on a full-field parabolic equation model to invert for range- and depth-dependent bottom geoacoustic parameters. GA are analogous to genetic evolution where perturbations to a model population are related to genetic crossover, or recombination, and mutations resulting in a new model of a given fitness. Models with high fitness, indicating a low mismatch between parent and offspring, are kept, while low fitness models are rejected. Different probabilistic models can be introduced into the algorithm to further refine the fitness criterion for the selection of parents in the formation of new offspring. GA was first used on synthetic data by Gerstoft[35], and then used on real broadband acoustic data by Hermand and Gerstoft [39] to determine bottom sediment properties and layer thicknesses. Combining the quick convergence properties of the local approaches with the full parameter space searches of global methods, Fallat and Dosso[23] have devised a hybrid approach based on downhill simplex and simulated annealing to invert for geoacoustic properties. Also seeking to minimize the mismatch between measured and simulated data, their method was shown to converge demonstrably faster than either a local or global method alone for a test case where the geoacoustic parameters were known.

Each of the inversion methods described above has its advantages and disadvantages. The computational expense of the various methods will be addressed in a forthcoming report from a recent workshop on geoacoustic inversion techniques. The report should provide a good summary of the computational requirements and effciency of various inverse methods [13]. Putting the other differences aside, the work is this thesis was motivated by experimental work designed to provide input data for 
a perturbative inverse method. Therefore, much of the development in this thesis is placed in the context of perturbative inversion.

\subsubsection{Exact Inverse Methods}

Much of the exact inverse work in determining geoacoustic properties considered for this thesis is found in the geophysical literature and is summarized by Merab[50]. The geophysical problem of interest is largely based on physics and mathematics in the form of the solution to inverse problems of quantum scattering theory. The connection between scattering theory and the acoustic problem is made by showing the geoacoustic inverse problem to be equivalent to determining the potential in one-dimensional inverse scattering problems for the Schrödinger equation. Of particular relevance to the solution of this problem are the methods of Gelfand and Levitan[34], and the trace methods formulated by Deift and Trubowitz[17] (DT). The formulation of these two solution alternatives begin with equivalent equations, boundary conditions, and assumptions.

The major difference between the two methods of solution is in the choice of input data for the inversion. The DT trace method uses the plane-wave reflection coefficient as a function of wavenumber as input data for the simultaneous solution of a coupled pair of equations. The solution is given in terms of a potential $q(z)$ that is directly related to the reflection coefficient. It will be shown in chapter 2 that the plane-wave reflection coefficient is directly related to the sediment properties thus establishing the relationship between the potential and the sound speed profile in the bottom. In its most general form, the solution proceeds with two unknown functions including the potential, $q(z)$, and $f(z, k)$ which is a solution to a one-dimensional Schrödinger equation of the form

$$
f^{\prime \prime}+\left(k^{2}-q\right) f=0
$$

The above equation is written and solved in terms of the function $f$ alone by using 
the following "trace formula" to eliminate $q(z)$ in equation (1.1),

$$
q(z)=\frac{2 i}{\pi} \int_{-\infty}^{\infty} k R(k) f^{2}(z, k) d k
$$

where the reflection coefficient, $R(k)$, is the input data and $k$ is the wavenumber.

The trace method was first applied to the shallow-water ocean acoustic problem by Stickler and Deift[77]. They showed how to recover the sound speed in a stratified ocean where the frequency was chosen so that no proper modes were excited. As input data to the inversion they required measurements of the normal derivative of a harmonic point source pressure field at the pressure-release surface. Stickler[78] removed the requirement of measuring the normal derivative of pressure, while keeping the restriction of no trapped modes, to recover the sound speed and density profiles in the sediment using trace methods. He used measurements of the sound field versus range at fixed depth for two distinct frequencies as input data.

In contrast to the trace methods which use the reflection coefficient directly, the Gelfand-Levitan (G-L) approach uses the Fourier transform of the reflection coefficient as input data to a Fredholm integral equation of the second kind. Merab[50] studied several different solution methods to the G-L integral equation for a deep water experiment. The reflection coefficient is obtained by measuring both the direct and bottom reflected field for a single-frequency point source in deep water and applying the Hankel Transform inversion technique described in [25]. The Fourier transform of the measured reflection coefficient is then used as the input for the following G-L type integral equation:

$$
K(z, y)+\hat{R}(z+y)+\int_{-\infty}^{z} \hat{R}\left(z^{\prime}+y\right) K\left(z, z^{\prime}\right) d z^{\prime}=0, \quad y<z
$$

where $\hat{R}(z)$ is the Fourier transform of the reflection coefficient $R(k)$, and the kernel, $K(z, z)$, is sought. Given $K(z, z)$, there exists a simple relation to find the desired 
potential $q(z)$.

$$
q(z)=2 \frac{d K(z, z)}{d z} .
$$

Merab illustrates the application of this approach using synthetic data for a known geoacoustic environment, taking care to avoid having trapped modes in the sediment.

Each of the above methods allows for the presence of bound states or trapped modes in the solution of the inverse problem. However, in each of the cited examples, cases were studied where bound states were not present. This was done because the bound states further complicate the mathematics and also require additional measurements to be made. Further, in the shallow-water environment of interest, it is often very difficult to measure the plane-wave reflection coefficient due to the presence of multiple reflections between the surface and the bottom. It is believed that this problem is compounded by the difficulty in making accurate measures of the phase of the complex pressure experimentally [64]. In the Modal Mapping Experiments (MOMAX) described in chapter 4 , it will be demonstrated that much progress has been made in this regard and the determination of the reflection coefficient for real data will be revisited. Recently, McLaughlin and Wang [51] have suggested a way of applying trace methods to obtain bottom sediment properties from shallow water data. In this formulation, trapped modes propagating in the water column are treated as bound states in the inverse problem and the potential includes the sound speed in the water column. McLaughlin and Wang use the depth-dependent Green's function obtained by Hankel transforming measurements of the complex-pressure field as their starting point. A straightforward relation between the Green's function and the reflection coefficient is applied to obtain the input data for the inversion. To account for the discrete spectrum present in the Green's function as it applies to the trace formula, McLaughlin applies a Darboux transform [17] to systematically remove the bound states from the problem. The result of each transform step yields a modified eigenvalue equation with one less bound state. All other bound states remain unchanged, and a new potential results. The Darboux transform is applied iteratively 
for each of the bound states and information is stored at each step. After removing all the bound states from the original eigenvalue problem, the inversion proceeds as if no bound states were present. A modified reflection coefficient is found for the now continuous spectrum and used as input data to the trace formulas to solve for an intermediate potential. The full potential is obtained from the intermediate result by reversing the Darboux transformation and adding the bound state information back in. Although not considered further in this thesis, the Darboux transform appears of interest for removing the effects of trapped modes in the sediments avoided by Merab. Further discussion of the Darboux transform for potential application to this problem can be found if Deift and Trubowitz [17] as well as Beals et al. [6].

\subsection{Experimental Approach}

In the next section, the concept of wavenumber evolution is illustrated using a numerical example. Results show the dependence of horizontal wavenumber on local waveguide properties. Following the numerical results, the experimental method for extracting local wavenumber content is briefly introduced.

\subsubsection{Numerical Results}

As stated, the input data for both the perturbative inverse method and GelfandLevitan method is the spectral data for the point source pressure field. The underlying premise is to measure the spatial variability of the wavenumber spectrum for a point source in a stratified medium to recover the geoacoustic properties of the seabed in three dimensions. As part of this work, both at-sea and numerical experiments have been conducted to test these concepts. Much of the work was driven by the notion that perturbative inversion methods would be used to infer sediment properties using eigenvalue information only. Thus, methods were investigated for extracting and mapping the wavenumber spectrum of the normal mode field for com- 

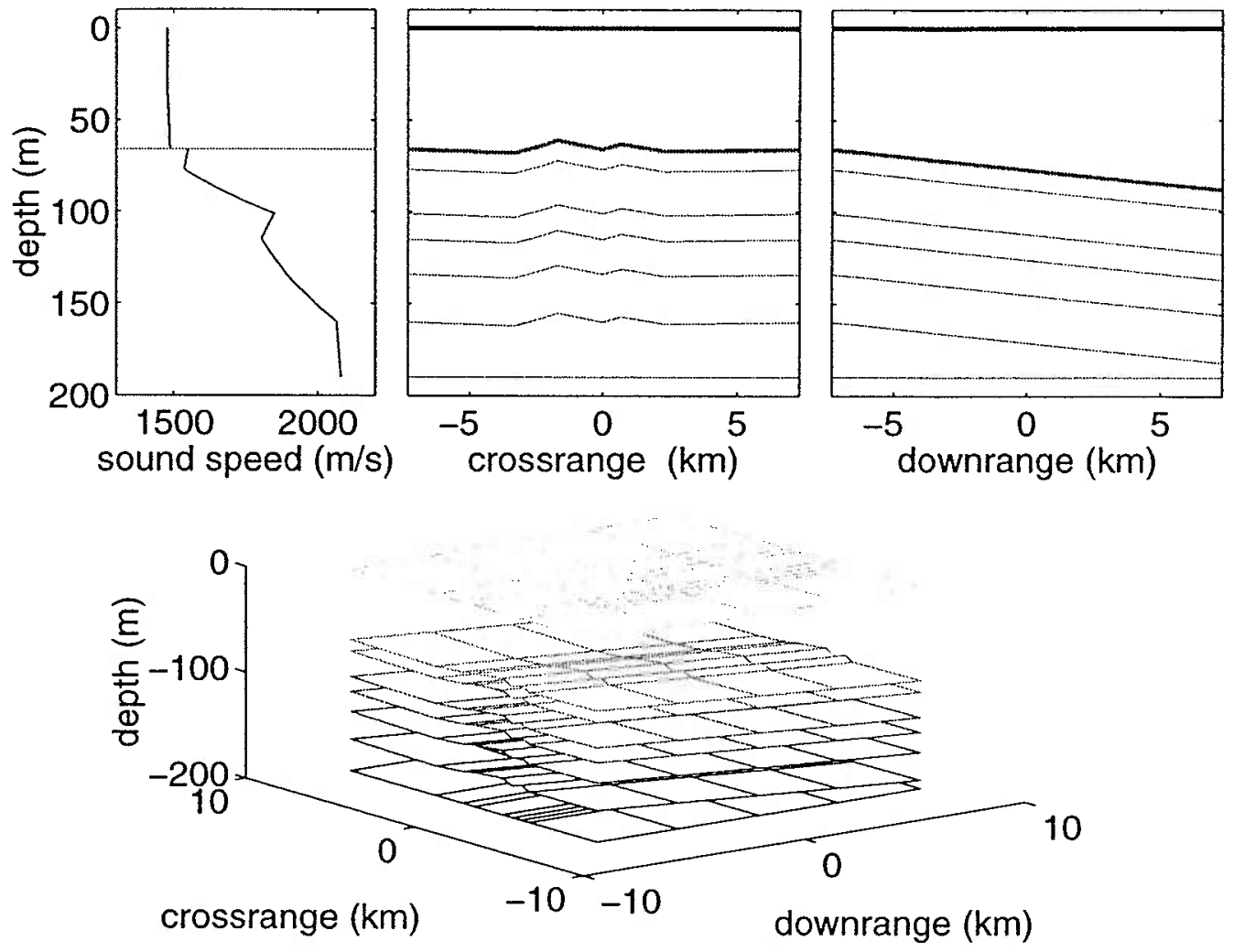

Figure 1-2: Three-dimensional environmental model. Sound speed profile is shown in upper left. Cross-range and down-range bathymetry profiles are shown in right upper plots. Full 3-D model is shown in bottom plot with source located at 30 meters depth in center of model. Sediment sound speed profile tracks the bathymetry.

plex shallow-water waveguide environments whose acoustic properties vary in three spatial dimensions[7]. For the waveguide depicted in figure 1-2, $50 \mathrm{~Hz}$ synthetic pressure data was generated using the KRAKEN normal mode program [59] as shown in figure 1-3. Short-aperture sliding window transforms were applied along radials to obtain estimates of the Green's function $g\left(k_{r}, r, \theta\right)$. Estimates where made using a classical spectral estimator based on the FFT with a Hanning window applied to the data for each aperture. Wavenumbers corresponding to the first two modes were extracted and plotted as a function of range and theta to create the modal maps in Figure 1-4. These maps represent the ideal case where modal information is obtained 


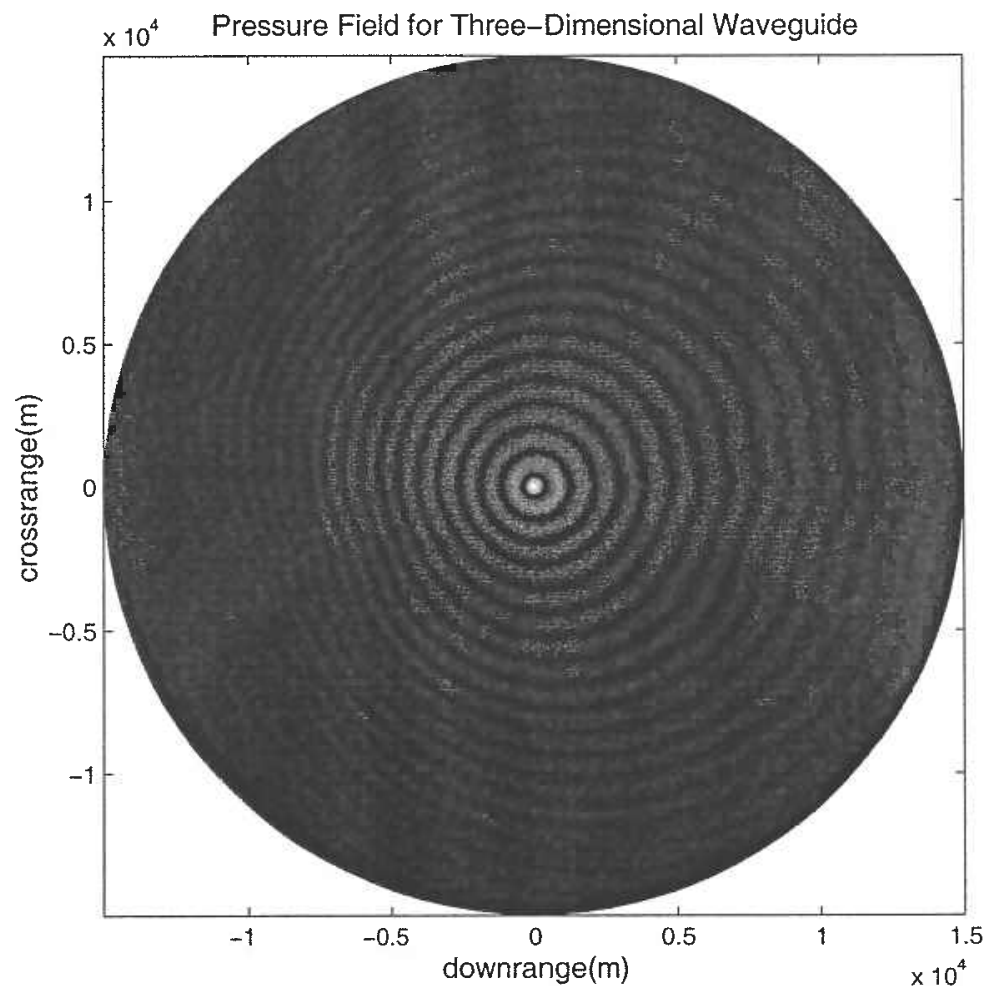

Figure 1-3: Plan view of $50 \mathrm{~Hz}$ Nx2D acoustic pressure field generated using KRAKEN for 3-D environmental model. 

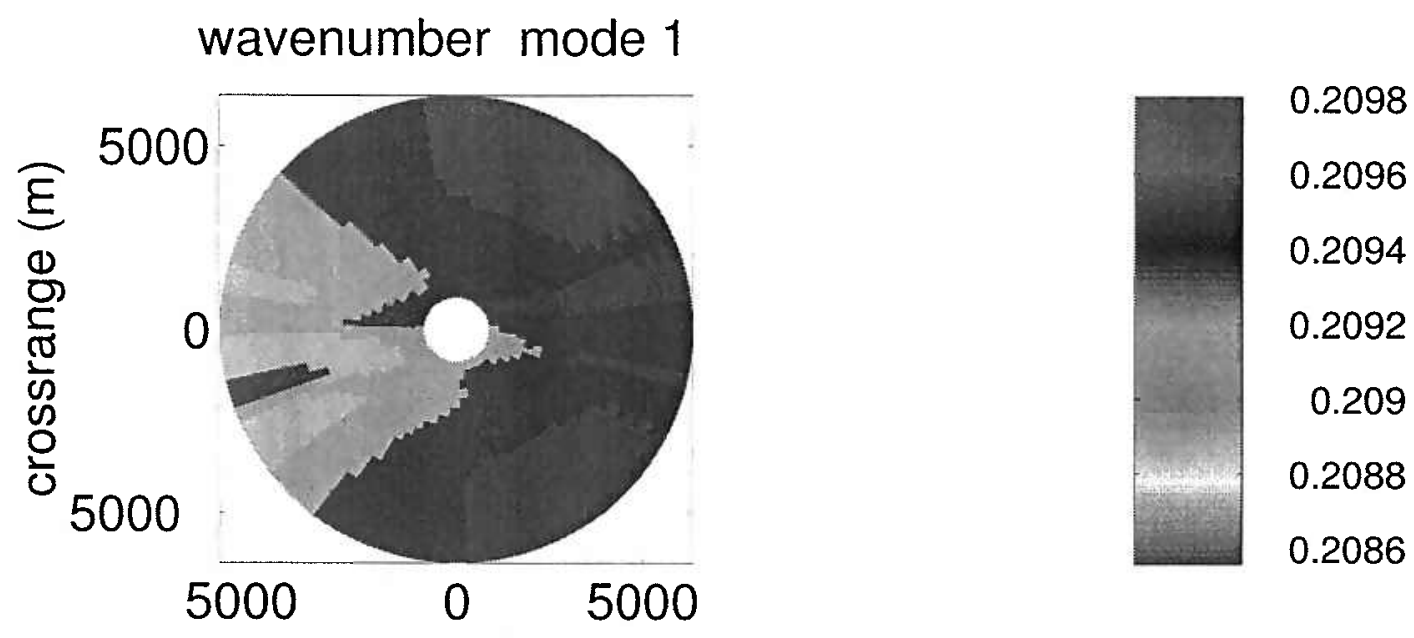

wavenumber mode 2
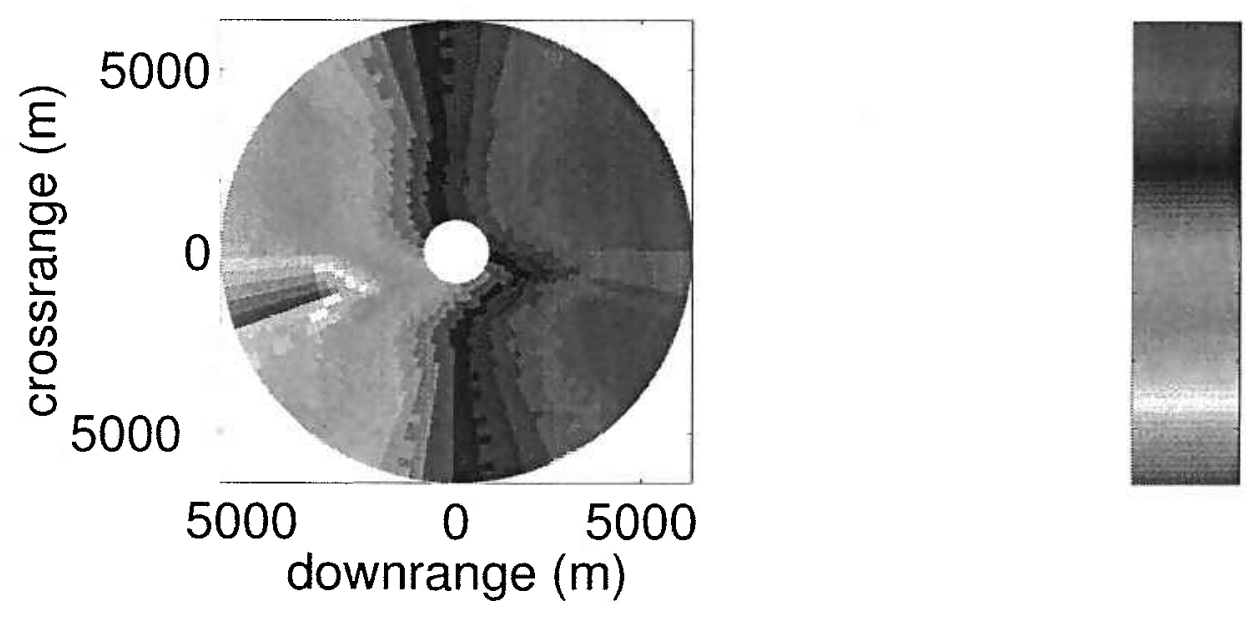

0.204

0.202

0.2

0.198

Figure 1-4: Plots of wavenumber evolution with range and azimuth for 3-D environment. Top plot is mode 1 and bottom is mode 2 for $50 \mathrm{~Hz}$ field. 
on a fully populated spatial array. The wavenumber estimates obtained represent the true wavenumber values well, but with low spatial resolution due to the length of the aperture used at each range step. To improve the spatial resolution of the spectral measurements, a portion of this thesis investigates a high-resolution spectral estimation technique to obtain modal wavenumber information using short aperture data [9]. This will be discussed in detail in chapter 3 .

\subsubsection{Modal Mapping Experiments}

To test these methods on real data, the Modal Mapping Experiments (MOMAX) were conducted in March 1997 and again in October 2000 off the New Jersey coast [29] along with MOMAX II held in February 1999 in the Gulf of Mexico [47]. In these experiments, drifting buoys equipped with a single hydrophone, GPS navigation, and radio telemetry were used to measure the acoustic field from a point source emitting several discrete tones in the frequency range $20-475 \mathrm{~Hz}$. High-resolution measurements of the sound field using several buoys were used to create two-dimensional synthetic aperture horizontal arrays. Modal maps were generated for both single receiver arrays, and for arrays formed by merging data from two calibrated receiving systems[8] [10]. Figure 1-5 shows one result from MOMAX comparing the measured field to a synthetic field generated using the recovered sound speed profile obtained using a perturbative inversion algorithm [62] as input data for an acoustic propagation model. These results illustrate the utility of the measured Green's function spectrum for characterizing the spatial variability of the acoustic waveguide and inferring its geoacoustic properties. Further, the phase of the measured acoustic data from MOMAX appears to be very robust and shows promise for extracting the reflection coefficient from the depth-dependent Green's function. For the case of no trapped modes in the sediment, these measurements may be used for inversion using the Gelfand-Levitan method. 


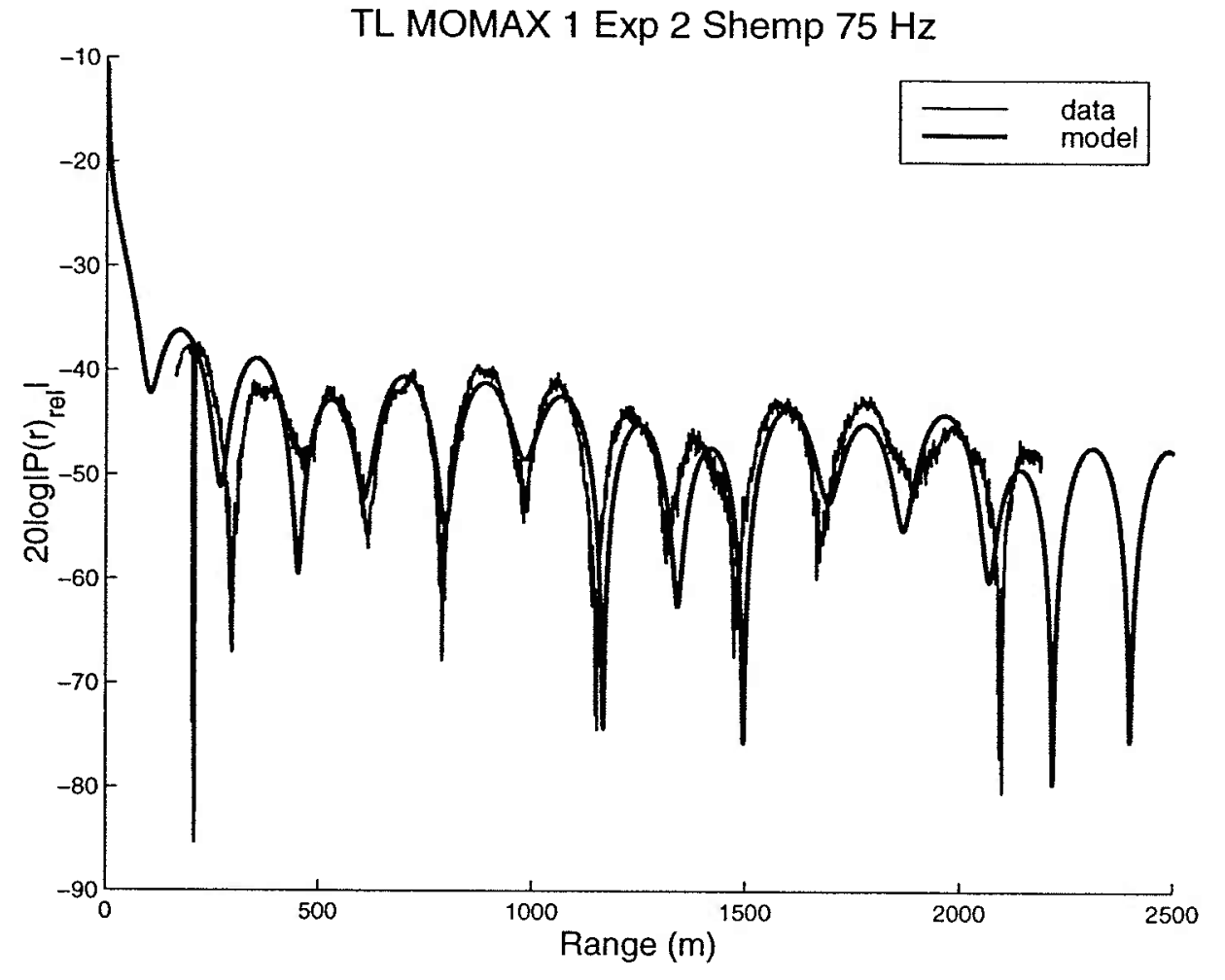

Figure 1-5: Model data comparison for MOMAX experiment at $75 \mathrm{~Hz}$

\subsection{Outline}

Chapter 2 presents an overview of the theory of wave propagation in a horizontally stratified shallow-water waveguide. The point-source solution is given in terms of the depth-dependent Green's function. The relationship between the Green's function and plane-wave reflection coefficient of the bottom is given highlighting the Green's functions dependence on bottom properties. Doppler effects on the Green's function due to source/receiver motions is also examined. The final part of the chapter describes the inverse problem. The perturbative inverse method is reviewed as applicable to the MOMAX experiments. Finally, the Gelfand- Levitan inverse method is reviewed with emphasis placed on applications using real data.

Chapter 3 presents data and results using synthetic data generated for rangedependent shallow-water environments. Data was provided as part of an ONR/SPAWAR sponsored inversion techniques workshop [40] that occurred in May, 2001. A high- 
resolution wavenumber estimator to extract range-dependent spectra was implemented for this work. In this chapter, the estimator will be described and its statistical characteristics examined for application to the MOMAX data. A related study will be described that examines the effect of sound speed perturbations in the water column on wavenumber measurements.

Chapter 4 describes in detail the modal mapping experiments. An overview of the experimental configuration is given along with a description of the data processing scheme. An overview of the operational environment for each experiment is then given along with supporting measurements of the water column and bathymetry. Finally, some example measurements and preliminary analysis of the MOMAX data are discussed.

Chapter 5 presents analysis of the MOMAX experimental data. Modal evolution is examined for the tracks created by the drifting buoys. Analysis is presented for the case of a single propagating mode where modal evolution can be determined analytically. For the case of a moving source, a method for measuring the group velocity is presented. MOMAX data are inverted using the perturbative method to obtain compressional wave speed profiles in the sediment. Finally, applicability of the MOMAX data to the Gelfand-Levitan inversion method is discussed with suggestions for further work.

The final chapter summarizes the present work with emphasis placed on new contributions. Suggestions for future efforts conclude the chapter. 


\section{Chapter 2}

\section{Review of Sound Propagation in a Shallow-Water Waveguide}

\subsection{Forward Problem}

The governing equations for sound propagation in shallow water are reviewed for the case of a horizontally stratified medium. The shallow water model, as shown in figure 1-1, is comprised of a stratified fluid layer, with sound velocity and density both functions of depth, overlying a seabed located at depth $z_{b}$ made up of multiple layers whose properties vary with depth.

The starting point is the full time-dependent acoustic wave equation in threedimensions for a time-harmonic source with radial frequency $\omega$, written [28],

$$
\rho_{o}(\mathbf{r}) \nabla \cdot\left[\frac{1}{\rho_{o}(\mathbf{r})} \nabla P(\mathbf{r}, t)\right]-\frac{1}{c^{2}(\mathbf{r})} \frac{\partial^{2} P(\mathbf{r}, t)}{\partial t^{2}}=-4 \pi \delta\left(\mathbf{r}-\mathbf{r}_{\mathrm{o}}\right) e^{-i \omega t}
$$

where $\mathbf{r}$ represents the spatial coordinates $(x, y, z), \mathbf{r}_{\mathbf{o}}$ is the source position, $t$ is time, $\delta(\mathbf{r})$ is the Dirac delta function, and $\nabla \equiv \frac{\partial}{\partial x} \hat{i}+\frac{\partial}{\partial y} \hat{j}+\frac{\partial}{\partial z} \hat{k}$. The density $\rho_{o}$ and sound speed $c$ are functions of position and suggest the influence of the propagation environment on the solution to the wave equation. Using the following definition for 
the Fourier transform in going from the time domain to the frequency domain,

$$
\mathcal{F} \cdot \mathcal{T} .\{x(t)\}=X(\omega)=\frac{1}{\sqrt{2 \pi}} \int_{-\infty}^{+\infty} x(t) e^{i \omega t} d t
$$

and applying it to (2.1), the time-independent Helmholtz equation results,

$$
\rho_{o}(\mathbf{r}) \nabla \cdot\left[\frac{1}{\rho_{o}(\mathbf{r})} \nabla p(\mathbf{r})\right]+k^{2}(\mathbf{r}) p(\mathbf{r})=-4 \pi \delta\left(\mathbf{r}-\mathbf{r}_{\mathbf{o}}\right)
$$

Here, angular frequency is defined by $\omega=2 \pi f$, for frequency $f$, and $k(\mathbf{r}) \equiv \omega / c(\mathbf{r})$ is the total acoustic wavenumber.

The wave equation can be simplified for our case by considering the solutions in regions of constant density. For a stratified waveguide where density is constant within each layer, equation (2.3) takes the form

$$
\left[\nabla^{2}+k^{2}(\mathbf{r})\right] p(\mathbf{r})=-4 \pi \delta(x) \delta(y) \delta\left(z-z_{o}\right)
$$

for the source located at the origin of the $(\mathrm{x}, \mathrm{y})$-plane and at a depth $z_{\boldsymbol{o}}$.

\subsubsection{Point-source spectrum and plane-wave reflection coef- ficient}

For a waveguide with properties that vary only as a function of depth, the solution to (2.4) proceeds by reducing the equation to one in the depth coordinate only. Appropriate boundary conditions are then applied at the air-sea and water-bottom interfaces. Defining the two-dimensional inverse Fourier transform operator in spatial coordinates by [28],

$$
\mathcal{I} . \mathcal{F} \cdot \mathcal{T} .\{f(x, y)\}=F\left(k_{x}, k_{y}\right)=\frac{1}{2 \pi} \int_{-\infty}^{+\infty} \int_{-\infty}^{+\infty} f(x, y) e^{-i\left(k_{x} x+k_{y} y\right)} d x d y
$$


and applying it to both sides of (2.4), the following equation results,

$$
\left[\frac{d^{2}}{d z^{2}}+k^{2}-k_{x}^{2}-k_{y}^{2}\right] g\left(k_{x}, k_{y} ; z, z_{o}\right)=-2 \delta\left(z-z_{o}\right)
$$

Here $k_{z}$ is the vertical wavenumber defined as $k_{z}=\sqrt{k^{2}-k_{x}^{2}-k_{y}^{2}}$, with $k_{x}$ and $k_{y}$ the horizontal wavenumbers with respect to a Cartesian coordinate system in wavenumber space. In the above, it is required that $\operatorname{Im}\left\{k_{z}\right\} \geq 0$ in order to ensure the field is finite as $z \rightarrow \infty$. The solution $g\left(k_{x}, k_{y} ; z, z_{o}\right)$ is the depth-dependent Green's function or point-source spectrum for the depth-separated wave equation. The Green's function is related to the acoustic pressure field through the conjugate Fourier transform pairs given by:

$$
\begin{aligned}
& p\left(x, y ; z, z_{o}\right)=\frac{1}{2 \pi} \int_{-\infty}^{\infty} \int_{-\infty}^{\infty} g\left(k_{x}, k_{y} ; z, z_{o}\right) e^{i\left(k_{x} x+k_{y} y\right)} d k_{x} d k_{y} \\
& g\left(k_{x}, k_{y} ; z, z_{o}\right)=\frac{1}{2 \pi} \int_{-\infty}^{\infty} \int_{-\infty}^{\infty} p\left(x, y ; z, z_{o}\right) e^{-i\left(k_{x} x+k_{y} y\right)} d x d y
\end{aligned}
$$

The solution to the depth-dependent equation (2.6) is obtained by incorporating into the total solution, the independent solutions $u_{T}\left(k_{x}, k_{y} ; z\right)$ and $u_{B}\left(k_{x}, k_{y} ; z\right)$ which satisfy the top and bottom boundary conditions, respectively. The general form of the depth-dependent Green's function satisfying both boundary conditions can be written,

$$
g\left(k_{x}, k_{y} ; z, z_{o}\right)=-\frac{u_{T}\left(k_{x}, k_{y} ; z_{<}\right) u_{B}\left(k_{x}, k_{y} ; z_{>}\right)}{W\left(z_{o}\right)} ; \quad 0 \leq z \leq z_{b}
$$

where $z_{<}=\min \left(z, z_{o}\right), z_{>}=\max \left(z, z_{o}\right)$ and $W\left(z_{o}\right)$ is the Wronskian, given in terms of the solutions $u_{T}$ and $u_{B}$ evaluated at the source location by,

$$
W\left(z_{o}\right)=u_{T}\left(z_{o}\right) u_{B}^{\prime}\left(z_{o}\right)-u_{T}^{\prime}\left(z_{o}\right) u_{B}\left(z_{o}\right)
$$

For an isovelocity water column, the solutions $u_{T}$ and $u_{B}$ can be expressed in 
terms of up-going and down-going plane waves along with their boundary interactions characterized by the plane-wave reflection coefficients $R_{T}\left(k_{r}\right)$ and $R_{B}\left(k_{r}\right)$ at the top and bottom interfaces. Expressing the reflection coefficients as a function of horizontal wavenumber, $k_{r}$, the two independent solutions are given by,

$$
\begin{gathered}
u_{T}\left(k_{r} ; z\right)=A\left[e^{-i k_{z} z}+R_{T}\left(k_{r}\right) e^{k_{z} z}\right], \\
u_{B}\left(k_{r} ; z\right)=B\left[e^{-i k_{z} z}-R_{B}\left(k_{r}\right) e^{k_{z} z}\right],
\end{gathered}
$$

for which the Wronskian becomes,

$$
W=2 i k_{z} A B\left(1-R_{T} R_{B} e^{2 i k_{z} z_{b}}\right),
$$

where $A$ and $B$ are arbitrary constants and $k_{r}=\sqrt{k_{x}^{2}+k_{y}^{2}}$. Substituting these expressions into (2.9), the depth-dependent Green's function for a shallow water waveguide is

$g\left(k_{r} ; z, z_{o}\right)=\frac{i\left\{e^{i k_{z}\left|z-z_{o}\right|}+R_{T}\left(k_{r}\right) e^{i k_{z}\left(z+z_{o}\right)}+R_{B}\left(k_{r}\right) e^{2 i k_{z} z_{b}}\left[e^{-i k_{z}\left(z+z_{o}\right)}+R_{T}\left(k_{r}\right) e^{-i k_{z}\left|z-z_{o}\right|}\right]\right\}}{k_{z}\left[1-R_{T}\left(k_{r}\right) R_{B}\left(k_{r}\right) e^{2 i k_{z} z_{b}}\right]}$.

This expression for the Green's function is a spectral representation of the propagating field in terms of horizontal wavenumber and shows explicitly its dependence on the boundary conditions of the waveguide. For the ocean acoustic problem, the air-sea interface is approximated by a pressure-release surface with boundary condition given by $R_{T}=-1$. The depth-dependent Green's function is then an explicit function of the plane-wave reflection coefficient at the water-bottom interface. For example, for a stratified seabed with multiple layers, as illustrated in figure $(2-1), R_{b}\left(k_{r}\right)$ can be written as a sum including the plane-wave reflection coefficients calculated between adjacent layers [44], and a phase term that depends on the layer depths. 


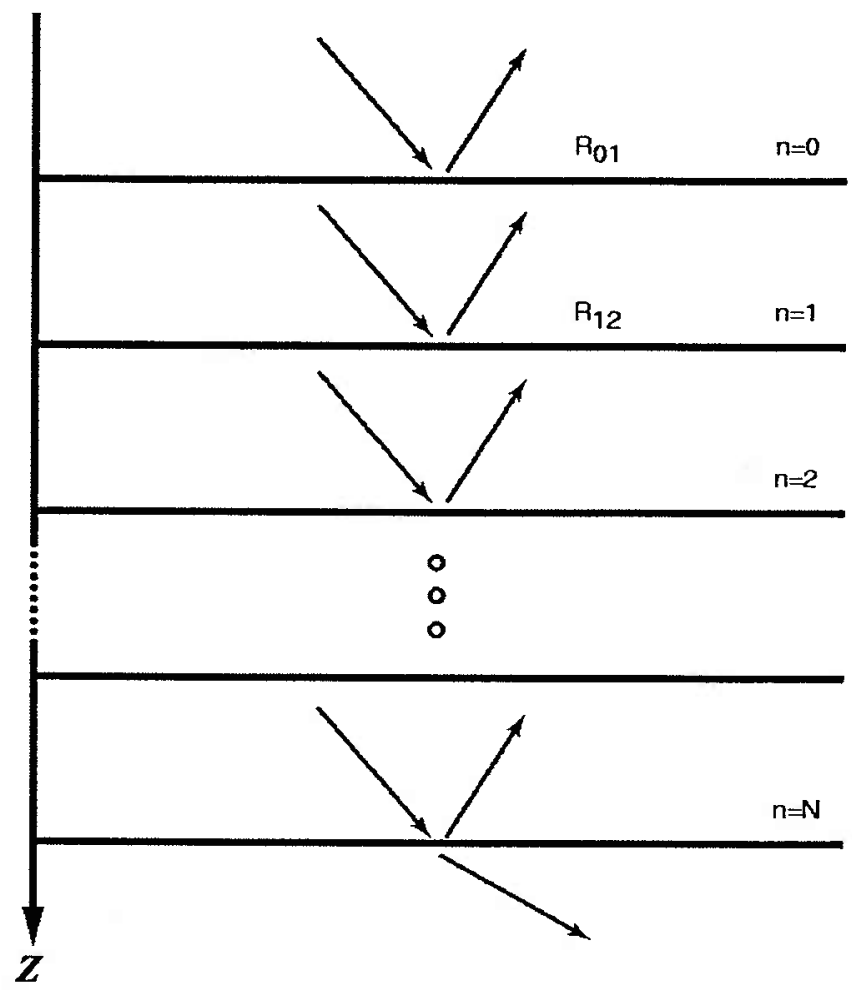

Figure 2-1: Layered sediment model used for calculation of plane-wave reflection coefficient.

$$
\begin{aligned}
& R_{b}\left(k_{r}\right)=\frac{1}{R_{01}} \quad+\frac{\left[1-\left(1 / R_{01}\right)^{2}\right] e^{-2 i k_{1 z} d_{1}}}{R_{01}^{-1} e^{-2 i k_{1 z} d_{1}}}+ \\
& \frac{1}{R_{12}}+\frac{\left[1-\left(1 / R_{12}\right)^{2}\right] e^{-2 i k_{22}\left(d_{2}-d_{1}\right)}}{R_{12}^{-1} e^{-2 i k_{2 z}\left(d_{2}-d_{1}\right)}}+\cdots \\
& \frac{1}{R_{(N-1) N}}+\frac{\left[1-\left(1 / R_{(N-1) N}\right)^{2}\right] e^{-2 i k_{n z}\left(d_{N}-d_{N-1}\right)}}{R_{(N-1) N}^{-1} e^{-2 i k_{n z}\left(d_{N}-d_{N-1}\right)}}+ \\
& R_{N(N+1)} \text {, }
\end{aligned}
$$

where $R_{i j}$ is the reflection coefficient between two adjacent layers given by

$$
R_{i j}=\frac{\rho_{j} k_{z i}-\rho_{i} k_{z j}}{\rho_{j} k_{z i}+\rho_{i} k_{z j}} .
$$

From (2.15) it is clear that $R_{B}$ depends solely on the sediment properties of the bottom. Consequently, measurements of the bottom reflection coefficient are often used as a basis for the geoacoustic inverse problem such as in the trace methods and 
the method of Gelfand-Levitan previously discussed. In the stratified shallow-water case, the relationship between $R_{b}\left(k_{r}\right)$ and $g\left(k_{r}\right)$ given by (2.14) shows the explicit dependence of the spectrum on the bottom properties. This relationship is the basis for geoacoustic inverse problems using point source acoustic field data in shallowwater. Of particular interest for the inverse problem is the discrete portion of the wavenumber spectrum $g$ given by (2.14) when the denominator goes to zero. Making the substitution $R_{T}=-1$,

$$
1+R_{B} e^{2 i k_{z} z_{b}}=0
$$

It will be shown that this equation takes the exact form of the characteristic equation for the inhomogeneous depth-dependent wave equation for a waveguide with pressurerelease top and horizontally stratified bottom [28]. Solutions to (2.17) can be used as input data to the perturbative inverse methods.

\subsubsection{Cylindrical coordinates: The Hankel Transform}

A more natural representation of (2.7) for a point source in a stratified waveguide is obtained by transforming the problem to cylindrical coordinates. For an axisymmetric problem, transformation to cylindrical coordinates has the effect of reducing the twodimensional conjugate Fourier transform pair relationship of (2.7) and (2.8) to the one-dimensional Hankel transform pair relationship with conjugate variables $k_{r}$ and $r$. The Cartesian/cylindrical transform relations in the spatial and wavenumber domains are given by the following identities.

$$
\begin{array}{ll}
k_{x}=k_{r} \cos \alpha, & x=r \cos \beta, \\
k_{y}=k_{r} \sin \alpha, & y=r \sin \beta, \\
k_{r}=\sqrt{k_{x}^{2}+k_{y}^{2},} & r=\sqrt{x^{2}+y^{2}},
\end{array}
$$

where the angles $\alpha$ and $\beta$ are as shown in figure (2-2). Using these transforms, 


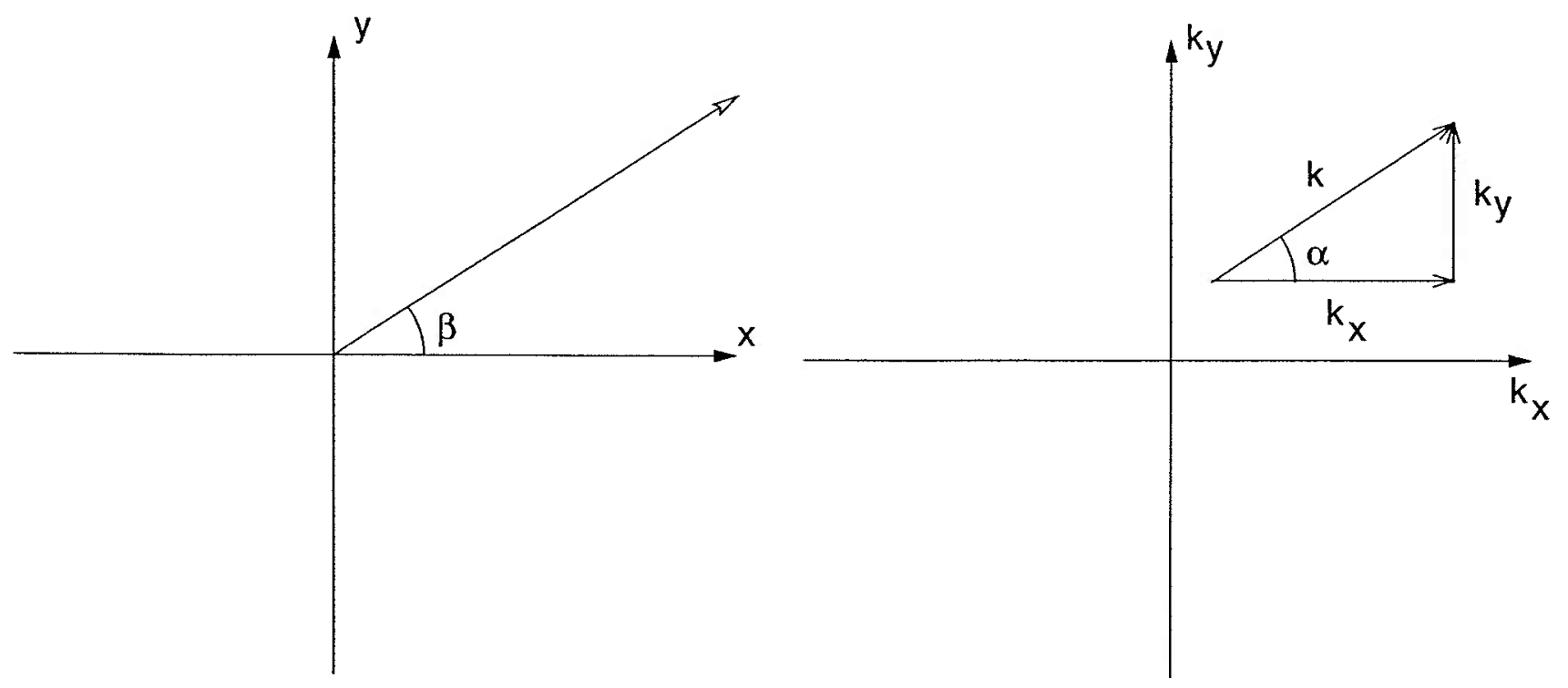

Figure 2-2: Angle definitions for coordinate transforms in Cartesian and wavenumber space

equation (2.7) becomes

$$
p\left(r ; z, z_{o}\right)=\frac{1}{2 \pi} \int_{0}^{\infty} \int_{0}^{2 \pi} g\left(k_{r} ; z, z_{o}\right) e^{i k_{r} r(\cos \alpha-\beta)} k_{r} d k_{r} d \alpha
$$

Using the integral representation for the zero-order Bessel function $J_{o}$,

$$
J_{o}(z)=\frac{1}{2 \pi} \int_{0}^{2 \pi} e^{i z(\cos \alpha-\beta)} d \alpha
$$

equation (2.19) becomes a zero-order Hankel transform which makes up one half of the conjugate transform pair given by,

$$
\begin{gathered}
p\left(r ; z, z_{o}\right)=\int_{0}^{\infty} g\left(k_{r} ; z, z_{o}\right) J_{o}\left(k_{r} r\right) k_{r} d k_{r} \\
g\left(k_{r} ; z, z_{o}\right)=\int_{0}^{\infty} p\left(r ; z, z_{o}\right) J_{o}\left(k_{r} r\right) r d r
\end{gathered}
$$

Using the following identity relating the Bessel function to the Hankel function,

$$
J_{o}\left(k r_{r}\right)=\frac{1}{2}\left[H_{o}^{(1)}\left(k_{r} r\right)+H_{o}^{(2)}\left(k_{r} r\right)\right]
$$


the one-sided integral of (2.21) can be written as the two-sided integral [28]

$$
p\left(r ; z, z_{o}\right)=\frac{1}{2} \int_{-\infty}^{\infty} g\left(k_{r} ; z, z_{o}\right) H_{o}^{(1)}\left(k_{r} r\right) k_{r} d k_{r}
$$

This form is particularly convenient when approximating the Hankel function by its asymptotic form for $k_{r} r \gg 1$ for which the above integral and its inverse take the form of Fourier transforms. Using

$$
H_{o}^{(1)}\left(k_{r} r\right) \sim \sqrt{\frac{2}{\pi k_{r} r}} e^{i\left(k_{r} r-\pi / 4\right)} \quad k_{r} r \gg 1
$$

in (2.23) gives,

$$
p\left(r ; z, z_{o}\right) \sim \frac{e^{-i \pi / 4}}{\sqrt{2 \pi r}} \int_{-\infty}^{\infty} g\left(k_{r} ; z, z_{o}\right) \sqrt{k_{r}} e^{i k_{r} r} d k_{r}
$$

with the inverse,

$$
g\left(k_{r} ; z, z_{o}\right) \sim \frac{e^{i \pi / 4}}{\sqrt{2 \pi k_{r}}} \int_{-\infty}^{\infty} p\left(r ; z, z_{o}\right) \sqrt{r} e^{-i k_{r} r} d r
$$

These expressions are easily incorporated for use on measured acoustic field data where the wavenumber spectrum can be extracted using methods based on the Fast Fourier Transform (FFT) or other computational techniques commonly used in signal processing.

Equations (2.24) and (2.25) are often used as the basis for waveguide characterization and geoacoustic inversion techniques based on spatial measurements of point source acoustic wave fields. 


\subsubsection{Normal Mode Representation}

An alternative solution to the standard wave equation given by (2.4), and written in cylindrical coordinates as [28]

$$
\left[\nabla^{2}+k^{2}(\mathbf{r})\right] p(\mathbf{r})=-4 \pi \frac{\delta(r)}{r} \delta(\theta) \delta\left(z-z_{o}\right)
$$

is obtained through the assumption of a separable solution of the form,

$$
p(r, z)=\sum_{n} a_{n}\left(z_{o}\right) \Psi_{n}(z) R_{n}(r)
$$

where $\mathbf{r}=(r, \theta, z), R_{n}(r)$ and $\Psi_{n}(z)$ are radial and depth eigenfunctions and $a_{n}\left(z_{o}\right)$ is amplitude. The important result here is that for constant-density layers, the $\Psi_{n}$ satisfies the eigenvalue equation

$$
\frac{d^{2} \Psi_{n}(z)}{d z^{2}}+k_{z n}^{2} \Psi_{n}(z)=0 ; \quad k_{z n}^{2}=k^{2}(z)-k_{n}^{2}
$$

along with its boundary conditions, and $k_{n}$ is the eigenvalue for the mode $n$. For a homogeneous fluid layer bounded from above and from below by horizontally stratified media with plane-wave reflection coefficients $R_{T}$ and $R_{B}$, respectively, the homogeneous solution to the depth equation can be written in terms of up-going and down-going plane waves.

$$
\Psi(z)=\Psi_{d}(z)+\Psi_{u}(z)=A e^{i k_{z} z}+B e^{-i k_{z} z}
$$

where $A$ and $B$ are arbitrary constants determined by the boundary conditions. Using this expression, the reflection coefficients at the top and bottom surfaces can be written as

$$
R_{T}=\left.\frac{\Psi_{d}(z)}{\Psi_{u}(z)}\right|_{z=0}=\frac{A}{B}
$$




$$
R_{B}=\left.\frac{\Psi_{u}(z)}{\Psi_{d}(z)}\right|_{z=z_{b}}=\frac{B}{A} e^{-2 i k_{z} z_{b}}
$$

These two equations can then be combined to yield the characteristic equation for the depth dependent equation given by

$$
1-R_{T} R_{B} e^{2 i k_{z} z_{b}}=0 .
$$

Recalling equation (2.14), this is exactly the form of the denominator for the shallowwater depth-dependent Green's function given in equation (2.17) for $R_{T}=-1$. Poles of the Green's function thus correspond exactly to eigenvalues for the depth-dependent eigenvalue equation.

Following the treatment of Frisk [28], the radial component of the solution (2.27) can be shown to be a solution to Bessel's equation with a line source driving term and has the form,

$$
R_{n}(r)=i \pi H_{o}^{(1)}\left(k_{n} r\right)
$$

The modal representation of the acoustic pressure field can then be written,

$$
p(r, z)=i \pi \sum_{n=1}^{\infty} a_{n}\left(z_{o}\right) \Psi_{n}(z) H_{o}^{(1)}\left(k_{n} r\right) .
$$

Finally, solving for $a_{n}(z)$ for $z=z_{o}$ using the closure relation for eigenfunctions of a proper Sturm-Liouville system as,

$$
a_{n}\left(z_{o}\right)=\Psi_{n}^{*}\left(z_{o}\right) / \rho\left(z_{o}\right)
$$

and the modal representation becomes,

$$
p(r, z)=\frac{i \pi}{\rho\left(z_{o}\right)} \sum_{n=1}^{\infty} \Psi_{n}^{*}\left(z_{o}\right) \Psi_{n}(z) H_{o}^{(1)}\left(k_{n} r\right) .
$$


In the far field, the asymptotic form of the Hankel function can be used to obtain.

$$
p(r, z) \sim \frac{\sqrt{2 \pi} e^{i \pi / 4}}{\rho\left(z_{o}\right)} \sum_{n=1}^{\infty} \Psi_{n}^{*}\left(z_{o}\right) \Psi_{n}(z) \frac{e^{i k_{n} r}}{\sqrt{k_{n} r}} .
$$

\subsubsection{Range-Dependent Modal Representation}

In the previous development of the modal solution to the depth-dependent problem, a range-independent medium was assumed. Pierce [57] first introduced the rangedependent formulation for normal modes where he assumed small variations in the propagation medium with range and that individual modes do not transfer energy between one another. Thus, by representing the solution at any given range as a sum of local modes, where the modes satisfy the local boundary conditions, the adiabatic mode formula can be derived and is given by,

$$
p(r, z) \simeq \frac{\sqrt{2 \pi}}{\rho\left(z_{o}\right)} e^{i \pi / 4} \sum_{n=1}^{\infty} \Psi_{n}\left(z_{o}\right) \Psi_{n}(r, z) \frac{e^{i \int_{0}^{r} k_{n}\left(r^{\prime}\right) d r^{\prime}}}{\sqrt{\int_{0}^{r} k_{n}\left(r^{\prime}\right) d r^{\prime}}}
$$

where the integral over $d r$ in the denominator of the mode sum has been included to ensure reciprocity between source and receiver. Through this formulation and application of the transform relations between the depth-dependent Green's function and the complex-pressure field, range-dependent spectra can be extracted by performing the wavenumber integral over local apertures of finite length.

It should be noted that although adiabatic mode theory is a convenient way to represent the range-varying nature of the local modes for a spatially varying waveguide, it is not a necessary condition for the extraction of range-dependent spectra. It will be demonstrated that effective wavenumber estimates can be obtained for regions of locally range-independent environments bounded by regions of the environment where adiabatic mode theory does not apply and mode coupling occurs. Similarly, for the case of weak internal waves in a range-independent waveguide, effective estimates of the propagating modes can be made where mode coupling occurs. In this case 
it will be shown that the higher order modes are relatively stable indicating rangeindependent bottom properties and that energy transfered amongst the lower-order modes is due to range-dependence in the water column.

\subsubsection{Effects Due to Source/Receiver Motion}

In the MOMAX experiments to be described, the source/receiver geometry was dependent on the drift tracks of the individual buoys and/or whether the source was moored or being towed. As such, any effect these configurations might have on the measured wavenumber spectra should be addressed. In the typical range separation experiment, it has been recognized that towed source motion imparts a shift in the measured wavenumber spectrum. Rajan et. al. [67] corrected for this effect in measurements made in the Hudson Canyon area of the Atlantic Ocean, where a source was towed along a radial at $1.5 \mathrm{~m} / \mathrm{s}$. Hawker [38] and Schmidt and Kuperman [73] give detailed analyses of the moving source problem and derive independent expressions for the expected Doppler shifts. Each of their methods will be reviewed here and applied to the MOMAX measurements in a following chapter.

Hawker treats the case for a source moving along a constant trajectory with constant speed past a stationary receiving array. For the geometry in Figure 2-3 he derives a modal solution to the wave equation that includes the geometry and source motion. Through his analysis, he arrives at a modal solution which depends on source velocity and angle to receiver along with modal group velocity.

$$
p(r, z) \sim \frac{\sqrt{2 \pi} e^{i \pi / 4}}{\rho\left(z_{o}\right)} \sum_{n=1}^{\infty} \frac{\Psi_{n}^{*}\left(z_{o}\right) \Psi_{n}(z)}{\sqrt{k_{n}^{o} R}} \exp \left[i k_{n}^{o} R\left(1-\frac{v}{v_{n}^{g}} \sin \theta\right)\right],
$$

where $R$ and $\theta$ are the slant range and angle between source and receiver, $k_{n}^{o}$ is the modal eigenvalue for no source motion, and $v_{n}^{g}$ is the group velocity of mode $n$. Comparing the result of Hawker given by (2.39), to the mode sum for a stationary source given by (2.37), the horizontal wavenumbers in the moving source problem are 


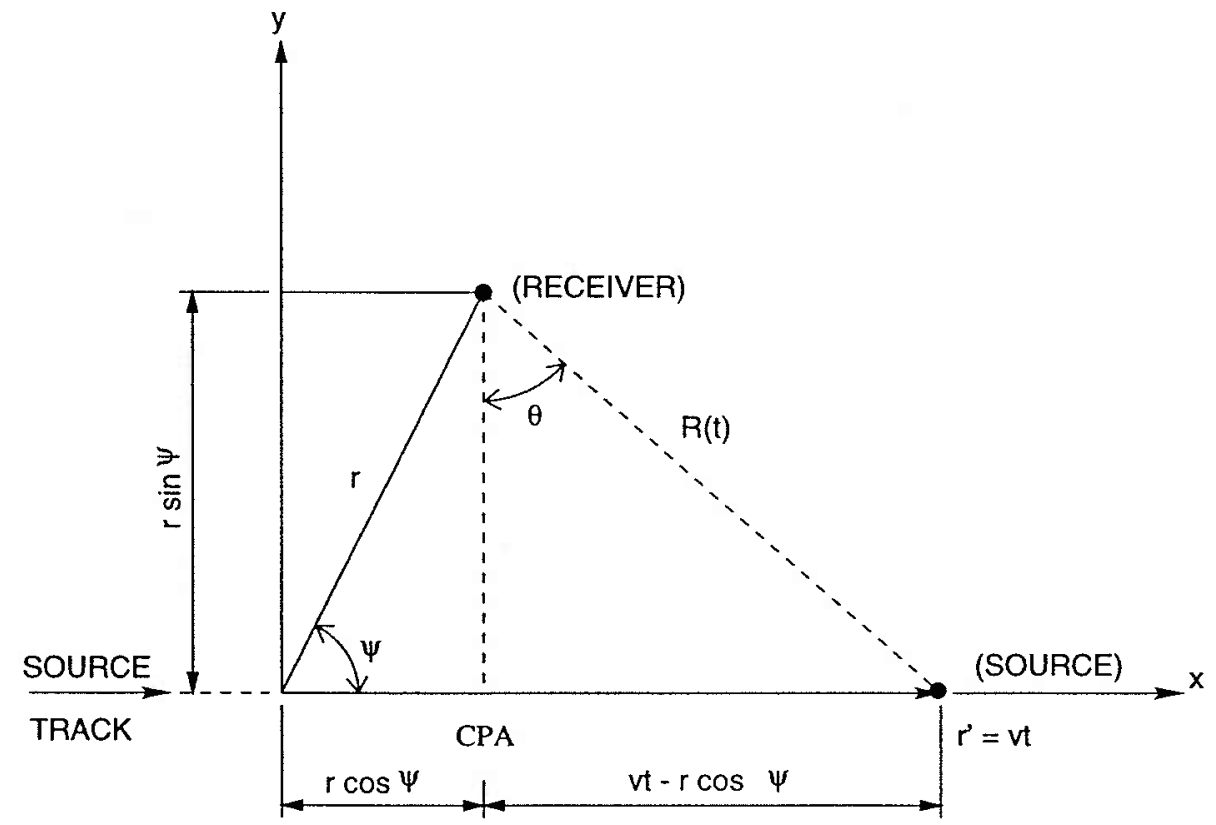

Figure 2-3: Source/Receiver geometry considered by Hawker for moving source problem [38].

shifted by an amount equal to the ratio of the source speed to modal group velocity. For the geometry in figure 2-3, the shifted wavenumbers are given by,

$$
k_{n}^{*} \simeq k_{n}\left(1-\frac{v_{s}}{v_{n}^{g}} \sin \theta\right) .
$$

A later paper by Schmidt and Kuperman [73] treats a similar problem, but allows for more general source/receiver geometry. In their analysis, the source and receiver are constrained to the same horizontal plane, but can move with constant velocity at arbitrary angles with respect to one another as illustrated in figure 2-4. Under these conditions, and assuming a spatially independent horizontally stratified medium, the spectral and modal representations of the pressure field are derived. The Green's function is shown to be a solution to a depth-dependent wave equation as in the stationary case of (2.6) but evaluated at a Doppler shifted frequency given by

$$
\omega^{*}=\omega+\mathbf{k}_{\mathrm{r}} \cdot \mathbf{v}_{\mathrm{s}},
$$




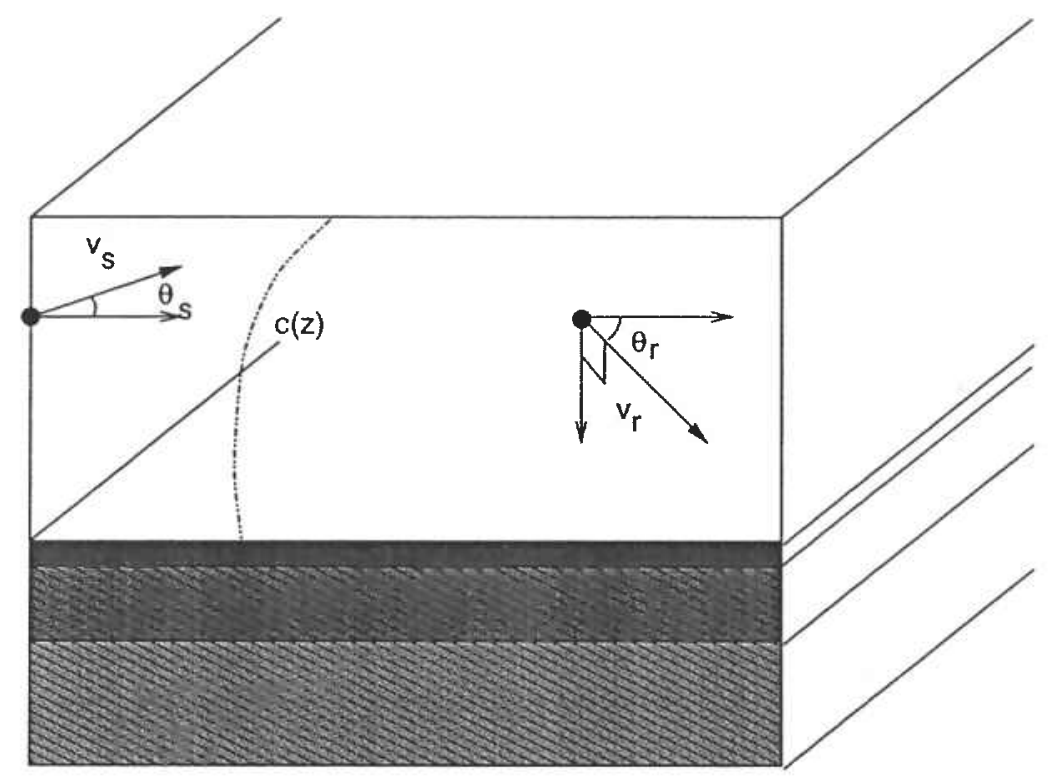

Figure 2-4: Geometry used by Schmidt and Kuperman for analysis of Doppler shifts due to source/receiver motions [73].

where $v_{s}$ is the source velocity. The time-domain solution to the wave equation for a moving source is then shown to be given in terms of a depth-dependent Green's function that depends on the source motion. The receiver motion is introduced independently and is shown to introduce a frequency shift in the exponential term of the time-domain solution, but not in the integration kernel. For both a moving source and moving receiver, the time-domain solution to the wave equation can be written as $[73]$

$$
p\left(\mathbf{r}_{\mathbf{o}}+\mathbf{v}_{\mathbf{r}} t, z, t\right) \sim \frac{1}{\rho \sqrt{2 \pi}} \int g\left(k_{r}, z ; \omega_{o}+\mathbf{k}_{\mathbf{r}} \cdot \mathbf{v}_{\mathbf{s}}\right) e^{-i\left[\left[\omega_{o}+k_{r} \cdot\left(\mathbf{v}_{\mathbf{s}}-\mathbf{v}_{\mathbf{r}}\right)\right] t-\mathbf{k}_{\mathbf{r}} \cdot \mathbf{r}_{\mathbf{o}}\right]} d^{2} \mathbf{k}_{\mathbf{r}}
$$

where the receiver is moving with a velocity vector $\mathbf{v}_{\mathbf{r}}$ given by $\mathbf{r}=\mathbf{r}_{\mathbf{o}}+\mathbf{v}_{\mathbf{r}} t$. This result of Schmidt and Kuperman shows that the effects of moving sources and receivers is not reciprocal. Only source motion causes a Doppler frequency shift in the propagating modes of the field for a harmonic source, while both source and receiver motion contribute to the Doppler wavenumber shift in the phase term. In analysis of the experimental data for this work, observations of the modal shift predicted by these models will be explored and a method for extracting the group velocity directly 
from measurements considered.

\subsection{Inverse Problem}

The preceding sections describe the forward problem for predicting acoustic propagation as a solution to the depth-dependent wave equation given the boundary conditions. The inverse problem of interest is to determine the properties of the bounding media through measurements of the propagating acoustic field. The data used in the inverse methods to be described is the spectral representation of the propagating acoustic field. It was shown in equation (2.14) how this representation contains information about the bottom properties of interest.

\subsubsection{Perturbative Inversion}

The perturbative inverse method is an iterative method that is based on making small perturbations to a starting background model. Here, the formalism of Rajan et al. [62] is followed for formulating the problem as a Fredholm integral equation that can be solved using linear inverse theory.

The starting point is the solution to the depth-dependent wave equation given a predetermined environmental model. The normal mode solution for the initial problem, assuming a piecewise constant density profile is written,

$$
\left(\frac{d^{2}}{d z^{2}}+k^{(0) 2}(z)\right) \Psi_{m}^{(0)}(z)=k_{m}^{(0) 2} \Psi_{m}^{(0)}(z)
$$

where,

$$
k^{(0)}(z)=\omega / c_{o}(z)
$$

is the wavenumber for the background sound speed profile $c_{o}(z), \Psi_{m}^{(0)}(z)$ is the $m$ th normal mode function, and $k_{m}^{(0)}$ is the $m$ th mode eigenvalue for the background problem. Given this model, a perturbation in the background sound speed model by $\Delta c(z)$ 
gives the perturbed wavenumber,

$$
k(z)=\omega /\left[c_{o}(z)+\Delta c(z)\right]
$$

This perturbation in wavenumber yields a corresponding perturbation to the eigenvalues that when linearized can be expressed by,

$$
\Delta k_{m}=\left(2 k_{m}^{(0)}\right)^{-1}\left\langle\Psi_{m}^{(0)}|H| \Psi_{m}^{(0)}\right\rangle
$$

where brackets denote integration over depth, and $H$ is the wavenumber perturbation squared and linearized.

$$
\begin{aligned}
H & \equiv \Delta k^{2}(z)=k^{2}(z)-k^{(0) 2}(z) \\
& =\frac{\omega^{2}}{c_{o}^{2}(z)}\left[\left(1+\frac{\Delta c(z)}{c_{o}(z)}\right)^{-2}-1\right] \\
& \cong-k^{(0) 2}\left(2 \Delta c / c_{o}\right) \text { for } \Delta c / c_{o}<<1
\end{aligned}
$$

Substituting equation (2.47) into (2.46) and expressing the depth integral indicated by the brackets explicitly, the mode number perturbations can be written as the integral equation,

$$
\Delta k_{m}=\frac{1}{k_{m}^{(0)}} \int_{0}^{\infty} \rho_{o}^{-1}(z)\left|\Psi_{m}^{(0)}(z)\right|^{2} k^{(0) 2}(z) \frac{\Delta c(z)}{c_{o}(z)} d z
$$

In this form, a given small perturbation in the background sound speed profile results in a small change in the modal eigenvalues. The iterative perturbative inversion method thus makes small perturbations to the background sound speed profile and compares the resulting eigenvalues to the measured input values. The background profile is updated iteratively until the eigenvalues from the updated profile agree with the measured input data.

Rajan et. al. [62] details three methods for solution of the integral equation given by (2.48) from which the regularization method is shown here. This method applies a 
smoothness constraint on the solution of the integral equation by requiring the second derivative of the sound speed to be continuous across layer interfaces in the sediment. The smoothness measure thus stated is defined as,

$$
S(\Delta c(z))=\int_{0}^{a}\left(\frac{d^{2} \Delta c(z)}{d z^{2}}\right)^{2} d z
$$

Using the above constraint, the integral equation (2.48) is discretized and recast as an augmented least-square problem with the objective of minimizing a cost function given by the squared difference of $\Delta k_{m}$ as given below. Input data for the problem is the difference between measured eigenvalues as determined by taking the Hankel transform of complex pressure and the eigenvalues calculated for the background model. The data is a vector, $\mathbf{d}$, whose length is determined by the number of modes with elements given by

$$
d_{i}=\Delta k_{m}=k_{m}-k_{i}
$$

where $k_{m}$ are the measured eigenvalues and $k_{i}$ are the mode eigenvalues for the background problem. The unperturbed values in the integral equation (2.48) can be expressed as a matrix, $\mathbf{G}$, with elements,

$$
G_{i j}=\frac{\omega^{2}}{\rho_{o} c_{o}^{3}}\left|\Psi_{m}^{(0)}\right|^{2}
$$

where $i$ and $j$ refer to mode number and layer depth indices, respectively. The smoothness constraint is then expressed by the double difference equation given by,

$$
S(\Delta \mathbf{c})=\sum_{j}\left(\Delta c_{j+1}-2 \Delta c_{j}+\Delta c_{j-1}\right)^{2}=\Delta \mathbf{c}^{\mathbf{T}} \mathbf{H} \Delta \mathbf{c}
$$


with

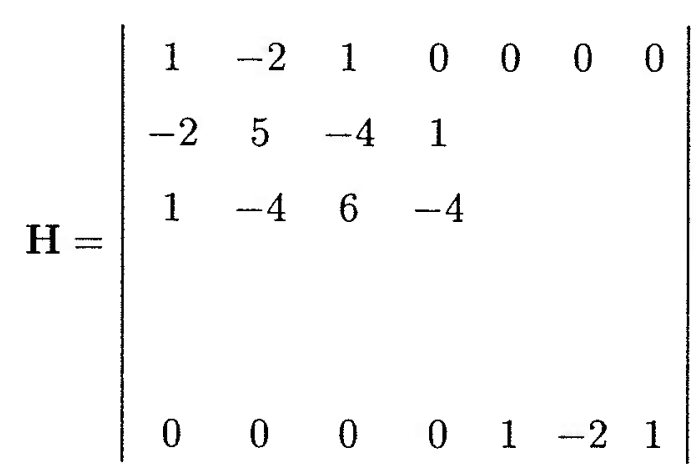

In the above, $S$ is a vector of length depending on the number of depth layers the problem has been discretized with, $G$ is an $n \times m$ matrix corresponding to the number of modes and number of layers. The solution is then found by determining the $\Delta c$ that minimizes the following equation for $l$ given by

$$
l=\lambda \Delta \mathbf{c}^{T} \mathbf{H} \Delta \mathbf{c}+(\mathbf{d}-\mathbf{G} \Delta \mathbf{c})^{T}(\mathbf{d}-\mathbf{G} \Delta \mathbf{c})
$$

where $\lambda$ is a Lagrange multiplier representing the amount of smoothness applied. Differentiating (2.54) with respect to each element of $\Delta \mathbf{c}$, the problem takes the form

$$
\Delta \mathrm{c}=\left(\mathrm{G}^{\mathrm{T}} \mathrm{G}+\lambda \mathbf{H}\right)^{-1} \mathrm{G}^{\mathrm{T}} \mathbf{d}
$$

In the above, when $\lambda=0$, no smoothness constraints are applied and the equation reduces to the standard least square solution for $\Delta \mathbf{c}$ given by

$$
\Delta \mathbf{c}=\mathrm{G}^{-1} \mathbf{d}
$$

Thus stated, for a given $\Delta k_{m}$, the minimization problem is solved to determine a perturbation to the background model by $\Delta c$. The cost function is evaluated for the new background model and iteration proceeds until agreement is obtained between the updated model and the input data. 


\subsubsection{Gelfand-Levitan Method}

The Gelfand-Levitan method is an exact method for geoacoustic inversion that is based on the analogous representation of the depth-dependent acoustic equation with the time-independent Schrödinger equation of quantum scattering theory. The timeindependent inhomogeneous wave equation for the depth-dependent variable $u\left(k_{r}, z\right)$ can be written as,

$$
\left(\frac{d^{2}}{d z^{2}}+k^{2}-k_{r}^{2}\right) u\left(k_{r}, z\right)=0
$$

By making a simple wavenumber translation given by $\mu^{2}=k_{o}^{2}-k_{r}^{2}$, and $q(z)=$ $k_{o}^{2}-k^{2}(z)$, the equation (2.57) can be written,

$$
\left(\frac{d^{2}}{d z^{2}}+\mu^{2}-q(z)\right) u(\mu, z)=0
$$

with boundary condition $u(\mu, 0)=0$. This is easily seen to take the form,

$$
\left\{\frac{d^{2}}{d z^{2}}+[E-V(z)]\right\} u(E, z)=0
$$

which is the time-independent Schrödinger equation where $E=\mu^{2}$ is the "energy" and $V(z)=q(z)$ is the "potential". In the above and for what follows, it has been assumed that the density is held constant over all depths. This requirement will be relaxed in a later section.

Considering the model shown in figure 2-5, and following closely the development by Chadan and Sabatier [12], the scattering solution to this full-line equation is governed by the asymptotic boundary conditions for $z \rightarrow \pm \infty$ :

$$
\begin{gathered}
u_{ \pm}(z) \sim \frac{e^{ \pm i \mu z}}{\sqrt{2 \pi}}+R_{\mp}(\mu) \frac{e^{\mp i \mu z}}{\sqrt{2 \pi}}, \quad z \rightarrow \mp \infty \\
u_{\mp}(z) \sim T_{ \pm}(\mu) \frac{e^{ \pm i \mu z}}{\sqrt{2 \pi}}, \quad z \rightarrow \pm \infty .
\end{gathered}
$$

The two independent solutions $u_{ \pm}(z)$ correspond to plane waves emanating from 


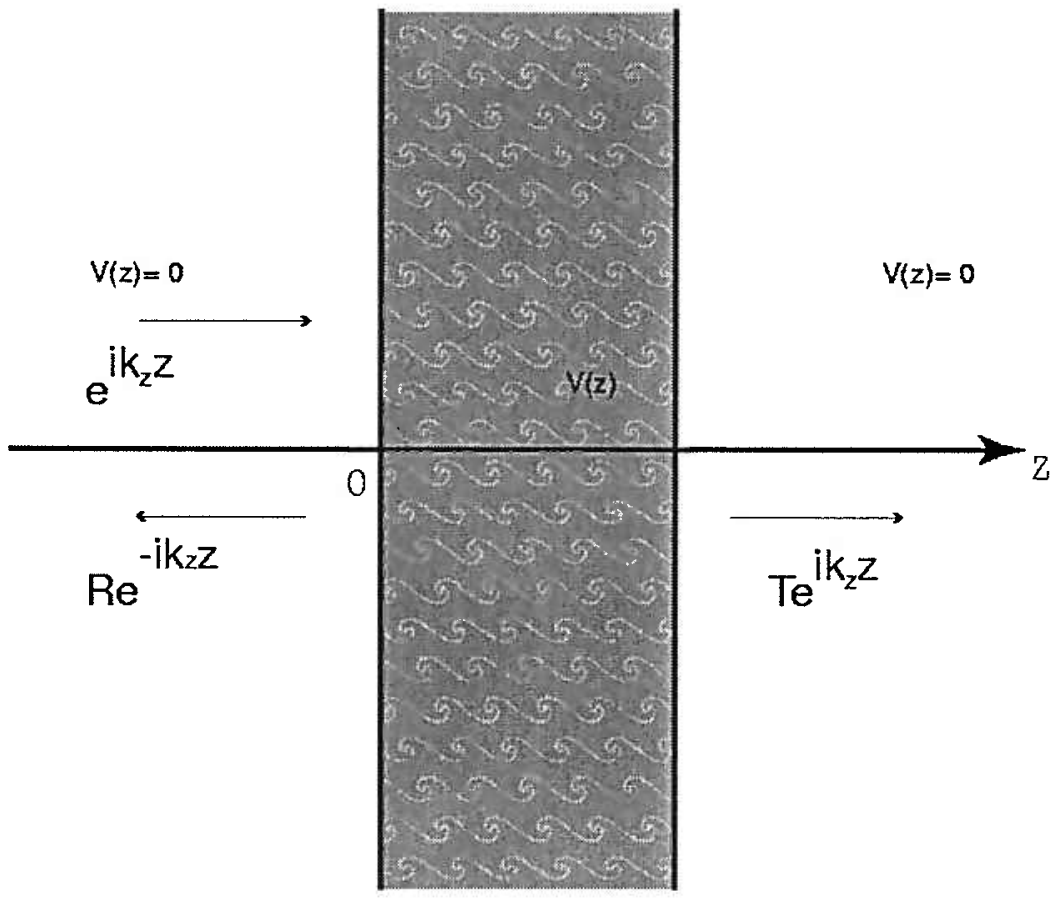

Figure 2-5: 1-Dimensional inverse scattering problem model. Inverse problem is to determine the potential $\mathrm{V}(\mathrm{z})$ of the scattering region.

$z=\mp \infty$ where $R_{\mp}$ are the reflection coefficients in the $\mp z$ directions and $T_{ \pm}$are the transmission coefficients in the $\pm z$ directions. The reflection and transmission coefficients make up the full scattering matrix of the Schrödinger equation

$$
S(\mu)=\left(\begin{array}{cc}
T_{+}(\mu) & R_{-}(\mu) \\
R_{+}(\mu) & T_{-}(\mu)
\end{array}\right) .
$$

Assuming the end potentials as $z \rightarrow \pm \infty$ are both equal to zero, the full scattering matrix can be determined from $R_{-}(\mu)$.

Thus, the geoacoustic inverse problem becomes one of determining the potential $q(z)$ from the reflection coefficient $R_{-}(\mu)$ including information from both the discrete and continuous spectrum. Note that for $k_{r}>k_{\infty}, \mu$ becomes imaginary. The energy $\mu^{2}$ is always real and is positive for $\mu$ real. The potential $q(z)$ is in general positive.

Chadan and Sabatier[12] continue with the solution of the one-dimensional inverse 
scattering problem making the following assumption about the potential:

$$
\int_{-\infty}^{\infty}(1+|z|) V(z) d z<\infty
$$

A solution to the inverse problem can be found using the Fourier transform of the reflection coefficient,

$$
\hat{R}(z)=\frac{1}{2 \pi} \int_{-\infty}^{\infty} R(\mu) e^{-i k_{z} z} d k_{z},
$$

Where $R(\mu)=R_{-}(\mu)$. This expression is inserted into the Gelfand-Levitan type equation,

$$
K(z, y)+\hat{R}(z+y)+\int_{-\infty}^{z} \hat{R}\left(z^{\prime}+y\right) K\left(z, z^{\prime}\right) d z^{\prime}=0, \quad y<z
$$

which can be solved by relatively straightforward numerical methods. It should be noted that Deift and Trubowitz make the same assumptions about the potential to solve an identical inverse problem in terms of their trace formula. Merab [50] derives the same equation for unequal end potentials at $z= \pm \infty$.

The theory, as stated, is quite straightforward and requires only the plane-wave reflection coefficient as input data to the inversion. However, there are several caveats that must be addressed before application of the Gelfand-Levitan method can be applied in practice. It was already mentioned that the development assumed the density to be constant in the problem. This assumption allows the plane-wave reflection coefficient to be expressed solely as a function of sound speed ratios at the layer interfaces. However, it was shown in equation (2.16) that for the acoustic problem, the sound speed and density are coupled for determining the reflection coefficient. Merab [50] gives a derivation for the case of continuous variation of density in the sediment, but does not address the jump discontinuity at the water-bottom interface. This and other practical issues associated with solving the G-L integral equation will be discussed along with some numerical examples in chapter 5 of the thesis. A goal is 
to address issues relating to the application of the method to acoustic pressure data measured at sea. 


\section{Chapter 3}

\section{Wavenumber Estimation}

\section{Techniques}

\subsection{Introduction}

In this chapter practical aspects of extracting the discrete horizontal wavenumber spectrum from range-dependent acoustic data are considered. Of particular importance are problems where the expected local eigenvalues are closely spaced in the wavenumber domain. In these cases, classical spectral analysis methods show the limit of resolution for detection of two closely spaced wavenumber values to be inversely proportional to the data length. In cases where data are limited to a given aperture, or the environment changes rapidly, it may be impossible to resolve individual modes. This is demonstrated in figures $3-1-3-3$ for a $50 \mathrm{~Hz}$ complex pressure signal measured on a 5 meter grid where spectra are determined for apertures of 1000 $\mathrm{m}$ and $2000 \mathrm{~m}$ using both classical and high-resolution methods. More details about the field and the resulting wavenumber estimates are discussed later in this chapter. 


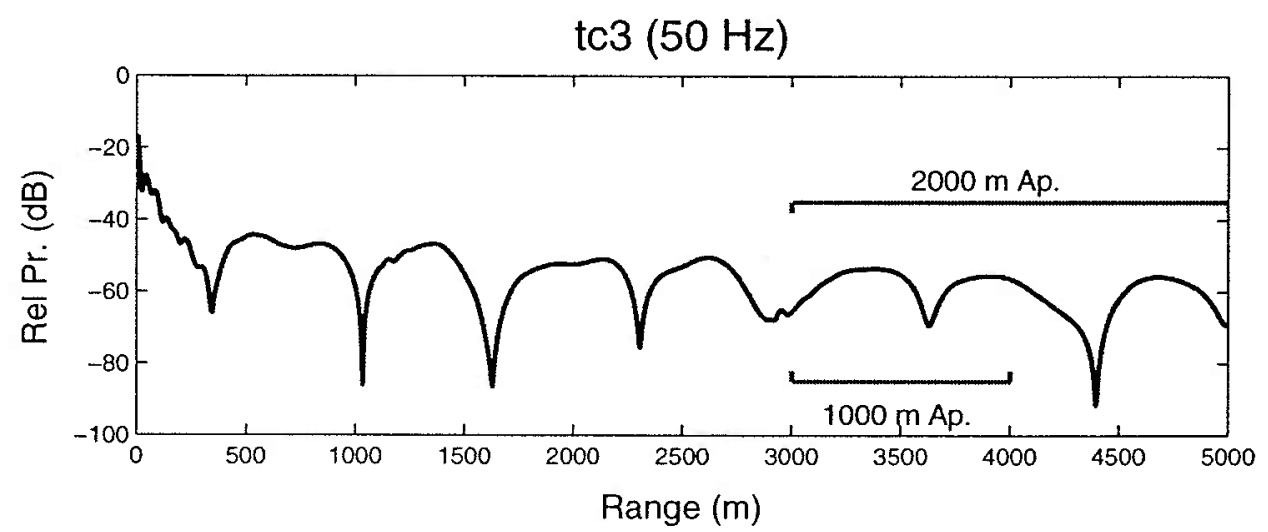

Figure 3-1: $50 \mathrm{~Hz}$ synthetic pressure field data for waveguide with unknown rangedependent sediment properties. $1000 \mathrm{~m}$ and $2000 \mathrm{~m}$ data segments are shown as used for making wavenumber estimates. 


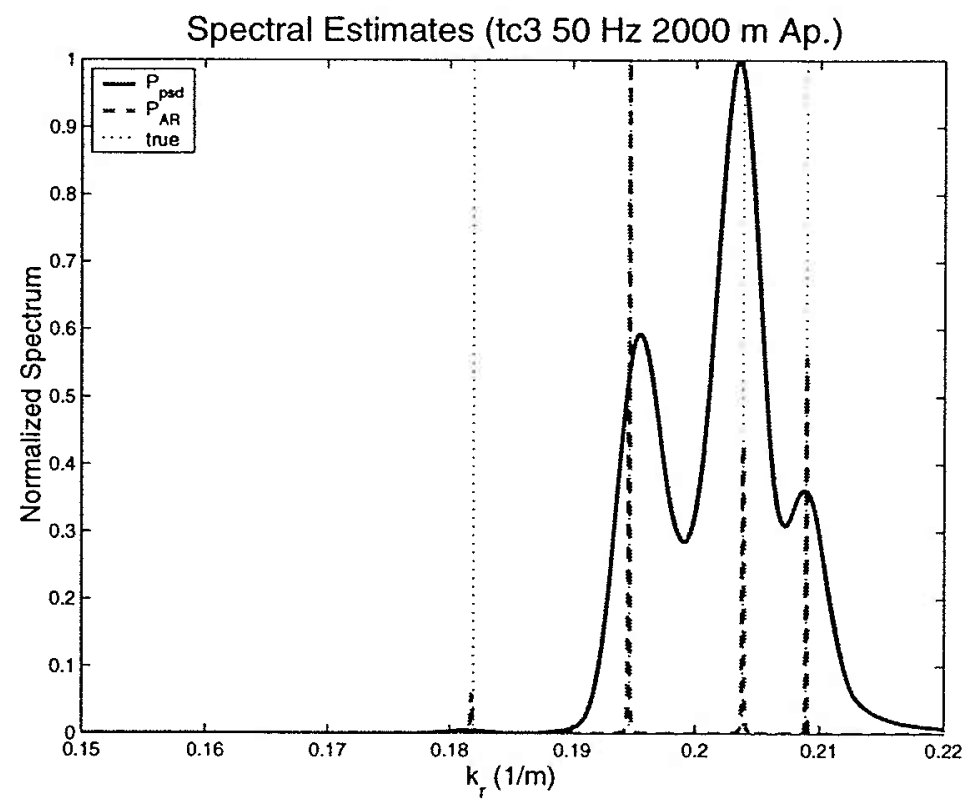

Figure 3-2: Comparison of spectral estimates for $2000 \mathrm{~m}$ data aperture. Classical PSD result is solid, high-resolution result is dashed, and true wavenumber values are dotted.

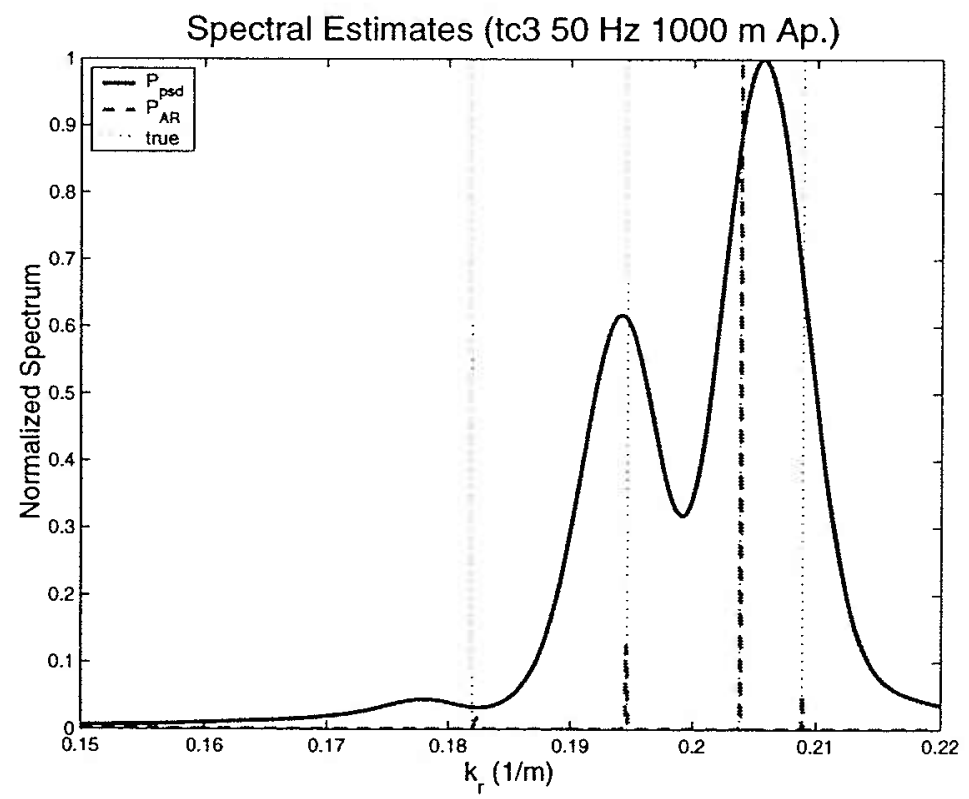

Figure 3-3: Comparison of spectral estimates for $1000 \mathrm{~m}$ data aperture. Classical PSD result is solid, high-resolution result is dashed, and true wavenumber values are dotted. 
In making spectral estimates based on classical methods, Matlab's [60] psd function was used with a Hanning window applied to the full data aperture and the number of FFT points specified as 16384. The High-resolution estimates were made using the modified-covariance autoregressive (AR) estimator of Marple [49] with AR model orders of 20 and 30 for the $2000 \mathrm{~m}$ and $1000 \mathrm{~m}$ apertures, respectively. Having estimated the AR model coefficients, the spectra were determined on a 16384 point wavenumber grid. Full details of the AR estimator are discussed in Marple [49] with a summary provided below. Before proceeding, it should be emphasized that for this work the data to be extracted from the wavenumber spectral plots is the location of the wavenumber peaks, not the amplitudes. Recognizing that the variance in peak levels for AR spectral estimates can be large compared to other methods [4], observed discrepancies in spectral amplitudes at the resolved frequencies between the classical and high-resolution methods will largely be ignored. This statement in effect reduces the problem from that of spectral estimation to one of parameter estimation where the parameters being sought are horizontal wavenumbers.

Using the above statements as a guide, wavenumber estimates can be made by identifying the peak locations of the spectral estimates for both the $2000 \mathrm{~m}$ and 1000 $\mathrm{m}$ apertures shown in figures 3-2 and 3-3. The location of the true wavenumbers are plotted as the dotted lines in each of the figures. From the plots, it is clear the high-resolution method is capable of determining the four discrete wavenumber components for both the short and long apertures. In contrast, the classical result is not able to resolve the four wavenumber components for the short aperture and shows a bias in the peak locations for the long aperture.

In the next section, the high-resolution method is described and characterized with emphasis on its ability to detect discrete wavenumbers in the presence of noise. The estimator will then be used to estimate the range-dependent wavenumber content of the data shown in figure 3-1. 


\subsection{High-resolution wavenumber estimation}

The problem of wavenumber estimation for point-source acoustic data is intimately related to the problem of frequency estimation from a finite number of noisy discretetime signal measurements. For this work, spectral estimators, typically associated with estimating functions of frequency, are to be used as parameter estimators, where the parameters are horizontal wavenumbers. In this context, an estimator is desired for identifying and localizing peaks in the depth-dependent Green's function that correspond to the discrete horizontal wavenumbers of propagating modes using short data apertures. The signal model is deterministic with added noise and the requirement of short data apertures, suggests the use of modern spectral estimation techniques for extraction of the wavenumber content. In particular, a method based on autoregressive spectral analysis was chosen for its high-resolution frequency estimation property [4] [71] [49]. This method will be investigated numerically by first applying it to time-series data for estimating frequency and then applying it to the wavenumber estimation problem of shallow-water acoustics. Where appropriate, reference will be made to the statistical characteristics of the results based on the data. In particular, the resolution of closely space frequencies or wavenumbers will be investigated and the variance of the estimates will be considered for signals with additive noise. The resolution will be looked at with regard to the theoretical resolution of AR based frequency estimators given by Marple [49],

$$
\delta f=\frac{1.03}{T p\left[S N R_{\text {lin }}(p+1)\right]^{0.31}}
$$

where $\delta f$ is the frequency resolution, $T$ is the sample interval in seconds, $p$ is AR model order, and $S N R_{\text {lin }}$ is the signal-to-noise ratio in linear units. The variance of AR spectral estimators used for frequency estimation was examined by Sakai [71] and found to be proportional to the inverse of the product of data length and the square of SNR, where SNR is again given in linear units. The results of Marple and 
Sakai show that both resolution and variance of AR frequency estimates depend on noise level. Although the dependence on noise level will not be explicitly studied with respect to the classical estimators, it should be clear that as SNR decreases, the advantage of using the AR estimator will decrease. In particular, as SNR decreases, the denominator of (3.1) decreases and the number of AR model parameters has to be increased to achieve the same resolution. Further, for low enough SNR, even for high model orders, the resolution will approach the classical limit based on data record length and the advantages of using an AR based estimator are lost. Therefore, SNR is a prime consideration when applying the AR methods to real data.

This thesis work was partially motivated by the work of Rajan and Bhatta [65] where other high-resolution frequency estimators were studied. This work follows their format beginning with frequency estimation for a signal comprised of a single tone plus noise and continuing on to the acoustic problem of horizontal wavenumber estimation. The single-tone problem was treated by Rife and Boorstyn [69] where they derived the Cramer-Rao (CR) bound for the estimation of frequencies in a noisy signal. The CR bound is the best estimate that can be made for a given parameter using an unbiased estimator on the available data. The signal model for their analysis was of the form,

$$
x(t)=\sum_{i=1}^{k} b_{i} e^{i\left(\omega_{i} t+\theta_{i}\right)}+w(t)
$$

where the sum is over a finite number of frequencies, $\omega$, indexed by $k, b_{i}$ are the amplitudes, $\theta_{i}$ are the phases, $w(t)$ is the observation noise, and $t$ is time. Assuming a constant sampling rate of $F_{s}=1 / T \mathrm{~Hz}$ with the first sample taken at $t_{o}$, the continuous signal model can be written in discrete form using the convention for discrete time given by

$$
t_{n}=t_{o}+n T=\left(n_{o}+n\right) T
$$


A single sample is then given at time $t_{n}$ by

$$
x[n]=x\left(t_{n}\right)=\sum_{i=1}^{k} b_{i} e^{i\left(\omega_{i} t_{n}+\theta_{i}\right)}+w\left(t_{n}\right) .
$$

For their work, and the discussion that follows, the noise is independent Gaussian white noise with zero mean and variance $\sigma^{2}$. When estimating a parameter, the mean-squared error is given by,

$$
m s e\{\hat{\alpha}\}=\mathcal{E}\{|\alpha-\hat{\alpha}|\}^{2}=\operatorname{var}\{\hat{\alpha}\}+|B(\hat{\alpha})|^{2},
$$

where $\alpha$ is the true parameter value, $\hat{\alpha}$ is the estimate, $\mathcal{E}$ is the expected value operator, and $B$ is the bias. The CR bound for an unbiased estimator, defined as the mse with $B(\hat{\alpha})=0$, gives a minimum value for the variance that can be obtained for parameter estimates using the given data. For a single-tone, $k=1$, and known initial phase, $\theta$, the CR bound is given by [69],

$$
m s e\{\hat{\omega}\} \geq \operatorname{var}\{\hat{\omega}\}=\frac{\sigma^{2}}{b^{2} T^{2}\left(n_{o}^{2} N+2 n_{o} P+Q\right)},
$$

where, $b$ is the amplitude, $n_{o}$ is the number of the first sample, $N$ is the number of samples, and $P$ and $Q$ are defined as,

$$
\begin{gathered}
P=\sum_{n=0}^{N-1} n=\frac{N(N-1)}{2} \\
Q=\sum_{n=0}^{N-1} n^{2}=\frac{N(N-1)(2 N-1)}{6} .
\end{gathered}
$$

This expression was used as a measure of comparison by Rajan and Bhatta [65] for determining the frequency content of noisy signals using eigenanalysis-based frequency estimators. In the next section, a parametric model based AR spectral estimator will be described. Results for estimating a single frequency using the AR estimator will 
be compared to the CR bound above with $n_{o}=0$ and with the results of Rajan and Bhatta. The estimator is also applied to estimate temporal signals with two closely spaced frequency components and the related problem of estimating horizontal wavenumber content for shallow-water acoustic data. The multiple tone results are compared to the theoretical results of Marple and Sakai along with the numerical results of Rajan and Bhatta [65] where the above bound for a single tone defined by Rife and Boorstyn does not apply.

\subsubsection{Parametric Modeling Approach to Spectral Estimation}

Model based approaches to spectral estimation make use of a parametric representation of the data to determine the spectrum. It is an approach based on the assumption that the measured data can be related to the input data through a rational transfer function. The data model is represented by the linear difference equation,

$$
x_{n}=\sum_{l=0}^{q} b_{l} w_{n-l}-\sum_{k=1}^{p} a_{k} x_{n-k}
$$

where $\left\{w_{n}\right\}$ is the input driving sequence with associated parameters $b_{l}$, and $\left\{x_{n}\right\}$ is the output sequence with parameters $a_{k}$ along with their respective $z$-transforms given by,

$$
\begin{aligned}
& A(z)=\sum_{m=0}^{p} a_{m} z^{-m}, \\
& B(z)=\sum_{m=0}^{q} b_{m} z^{-m} .
\end{aligned}
$$

For the given input and output sequences, the transfer function, $H(z)$, is simply,

$$
H(z)=\frac{B(z)}{A(z)}
$$


From these relationships, the power spectrum at the output of a linear filter, $P_{x}(z)$, is related to the input process, $P_{n}(z)$, by

$$
P_{x}(z)=H(z) H^{*}\left(1 / z^{*}\right) P_{n}(z)=\frac{B(z) B^{*}\left(1 / z^{*}\right)}{A(z) A^{*}\left(1 / z^{*}\right)} P_{n}(z)
$$

where ${ }^{*}$ denotes the complex-conjugate. For a white-noise Gaussian process with variance $\sigma^{2}$ evaluated along the unit circle, $z=\exp (i 2 \pi f T)$ for $-F_{s} / 2 \leq f \leq F_{s} / 2$, $P_{x}$ becomes

$$
P_{\text {model }}(f)=P_{x}(f)=\sigma^{2} T|\mathcal{B}(f) / \mathcal{A}(f)|^{2},
$$

where $\mathcal{A}(f)=A(\exp [i 2 \pi f T])$ and $\mathcal{B}(f)=B(\exp [i 2 \pi f T])$.

In the given context, model based spectral estimation requires three steps that include selection of the time-series model, estimation of the model parameters, and calculating the theoretical power spectral density (PSD) from the resulting model. The discussion above and the derivation of model based methods and other modern estimation techniques are reviewed by Kay and Marple [42] with practical applications and computer algorithms presented in Marple [49]. In any of these methods, the goal is increased resolution and fidelity of frequency estimates compared to the classical methods. The success of any given method relies on the ability to fit the assumed model to the data with few parameters. In this work, a model was sought that could determine a reliable estimate of the peak locations corresponding to the discrete wavenumber spectrum expected for the depth-dependent Green's function for shallow water. This requirement is met by a transfer function that can be represented as an all-pole model for the spectrum where peaks occur at the pole locations.

\subsubsection{Autoregressive Spectral Estimation}

The Autoregressive spectral estimator is defined by an all-pole model for the rational transfer function mentioned above. For this case, the first term on the right for the 
linear-predictor model given by (3.9) reduces to $b_{0}=1$ and the data model becomes,

$$
x_{n}=-\sum_{k=1}^{p} a_{k} x_{n-k}+w_{n}
$$

This model is a strictly autoregressive process of order $p$ where the output sequence $x_{n}$ is a linear regression on itself with error terms $w_{n}$. The present value of the process is a weighted sum of past values with a noise term. Using this model, the spectral representation given by (3.14) reduces to,

$$
\mathcal{P}_{A R}(f)=\frac{\sigma^{2} T}{|\mathcal{A}(f)|^{2}}
$$

This equation can be written explicitly in terms of the model parameters $a_{k}$ to show the all-pole nature of the spectral estimate, where peaks occur when the denominator goes to zero.

$$
\mathcal{P}_{A R}=\frac{\sigma^{2} T}{\left|1+\sum_{k=1}^{p} a_{k} \exp (-i 2 \pi f k T)\right|^{2}} .
$$

Given the above relationship, an estimate of the spectrum is obtained by determining the AR parameters, $a_{k}$ and $\sigma^{2}$. This is done formally by relating the AR parameters to the autocorrelation function of the data through the Yule-Walker equations [42].

For the signal model, $x_{n}$, the autocorrelation function, $R_{x x}(k)$ is, $R_{x x}(k)=\mathcal{E}\left[x_{n+k} x_{n}^{*}\right]=\mathcal{E}\left[x_{n}^{*}\left(-\sum_{l=1}^{p} a_{l} x_{n-l+k}+w_{n+k}\right)\right]=-\sum_{l=1}^{p} a_{l} R_{x x}(k-1)+\mathcal{E}\left[w_{n+k} x_{n}^{*}\right]$

The correlation between the signal and noise in the second term of the last expression 
of (3.18) can be found by,

$$
\begin{aligned}
\mathcal{E}\left[w_{n+k} x_{n}^{*}\right] & =\mathcal{E}\left[w_{n+k} \sum_{l=0}^{\infty} h_{l}^{*} w_{n-l}^{*}\right] \\
& =\sum_{l=0}^{\infty} h_{l}^{*} \sigma^{2} \delta_{k+l} \\
& =\sigma^{2} h_{-k}^{*} \\
& = \begin{cases}0, & \text { for } k>0 \\
h_{o}^{*} \sigma^{2}, & \text { for } k=0\end{cases}
\end{aligned}
$$

where it was assumed that $H(z)$ is a causal and stable filter and $\delta_{m}$ is the delta function, with $\delta_{m}=1$ for $m=0$ and 0 otherwise. Imposing the asymptotic limit at $z \rightarrow \infty$ on the transfer function gives, $h_{o}=\lim _{z \rightarrow \infty} H(z)=1$, and yields the Yule-Walker equations given by,

$$
R_{x x}(k)= \begin{cases}-\sum_{l=1}^{p} a_{l} R_{x x}(k-l), & \text { for } k>0 \\ -\sum_{l=1}^{p} a_{l} R_{x x}(-l)+\sigma^{2}, & \text { for } k=0\end{cases}
$$

Given the Yule-Walker equations, the AR parameters can be determined for a given model order $p$ by solving $p$ equations from (3.20) for $k>0$ and then finding $\sigma^{2}$ for $k=0$. In solving the Yule- Walker equations the model order $p$ is a free parameter selected by the user. Criteria for model order selection will be discussed later.

With the model order selected, the Yule-Walker equations can be expressed in matrix form as

$$
\left[\begin{array}{cccc}
R_{x x}(0) & R_{x x}(-1) & \cdots & R_{x x}(-(p-1)) \\
R_{x x}(1) & R_{x x}(0) & \cdots & R_{x x}(-(p-2)) \\
\vdots & \vdots & \ddots & \vdots \\
R_{x x}(p-1) & R_{x x}(p-2) & \cdots & R_{x x}(0)
\end{array}\right]\left[\begin{array}{c}
a_{1} \\
a_{2} \\
\vdots \\
a_{p}
\end{array}\right]=-\left[\begin{array}{c}
R_{x x}(1) \\
R_{x x}(2) \\
\vdots \\
R_{x x}(p)
\end{array}\right]
$$

The autocorrelation matrix above, $\mathbf{R}_{x x}$ is Hermitian $\left(\mathbf{R}_{x x}^{H}=\mathbf{R}_{x x}\right)$, Toeplitz (equal diagonal elements), and is generally positive definite. Further, (3.21) can be augmented 
to include the $k=0$ equation and becomes,

$$
\left[\begin{array}{cccc}
R_{x x}(0) & R_{x x}(-1) & \cdots & R_{x x}(-p) \\
R_{x x}(1) & R_{x x}(0) & \cdots & R_{x x}(-(p-1)) \\
\vdots & \vdots & \ddots & \vdots \\
R_{x x}(p) & R_{x x}(p-1) & \cdots & R_{x x}(0)
\end{array}\right]\left[\begin{array}{c}
1 \\
a_{1} \\
\vdots \\
a_{p}
\end{array}\right]=\left[\begin{array}{c}
\sigma^{2} \\
0 \\
\vdots \\
0
\end{array}\right]
$$

Using this form, the AR parameters and $\sigma$ are determined by solving (3.22) with $p+1$ estimated autocorrelation estimates, $R_{x x}(0), \cdots, R_{x x}(p)$, and using $R_{x x}(-m)=$ $R_{x x}^{*}(m)$.

An efficient method for solution of (3.22) is the Levinson-Durbin algorithm [42]. The algorithm is recursive and provides estimates of a number, $p$, AR parameter sets for the selected model order $p$ and includes all the lower model order parameter sets. The final set at order $p$ is the solution, with the full complement of parameter sets given by $\left\{a_{11}, \sigma_{1}^{2}\right\},\left\{a_{21}, a_{22}, \sigma_{2}^{2}\right\}, \cdots,\left\{a_{p 1}, a_{p 2}, \cdots, a_{p p}, \sigma_{p}^{2}\right\}$, where the double subscript denotes model order and coefficient number, respectively. With this notation, the algorithm is initialized by,

$$
\begin{aligned}
& a_{11}=-R_{x x}(1) / R_{x x}(0) \\
& \sigma_{1}^{2}=\left(1-\left|a_{11}\right|^{2}\right) R_{x x}(0)
\end{aligned}
$$

and the recursion for $k=2,3, \cdots, p$ is given by

$$
\begin{aligned}
a_{k k} & =-\left[R_{x x}(k)+\sum_{l=1}^{k-1} a_{k-1, l} R_{x x}(k-1)\right] / \sigma_{k-1}^{2} \\
a_{k i} & =a_{k-1, i}+a_{k k} a_{k-1, k-i}^{*} \\
\sigma_{k}^{2} & =\left(1-\left|a_{k k}\right|^{2}\right) \sigma_{k-1}^{2} .
\end{aligned}
$$

Writing (3.17) in terms of the autocorrelation function expressed in terms of the YuleWalker equations and making use of the Levinson-Durbin algorithm to extend the 
summation interval is the basis for high-resolution of the AR estimator,

$$
\mathcal{P}_{A R}=\frac{\sigma^{2} T}{\left|1+\sum_{k=1}^{p} a_{k} \exp (-i 2 \pi f k T)\right|^{2}}=\Delta t \sum_{k=-\infty}^{\infty} R_{x x}[k] \exp (-i 2 \pi f k T),
$$

where

$$
R_{x x}[n]=\left\{\begin{array}{l}
R_{x x}[n], \quad \text { for }|n| \leq p \\
-\sum_{k=1}^{p} a_{p k} R_{x x}[n-k], \quad \text { for }|n|>p .
\end{array}\right.
$$

This compares to the correlogram method of classical spectral estimation, where the summation is over a finite number of autocorrelation lags estimated from the finite data length [49]. The effect of the finite sum of the classical estimator is a broadening of the main peak of the estimate, as well as the presence of sidelobes depending how the data is truncated at the endpoints. This combination of sidelobes and peak broadening for the classical estimator limit the frequency resolution for short aperture data. The AR spectral estimator is not limited by windowing effects on the data and resolution is dependent on model order selection and data aperture.

The solution of the AR model parameters can be recast in terms of the theory of linear prediction. Inspection of equation (3.18) shows it to be the equation of linear prediction where $\hat{x}$ is to be predicted given $p$ previous samples of the signal [42].

$$
\hat{x}=-\sum_{k=1}^{p} \alpha_{k} x_{n-k}
$$

In this case, the best linear prediction for $\hat{x}$ will minimize the prediction error power, $Q_{p}$, where

$$
Q_{p}=\mathcal{E}\left[\left|x_{n}-\hat{x}_{n}\right|^{2}\right]
$$

It can be shown that $\alpha_{k}=a_{p k}$ for $k=1, \cdots, p$ and $Q_{p_{m i n}}=\sigma_{p}^{2}$ so the best linear predictor for $\hat{x}$ is,

$$
\hat{x}=-\sum_{k=1}^{p} a_{p k} x_{n-k} .
$$

The above states that the parameters $\left\{a_{k 1}, a_{k 2}, \cdots, a_{k k}\right\}$, (where, as before, the sub- 
scripts $k$ and $p$ refer to the order and index) along with $\sigma_{k}^{2}$, represent the $k t h$-order linear predictor and minimum prediction error power and are identical to AR parameter estimation allowing the theory for one to apply to the other. Thus, linear prediction theory can be used for estimating AR parameters. This is very powerful in that it allows both forward and backward prediction schemes to be used in estimating AR parameters. Writing the $p t h-o r d e r$ forward prediction error $\sigma_{p n}^{2}$ as $e_{p n}$ given by,

$$
\begin{aligned}
e_{p n} & =x_{n}+\sum_{k=1}^{p} a_{p k} x_{n-k} \\
& =x_{n}+\sum_{k=1}^{p-1}\left(a_{p-1, k}+a_{p p} a_{p-1, p-k}^{*}\right) x_{n-k}+a_{p p} x_{n-p} \\
& =e_{p-1, n}+a_{p p} b_{p-1, n-1}
\end{aligned}
$$

where

$$
b_{p n}=x_{n-p}+\sum_{k=1}^{p} a_{p k}^{*} x_{n-p+k},
$$

is the backward prediction error, and the term $x_{n-p}$ is predicted from the samples $x_{n-p+1}, \cdots, x_{n}$, it can be shown that the backward prediction coefficients, $b_{p n}$, are complex conjugates of the forward prediction coefficients, $a_{p n}$ [49].

Having established the relationship between AR parameters and the forward and backward prediction coefficients, a method for estimation was chosen that combines the forward and backward linear prediction algorithms. By combining the error statistics of both the forward and backward predictors, more error points are generated resulting in an improved estimate of the AR parameters. The method of solution is that of Marple [49] and is a forward and backward least squares approach referred to as the modified covariance method.

Writing the backward prediction error, $b_{p n}$, of equation (3.31) as the vector, $\mathbf{e}_{p}^{b}$, 
and the forward error, $e_{p n}$, as, $\mathbf{e}_{p}^{f}$, yields the two $(N-p)$ length vectors given by,

$$
\mathbf{e}_{p}^{f}=\left(\begin{array}{c}
e_{p}^{f}[p+1] \\
\vdots \\
e_{p}^{f}[N]
\end{array}\right) \quad, \quad \mathbf{e}_{p}^{b}=\left(\begin{array}{c}
e_{p}^{b}[p+1] \\
\vdots \\
e_{p}^{b}[N]
\end{array}\right)
$$

where the double subscript has been replaced by a single subscript for the order and sample number indices are given explicitly. The combined error can be expressed as the length $2(N-1)$ vector, $\mathbf{e}_{p}$,

$$
\mathbf{e}_{p}=\left(\begin{array}{c}
e_{p}^{f} \\
e_{p}^{b *}
\end{array}\right)=\left(\begin{array}{c}
T_{p} \\
T_{P}^{*} J
\end{array}\right)\left(\begin{array}{c}
1 \\
a_{p}^{f b}
\end{array}\right) .
$$

Here $\mathbf{T}_{p}$ is a rectangular $(N-p) \mathrm{x}(p+1)$ data matrix given by,

$$
\mathbf{T}_{p}=\left(\begin{array}{ccc}
x[p+1] & \cdots & x[1] \\
\vdots & \ddots & \vdots \\
x[N-p] & & x[p+1] \\
\vdots & \ddots & \vdots \\
x[N] & \cdots & x[N-p]
\end{array}\right)
$$

and $\mathbf{J}$ is the $(p+1) \times(p+1)$ reflection exchange matrix given by,

$$
\mathbf{J}=\left(\begin{array}{cccc}
0 & \cdots & 0 & 1 \\
0 & \cdots & 1 & 0 \\
\vdots & \cdots & \vdots & \vdots \\
1 & \cdots & 0 & 0
\end{array}\right)
$$


The linear prediction coefficient vector $\mathbf{a}_{p}^{f b}$ is given by,

$$
\mathbf{a}_{p}^{f b}=\left(\begin{array}{c}
a_{p}[1] \\
\vdots \\
a_{p}[p]
\end{array}\right)
$$

Using the above, a least squares approach is sought which minimizes the average of the forward and backward linear prediction squared errors over the available data,

$$
\rho_{p}^{f b}=\frac{1}{2}\left[\sum_{n=p+1}^{N}\left|e_{p}^{f}[n]\right|^{2}+\sum_{n=p+1}^{N}\left|e_{p}^{b}[n]\right|^{2}\right]=\frac{1}{2} \mathbf{e}_{p}^{H} \mathbf{e}_{p}=\frac{1}{2}\left[\left(\mathbf{e}_{p}^{f}\right)^{H} \mathbf{e}_{p}^{f}+\left(\mathbf{e}_{p}^{b}\right)^{H} \mathbf{e}_{p}^{b}\right] .
$$

This leads to a set of normal equations to be solved as given by

$$
\mathbf{R}_{p} \mathbf{a}_{p}^{f b}=\left(\begin{array}{c}
2 \rho_{p}^{f b} \\
\mathbf{0}_{p}
\end{array}\right)
$$

with,

$$
\mathbf{R}_{p}=\left(\begin{array}{c}
T_{p} \\
T_{p}^{*} J
\end{array}\right)^{H}\left(\begin{array}{c}
T_{p} \\
T_{p}^{*} J
\end{array}\right)=T_{p}^{H} T_{p}+J T_{p}^{T} T_{p}^{*} J
$$

and $\mathbf{0}_{p}$ a p-element zero vector. The individual elements of $\mathbf{R}_{p}$ are given by,

$$
r_{p}[i, j]=\sum_{n=p+1}^{N}\left(x^{*}[n-1] x[n-j]+x[n-p+1] x^{*}[n-p+j]\right), \quad \text { for } 0 \leq j, k \leq p .
$$

Marple [49] describes an efficient algorithm for the solution of (3.38) with a couple of conditions. One requirement is that for $\mathbf{R}_{p}$ to be non-singular, $p \leq 2 N / 3$, i.e., the order selected can be no larger then $2 / 3$ the data length. Another caveat is that for a purely harmonic signal the recursion of the Levinson-Durbin algorithm will terminate for any $k$ where $\left|a_{k k}\right|=1$ since $\sigma_{k}=0$. Thus, for signals comprised of pure or near sinusoids, the recursion algorithm may terminate before reaching the specified model order 
The modified-covariance AR algorithm will be implemented on a signal with noise for estimating discrete frequency components and compared with the highresolution estimation results of Rajan and Bhatta [65] and the CR bound of Rife and Boorstyn [69]. It will then be used to estimate wavenumbers of the propagating modes for shallow water acoustic fields.

\subsection{Performance of Modified Covariance AR fre- quency estimator}

In order to judge the performance characteristic of the AR algorithm to estimate wavenumbers, several tests were performed on time series data for a signal plus noise. These tests were adopted from the paper of Rajan and Bhatta [65] for comparison with their results. The time domain signals were chosen with frequency and timesampling intervals that are typical of wavenumber differences and range-sampling intervals for acoustic measurements made in shallow-water waveguides. The signal data for the numerical studies were generated using

$$
y[n]=\sum_{i=1}^{L} A_{i} \exp \left[i 2 \pi f_{i} n T\right]+w[n], \quad n-0, \cdots, N-1,
$$

where $A_{i}$ are the amplitudes, $f_{i}$ are the frequencies, $T$ is the sampling interval in seconds, and $w[n]$ is the added noise assumed to be zero-mean white Gaussian. For all studies, the amplitudes were assumed equal with a value of 1 , and the sampling interval was 1 second.

\subsubsection{Frequency Estimation in the Presence of Noise}

The AR algorithm was used to estimate the frequency content of complex sinusoids with added noise. Frequency was determined by the peak location of the estimated signal spectrum. For the single frequency case, the sum in (3.41) reduces to a single 


\begin{tabular}{|c|c|c|c|c|}
\hline SNR (dB) & MUSIC & ESPRIT & AR & C-R \\
\hline \hline 5 & -51.06 & -51.78 & -58.14 & -58.16 \\
\hline 10 & -56.56 & -58.10 & -63.66 & -63.16 \\
\hline 20 & -66.52 & -68.50 & -73.94 & -73.16 \\
\hline 30 & -76.38 & -78.54 & -82.82 & -83.16 \\
\hline 40 & -86.20 & -88.56 & -90.86 & -93.16 \\
\hline
\end{tabular}

Table 3.1: SNR (dB) vs $10 \log _{10}$ (mse) for estimation of frequency $=2.3 / 2 \pi \mathrm{Hz}$.

term and the spectrum will contain a single peak. Estimates were made for increasing noise levels as indicated by the signal to noise ratio (SNR). SNR for this study is defined as $10 \log _{10}\left(\sigma_{s}^{2} / \sigma_{n}^{2}\right)$, where $\sigma_{s}^{2}$ and $\sigma_{n}^{2}$ are the mean-square signal power and noise power, respectively. Estimates were made for a fixed data length of 40 samples $(N=40)$, and 100 realizations of the additive noise were generated to determine performance statistics. AR model order was determined by trial and error at each SNR as the maximum order that gave only a single peak for all realizations of the signal. For $S N R<30 \mathrm{~dB}$ a model order of 13 was used which is approximately equal to $N / 3$. The mean squared error of (3.5) was used as a basis for performance and compared to the $\mathrm{CR}$ bound given by (3.6). Results are given in $\mathrm{dB}$ calculated as $10 \log _{10}$ (mse). The ideal result for an unbiased estimator is one that achieves the CR bound. In addition to the CR bound, the AR results are compared with the estimates of the MUSIC (Multiple Signal Classification Method) and ESPRIT (Estimation of Signal Parameters via Rotational Invariance Techniques) determined by Rajan [65], where his results have been converted to match the scale defined for this study.

Frequency estimates were made for individual frequencies $f_{1}=2.5 / 2 \pi \mathrm{Hz}$ and $f_{2}=2.3 / 2 \pi \mathrm{Hz}$ for SNR values from 5 to $40 \mathrm{~dB}$. The mse results for estimating $f_{2}$ are shown in table 3.1. Bias was calculated for each of the two frequencies at the different SNR and are shown in table 3.2. From the results given in tables 1 and 2 the AR estimator shows the following behavior.

1.) The mean-squared error is better for the AR estimator then either MUSIC or 


\begin{tabular}{|c|c|c|}
\hline SNR $(\mathrm{dB})$ & freq $=2.5 / \pi \mathrm{Hz}$ & freq $=2.3 / \pi \mathrm{Hz}$ \\
\hline \hline 5 & & $-2.56 \times 10^{-4}$ \\
\hline 10 & & $7.88 \times 10^{-5}$ \\
\hline 20 & $-7.38 \times 10^{-5}$ & $-1.02 \times 10^{-5}$ \\
\hline 30 & & $-4.06 \times 10^{-6}$ \\
\hline 40 & $-2.39 \times 10^{-5}$ & $-3.47 \times 10-5$ \\
\hline
\end{tabular}

Table 3.2: SNR $(\mathrm{dB})$ vs bias of estimates for frequencies $f_{1}=2.5 / 2 \pi \mathrm{Hz}$ and $f_{3}=$ $2.3 / 2 \pi \mathrm{Hz}$.

ESPRIT and approaches the CR bound at all SNR levels.

2.) The bias error is of the same order or better then MUSIC and ESPRIT at all SNR levels.

Although these results are compared with the CR bound, the estimation results at each different SNR had a small bias which violates the criterion of an unbiased estimator. Given a limited number of realizations, this discrepancy can cause the estimator to perform better than the bound as the results in table 3.1 suggest at low SNR. Nevertheless, the comparision is still of use for evaluating results from different estimators. Further, the bias for SNR $\& 10 \mathrm{~dB}$ was sufficiently small that $m s e$ effectively gives a measure of the variance of the estimates. The variance of $5.3 \times 10^{-9}$ at $30 \mathrm{~dB}$ SNR also compares favorably to the estimate $2.5 \times 10^{-8}$ as determined by Sakai's [71] result which is proportional to $S N R_{\text {lin }}^{-2} / N$. Finally, it should be noted, in order to measure the small variances in the estimated frequencies at high SNR, a large number of terms was used in the summation interval of (3.25) to give sufficient discritization in the frequency domain. For SNR of $30 \mathrm{~dB}, 2^{18}$ points were used in the summation.

\subsubsection{Frequency Resolution}

The next study uses the AR estimator to identify two closely spaced frequencies in the presence of added noise. This is representative of the case of closely spaced 


\begin{tabular}{|c|c|c|}
\hline SNR $(\mathrm{dB})$ & ESPRIT & AR \\
\hline \hline 20 & -53.74 & -61.16 \\
\hline 30 & -63.66 & -70.28 \\
\hline 40 & -73.74 & -77.70 \\
\hline 50 & -83.72 & -79.48 \\
\hline
\end{tabular}

Table 3.3: SNR (dB) vs $10 \log _{10}$ (mse) for estimation of $f_{o}$ using ESPRIT and AR. $f_{o}=0.4 \mathrm{~Hz}, \delta f=0.011 \mathrm{~Hz}$.

wavenumbers for a shallow water waveguide. The signal model of (3.41) was used with two frequencies of various separation frequencies, $\delta f$, that ranged from $0.009 \mathrm{~Hz}$ to $0.027 \mathrm{~Hz}$. Using equation (3.1), at $20 \mathrm{~dB}$ SNR, a minimum model order of 13 would be required to resolve $\delta f=0.009 \mathrm{~Hz}$. The frequencies used to generate the complex signal were $f_{1}=f_{o}=0.4 \mathrm{~Hz}$ and $f_{2}=f_{o}-\delta f \mathrm{~Hz}$. Each signal was comprised of 40 samples and 100 realizations were made of the noise at each SNR. Model order was again selected by trial and error to yield two spectral peaks for all signal realizations with a fixed order of 13 for SNR under $40 \mathrm{~dB}$. Table 3.3 gives the mse estimates vs SNR for estimation of $f_{o}=0.4 \mathrm{~Hz}$ and a frequency separation of $0.011 \mathrm{~Hz}$. In these cases where both estimators were able to resolve the two frequencies, the results indicate that the AR estimator performs better than ESPRIT at all SNR for resolving closely spaced frequencies. Similar results were observed for the other estimates of closely spaced frequencies.

\subsubsection{Size of Data Vector and Model Order Selection}

In the previous studies, AR model order was selected by trial and error based on the number of known sinusoids in the signal. However, the resolution and variance of the estimator were shown by Marple [49] and Sakai [71] to be a function of total data length, model order, and SNR. Further, although the all pole model of the AR estimator suggest that model order be selected equal to the number of sinusoids in the signal, the presence of noise requires a higher model order to best fit the model to 


\begin{tabular}{|c|c|c|}
\hline Samples & $\begin{array}{c}\text { ESPRIT } \\
\text { \% correct }\end{array}$ & $\begin{array}{c}\text { AR } \\
\text { \% correct }\end{array}$ \\
\hline \hline 40 & 20 & 34 \\
\hline 60 & 21 & 55 \\
\hline 80 & 88 & 81 \\
\hline 100 & 99 & 98 \\
\hline
\end{tabular}

Table 3.4: Probability of success for estimation of $\mathrm{f}=0.4 \mathrm{~Hz}$ with $\mathrm{SNR}=10 \mathrm{~dB}$, model order $\mathrm{p}=20$, and increasing data length. Percent correct for estimating ( $\mathrm{f} 1 \pm$ 0.001) $\mathrm{Hz}$.

the data. As noise increases, the number of model parameters required to best fit the data also increases. In this section, the effect of data length is examined as it effects resolution in resolving signals comprised of closely spaced sinusoids. An additional study is then run to examine the effect of model order selection on resolution and variance and the presence of spurious peaks in the spectral estimates.

The first of these tests was conducted to identify two frequencies, $f_{1}=0.4 \mathrm{~Hz}$ and $f_{2}=0.39 \mathrm{~Hz}$, with SNR $=10 \mathrm{~dB}$ for various data lengths. Data length was increased from 40 points to 100 points, and the probability of success in identifying $f_{1} \pm 0.001$ $\mathrm{Hz}$ was determined for 100 realizations of the noisy signal. For $S N R=10 \mathrm{~dB}$ and $N=40$, a model order of 20 was necessary to theoretically resolve the two freqencies. This was used for all data lengths in the study. The results are shown in table 3.4 and compare reasonably well with the ESPRIT results of Rajan and Bhatta [65]. Overall, the AR estimator performs better then MUSIC and ESPRIT for short data lengths of 40 and 60 , and on par with them for longer data lengths.

A similar study was conducted to examine the effect of model order selection on frequency estimates. The signal was comprised of 40 samples with two sinusiods at $f_{1}=0.4 \mathrm{~Hz}$ and $f_{2}=0.35 \mathrm{~Hz}$ with an SNR of $20 \mathrm{~dB}$. Of particular interest is the effect of resolution on model order, and also the presence of spurious peaks in the spectrum for high model orders. The effect of model order selection on the spectral estimates is clearly shown in figures 3-4 and 3-5. In each of the figures, the left column shows the 

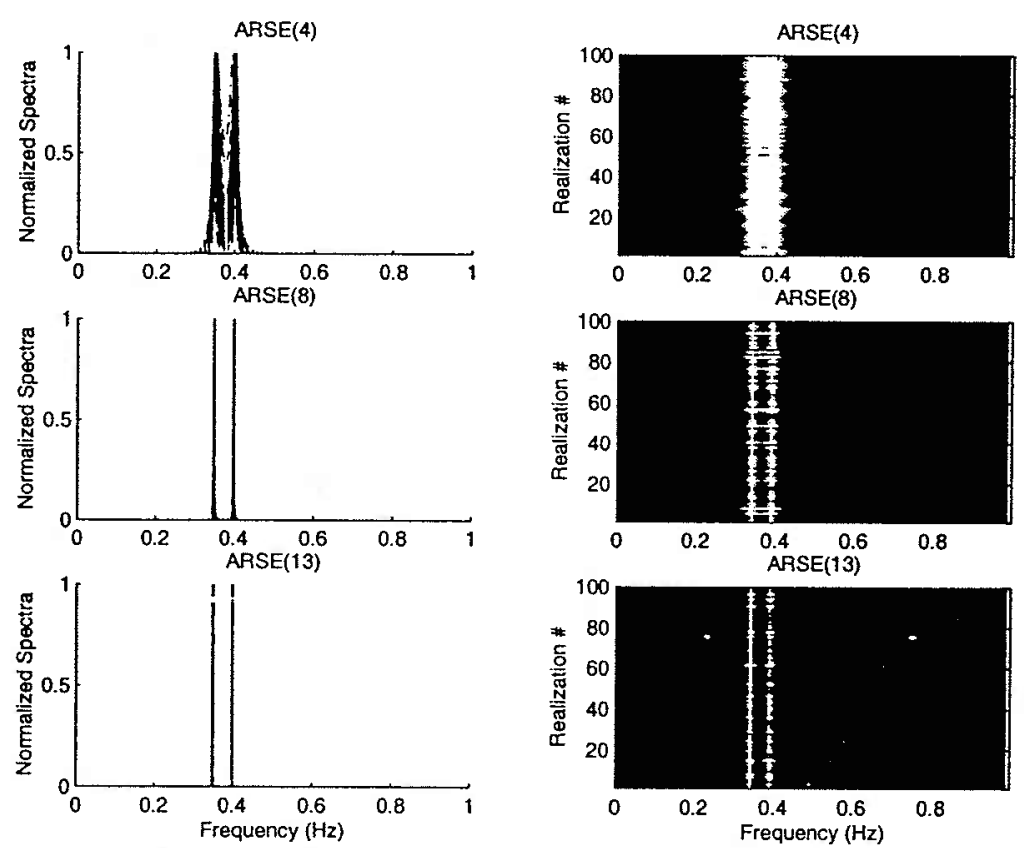

Figure 3-4: Spectral estimates for 100 realizations of signal with 2 sinusoids in noise. $\mathrm{SNR}=20 \mathrm{~dB}, f_{1}=0.4 \mathrm{~Hz}, f_{2}=0.35 \mathrm{~Hz}$. AR model order is indicated at top of each plot.

results of each spectrum for the 100 signal realizations plotted on top on one another. The right column shows the spectra for all realizations lined up next to one another with any peaks greater than $60 \mathrm{~dB}$ down indicated in white. This display gives an indication of both peak width and location. It also illustrates the stability of the peak locations for the two excited frequencies for all realizations. This type of behavior is typical of wavenumber estimates for range-independent shallow water waveguides and is used to identify the prominant propagating modes using the sliding window transform method. From the plots, it is clear that as model order increases, the peak widths decrease. However, as model order increases past $N / 3$, spurious peaks can be introduced into the spectra. The presence of extra peaks causes ambiguity in the selection of the peaks which represent the true signal. Model order selection for AR spectral estimates with no a priori knowledge must strike a balance between resolution and the introduction of spurious peaks.

Marple [49] reviews several methods and criteria put forth for determining model order selection, including the final predicion error (FPE) [1] and Akaike information 

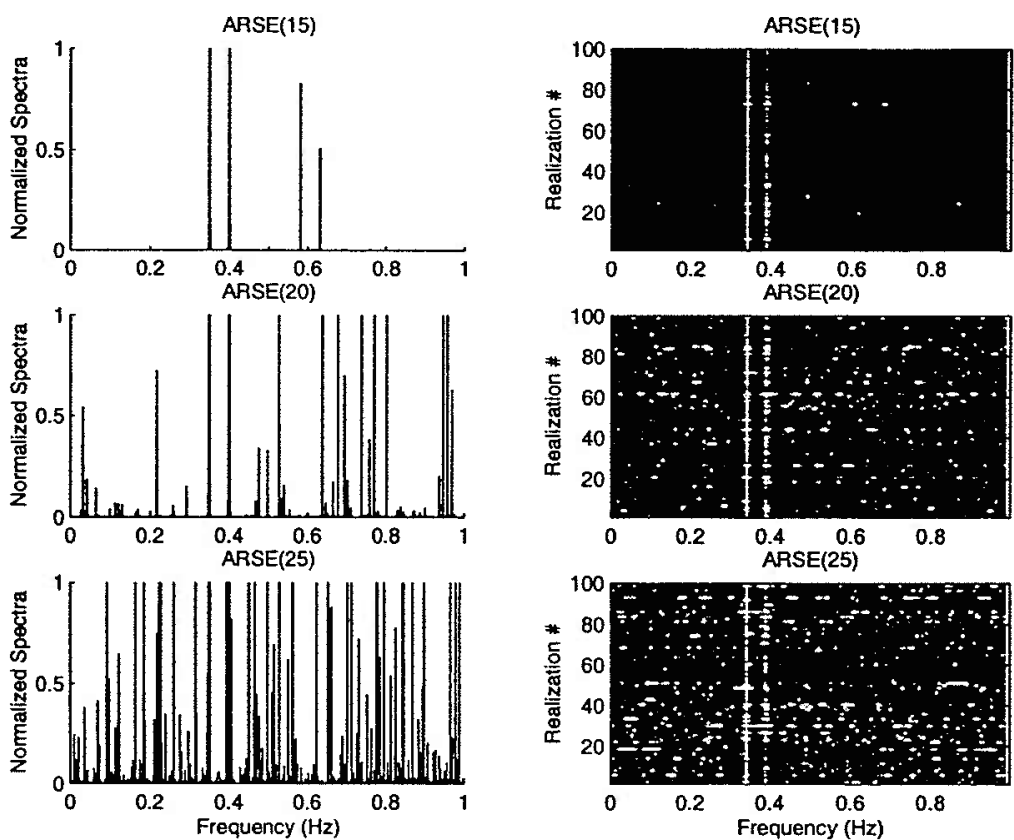

Figure 3-5: Spectral estimates for 100 realizations of signal with 2 sinusoids in noise. $\mathrm{SNR}=20 \mathrm{~dB}, f_{1}=0.4 \mathrm{~Hz}, f_{2}=0.35 \mathrm{~Hz}$. AR model order is indicated at top of each plot.

criterion (AIC) of Akaike [2], where Gaussian noise statistics are assumed. Each of these criteria seek a minimum value in the prediction error variance with a penalty applied for increased model order $\mathrm{p}$ after the minimum is reached. Marple also describes the minimum description length (MDL) and criterion autoregressive transfer (CAT) function. After examining all these criteria, Marple states that the results of applying each to real data are inconsistent. Various empirical studies are then presented which suggests that for noisy data a model order of $N / 3$ to $N / 2$ yield good results on short data segments. This hypothesis is tested using the AR estimator used for this work. For this study 40 data points were used with an SNR of $20 \mathrm{~dB}$ for the same frequencies used in the previous case, $f_{1}=0.4 \mathrm{~Hz}$ and $f_{2}=0.39 \mathrm{~Hz}$. Success rates were calculated for 100 realizations of the noise for model order of between 2 and 25 , with model order $p=13$ approximately $N / 3$. The results shown in table 3.5 confirm the empirical results cited by Marple as well as those shown in figures 3-4 and 3-5. Success rates are maximized for a model order $p=13$. Results marked by an $\mathrm{X}$, indicate that no peaks were observed in that bin for any realizations. It was ob- 


\begin{tabular}{|c|c|c|c|c|}
\hline Samples & $\mathrm{mse}(\mathrm{f} 1)$ & $\%(\mathrm{f1} \pm 0.001)$ & $\mathrm{mse}(\mathrm{f} 2)$ & $\%(\mathrm{f} 2 \pm 0.001$ \\
\hline \hline 2 & $\mathrm{X}$ & $\mathrm{X}$ & 0 & 1 \\
\hline 5 & $\mathrm{X}$ & $\mathrm{X}$ & 0 & 0 \\
\hline 10 & -57.92 & 65 & -58.98 & 71 \\
\hline 13 & -62.64 & 78 & -64.14 & 88 \\
\hline 15 & -62.48 & 83 & -63.42 & 83 \\
\hline $20^{*}$ & $-22 /-64$ & 79 & $-26 /-66$ & 89 \\
\hline 25 & -18 & 59 & -28 & 79 \\
\hline
\end{tabular}

Table 3.5: Probability of success for estimation of $f=0.4 \mathrm{~Hz}$ with SNR $=10 \mathrm{~dB}$ and increasing model order.

served that as model order was increased beyond $N / 3$, spurious peaks contaminated the spectra. However, for the case of $\mathrm{p}=20$, where two values are listed, a small number of outliers were removed using a priori information and success rates were improved. However, outlier removal requires information that may not be available so is not generally a viable approach to improving on estimates.

A final comment about model order selection is in regard to the true nature of the process being modelled by the AR parameters. For a true AR model, there exists a given model order $p$ for which the prediction error variance estimate is minimized. For model orders greater then $p$, the prediction error variance is flat and and gives a method for selecting the exact order of the AR process. However, for a non-AR process, prediction error variance is a monontonically decreasing function of $p$ and no minimum will be reached. This contributes to the problem of selecting a model order a priori for a non-AR process.

\subsubsection{Application of AR estimator to acoustic data}

It is now of interest to use the AR spectral estimator to determine the horizontal wavenumbers for the normal modes propagating in a shallow-water waveguide. Correcting for geometric spreading and adding a noise term, the signal to be analyzed 


\begin{tabular}{|c|c|c|c|}
\hline Depth $(\mathrm{m})$ & $c_{s}(\mathrm{~m} / \mathrm{s})$ & $\rho\left(\mathrm{g} / \mathrm{cm}^{3}\right)$ & $\alpha($ nepers $/ \mathrm{m})$ \\
\hline \hline $0-40$ & 1515.0 & 1.0 & 0.0 \\
\hline $40+$ & 1800.0 & 1.56 & 0.001 \\
\hline
\end{tabular}

Table 3.6: Pekeris ocean model. Frequency $=100 \mathrm{~Hz}$, source depth $=20.5 \mathrm{~m}$ and receiver depth $=15.5 \mathrm{~m}$.

\begin{tabular}{|c|c|c|c|}
\hline Mode No. & $k_{n}(1 / \mathrm{m})$ & $\Psi_{s}$ & $\Psi_{r}$ \\
\hline \hline 1 & 0.409260 & 0.179 & 0.204 \\
\hline 2 & 0.392023 & 0.181 & 0.074 \\
\hline 3 & 0.361364 & -0.003 & -0.178 \\
\hline
\end{tabular}

Table 3.7: Modal eigenvalues and corresponding amplitudes at source and receiver for Pekeris ocean model.

can be represented in terms of the acoustic pressure, $P(n \Delta r)$, by [65]

$$
y[n]=\sqrt{n \Delta r} P[n \Delta r]+w[n \Delta r]=\sum_{l=1}^{L} A_{l} \Psi_{l}+w[n \Delta r]
$$

where the range interval is sampled by $\Delta r$, and $\Psi_{l}=e^{i k_{l} n \Delta r}$, is the mode function with horizontal wavenumber $k_{l}$ and amplitude $A_{l}$. The objective is to estimate the $k_{l}$ from the data measured along $r$. To generate data, KRAKEN [59], a normal-mode acoustic propagation code, was used. The output of KRAKEN gives complex pressure fields as a function of range as well as eigenvalue information for the input environment and source frequency. The environment model used was a Pekeris ocean model with parameters listed in table 3.6. The source frequency was $100 \mathrm{~Hz}$, with source and receiver at depths of $20.5 \mathrm{~m}$ and $15.5 \mathrm{~m}$, respectively. The field was sampled on a $1 \mathrm{~m}$ range grid. Mode numbers and modal amplitudes at the source and the receiver are listed in table 3.7. The KRAKEN results show that mode 3 is only weakly excited at the source depth and will be difficult to estimate. For the given field, wavenumber estimates are made and mse calculated for various aperture lengths and SNR levels. 


\begin{tabular}{|c|c|c|c|c|}
\hline SNR (dB) & Aperture $(\mathrm{m})$ & Mode No. & ESPRIT & AR \\
\hline \hline 40 & 240 & 1 & -81.32 & -83.00 \\
& & 2 & -69.74 & -72.46 \\
& & 3 & -19.38 & -39.68 \\
\hline 40 & 360 & 1 & -88.61 & -83.00 \\
& & 2 & -77.58 & -78.70 \\
& & 3 & -53.84 & -61.62 \\
\hline \hline 20 & 360 & 1 & -69.40 & -73.98 \\
& & 2 & -61.76 & -66.82 \\
\hline 20 & 480 & 1 & -81.14 & -79.50 \\
& & 2 & -71.62 & -71.48 \\
\hline
\end{tabular}

Table 3.8: SNR (dB) vs $10 \log _{10}$ (mse) of eigenvalues for different data aperture lengths.

AR model order was selected as $\mathrm{N} / 3$ as determined by total aperture length. Table 3.8 shows the results of these calculations. The results, as indicated by small $m s e$, show that the strong modes are detected for all aperture lengths and SNR levels. The weak third mode is identified with confidence only for high SNR and long aperture. Consistent with the previous study of time series data, the AR estimator performs as well as or slightly better than the other high-resolution methods.

\subsection{Wavenumber Estimates for Range-Dependent Synthetic Acoustic Data}

Rajan and Bhatta [65] also studied wavenumber estimation using high-resolution estimators for range-dependent acoustic environments. Their method examined wavenumber estimates resulting from short aperture transforms using data from a model that included bathymetry with a constant slope in range. The bathymetric slope was 1.67 $\mathrm{m} / \mathrm{km}$, which equates to an angle of $0.955^{\circ}$. In general, their wavenumber estimators yielded wavenumber values that were between those expected for the deepest 
and shallowest portion of the aperture. They concluded that errors associated with wavenumber estimates under the assumption of locally range-independent segments are small. Further, given the previous studies, the errors associated with this slight range dependence would be indistinguishable in the presence of noise. The behavior described by Rajan and Bhatta was observed for wavenumber estimates made on an independent data set provided as part of a recent inversion techniques workshop (ITW) [40].

\subsubsection{Inversion Techniques Workshop}

The ITW was conducted in order to evaluate the current state of the art in geoacoustic inversion algorithms for application in range-dependent shallow-water waveguides. Synthetic acoustic pressure field data were generated for several test case environments and given to participants for analysis. The data were provided at a number of discrete frequencies on both horizontal and vertical arrays. Properties of the water column were given, as were source and receiver depths. Bathymetry was assumed known to within a meter. No information was given regarding the sediment properties, and participants were asked to invert for compressional wave speed, attenuation, and density in the bottom. A total of four different synthetic tests cases (TC0-TC3) were given for analysis. TC0 was a calibration case, shown in figure 3-6, and was characterized by a constant bathymetric slope where the stratigraphy in the bottom followed the bathymetry. The input model for TC0 was given so that participants could calibrate their forward propagation models to the given synthetic data. TC0 was used in this work to examine the characteristic of the AR wavenumber estimator for a range-dependent problem. Because the model was given, KRAKEN could be used to calculate the range-dependence of the eigenvalues. Sliding window AR estimates could be made and wavenumber estimates compared to the expected values at a given range step. The sliding window estimator results are plotted in figure 3-7 for a $1000 \mathrm{~m}$ aperture with a step length of $50 \mathrm{~m}$. The data was provided on a 5 meter 


\section{TC0 - Calibration}

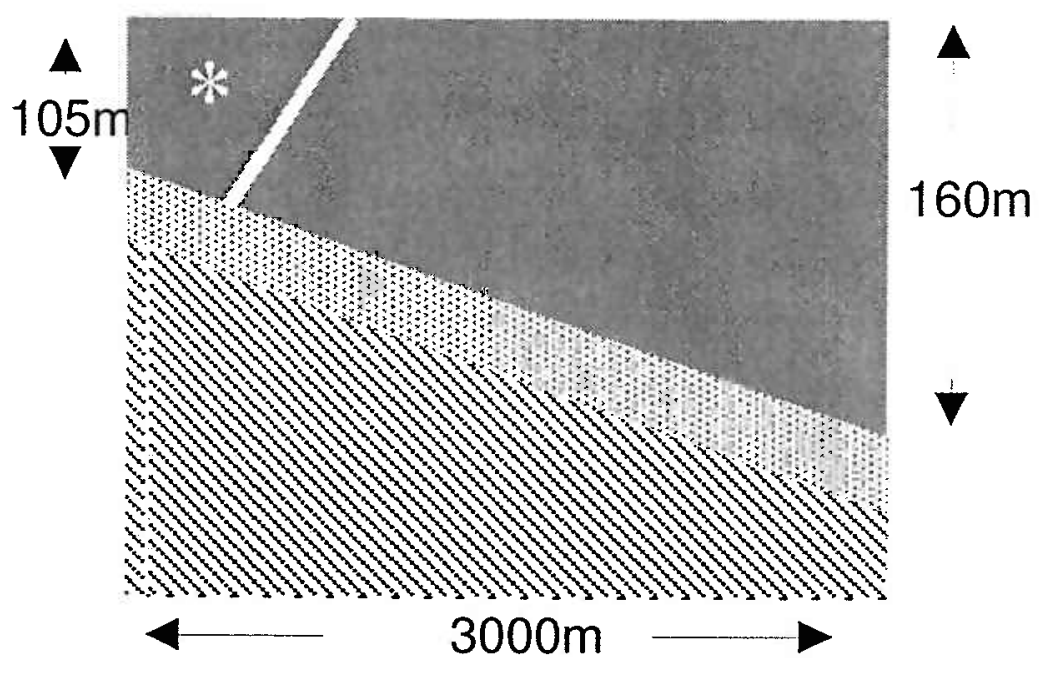

Figure 3-6: Environment model for Inversion Workshop TC0.

range grid resulting in an N/3 model order selection of 66 for a $1000 \mathrm{~m}$ aperture. The estimates for the $50 \mathrm{~Hz}$ data and the true values are shown in figure 3-8. The true values are bounded by the values determined for the endpoints of the $1000 \mathrm{~m}$ aperture and represent the minimum and maximum values within the aperture. Similar to the Rajan study, the AR estimated wavenumbers fall between the minimum and maximum values for most range intervals. And, although the resulting wavenumber values did not depart very much from expected values, the inconsistent prediction behavior, shown as oscillations about the true values, is undesirable. One can conclude from this study that the locally range-independent assumption cannot be applied for constant slopes in bathymetry of even 1 degree, and that bathymetry must be accounted for in the wavenumber estimates. Harrison and Siderius [37] suggest a method for using range-independent models to calculate range-dependent effects through the use of an effective geometry. In their work, an environment similar to TC0 is considered and some of their ideas may be applicable to determining wavenumbers for the continuously varying waveguide. Their ideas are similar in nature to those of Fernandez [24], where horizontal wavenumber analysis is investigated for range-dependent environ- 


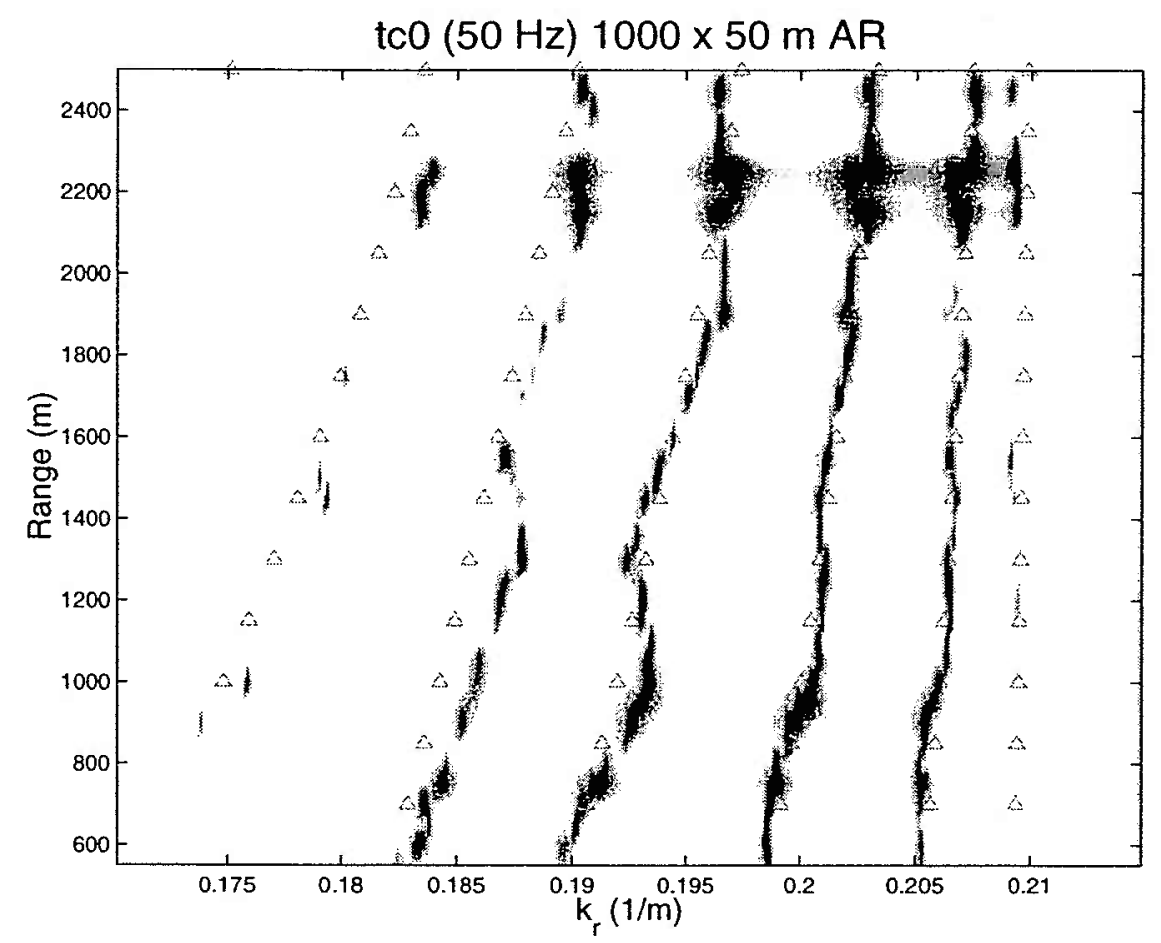

Figure 3-7: Sliding Window spectral estimates for $50 \mathrm{~Hz}$ TC0. Triangles are the range-dependent horizontal wavenumbers determined using KRAKEN plotted over the wavenumber estimates.

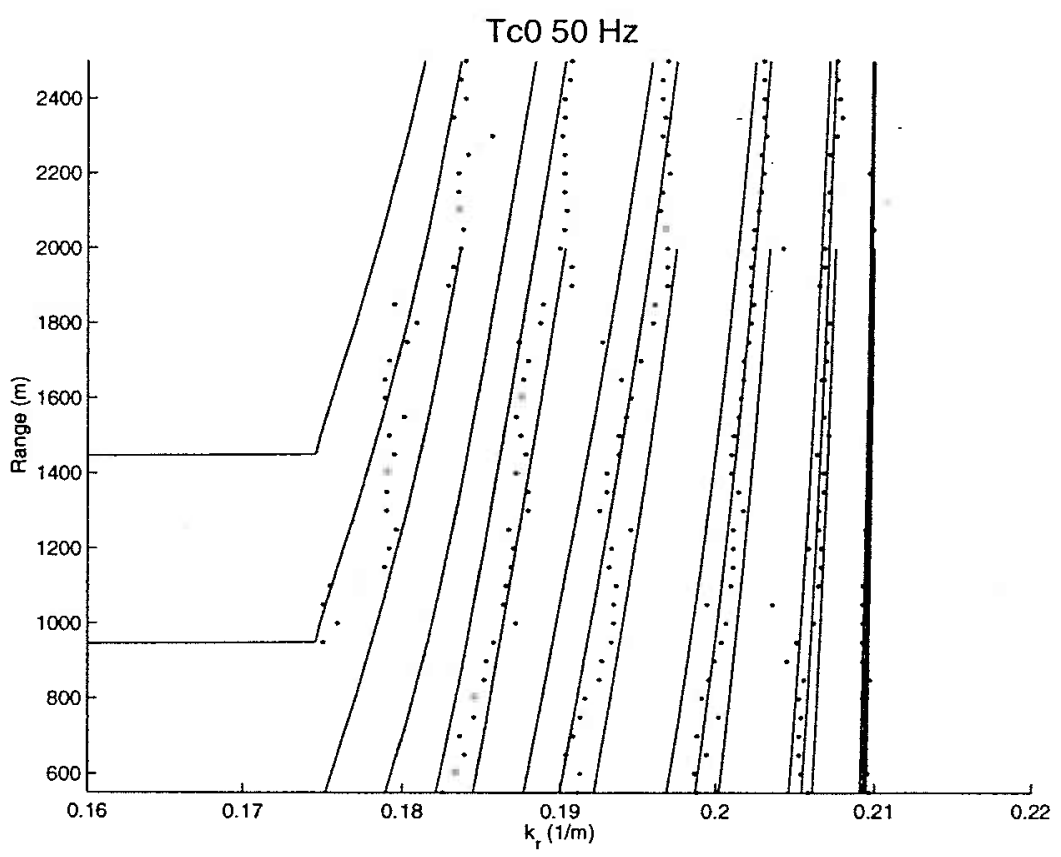

Figure 3-8: Wavenumber estimates for TC0 (dots) plotted with values for the starting range, center range, and end range of the transform aperture. 


\section{TC2 - Shelfbreak}

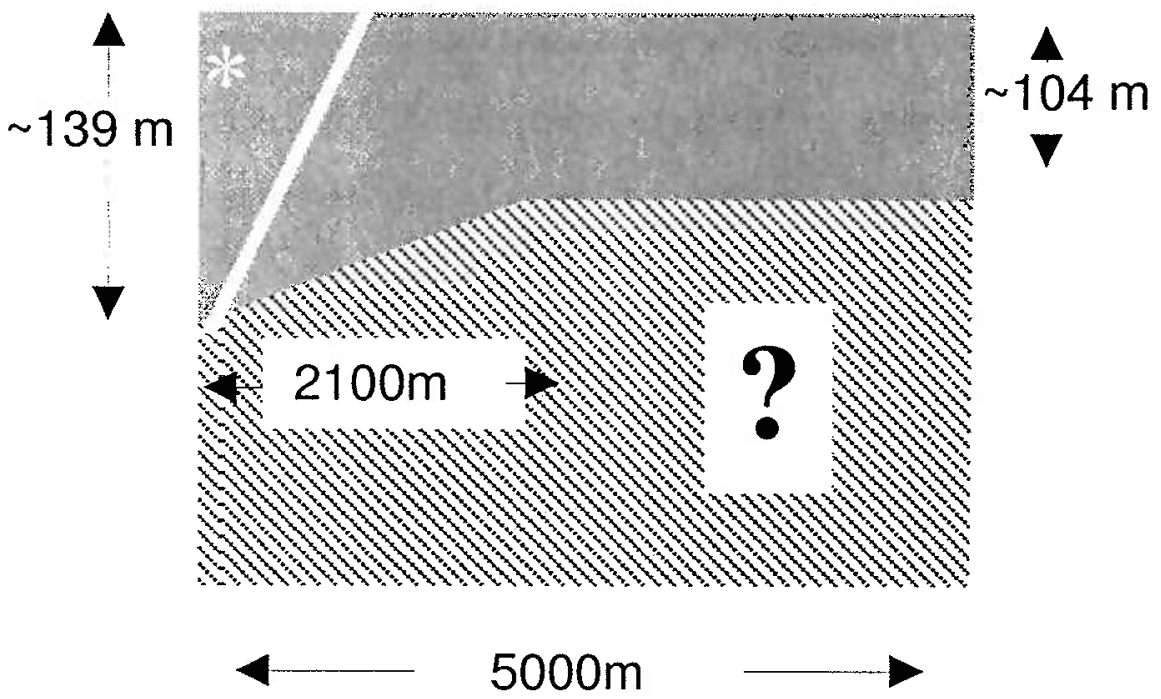

Figure 3-9: Shelfbreak environmental model for Inversion Workshop TC2.

ments by migrating the measured field to an effective range grid to approximate a range-independent environment. Another area of investigation for wavenumber analysis in range-dependent environments would be the application of time-varying autoregressive estimators (TVAR) [43] [82]. The TVAR model allows for time-variation in the AR model coefficients for non-stationary time series data that can include both trends and discrete changes in the signal. However, as the AR estimator discussed here does not perform well for the continuously varying environment, TC0 and TC1 (both constant slope) were not analyzed for the workshop. Instead, emphasis was placed on examining wavenumber estimates for range-dependent cases where bathymetric effects are minimized, this includes regions of slightly undulating bathymetry, and regions of constant local bathymetry. An example of the later is TC2, shown in figure 3-9, where a flat bottom occurs after a shelf-break. The wavenumber estimates at $50 \mathrm{~Hz}$ for TC2 are shown in figure 3-10 and appear to be range-independent for ranges after the break. Workshop organizers confirmed this to be true. In this case, the data again was sampled on a 5 meter grid, and a $1000 \mathrm{~m}$ sliding window estimator was used with a step size of $50 \mathrm{~m}$ and a model order of 20 . The low model order 
was used because the data is noise free and approximates a sum of pure sinusoids after the shelf break causing the Levinson-Durbin algorithm to terminate at a low model order. The effect of the low model order in the range-dependent region is low resolution as shown in the figure. However, if model order is selected high enough in the range-dependent region, results similar to those of TC0 could be expected. By using an algorithm with an adaptive model order selector as the window stepped through range, better resolution of the modes in the range-dependent region could be obtained as shown in figure 3-11. For this result, model order was linearly decreased from $\mathrm{p}=66$ to $\mathrm{p}=20$ as the window began to overlap the range-independent region. This result highlights the effect of model order selection on the AR estimates as discussed previously. Additionally, sliding window wavenumber estimates were applied to the TC2 data with $40 \mathrm{~dB}$ additive white noise added. In this case, model order was fixed at 66 and a $1000 \mathrm{~m}$ sliding window aperture used. The results are similar to the results for the adaptive estimator with no noise. The effect of the noise on the estimates were minimal, but allowed the recursion in the AR algorithm to finish at all ranges eliminating the requirement for adaptive model order selection. 


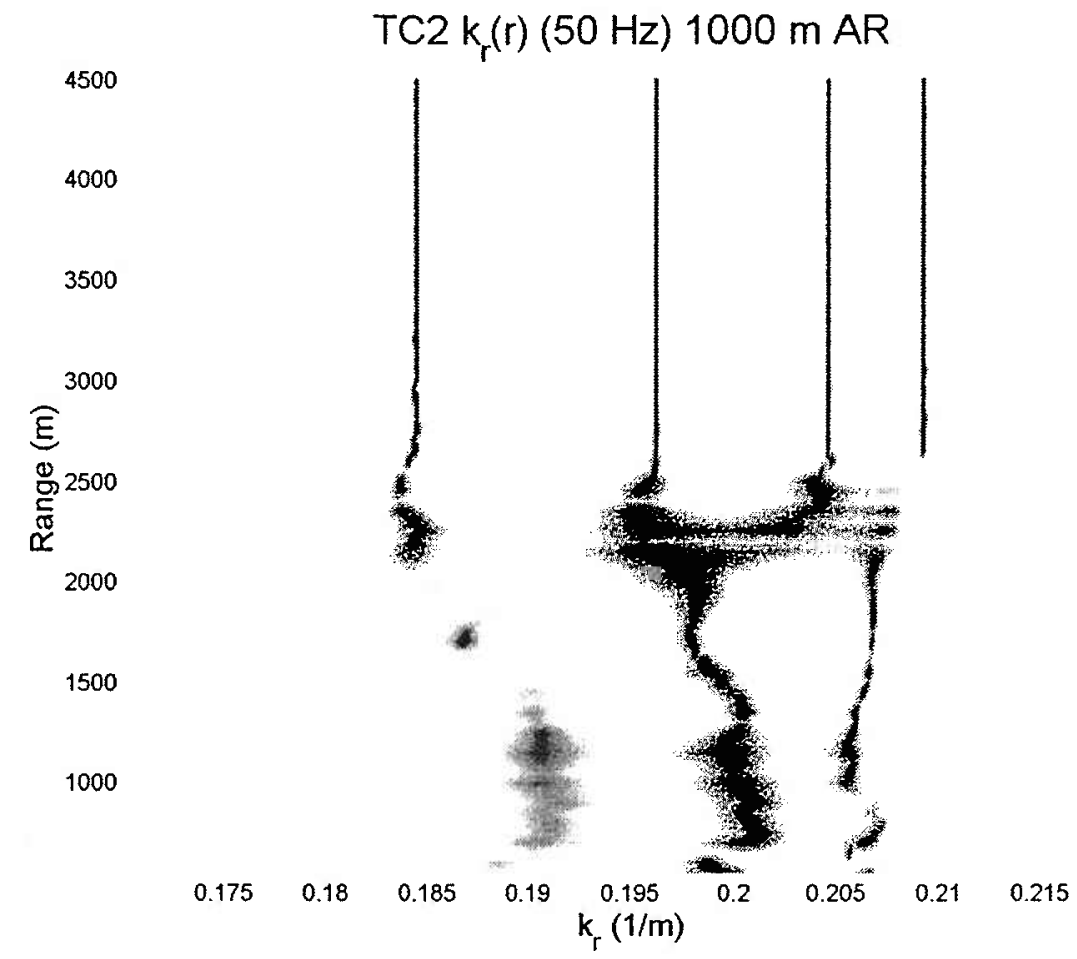

Figure 3-10: Wavenumber estimates showing range-independent region after the shelfbreak for Inversion Workshop TC2.

TC2 $k_{r}(r)(50 \mathrm{~Hz}) 1000 \mathrm{~m} \mathrm{AR}$

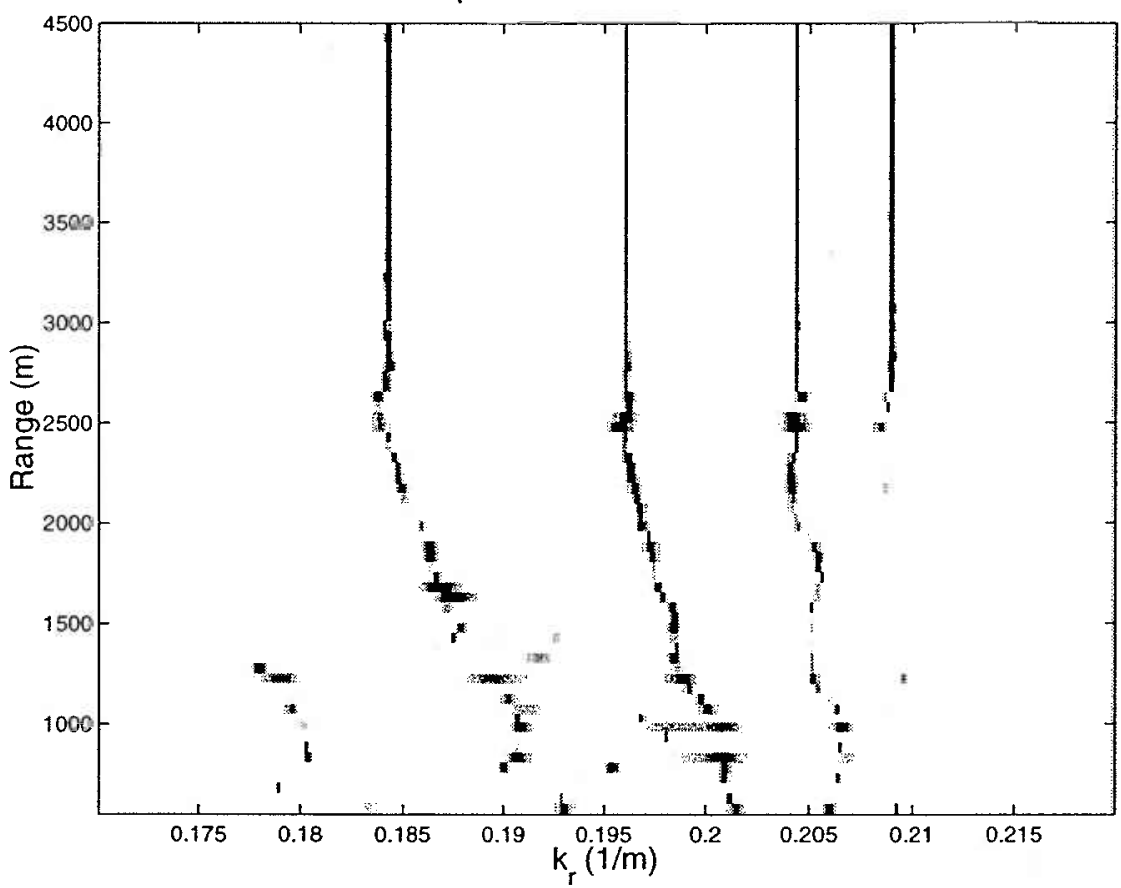

Figure 3-11: Wavenumber estimates using adaptive AR model order selector showing wavenumber evolution for Inversion Workshop TC2. 


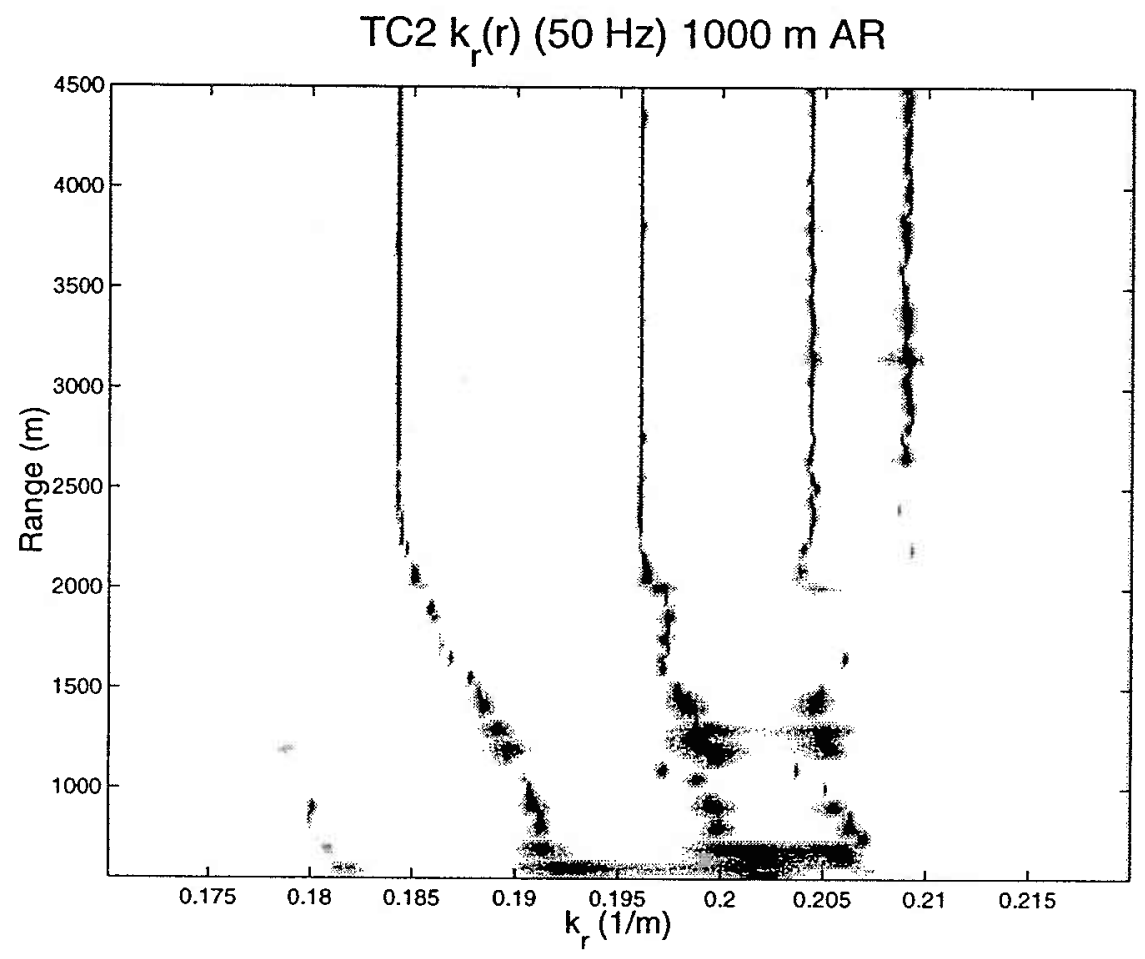

Figure 3-12: Wavenumber estimates for order 66 AR estimator showing wavenumber evolution Inversion Workshop TC2 data with $60 \mathrm{~dB}$ noise added. 


\section{TC3 - Flat Bottom}
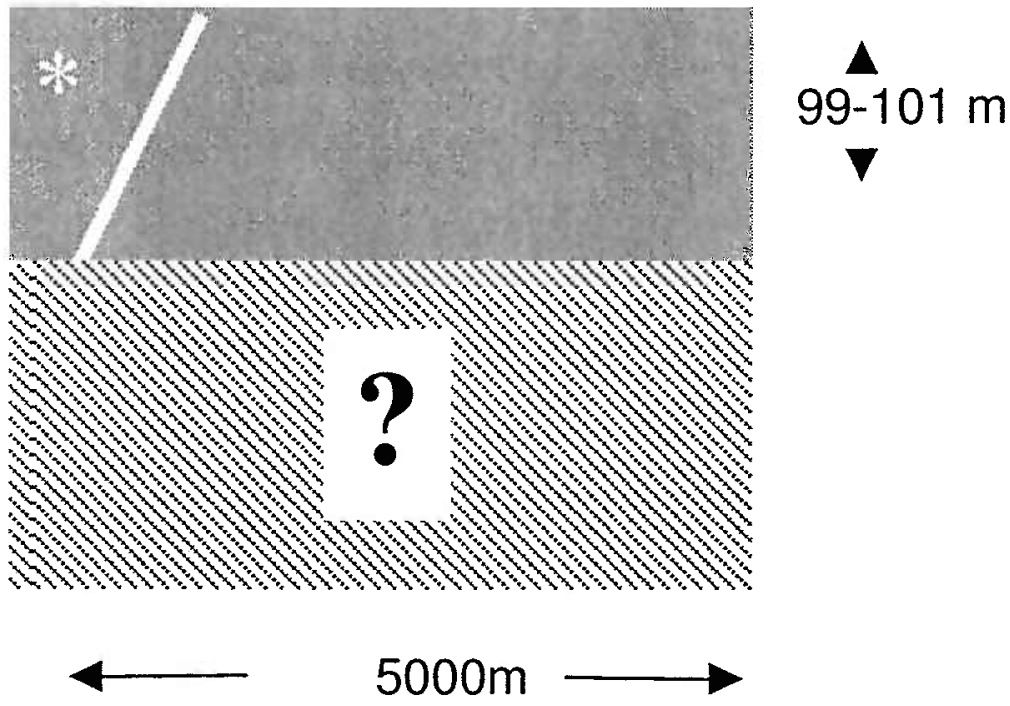

Figure 3-13: Environment model for Inversion Workshop TC3.

\subsubsection{Range-Dependent Sediment - TC3}

A particularly interesting data set for analysis was TC3 where the bathymetry was flat and range-dependence occurred solely in the sediment. The geometry and other known parameters are shown in figure 3-13, along with $50 \mathrm{~Hz}$ data on a 5 meter range grid for the upper receiver in figure 3-1. No noise was added to the data. The only a priori information given about the bottom is that "there is an intrusion in the sediment" [40]. No other information about the intrusion is given. This point is important because depending on the inversion algorithm used, assumptions may have to be made about the nature of the intrusion. Features such as location and shape may have to be assumed [13] that have an impact on the inversion results. Techniques based on wavenumber estimates need no such assumption and are only limited by their ability to resolve features using short data apertures.

Using the sliding window AR estimator on the $50 \mathrm{~Hz}$ data with a model order of 30 yields the wavenumber-range plot shown in figure 3-14. From the figure two range-independent regions can be identified directly by the constant wavenumber values. The other regions of low resolution are a result of the fixed model order 
TC3 $k_{r}(r)(50 \mathrm{~Hz}) 1000 \mathrm{~m} \mathrm{AR}$

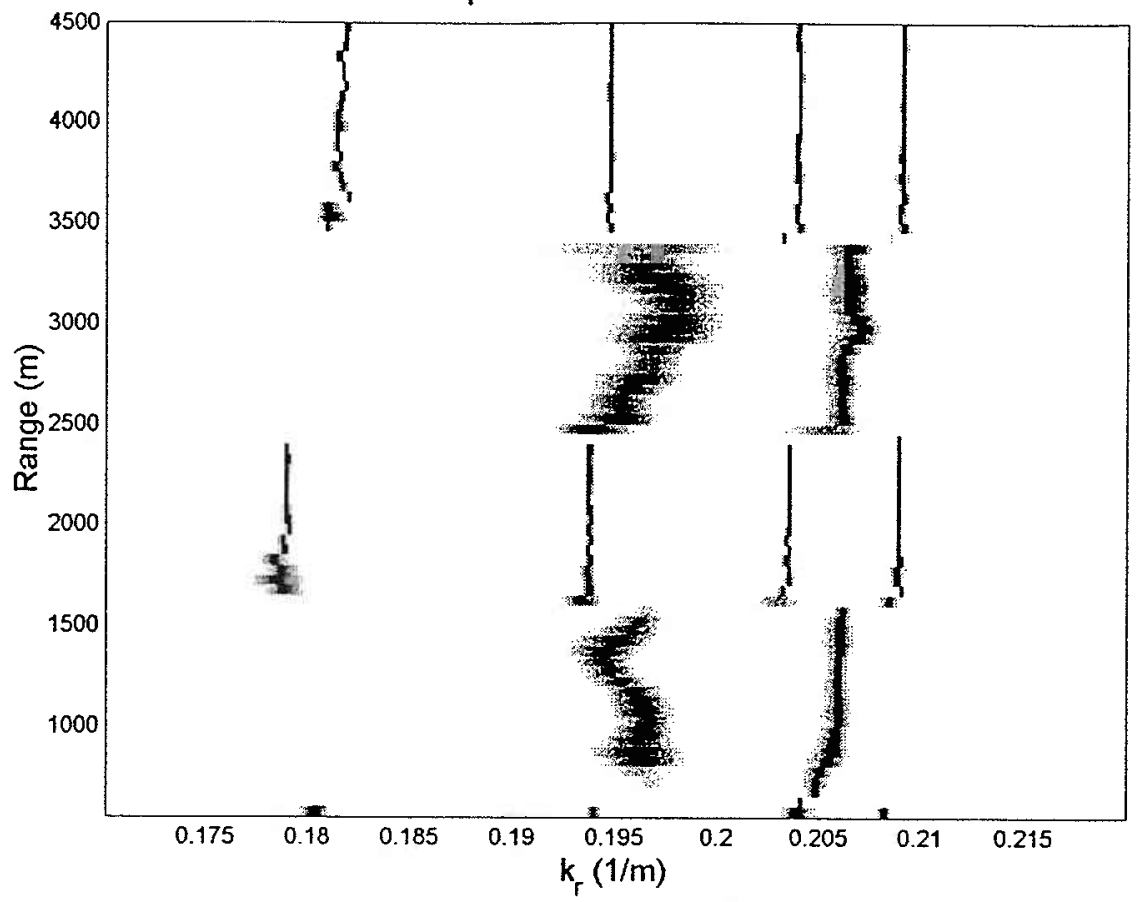

Figure 3-14: Wavenumber estimates for range-dependent sediment of Inversion Workshop TC3.

used with the sliding window estimator. In those regions, the window is overlapping two range-independent regions with with wavenumber values very closely spaced. Examining the range-independent regions of the figure, and recalling that the range axis represents ranges at the center of the aperture, it is possible to determine the range of data included in each aperture. Thus, the result at range $=1100 \mathrm{~m}$ includes data that spans the range interval $600 \mathrm{~m}<\mathrm{R}<1600 \mathrm{~m}$, and results at range $=2100$ $\mathrm{m}$ includes data that spans $1600 \mathrm{~m}<\mathrm{R}<2600 \mathrm{~m}$. By including all the data spanned by the aperture as the window slides, the range-independent regions identified from figure 3-14 can be extended by 500 meters at each end to clearly demarcate the range-independent regions for TC3 as shown in figure 3-15. Using this method, it was determined that the sediment of TC3 was composed of a background region with an intrusion consisting of a rectangular block located in range between 1100 $\mathrm{m}<\mathrm{R}<2900 \mathrm{~m}$. This result was confirmed by the workshop organizers when the input models were revealed for each of the test cases. Having identified the different regions, wavenumber estimates were made using the entire aperture of data within the 


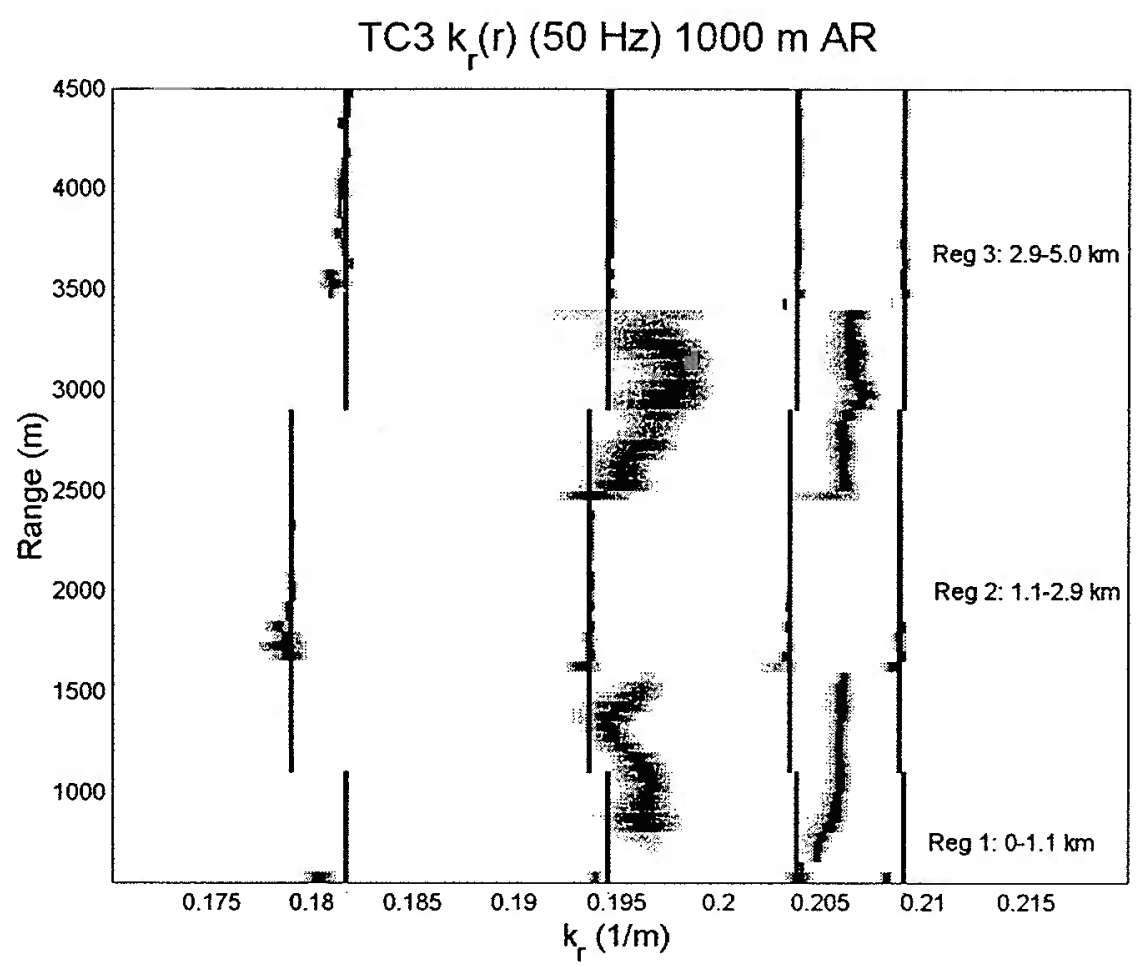

Figure 3-15: Wavenumber estimates for range-dependent sediment of Inversion Workshop TC3 with range-independent segments identified.

3 range-independent segments as shown in figure $3-16$. The individual wavenumbers estimated for the intrusion are all less than the corresponding values estimated for the background sediment of regions 1 and 3 . This indicates that the intrusion consists of a harder material relative to its surroundings and has a correspondingly higher sound speed. Using the perturbative inverse method previously described, this result was confirmed. Figure 3-17 shows the inverted sound speed profile (dashed) along with the starting background profile (circles) and true answer (solid) for each of the regions.

The inversion was only performed at a single frequency, and does not capture the detailed nature of the true profile, but matches well in an average sense. Resolution kernels [65] for each inversion confirm this and are shown in figure 3-18 for several depths indicated by $z_{0}$. In general, they indicate resolution is better at the shallower depths as indicated by the sharper peaks. Higher resolution could be obtained by performing wavenumber estimates and inversions at higher frequencies. This was 

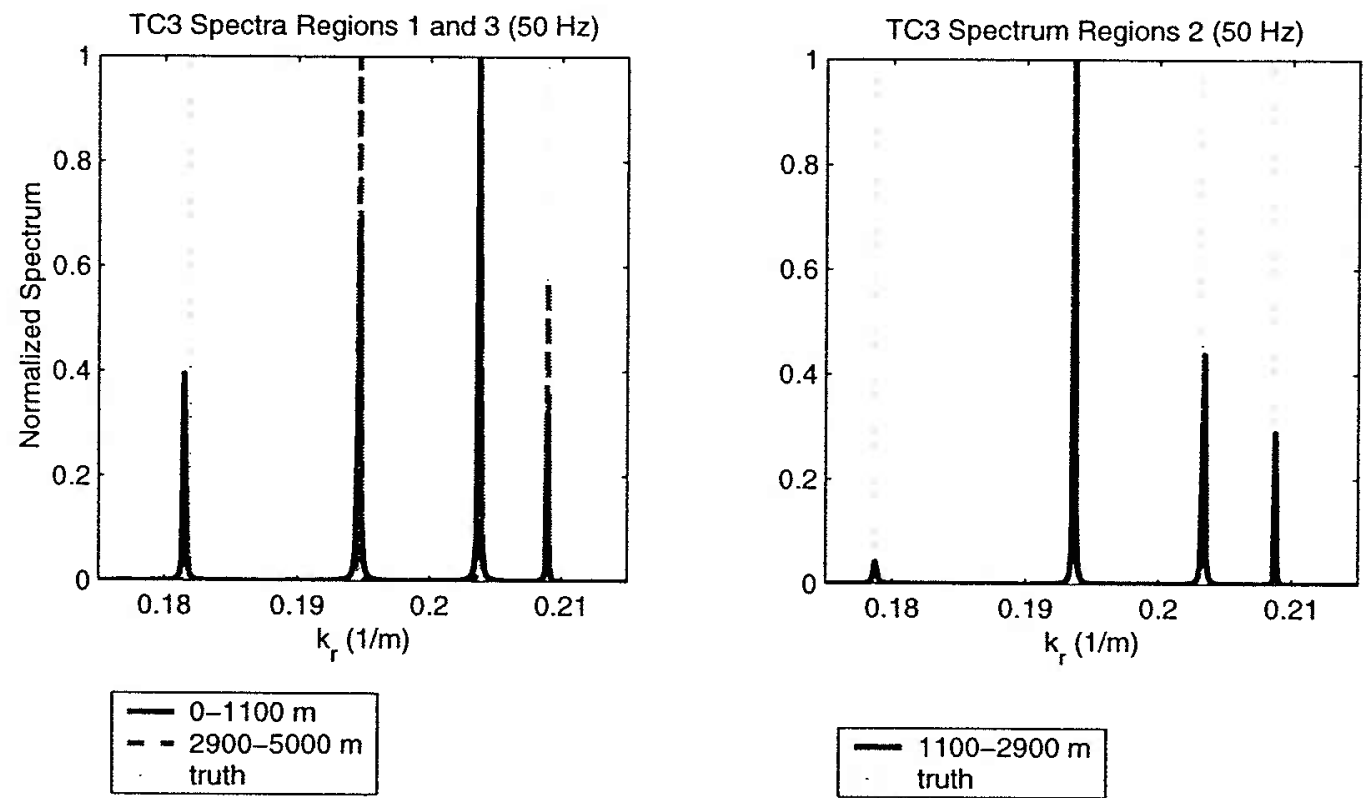

Figure 3-16: Spectral estimates for full apertures of range-independent regions identified for Inversion Workshop TC3.
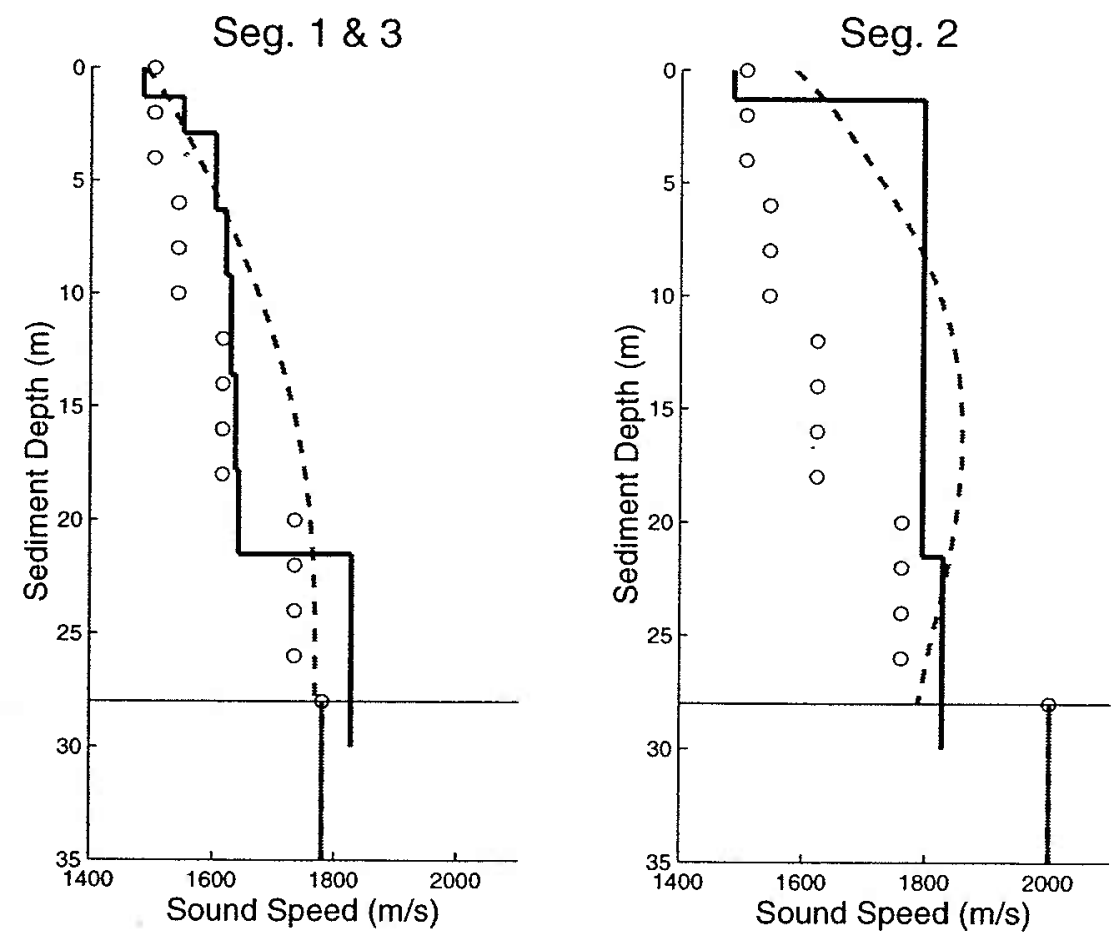

Figure 3-17: Compressional wave speed in the sediments for Inversion Workshop TC3. Open circles are background profile used for perturbative inversion, solid line is ground truth, and dashed line is inferred profile. 

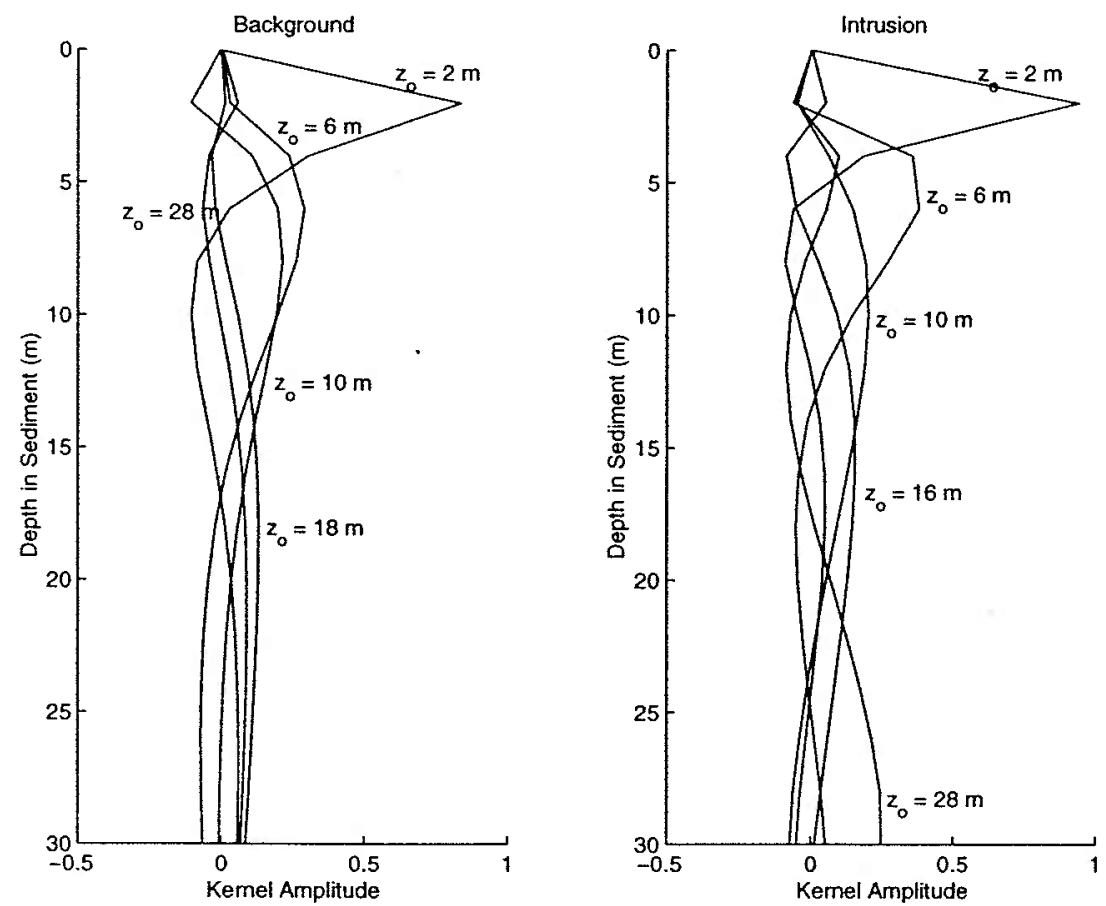

Figure 3-18: Resolution Kernels for inversion results of Inversion Workshop TC3. Background represents regions 1 and 3 and Intrusion represents region 2.

not done due to time constraints, and because the data were not provided on a dense enough range grid to estimate wavenumbers without aliasing. Comparison of complex pressure calculated for the inverted profile matched well the experimental data at 50 $\mathrm{Hz}$ and $100 \mathrm{~Hz}$ and shown in figures 3-19 and 3-20. A conclusion from this test case is that high-resolution estimators are necessary in order to resolve discrete events that occur in the sediment and impact on the complex pressure field. It is clear from the analysis in figures 3-1-3-3 that the intrusion in TC3 could not be resolved using classical techniques. 

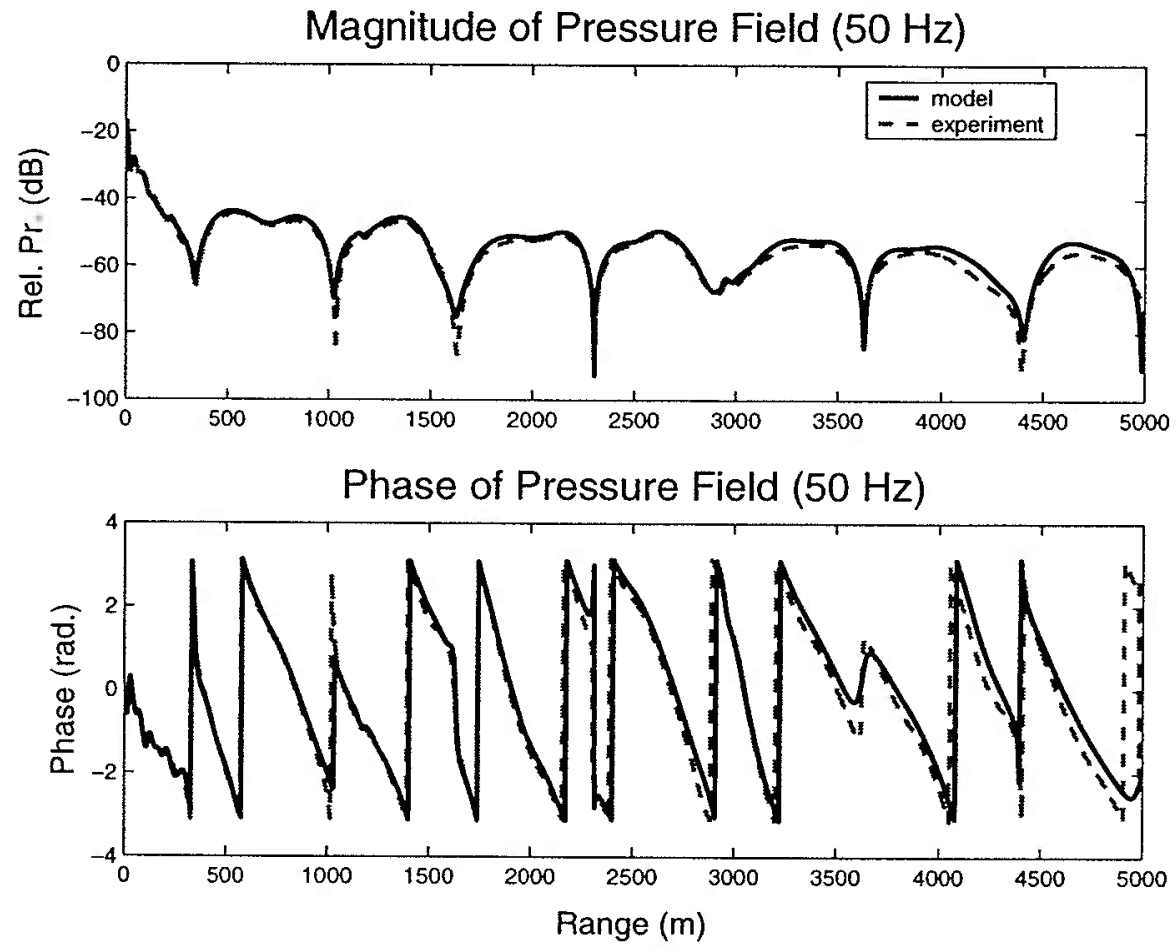

Figure 3-19: Complex pressure field modeled using inferred sediment sound speed profile compared to inversion workshop TC3 data at $50 \mathrm{~Hz}$. 

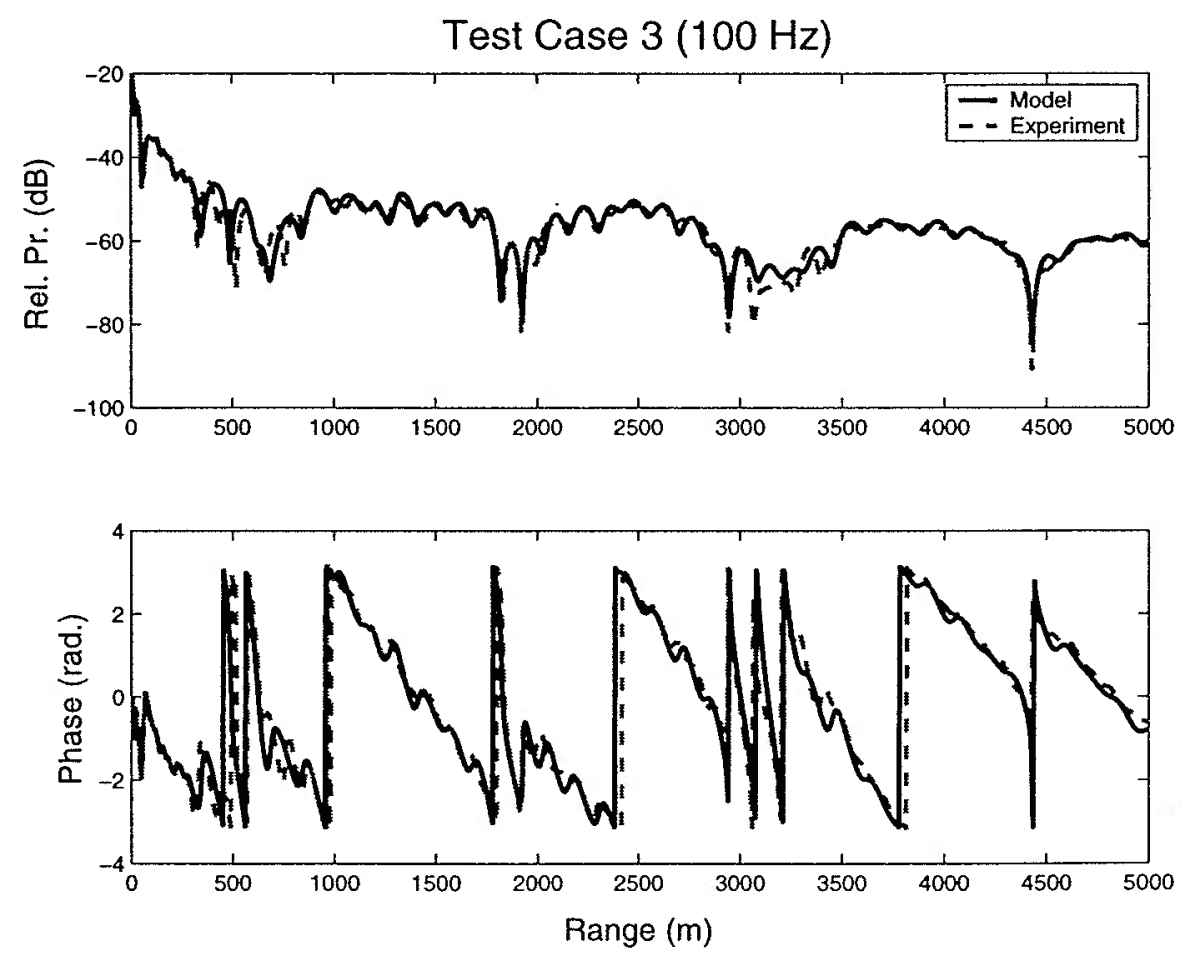

Figure 3-20: Complex pressure field modeled using inferred sediment sound speed profile compared to inversion workshop TC3 data at $100 \mathrm{~Hz}$. 


\subsection{Wavenumber estimation in random media - In- ternal Waves}

In addition to making wavenumber estimates for waveguides with range varying bathymetry and sediments, measurements in realistic ocean environments include the effects of sound speed variability in the watercolumn due to temperature fluctuations. Although, small sound speed perturbations may be indistinguishable from other causes of observation noise in the data - such as small ranging errors or rangedependence in the sediments or bathymetry due to sand ripples - where estimator characteristics have already been described, larger variations must be considered. This is particularly true in the case of internal waves (IW), where it is well known that energy transfer can occur amongst the propagating modes [61]. The temporal measure of sound speed variability passing due to internal waves is on the order of several minutes compared to a few seconds for a propagating acoustic field to pass through a given point [14]. By propagating over several kilometers through multiple internal wave events, the received field takes on features of a random process. In a recent paper, Siderius et. al. [74] look at the effect of sound speed fluctuations on geoacoustic inversion results for broadband acoustic data measured on vertical line arrays. In the wavenumber analysis discussed for this thesis and the experimental work, the effects of internal waves are assumed to be minimized due to the averaging effect that occurs when transforming the data over a long aperture in range [14]. Further, in the previous discussion, modal evolution was discussed in the context of adiabatic mode theory where no energy is transferred between modes and the number of propagating modes remains constant. However, in the presence of internal waves the adiabatic mode assumption is violated and the effects on local wavenumber estimates should be understood. In particular it is of interest to determine if internal wave fields cause a shift in the estimated wavenumbers, if they cause certain modes not to be resolved, or if additional modes are excited and can be detected. 
To understand the effects of internal waves on the forward propagation problem, a recent session at a meeting of the Acoustical Society of America (ASA) [81] focused on benchmarking acoustic propagation models for range-dependent media including internal waves. Although focused on the forward problem, these studies provided valuable data for beginning to understand the wavenumber estimation problem.

In this section, synthetic data from the benchmark session is used for estimating wavenumber content for an environment both with and without internal waves. A description of this model and other range-dependent cases can be found at [75]. The waveguide environment was a Pekeris model with a flat bottom at $200 \mathrm{~m}$ depth. Density in the watercolumn was $1 \mathrm{~g} / \mathrm{cm}^{3}$ with no attenuation. The bottom was modeled as a fluid with sound speed of $1700 \mathrm{~m} / \mathrm{s}$, density $1.5 \mathrm{~g} / \mathrm{cm}^{3}$, and attenuation $0.1 \mathrm{~dB} / \lambda$. The background sound speed profile was generally downward refracting with a slight duct occurring above $26 \mathrm{~m}$ depth and was given by [76]

$$
\begin{aligned}
& c(z)=\quad 1515+0.016 z, \quad z<26 m \\
& c(z)=c_{o}\left[1+a\left(e^{-b}+b-1\right)\right], \quad z>=26 m,
\end{aligned}
$$

where $c_{o}=1490 \mathrm{~m} / \mathrm{s}, \mathrm{a}=0.25$, and $\mathrm{b}=(\mathrm{z}-200) / 500[76]$.

To the background sound speed was added a perturbation as a function of depth and range to represent the internal wave field. The sound speed perturbation, $d c$, was given by,

$$
d c(z, r)=4(z / B) e^{-z / B} \sum_{i=1}^{5} \cos \left(K_{i} r\right),
$$

where $K_{i}=2 \pi[2000-300(i-1)]^{-1} \mathrm{~m}^{-1}$ and $\mathrm{B}=25 \mathrm{~m}$. Using this model, the maximum sound speed perturbation is about $7.5 \mathrm{~m} / \mathrm{s}$. The range dependent sound speed profile is shown in figure 3-21. Using the above models, PECan [11], was used to generate the acoustic field for both the background and perturbed sound speed environments from $0-20 \mathrm{~km}$. The data are shown in figure 3-22 for the full aperture and for $5 \mathrm{~km}$ sub-apertures. The difference in structure of the modal interference 

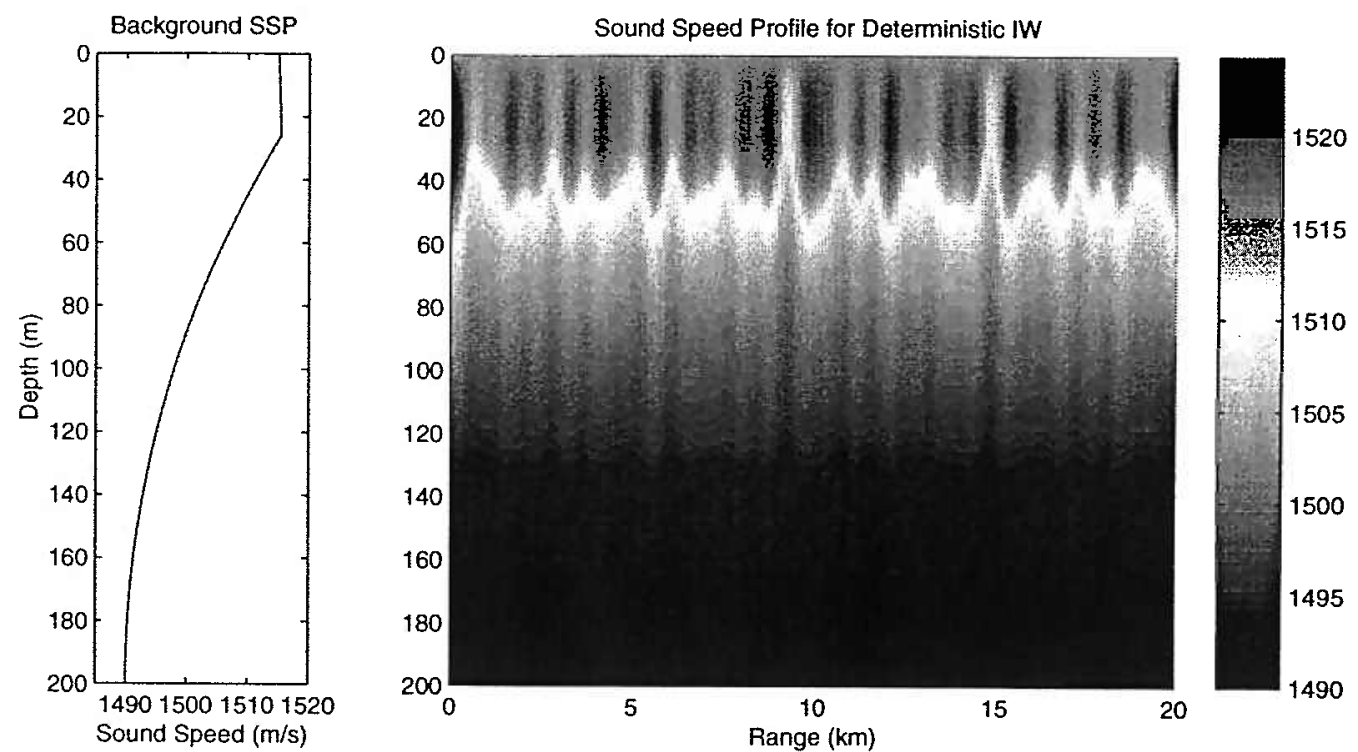

Figure 3-21: Range-dependent sound velocity field for IW wavenumber estimates [76]. Background profile is on left.

patterns observed in the transmission loss plots is most evident at long ranges. These data were generated by Gordon Ebbesen [21] at DREA Canada on a 5 meter range grid. $3000 \mathrm{~m}$ sliding window AR wavenumber estimates were made for both cases with a step of $500 \mathrm{~m}$ and a model oder of 200 . Additionally, the exact mode values for the unperturbed model environment were determined using KRAKEN. The resulting wavenumber results with the KRAKEN results overlayed are shown in figures 3-23 and 3-24.

The number of modes determined by KRAKEN is greater then the number estimated using the sliding window method indicating that not all the modes are excited. Wavenumber estimates that agree with the KRAKEN results are shown by a dashed line. The plots show that for the unperturbed case, 6 modes are estimated that can be correlated with the KRAKEN result. For the IW case, 8 modes are identified that correlate with the KRAKEN results. The plots show the wavenumbers to be very stable with range, indicating range-independent boundary conditions. This is particularly evident for the the higher order modes. There is evidence of some instability of the estimates for the IW case seen as undulations about the predicted 
a) TL vs Range $(100 \mathrm{~Hz})$

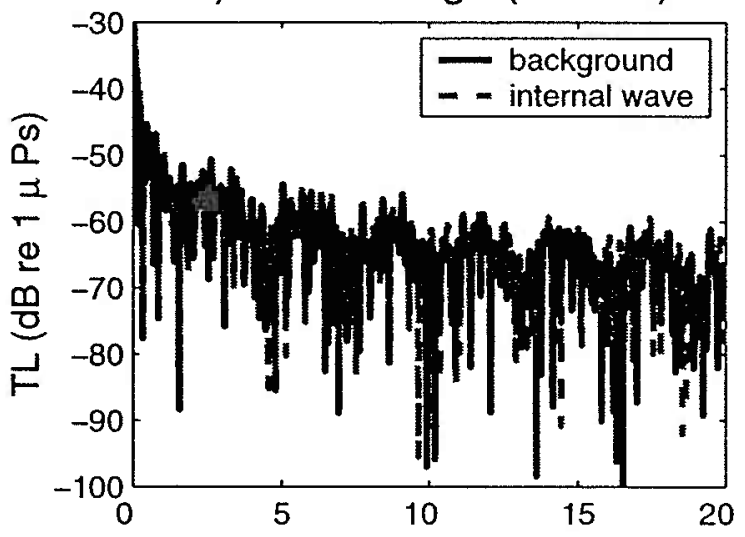

c) $10-15 \mathrm{~km}(100 \mathrm{~Hz})$

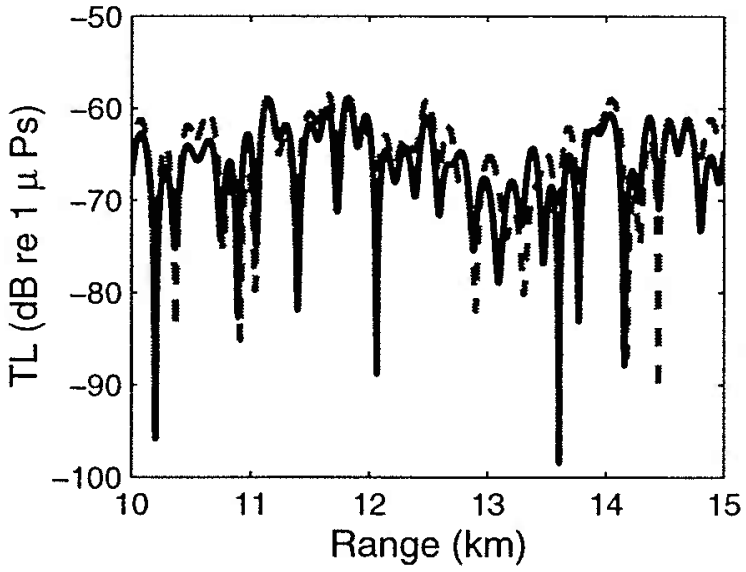

b) $0-5 \mathrm{~km}(100 \mathrm{~Hz})$

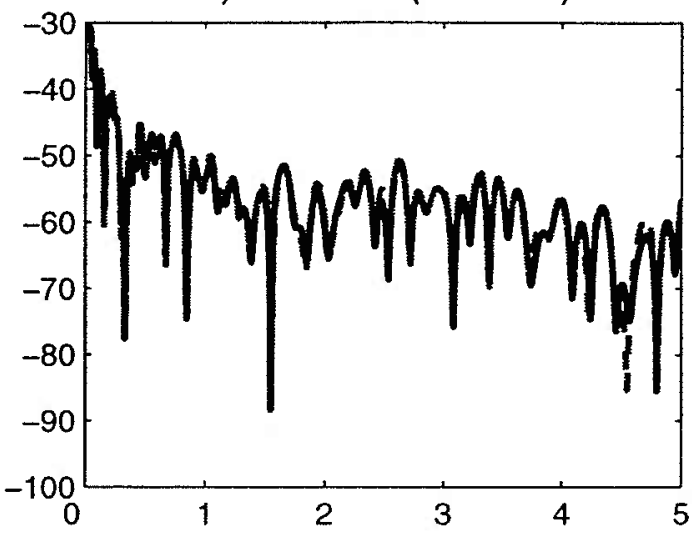

d) $15-20 \mathrm{~km}(100 \mathrm{~Hz})$

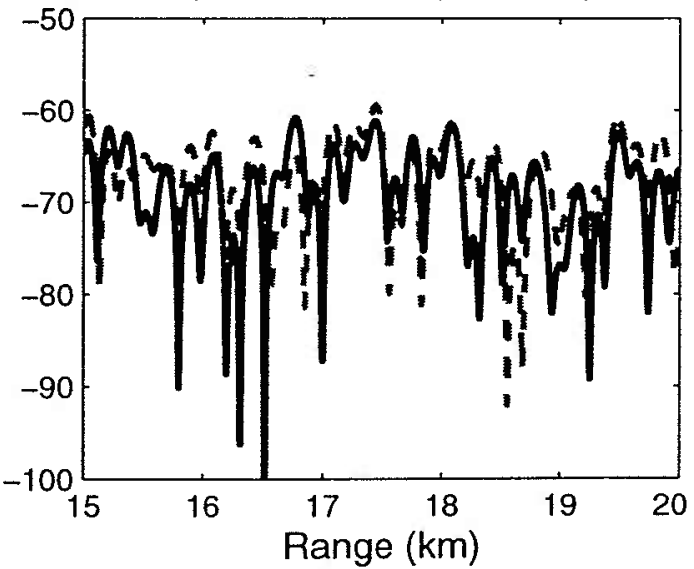

Figure 3-22: $100 \mathrm{~Hz}$ TL plots for internal wave study. $5 \mathrm{~km}$ sub-plots show departure of internal wave measurements from unperturbed case. 


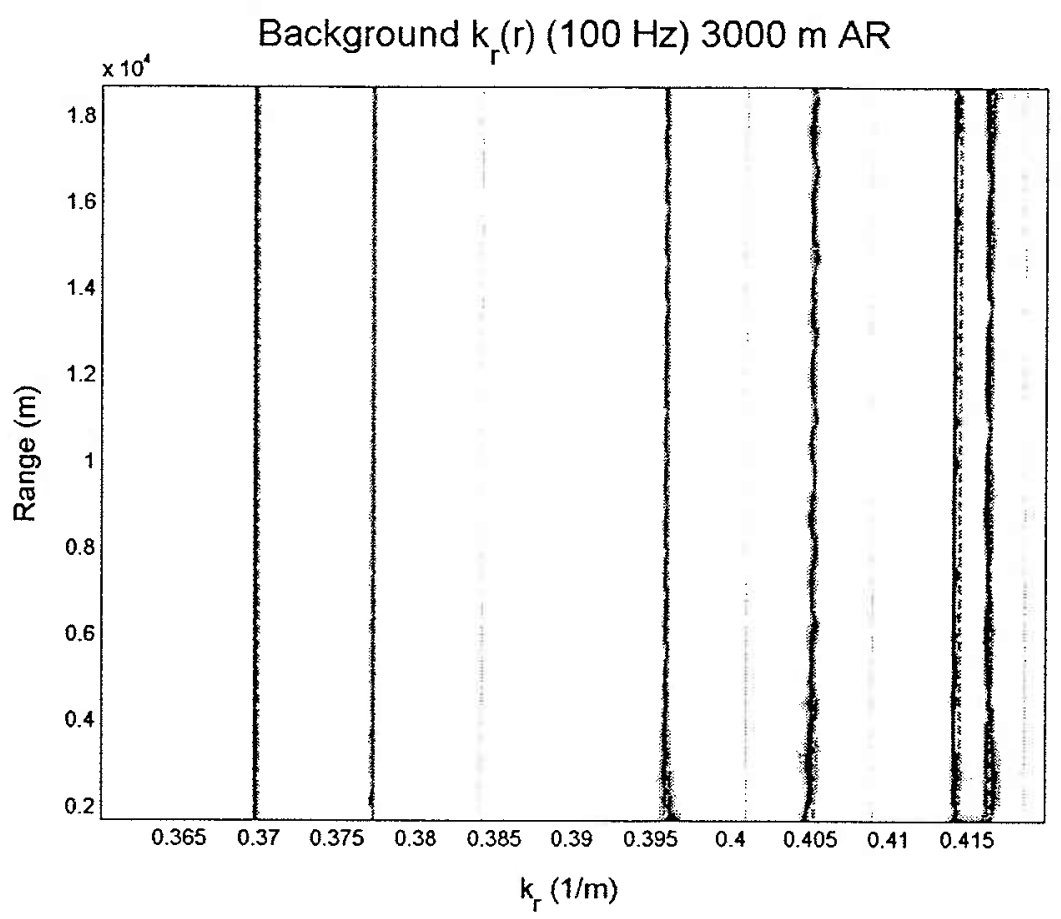

Figure 3-23: Sliding window wavenumber estimates with KRAKEN results (dashed) plotted for unperturbed case.

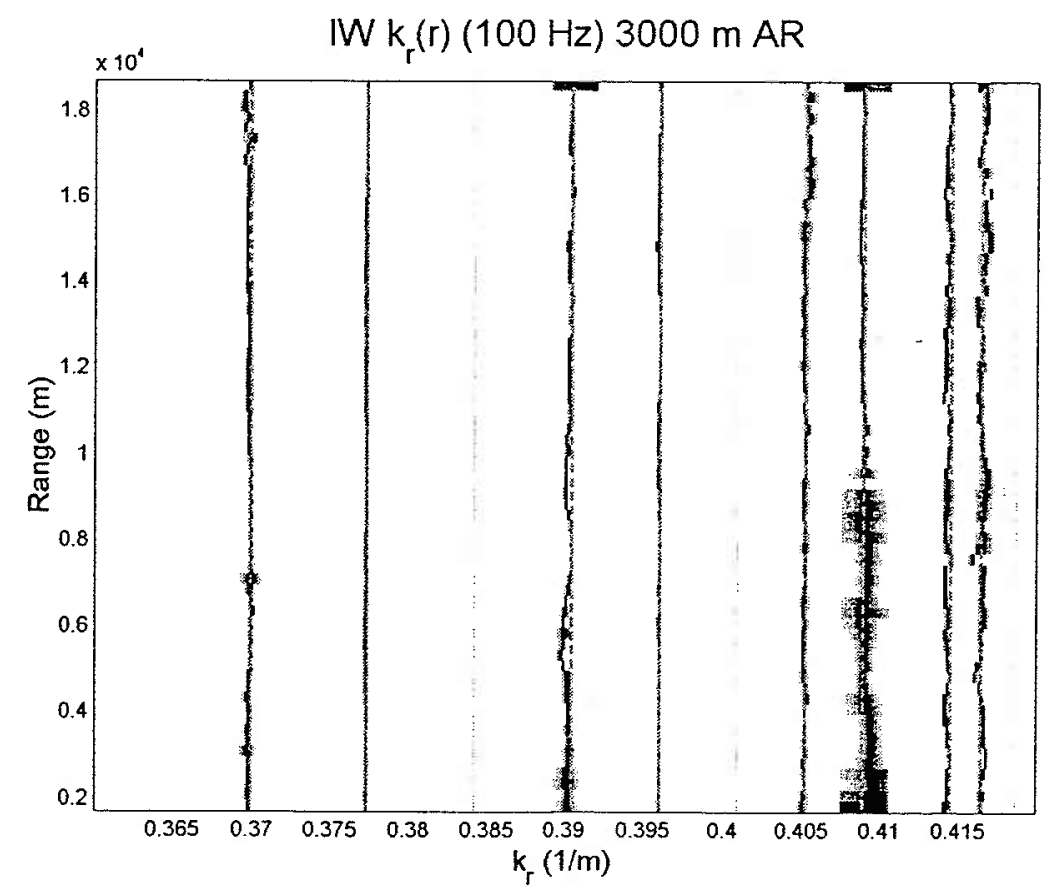

Figure 3-24: Sliding window wavenumber estimates with KRAKEN results (dashed) plotted for IW case. 
value. However, this result might be expected as energy is being exchanged between the modes with range and appears as a scintillation in the wavenumber estimates with range. However, there is no apparent shift observed for the modes observed in the unperturbed case and the IW case. There are, however, additional modes that are energized due to the mode coupling which are consistent with those predicted by KRAKEN. A conclusion from this study is that for the environment provided, the internal waves couple energy into modes that might not otherwise be excited for a given source/receiver geometry and give additional information that could be used as input data for inversion. This is a good result for the relatively weak internal wave field provided. However, future studies could include an examination of the types of limits on IW perturbations that cause these observations to break down. A second caveat to these observations relies on the ability of an estimator to resolve individual modes. The exact nature of the coupling and the wavelength of the internal wave structure will impact the ability to resolve modes. In this particular case, it is clear that the first two modes predicted by KRAKEN were not present in the data. However, the first two modes were very close together in wavenumber space and would require a long aperture to resolve them. These issues are less troublesome at lower frequencies and shallower depths where the wavenumbers are less densely spaced. In these cases, shorter apertures could be used to observe the same effect as the $3 \mathrm{~km}$ apertures used in this study. 


\section{Chapter 4}

\section{Modal Mapping Experiment (MOMAX)}

\subsection{Introduction}

The Modal Mapping Experiments were designed as an efficient means for measuring the full spatial variability of a point source acoustic field in a complex shallow water environment [30]. Previous experimental efforts have, in general, employed synthetic aperture horizontal arrays generated along radials to measure pressure as a function of range at a constant bearing. Typically, this was achieved by towing a hydrophone, suspended at a fixed depth, away from a moored source, or alternatively; towing a source away from a fixed receiver. For ranges greater than a few wavelengths, the horizontal wavenumber spectrum of the field could then be extracted by employing the asymptotic form of the Hankel transform. These efforts were effective in demonstrating the range-dependent nature of the spectrum due to range dependent waveguide properties. However, it is desirable to examine the effect of waveguide properties on the spectrum along radials at all angles around the source as given from a complete spatial measure of the propagating field. The MOMAX experiments were designed to make measurements of the propagating acoustic field for a point source on a spatial 

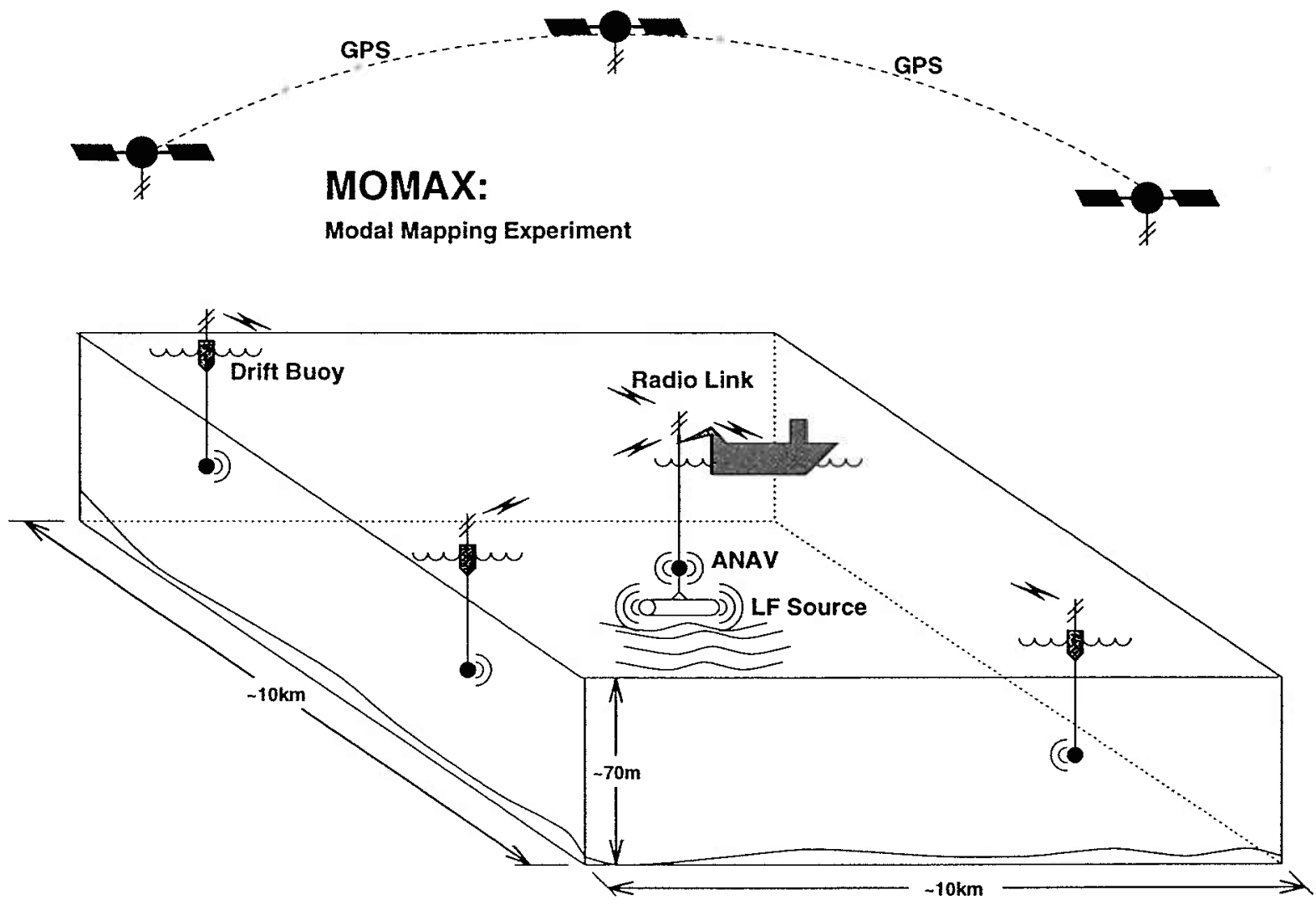

Figure 4-1: Sketch representation of MOMAX experimental layout

grid given in absolute geospatial coordinates of latitude and longitude. By design, these measurements are related to the horizontal wavenumber spectrum of the field by application of the appropriate two-dimensional transform relation as discussed in chapter 2. An overview of the experimental configuration is given in the next section followed by descriptions of specific experiments.

\subsection{Experiment Overview}

In the MOMAX experiments, data are acquired on an array formed by individual hydrophones suspended from several freely drifting buoys equipped with precision GPS navigation. Figure 4-1 illustrates the operational scenario and assets. As many as four buoys might be drifting at a given time and synthetic apertures can be formed 
using data acquired on a single buoy or several buoys.

For the usual experimental run, the acoustic field was generated by a single projector operating in a cw mode comprised of several discrete tones between 20 and 475 $\mathrm{Hz}$. At any given time, no more then four frequencies were transmitted by a projector. During one experiment, two projectors were used simultaneously transmitting four frequencies each. Several different acoustic projectors were used in the MOMAX experiments, including an NRL J-15-3 source (50-475 Hz), a Webb Research organ pipe type source $(200,300 \mathrm{~Hz})$, and an IHI source $(20-125 \mathrm{~Hz})$. Each source was set to operate at a nominal level of $173 \mathrm{~dB}$ re $1 \mu \mathrm{Pa}$ at the set frequencies. Experiments were run with the source(s) either moored on the seafloor, or suspended from the ship at fixed depth - nominally 30 meters. With the source suspended from the ship, several different operational configurations were used including mooring the ship to achieve a stationary source position, allowing the ship to drift freely, or towing the source with the ship. These configurations play an important role in the interpretation of the data as will be shown in the next chapter.

The measurement buoys used in MOMAX, schematically depicted in Figure 4-2, were developed at WHOI specifically for these experiments. Each buoy contained a calibrated hydrophone suspended at a nominal depth of 30 meters by a system designed to isolate the effects of wave motion on the vertical motion of the phone. Measurements of hydrophone depth using pressure sensors showed actual hydrophone depths to be greater than 30 meters, but generally stable, keeping depth to within a meter. A typical depth record for a single experiment is plotted in figure 4-3. The data record includes data taken starting before deployment and continuing through recovery of the buoys. A depth reading of zero is indicated when the buoy is on deck. Throughout the course of the experiment, it was observed that during periods of adverse weather at the surface, hydrophone depth variation increased as the limits of the damping system were exceeded. It should be noted that between the second MOMAX experiment and the SWAT experiment, the hydrophone suspension and 


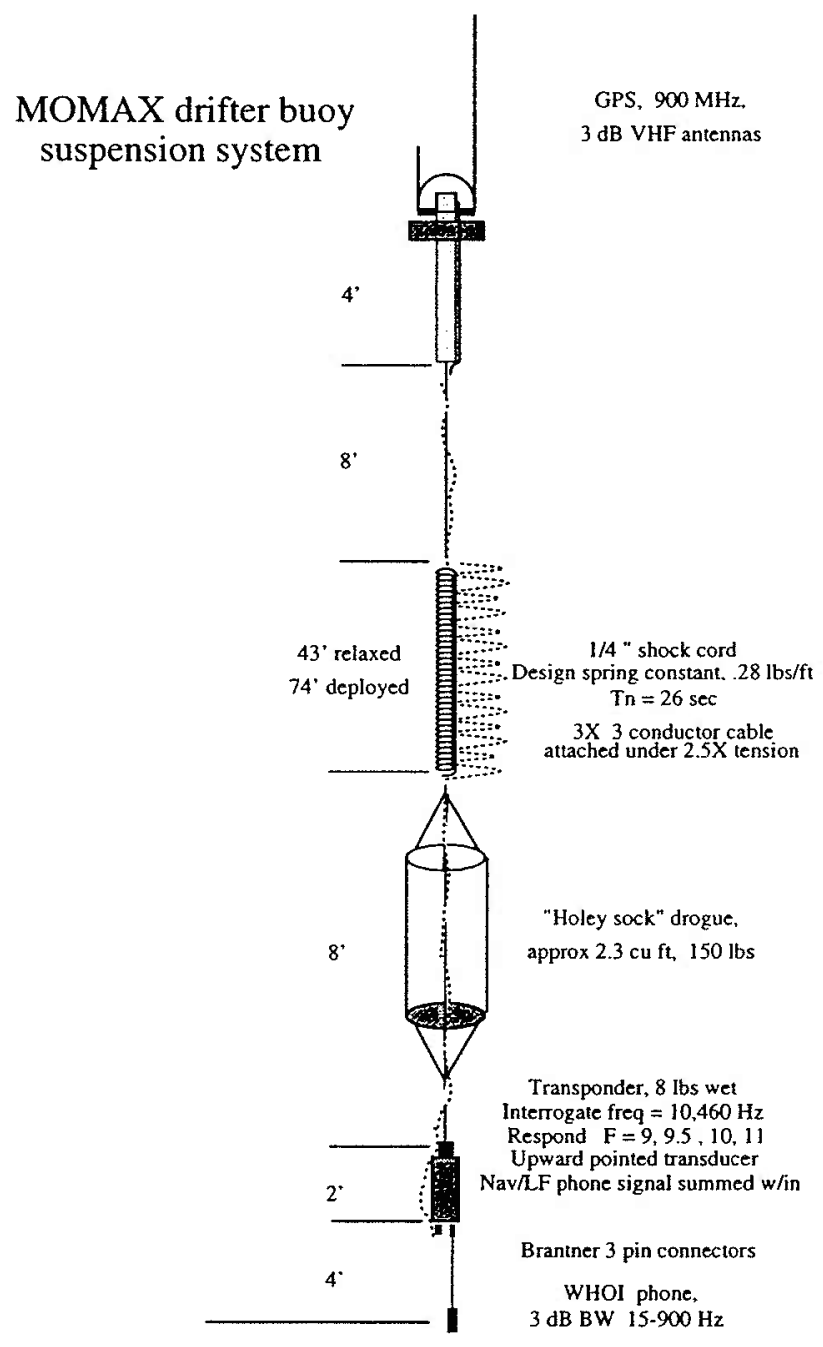

Figure 4-2: MOMAX drifter buoy components. 


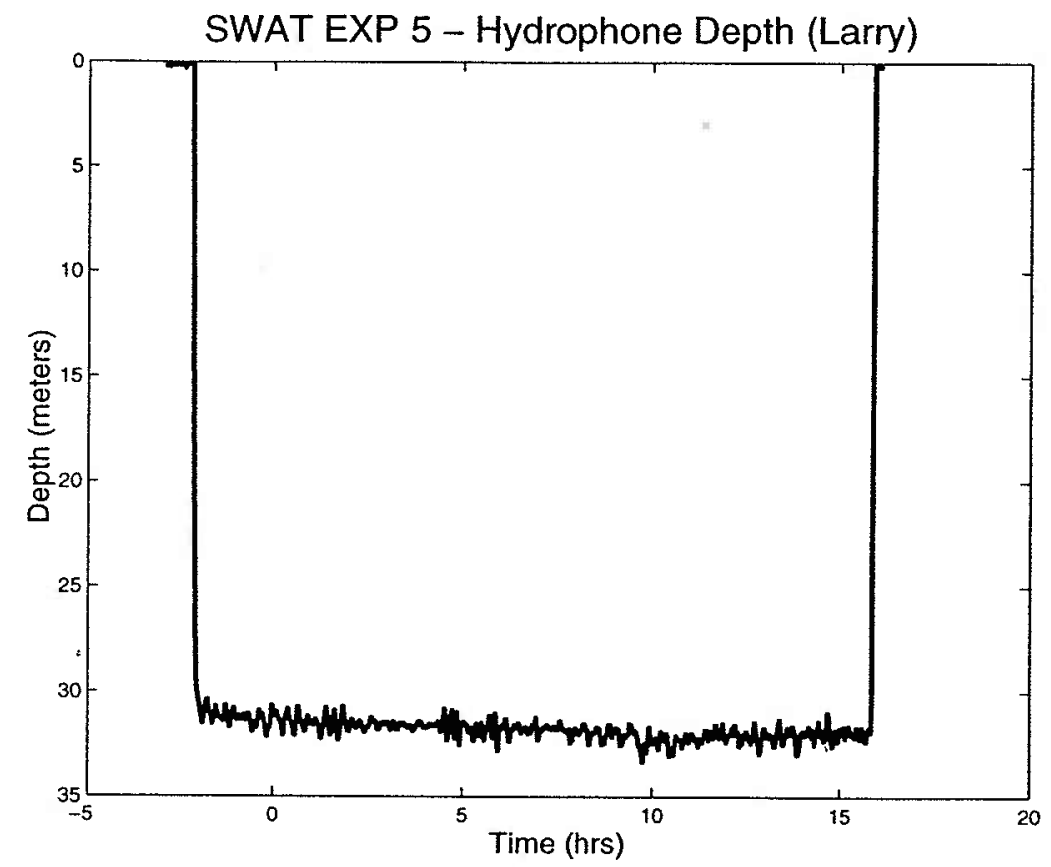

Figure 4-3: Hydrophone depth record for typical MOMAX experimental run.

damping system was modified. Comparing depth records from both experiments, the system used on the original buoys was superior to that of the SWAT experiment. This observation is important because depth data was not available for the MOMAX 97 experiment that used the original hydrophone suspension system.

Acoustic data measured at the phones were broadcast back to the ship in realtime over a VHF radio link. The analog signals were sampled at $3255.2083333 \mathrm{~Hz}$ and stored to computer hard disk. The buoys were also equipped with GPS receivers and $900 \mathrm{MHz}$ modems for transmitting geographic coordinates back to the ship in real-time. Using a mapping program developed as part of this work, a real-time display of all buoy and ship positions was used to monitor buoy locations. Additional GPS receivers were used to measure the position of the suspended acoustic sources. GPS data were recorded and subsequently processed to achieve absolute positioning accuracy to within 1 meter. Further, by establishing a local differential GPS system between the source ship and each buoy, relative positions between the source and each receiver were measured to sub-meter accuracy [19] [20].

Application of Hankel transform based methods to the measured data requires 
accurate measurements of the acoustic phase; consequently a precise time base was required for both the source and receiver data. The source signal was generated by a function generator with an external clock controlled by a TRAK GPS clock accurate to within 200 nanoseconds of true GPS time. The same clock was used to drive the data acquisition system. Synchronization of the time base for the source and receiver systems allowed very precise phase measurements to be made of the acoustic signal.

\subsubsection{Data Reduction}

The acoustic signal was generated and transmitted as a sum of sinusoids for the frequencies of interest. However, the Hankel transform method described is for a single frequency. To look at a single frequency, the data received on the hydrophone were quadrature demodulated at each of the transmitted frequencies [5] by taking the FFT of approximately 1.25 seconds of data with time determined by the center of the FFT window. Complex pressure data at each frequency were taken as the real and imaginary parts of the resulting transform at the appropriate frequency bins. Care was taken to ensure that the resulting spatial sampling was on the order of a point every meter. The total signal is treated by sliding a window of a given number of points and performing the FFT at each step. A half-window overlap was used for this procedure. Phase accumulation was accounted for by keeping track of time accumulated before application of the transform at each step and-wrapping accordingly.

Positioning data were recorded independently on a separate system. The acoustic and navigation data were merged by finding the union of times where both acoustic and position data were recorded. Positioning data were acquired every second, a slightly faster rate then the demodulated acoustic data. Consequently, the acoustic data was interpolated onto a time grid common to the GPS data. Linear interpolation was used by considering the real and imaginary parts of the complex signal separately. The result of this step was to provide data on an xy-grid relative to the source 
positioned at the origin. Similarly, the data could be interpolated onto an absolute position grid by using the GPS data given in coordinates of latitude and longitude.

\subsection{MOMAX 97 and SWAT 01}

Since 1997, three different MOMAX type experiments have been performed. Two of the experiments took place off the coast of New Jersey and will be discussed in this thesis. The third experiment took place in the Gulf of Mexico, about $145 \mathrm{~nm}$ west/northwest of Key West, Florida.

\subsubsection{Environment}

The first modal mapping experiment took place in about 70 meters of water off the NJ coast in March 1997. In October 2001, another experiment was conducted in the same area under the Shallow Water Acoustic Technology (SWAT) Memorandum of Understanding (MOU) between the US and Japan. The overall SWAT experiment was a joint effort between institutions from both the US and Japan. In addition to the MOMAX group from WHOI, other US participants were the Naval Research Laboratory (NRL), and the University of Miami (UM). The Japanese participants were under the direction of the Technical Research and Development Institute (TRDI).

The general operational area for both experiments was within the STRATAFORM swath mapping survey area shown in figure 4-4. STRATAFORM is a program of the Office of Naval Research (ONR) to collect high-resolution geophysical data in shallow-water regions for determining continental shelf morphology and sediment properties [53]. Presently there is a concerted ONR effort [56] by both geologists and underwater acousticians to characterize this region in terms of both its geologic and acoustic properties. The MOMAX effort is concentrated on examining the relationship between local sub-bottom sediment properties and low-frequency acoustic propagation. 


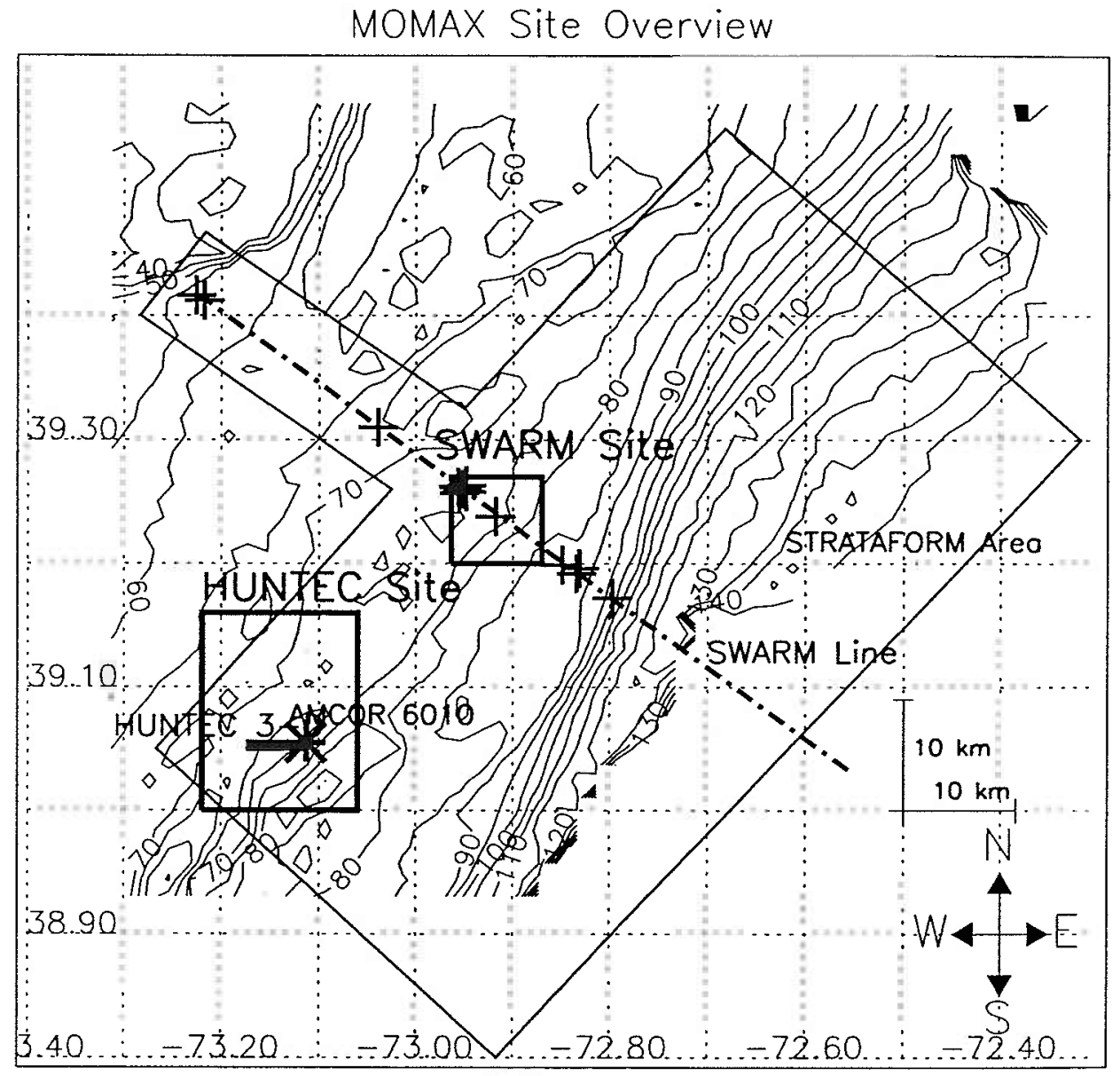

Figure 4-4: MOMAX Experiment Site 
Specifically, MOMAX 97 was concentrated in two regions within the STRATAFORM area centered at $73.17^{\circ} \mathrm{W}$ Longitude, $39.06^{\circ} \mathrm{N}$ Latitude and $72.91^{\circ} \mathrm{W}$ Longitude, $39.24^{\circ} \mathrm{N}$ Latitude corresponding to the HUNTEC site and SWARM site, respectively. The HUNTEC site was where an extensive high-resolution 3-D seismic survey was conducted with the discovery of a complex internal sediment structure underlying a more benign seafloor surface [16]. SWARM refers to the "Shallow Water Acoustic in Random Medium" experiment that took place in 1995 [3]. The SWAT experiment revisited the HUNTEC and SWARM sites with focal points near $73.15^{\circ} \mathrm{W}, 39.00^{\circ} \mathrm{N}$ and $72.85^{\circ} \mathrm{W}, 39.30^{\circ} \mathrm{N}$, respectively. Each of these locations is within a region referred to as the "outer shelf sediment wedge" [16]. This region extends along the edge of the New Jersey shelf for $150 \mathrm{~km}$ southwest of Hudson Canyon. The entire region can be characterized by a layer of soft sediments of varying degrees of thickness and stratification overlying a prominent sub-surface seismic reflector, designated " $R$ ". In the region of the MOMAX experiments the sediment layer is thin and is thought to be a homogeneous mixture of silty and sandy clay [16]. Additionally, 3-D seismic reflection surveys have identified regions within the wedge where the sediment layer is patchy. In some regions, the reflector $\mathrm{R}$ breaks the surface and sub- $\mathrm{R}$ sediments are exposed. In these regions a complex system of sub-marine channels has been identified that are believed to have been caused by an erosional episode which occurred during the last (Wisconsinan) period of low sea level. Bathymetry for the area is relatively benign with depth gradually increasing along lines perpendicular to the shelf break.

\subsection{Summary of Measurements}

\subsubsection{MOMAX 97}

During the course of MOMAX 97, a total of 8 separate experiments were conducted. Experiments 1-7 were done in the vicinity of HUNTEC, and experiment 8 was conducted at the SWARM site. Table 4.1 provides a summary of the different experiments 


\begin{tabular}{|c|c|c|c|c|c|}
\hline Exp. No. & Site & Source & Moe & Curley & Shemp \\
\hline \hline 1 & HUNTEC & J-15 & no & no & YES \\
2 & HUNTEC & J-15 & no & no & YES \\
3 & HUNTEC & J-15 & YES & YES & no \\
4 & HUNTEC & J-15 & YES & YES & YES \\
5 & HUNTEC & WEBB & YES & YES & YES \\
6 & HUNTEC & J-15 & YES & YES & YES \\
$7($ a \& b) & HUNTEC & WEBB & YES & YES & YES \\
8 & SWARM & J-15 & YES & YES & YES \\
\hline
\end{tabular}

Table 4.1: Overview of source and buoy deployments for MOMAX 97.

including site location along with the type of source and the buoys deployed. Source frequencies for the $\mathrm{J}-15$ were set at $50,75,125$, and $175 \mathrm{~Hz}$ for all experiments, and the Webb source operated at 200 and $300 \mathrm{~Hz}$.

Throughout the experiment, environmental data were collected in the form of CTD data for determining the sound velocity in the water column, as well as current data. Each buoy and the source were also equipped with temperature sensors that recorded temperature continuously throughout the experiments. As shown in Figure 4-5, sound velocity in the water column was generally isovelocity to a depth of between $30-35$ meters, upward refracting between 35-60 meters, and isovelocity for depths greater then about 60 meters. In addition to the water column measurements, chirp sonar data were collected along the drift tracks of the individual buoys and the source for many of the experiments. The chirp was operated for a frequency range of $3-6 \mathrm{kHz}$ and was to provide detailed bathymetry data over the course of each experiment. Additionally, the chirp data could be used to indicate qualitatively the stratigraphy in the region of the individual experiments. Unfortunately, for this experiment, data recorded on DAT tapes for archiving were not usable for further analysis so no quantitative analysis of the CHIRP data was possible. However, as part of the SWAT experiment, a separate CHIRP survey was conducted in the STRATAFORM area in the vicinity of HUNTEC and SWARM. As this data becomes available, regions of 

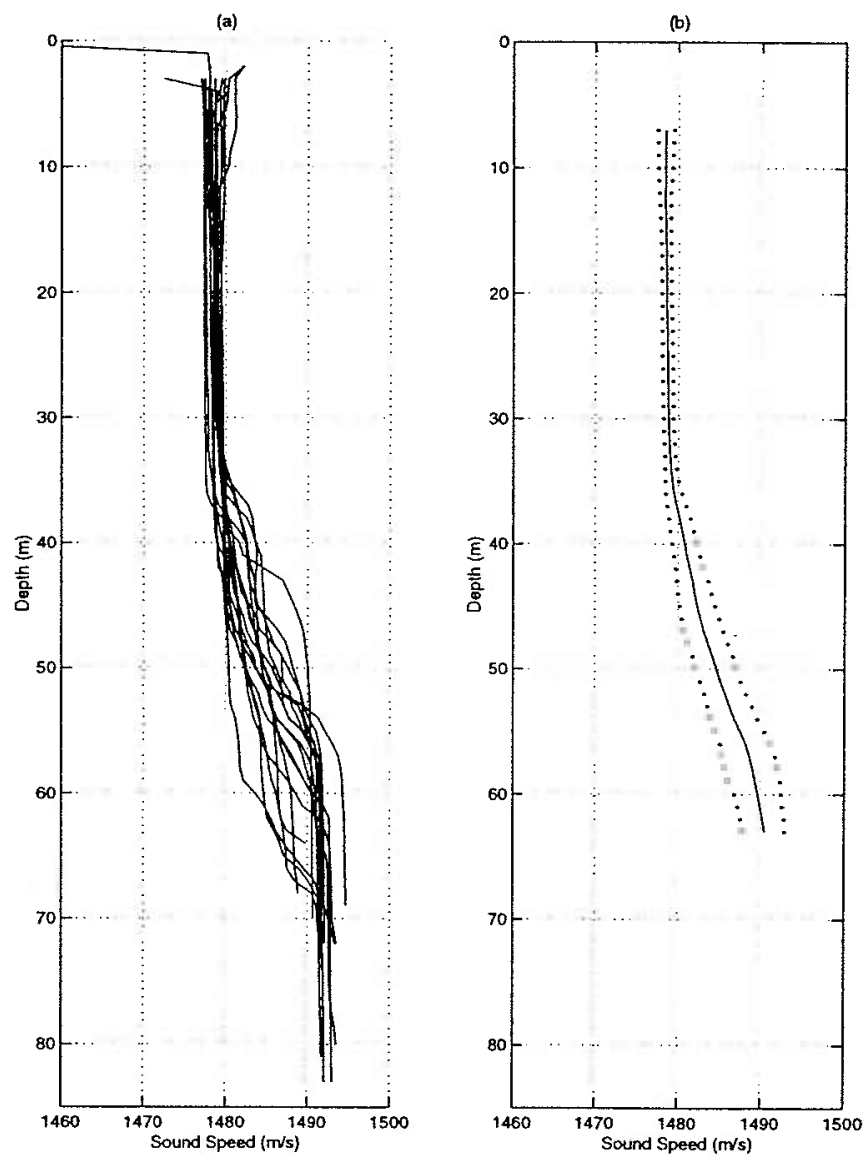

Figure 4-5: (a) SSP for all experiments (b) Mean and $+/-1$ std 


\begin{tabular}{|c|c|c|c|c|c|c|}
\hline Exp. No. & Site & Source & Source Freqs. & Moe & Larry & Shemp \\
\hline \hline 1 & SWARM & IHI & $20,50,75,125$, & YES & no & YES \\
& & J-15 & $175,225,425,475$ & & & \\
2 & SWARM & J-15 & $50,125,475$ & no & no & YES \\
3 & HUNTEC & J-15 & $50,75,125,175$ & YES & YES & YES \\
4 & HUNTEC & J-15 & $50,75,125$ & YES & YES & YES \\
5 & HUNTEC & J-15 & $50,75,125$ & YES & YES & YES \\
\hline
\end{tabular}

Table 4.2: Overview of source and buoy deployments for SWAT.

overlap with the MOMAX 97 experimental data will be sought out to supplement the environmental characterization.

Low-frequency acoustic data was taken at $20,50,75$, and $125 \mathrm{~Hz}$ with a source level of $174 \mathrm{~dB}$ re $1 \mu \mathrm{Pa}$ using the J-15 source suspended from the ship. It was intended that the ship be moored or at anchor to provide for stationary source measurements. However, due to weather conditions the ship could not be anchored and the experiments were conducted with the ship either drifting freely or towing the source at a constant speed. During experiments 5 and 7 the Webb source was moored and suspended about 1 meter off the seafloor and operated at 200 and $300 \mathrm{~Hz}$. Unfortunately, the internal clock on the Web source became unstable during the experiments and the data were unusable.

\subsubsection{SWAT}

The SWAT experiment was comprised of 5 different experiment runs, two near the SWARM site and 3 near HUNTEC. The source and buoy deployment overview is given in table 4.2. Frequencies are listed separately as the number and value of transmitting frequencies changed for each run. As originally planned, each of the SWAT experiments was to use two sources transmitted 4 frequencies simultaneously. Of particular interest, was the $20 \mathrm{~Hz}$ signal provided by the IHI source. Unfortunately, due to technical problems, the IHI source was only operable during the first run. As 


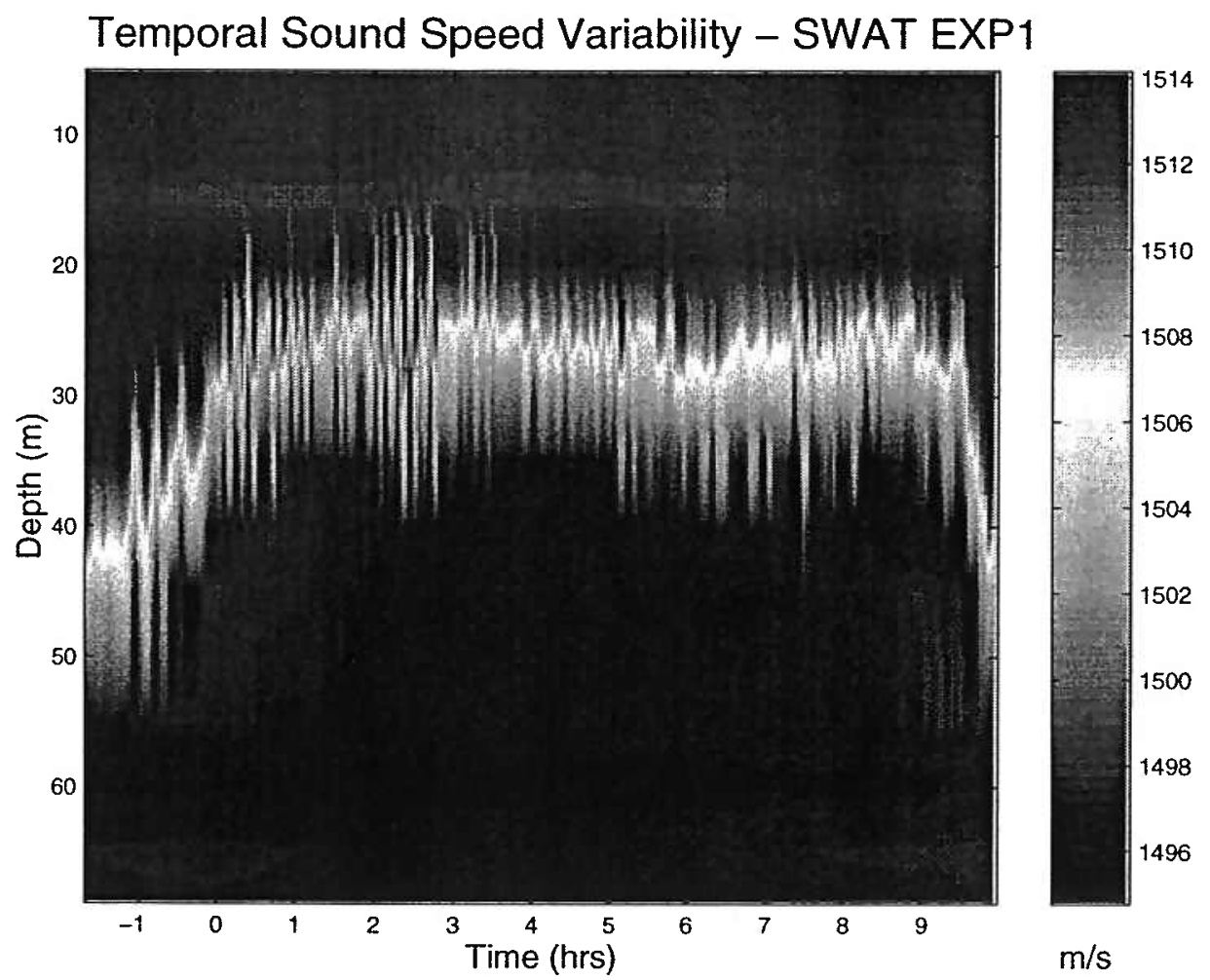

Figure 4-6: Sound speed variability during SWAT experiment 1 determined from temperature variability recorded on NRL T-string.

in MOMAX 97, the acoustic experiments were supported by a variety of environmental measurements including CTDs, XCTDs, and XBTs, along with temperature and depth sensors on both sources and receivers. Additional assets included stationary temperature string (T-string) moorings, deployed by NRL, that spanned the water column to provide a continuous record of the temperature variability during the experiment. Converting the temperature variations recorded on the T-string to sound speed using Wilson's equation [83], assuming a constant salinity value, an example of the sound speed variability measured during SWAT experiment 1 is shown in figure 4-6. Throughout the course of the experiment, the temperature profile showed the water column to be warm near the surface and cooler near the bottom indicating a downward refracting sound speed profile. As shown in the picture, the location of the boundary between the warm and cool mixed layers was time dependent with a vari- 
ation of 10 to 15 meters in depth over the course of the experiment. This variability would be of concern if performing wavenumber transforms over the entire data set. However, it will be shown for the data sets considered that the sound speed variability can be considered locally stationary.

High-resolution chirp sonar data and sediment cores were also performed in the area. These data are being analyzed separately and results are anticipated for future comparison with inversion results obtained as part of this thesis work. In the following chapter, data from the MOMAX 97 and SWAT experiments will be discussed and analyzed. 


\section{Chapter 5}

\section{Data Analysis}

\subsection{Introduction}

In this chapter some of the analysis techniques discussed in the previous chapters are applied to the MOMAX experimental data.

\subsection{Spatial measurements of complex-pressure fields}

The MOMAX experiments were novel in that they combined measurements of acoustic field data with navigation data provided by GPS. Previous synthetic aperture experiments typically used either acoustic or radar ranging systems to measure source/receiver separation distances [48]. The nominal accuracy provided by these systems was about one meter, where radar ranging was typically better at longer separation distances and acoustic methods used for near ranges. Using GPS, sub-meter positioning accuracy could be obtained for source/receiver separation distances [19] [20] [31] with no restriction on the ranges. Further, absolute positioning on a geospatial grid could be determined to within $1 \mathrm{~m}$.

In order to transform the complex pressure data into its wavenumber representation, it is necessary to accurately measure the phase of the signal. Because the 


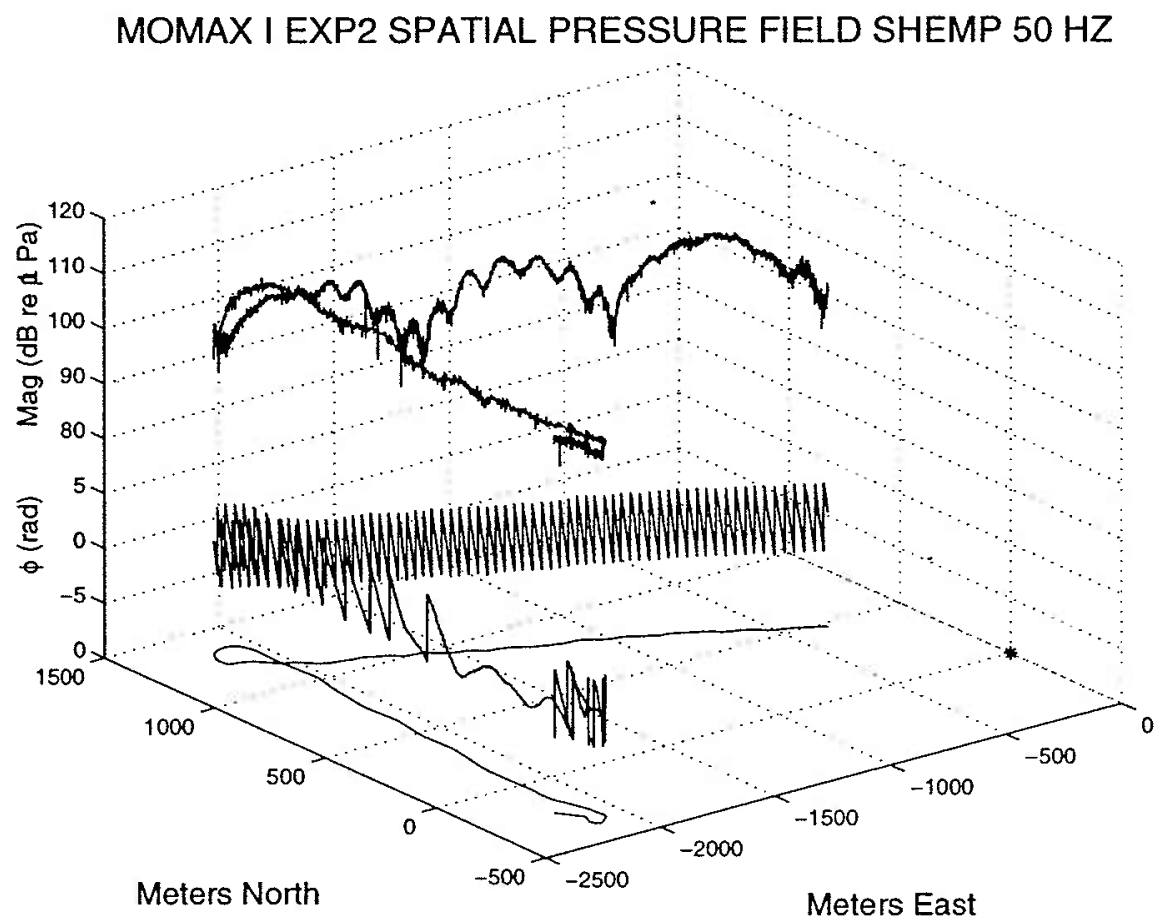

Figure 5-1: $50 \mathrm{~Hz}$ spatial pressure field measured with source located at the origin of the coordinate system. Top plot is pressure magnitude, center plot is phase, and bottom plot is drifter track geometry.

MOMAX approach is new, it is worthwhile to examine the phase of the acoustic signal in order to both comment on its quality and also possibly gain insight into the shallow water propagation problem. Figure 5-1 shows $50 \mathrm{~Hz}$ complex pressure measured on a two-dimensional grid relative to the source position. The effects of the source/receiver motion are clear in both the magnitude and phase plots as the receiver moves out in range from the source and then turns and moves broadside to the source. Referring back to the modal expressions for the pressure field given by equation (2.36) in chapter 2 , the phase of the complex pressure field measured in space is proportional to range. Using the radiation condition and the paraxial approximation for horizontal wavenumbers, given by

$$
k_{n}=k \sqrt{1-\epsilon_{n}} \approx k\left(1-\epsilon_{n} / 2\right) \text { for } \epsilon_{n} \ll 1,
$$


an approximate expression can be derived relating the time rate of change of the phase to the separation range rate between source and receiver [33],

$$
\frac{d r}{d t} \approx \frac{1}{k} \frac{d \phi}{d t}
$$

where $k$ is a typical wavenumber. The resulting expression can be, integrated in time to yield a relation between the phase and the source-receiver range,

$$
r=r_{o}+\frac{\phi}{k}
$$

where $r_{o}$ is the range from source to receiver at initial time $t_{o}$. From these relationships it is clear that as source/receiver separation distances increase, phase wraps with a positive slope, and wraps with a negative slope when distances are decreasing. These effects are evident in figure 5-2 where complex pressure is plotted as a function of time. The top two plots are complex pressure magnitude and phase, and the bottom plots are separation distance between source and receiver measured using GPS and calculated using equation (5.3) with initial range, $r_{o}$, assumed known as determined by a point GPS measurement at $t_{o}$. Of particular interest for this data set is how well the phase tracks the relative source/receiver positions through the turning point where the phase reverses itself when going from opening in range to closing in range. Figure 5-3 is a plot of range rates determined from both GPS measurements and phase measurements at $50 \mathrm{~Hz}$ using equation (5.2). From the figures, it is clear that there is good agreement between the measurements for source/receiver positioning using both GPS and acoustic phase. Using this type of analysis on data acquired using GPS navigated buoys thus shows great promise for doing source localization and tracking using measured phase data [58]. 

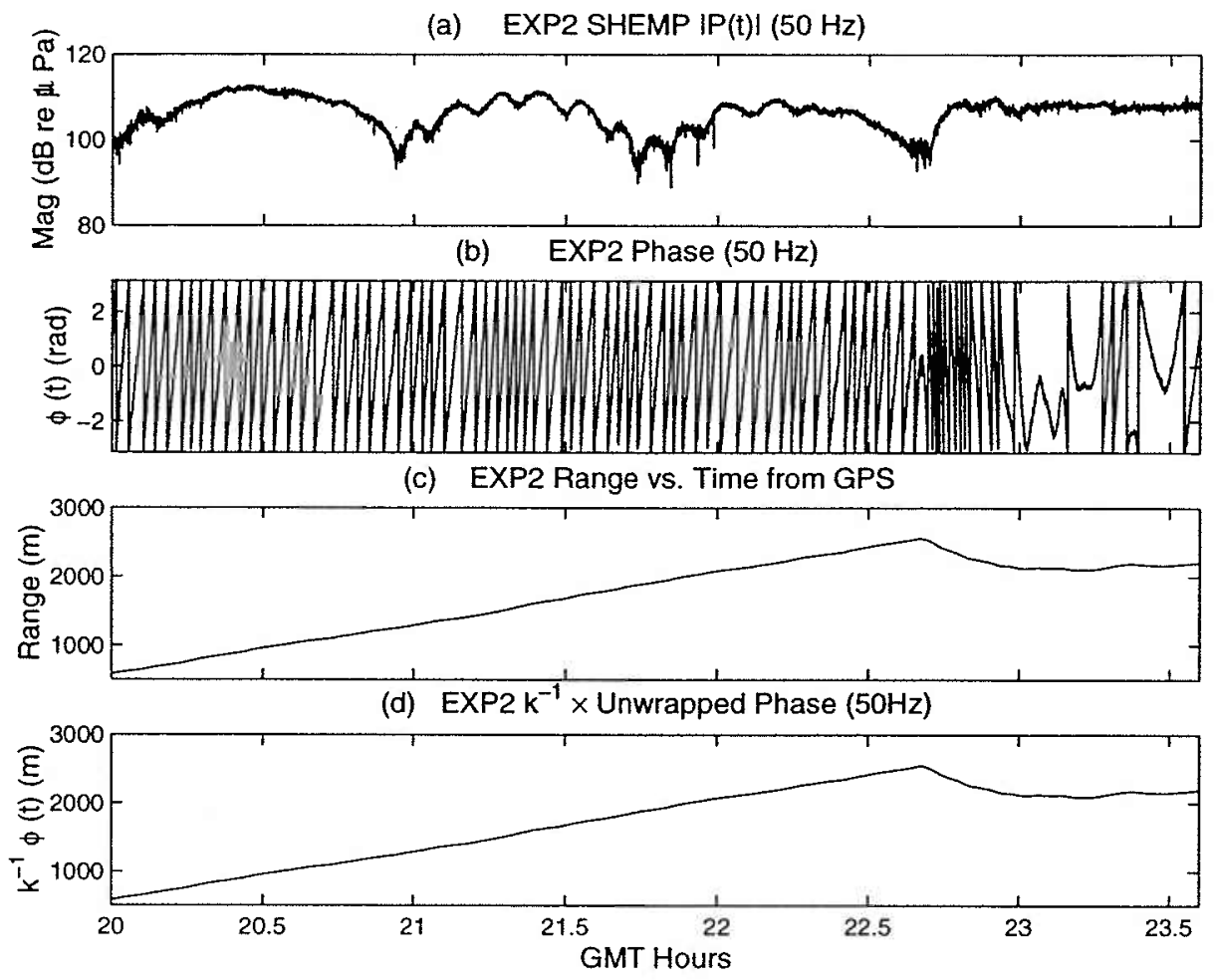

Figure 5-2: $50 \mathrm{~Hz}$ data plotted as a function of time with corresponding separation distance between source and receiver. Initial range for bottom plot determined from a single GPS measurement.

\subsection{Wavenumber analysis of MOMAX data}

\subsubsection{Geometrical interpretation}

Given the previous discussion of the complex pressure field measurements from the MOMAX experiments, it is of interest to look at results in the wavenumber domain. As discussed in chapter 2, Hankel transform based methods are to be used for examining horizontal wavenumber content of the propagating fields. Because these methods are based on a radial geometry, the measured two-dimensional spatial pressure data must be mapped to a radial grid. Of course, this is not ideal; it would be preferable to use the full two-dimensional inverse transform relationship given by (2.8), but the sparse nature of the measurements precludes its use. A further requirement of the Hankel transform based methods is that data analysis is limited to data where the source and receiver are either monotonically opening or closing in range. Given this 


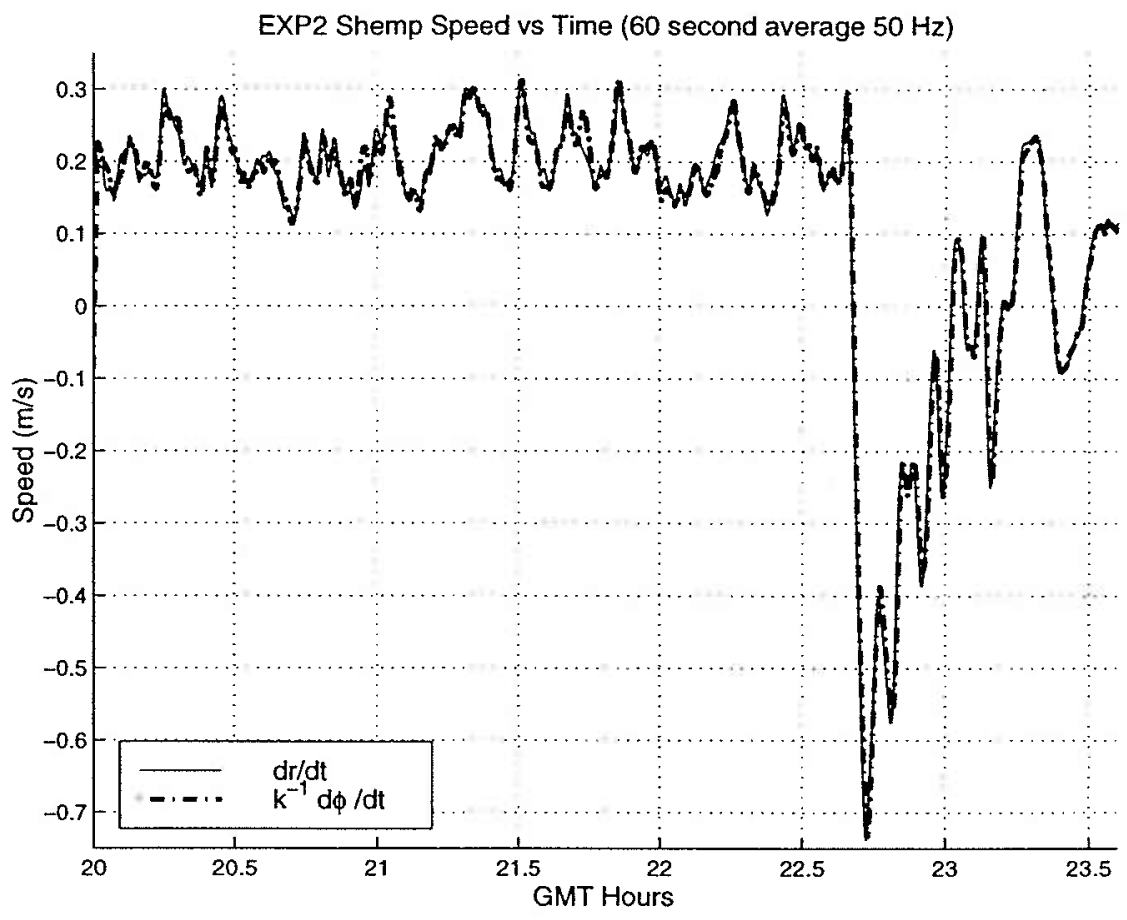

Figure 5-3: Source/receiver separation speeds determined from GPS and phase measurements of $50 \mathrm{~Hz}$ complex-pressure field.

requirement, spatial data must first be mapped as a function of range indicating the separation distance between source and receiver. From this mapping, data can be selected with sufficient range apertures for resolving individual modes. Referring to figure 5-2, data measured for GMT times between 20 and 22.6 hours is monotonically increasing with range and suitable for Hankel transform based processing. The method for mapping to a simple range grid from an arbitrary drift path is illustrated conceptually in figure 5-4. Having mapped the synthetic aperture data to a function of range relative to the source, other considerations must be given to interpretation of results given the full two-dimensional geometry of the source/receiver paths. Much like in the synthetic aperture experiments for linear arrays, assumptions are made as to the local nature of the measurements. Wavenumber content extracted for a given range interval is attributed to local waveguide properties for that range interval. Similarly, in this work, local waveguide properties are attributed to measurements made over the local 2-D spatial grid. Thus, local inversion results for a given sub-aperture 


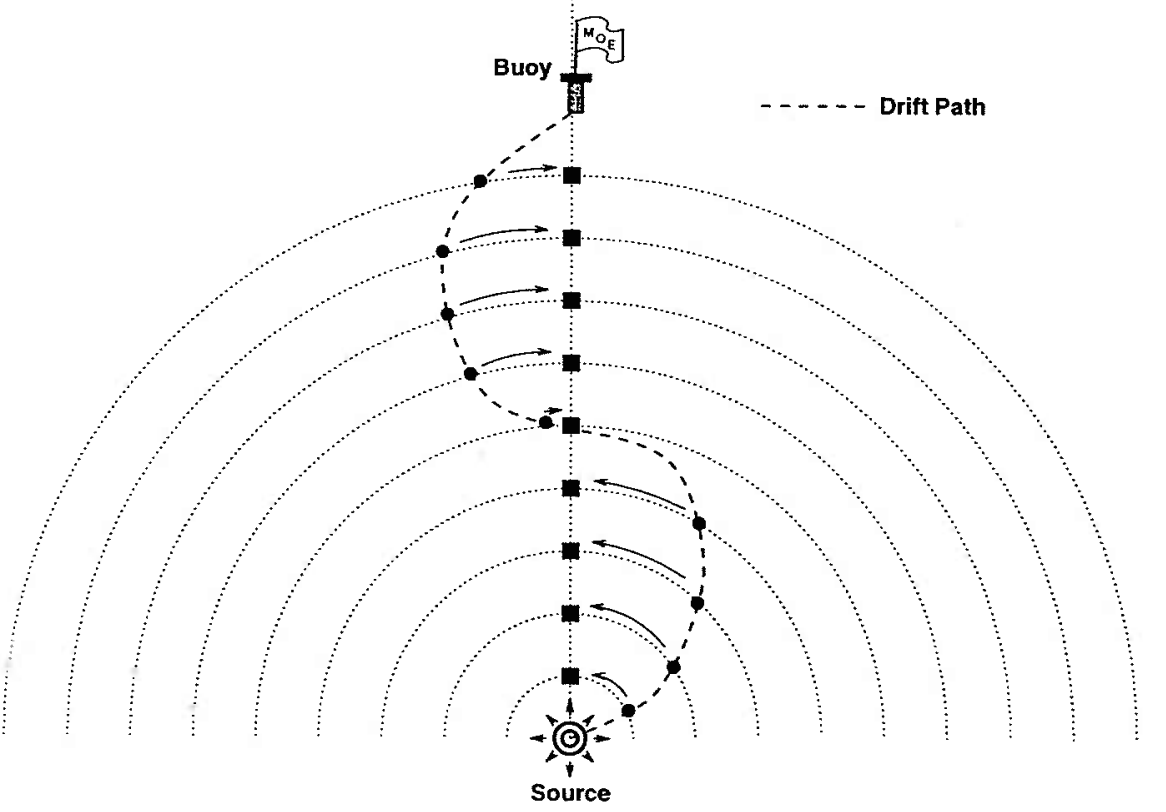

Figure 5-4: Schematic representing mapping of spatial pressure data to a range grid.

of data, must be given in terms of both range and bearing relative to the source. Although not much more will be said regarding this issue for the results that follow, it becomes an issue when synthesizing results from multiple experiments in a particular region. Additionally, when mapping the data to the range grid, consideration must be given to the projection angle of the data onto the local radial. Depending on the nature of the spatial variability of the waveguide, data which crosses many lines of azimuth, while having a monotonic range separation, may have to be interpreted differently then data which is taken along a constant bearing. An example would be the case where the bathymetry approximates a wedge. In this case the interpretation of wavenumber evolution depends on the orientation of the receiver track to the source. For a track where the receiver travels perpendicular to lines of constant bathymetry, wavenumber evolution can be attributed solely to range-dependence of the environment. If the receiver track slowly spirals around the source, wavenumber evolution would be due to both azimuthal- and range-dependent changes in the environment. For this thesis work, data are considered for cases where the source/receiver geometry is dominated by their separation in range with limited changes in relative bearing. 
As such, when mapping the data to a range-grid, azimuthal symmetry is assumed over the apertures considered. In future work, as more of the data are analyzed, including more complicated drift paths, more sophisticated techniques may have to be employed to exploit the full spatial measure of the complex pressure field. One area to explore would be the projection theorems commonly used in image processing. As discussed by Lewitt [46], these theorems relate a one-dimensional transform along a path to the full two-dimensional transform of a region that contains the path. Included among the transform theorems is the projection-slice theorem [46] that relates a one-dimensional Fourier transform along a path to the full two-dimensional Fourier transform. This may be a way to exploit the sparse nature of the MOMAX 2-D pressure field measurements to extract the full spatial evolution of the wavenumber spectra.

\subsubsection{Modal Mapping - Single Mode}

During SWAT 01 measurements were made using a $20 \mathrm{~Hz}$ source, corresponding to a wavelength of about $75 \mathrm{~m}$. For the experimental water depths of approximately 70 $\mathrm{m}$, and a sandy bottom with sound speed $1600 \mathrm{~m} / \mathrm{s}$, theory predicts that only a single mode would be excited for a Pekeris waveguide [28]. Figure 5-5 shows the source and receiver tracks for the $20 \mathrm{~Hz}$ experiment, where the source location is indicated by the location of the R/V Endeavor. 


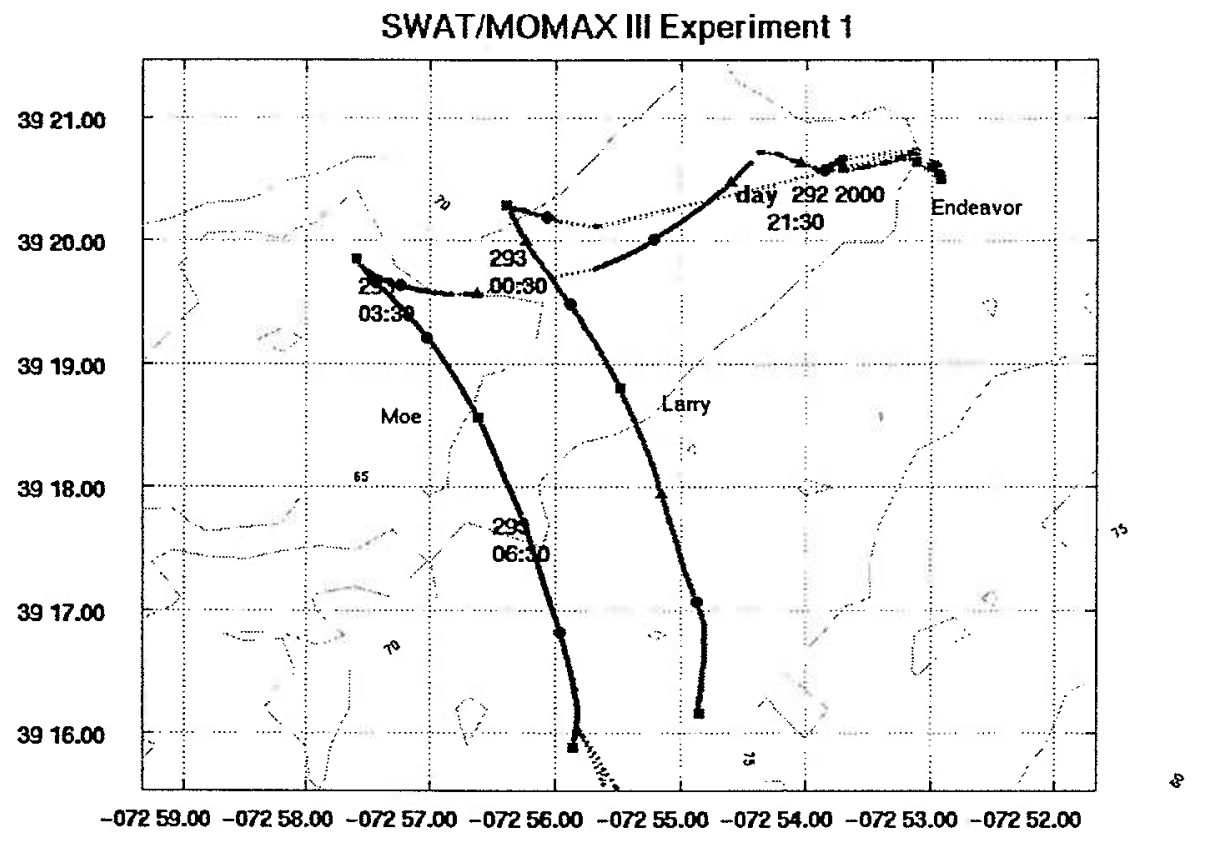

Figure 5-5: Source/Receiver geometry for SWAT $20 \mathrm{~Hz}$ experiment.

Data are considered near the end of the experiment run when the source is approximately stationary and the buoys are between 7 and $9 \mathrm{~km}$ from the source and drifting in a southerly direction.

The single mode case is of interest because the modal representation of the pressure field is given by a single term. Using the adiabatic approximation for a rangedependent waveguide, modal evolution with range can be determined without transforming to the wavenumber domain. The adiabatic mode expression for the pressure field given a single mode is written,

$$
P=A e^{i \Phi}=A_{1} \frac{\exp \left(i \int_{0}^{r} k_{1}\left(r^{\prime}\right) d r^{\prime}\right)}{\sqrt{\int_{0}^{r} k_{1}\left(r^{\prime}\right) d r^{\prime}}}
$$

where,

$$
A=\frac{A_{1}}{\sqrt{\int_{0}^{r} k_{1}\left(r^{\prime}\right) d r^{\prime}}}, \quad \Phi=\int_{0}^{r} k_{1}\left(r^{\prime}\right) d r^{\prime} .
$$

Examining the phase term, $\Phi$, modal evolution of the single mode, $k_{1}(r)$, can be 
determined directly by,

$$
k_{1}(r)=\frac{d \Phi}{d r}
$$

This method was applied to the $20 \mathrm{~Hz}$ data measured on the buoys Moe and Larry for SWAT experiment 1 as described. Pressure magnitude and phase measured on Larry are shown in figure 5-6. The top magnitude plot does not contain the deep nulls typical of modal interference suggesting the interaction of several modes. Although the magnitude plot appears somewhat noisy, the bottom plot shows the phase to be very well behaved. Unwrapping the phase data and applying a 50 point moving average filter, $k_{1}(r)$ was determined using equation (5.6) as shown in figure 5-7. Also shown are the results for a $500 \mathrm{~m}$ aperture sliding window AR transform. Model order selection for this and other analysis on real data was based on $N / 3$ where $N$ is the number of data samples. The number of points for discretization in wavenumber space was 8192 , which gives a bin size of $7.6699 \mathrm{e}-04 \mathrm{~m}^{-1}$, which is greater than the variance of the estimator determined by the method of Sakai which for a data aperture of $500 \mathrm{~m}$ and an assumed SNR of $10 \mathrm{~dB}$, is approximately $1.25 \mathrm{e}-4 \mathrm{~m}^{-1}$. However, even given the low resolution in wavenumber space for the AR estimator, along with low resolution in range due to the finite step side of the estimator, good agreement is shown between the two methods. The analysis was repeated for the data measured on Moe with similar results as shown in figures 5-8 and 5-9. The sliding window transform results for MOE are not as good at the middle ranges because there was a dropout in the data around 7900 meters that affects the AR wavenumber estimates as the transform window overlaps the region of missing data. 

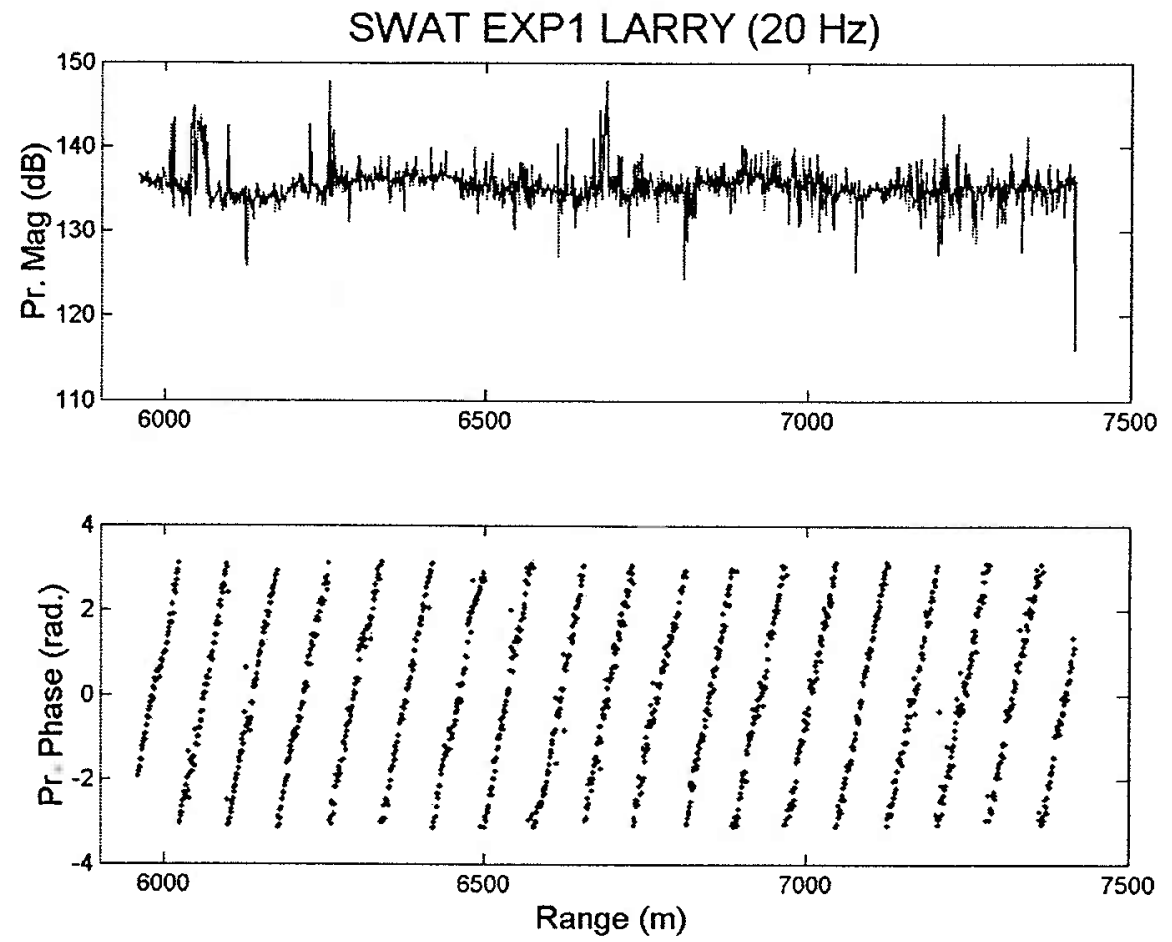

Figure 5-6: $20 \mathrm{~Hz}$ Complex pressure measured on Larry.

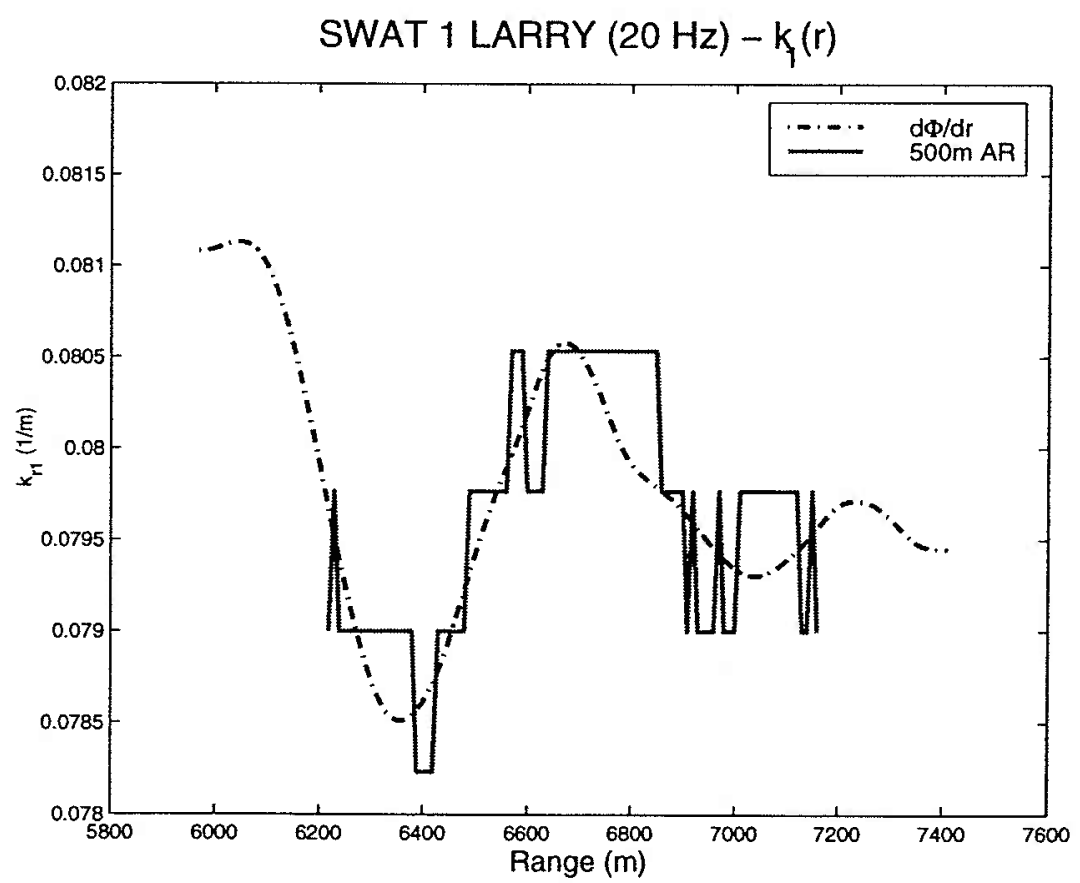

Figure 5-7: Modal evolution for single mode at $20 \mathrm{~Hz}$ on Larry. 
SWAT EXP1 MOE $(20 \mathrm{~Hz})$
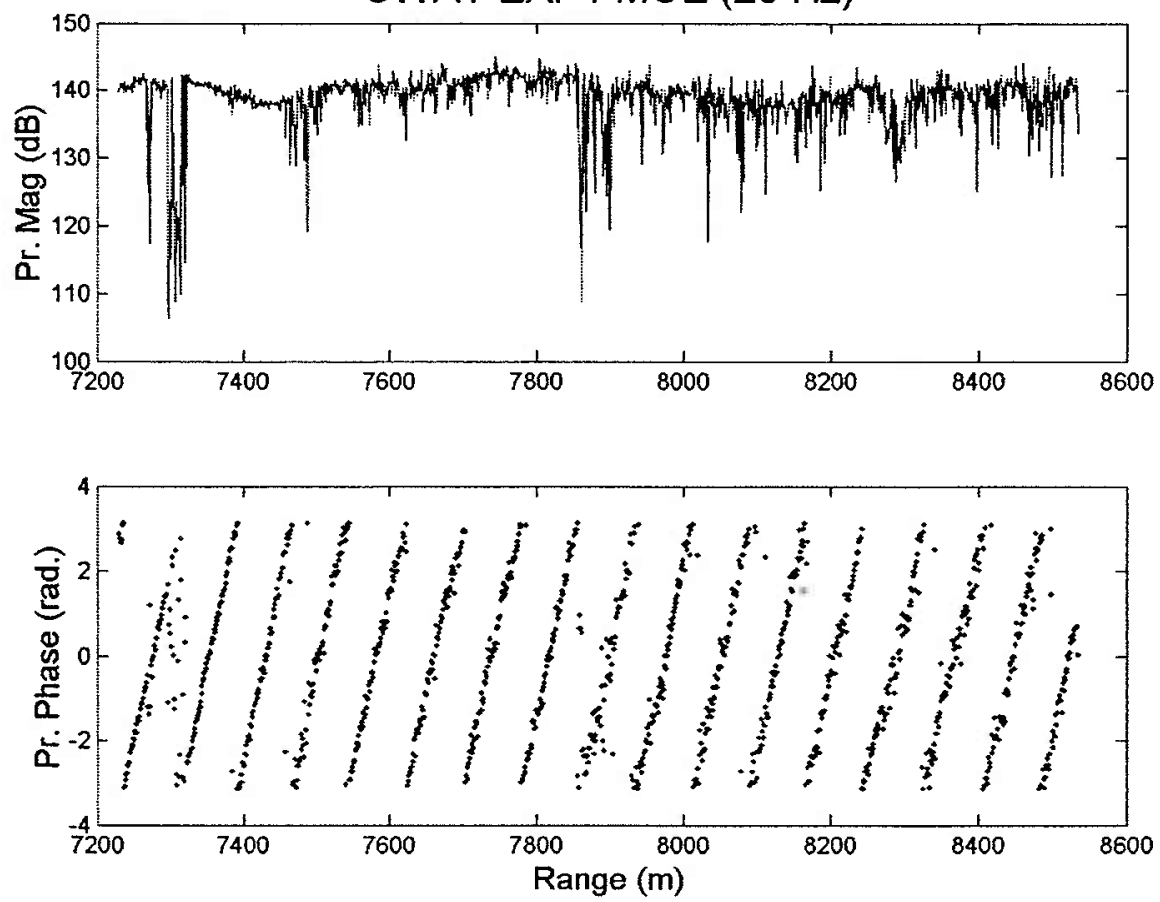

Figure 5-8: $20 \mathrm{~Hz}$ Complex pressure measured on Moe.

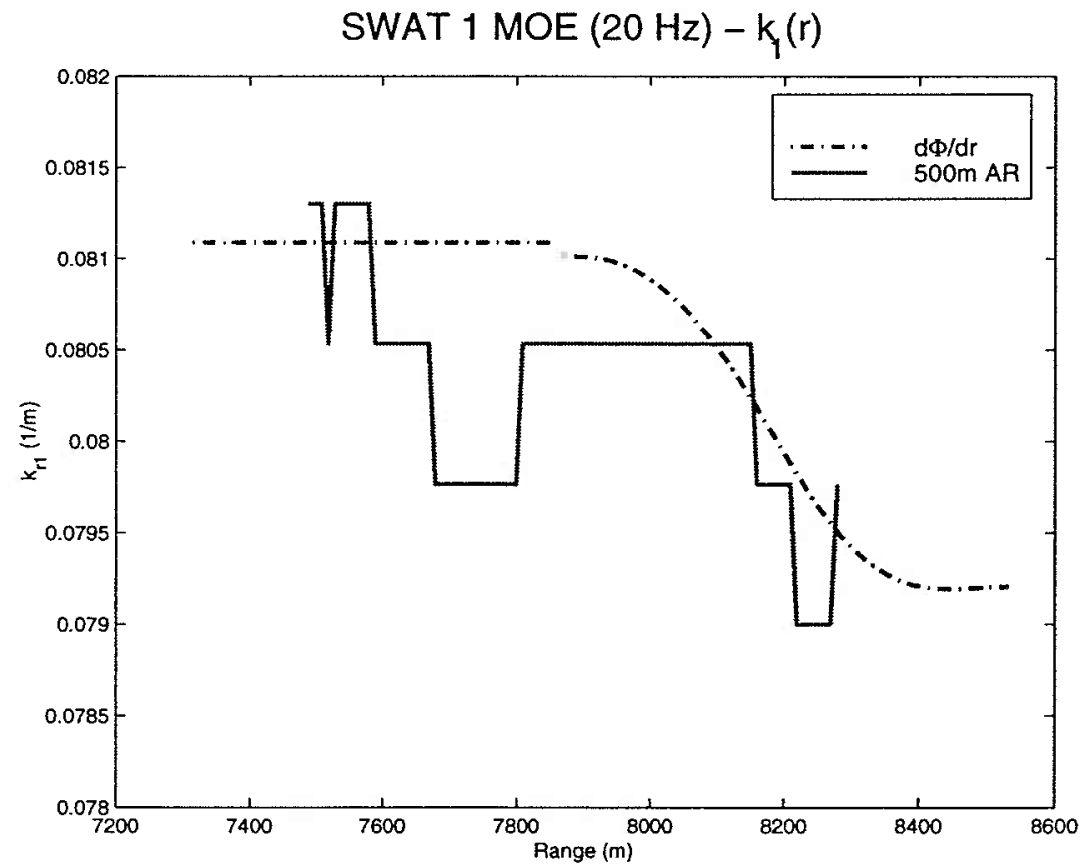

Figure 5-9: Modal evolution for single mode at $20 \mathrm{~Hz}$ on Moe. 


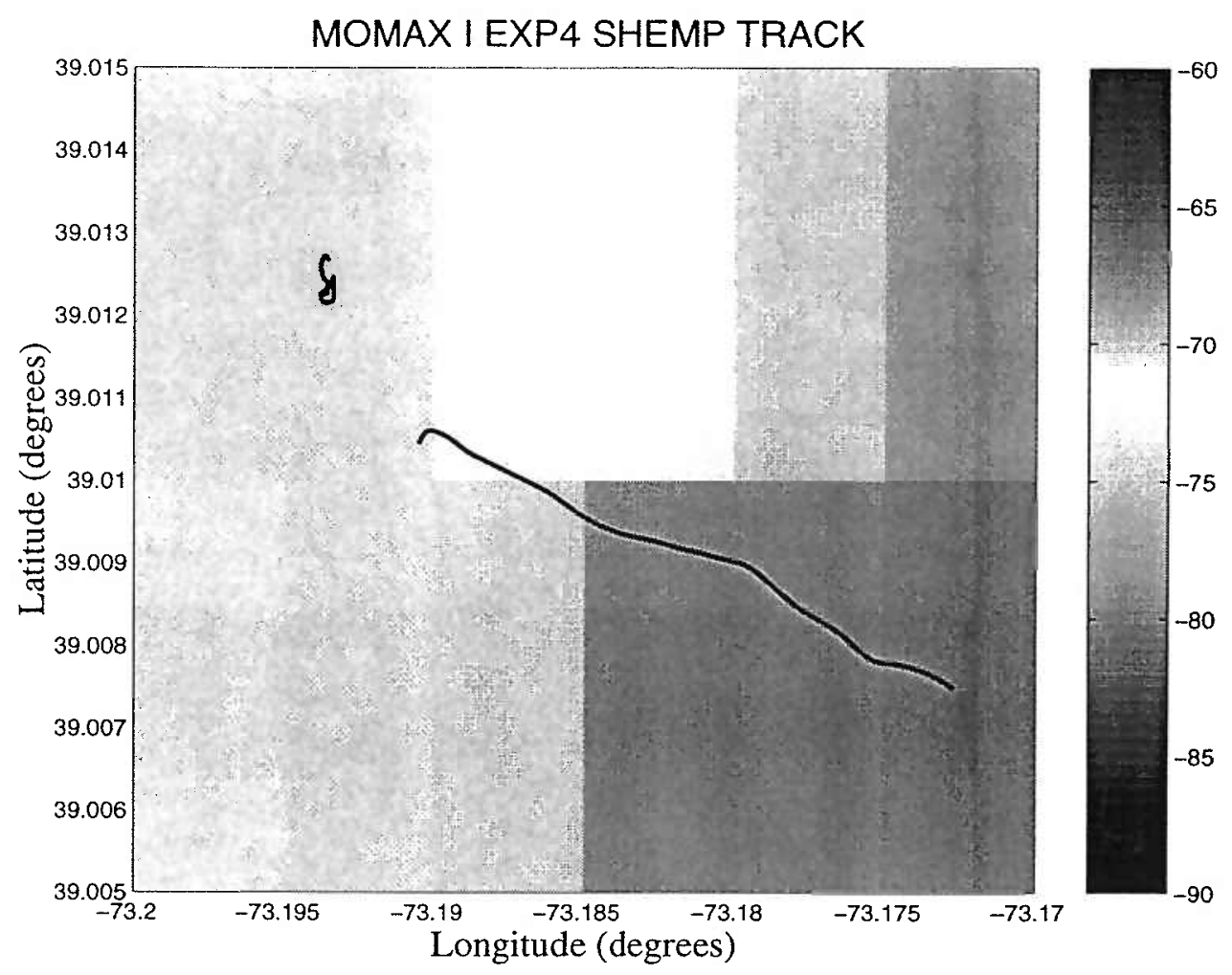

Figure 5-10: MOMAX I experiment 4 geometry and bathymetry. Depth is indicated by the colorbar on the right in meters. For this experiment, the buoy moved very little as indicated by the track at the upper left, and the source was moving slowly along a constant bearing.

\subsection{Modal Mapping - Perturbative Inversion Re- sults}

In this section results are presented where the input data were eigenvalues determined from the MOMAX measured pressure fields. Range independent results are presented for two different experiments with different source/receiver geometries. For each case, eigenvalues are determined and used as input data to a perturbative inverse algorithm to determine an effective sound speed profile for the sediment. Magnitude plots of the measured data are compared with model runs using the profile resulting from the inversion. The experiments considered are from the MOMAX 97 data set measured in the STRATAFORM region near New Jersey.

Figure 5-10 shows the source/receiver configuration for MOMAX 97 experiment 


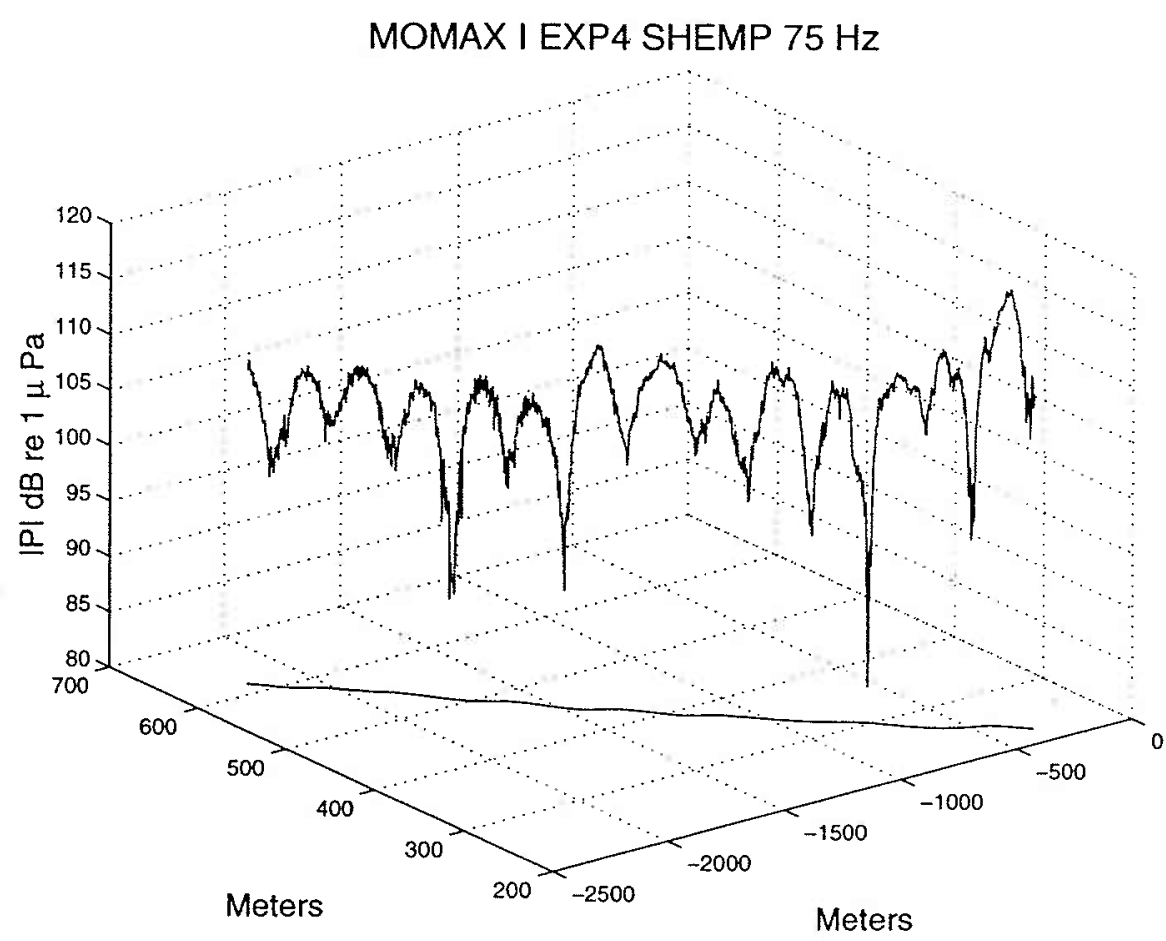

Figure 5-11: $75 \mathrm{~Hz}$ pressure field magnitude for MOMAX 97 experiment 4 plotted over the drift track. Drift track is plotted relative to source position.

4. The bathymetry for the area indicated an average depth of about 77 meters. For this experiment, the receiver is considered stationary and the source is closing in range roughly along a radial. For this geometry it is straightforward to apply the Hankel transform based methods to extract wavenumber content. The magnitude of the pressure field at $75 \mathrm{~Hz}$ for this experiment is shown in figure 5-11 in 3-D coordinates. The complex pressure as a function of range is shown in figure 5-12 where phase is shown to wrap with range. The aperture for this experiment was only about $1300 \mathrm{~m}$ so a single transform was taken to determine horizontal wavenumber content. The spectrum resulting from a classical spectral estimator using Matlab's psd function [60] indicated 5 dominant propagating modes. The resulting spectrum is shown in 5-13 where the result was interpolated onto a finer wavenumber spacing grid for determining the exact peak locations. Using the eigenvalues determined from the peak locations of the spectrum, a background model was determined for initializing a perturbative inverse algorithm to determine compressional wave speed in the 

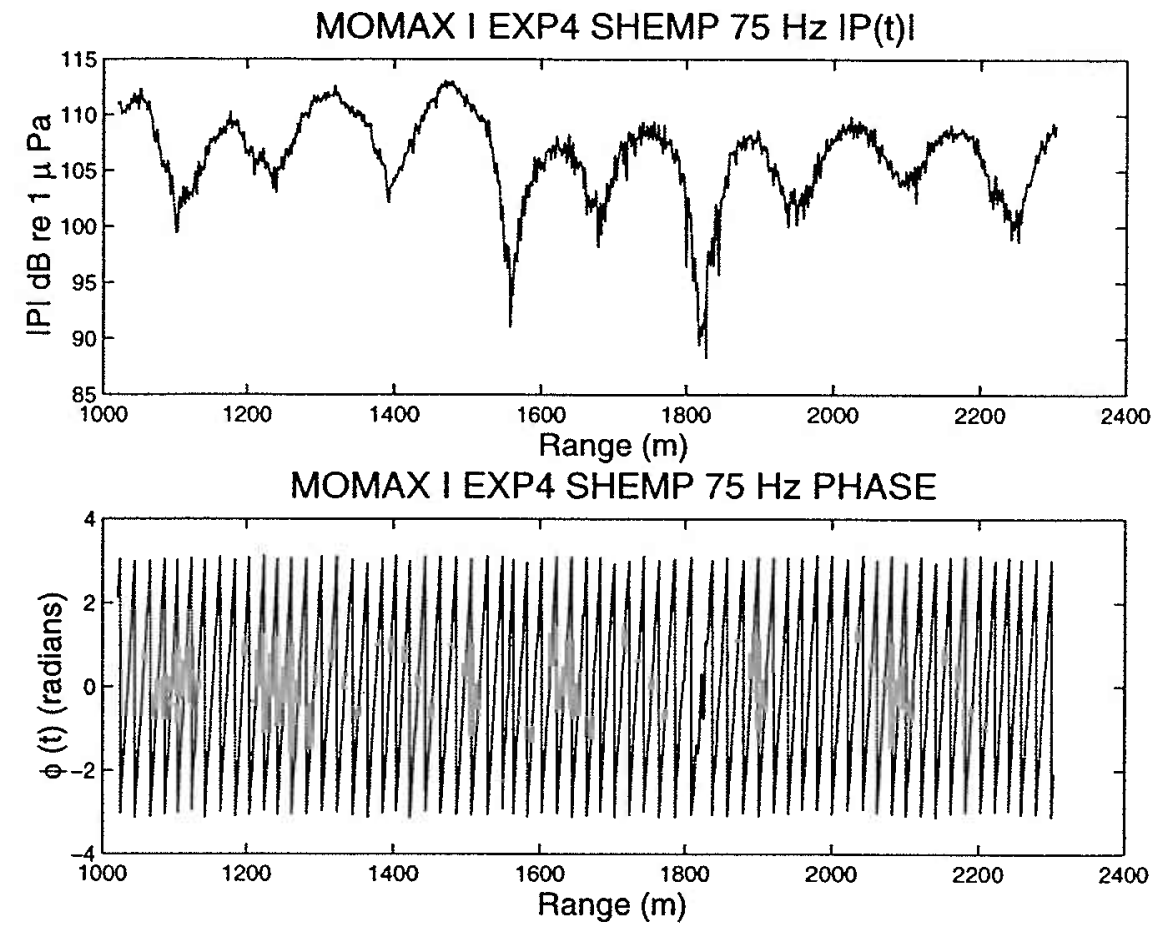

Figure 5-12: $75 \mathrm{~Hz}$ complex pressure plotted as a function of range for MOMAX 97 experiment 4 .

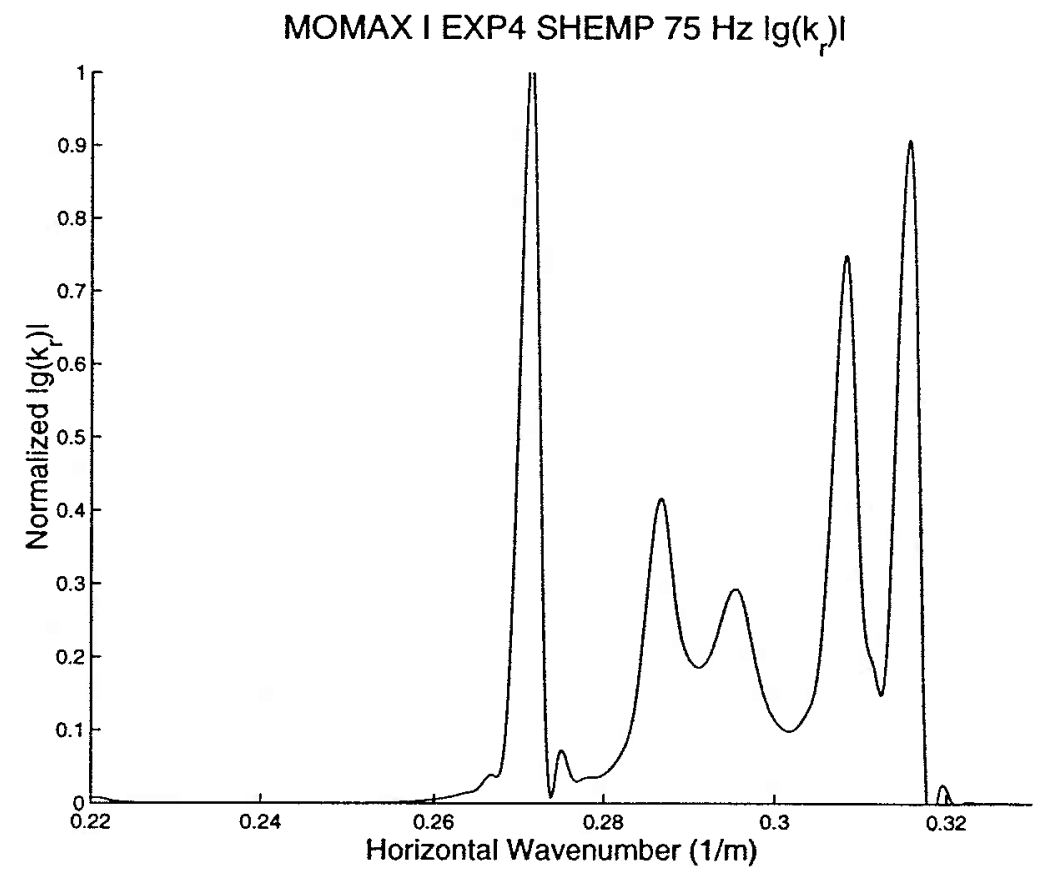

Figure 5-13: Horizontal Wavenumber Spectrum for full aperture $75 \mathrm{~Hz}$ data. 


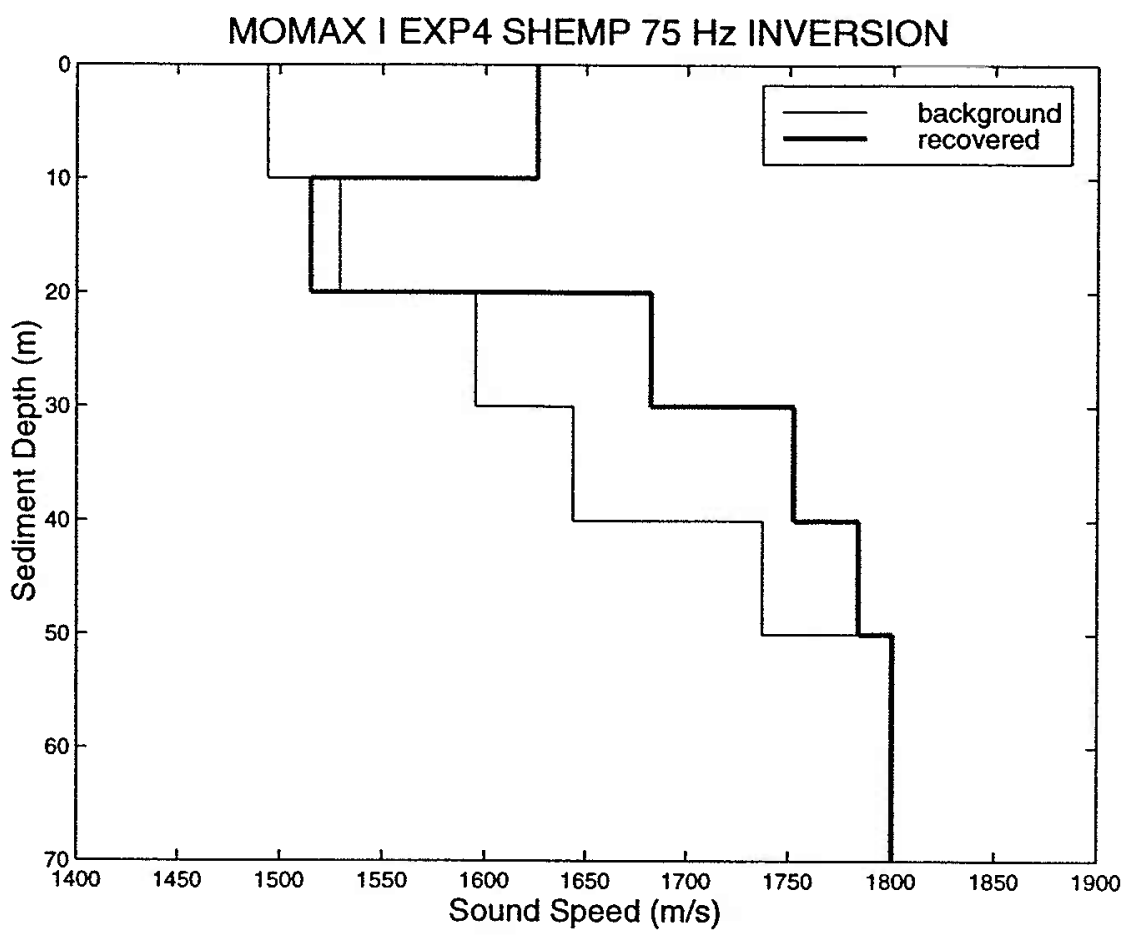

Figure 5-14: Starting profile and inferred profile for MOMAX 97 experiment 4. Density was $1.8 \mathrm{~g} / \mathrm{cm}^{3}$ and no attenuation was used.

sediment. The model was determined using layers with sound speeds corresponding to the phase speed of each propagating mode. Given a number of layers and sound speeds, The basement depth and corresponding sound speed, are adjusted by trial and error until the number of eigenvalues for the model matches the number measured in the data. As the basement depth is increased or decreased, each layer is stretched or compressed accordingly. KRAKEN was used to determine the model eigenvalues for the background model. Using the perturbation algorithm described in chapter 2, the background model was updated iteratively until convergence was achieved between the eigenvalues of the updated model and the measured values. The background starting model is plotted along with the inferred sound speed profile after 30 iterations in figure $5-14$. The density in the sediment was was $1.8 \mathrm{~g} / \mathrm{cm}^{3}$ with no attenuation in the model. The inferred sound speed values between 1500 and $1800 \mathrm{~m} / \mathrm{s}$ for the upper 50 meters of sediment are consistent with sound speeds for sand and silty-sand [36] as expected for the STRATAFORM region. Using the 


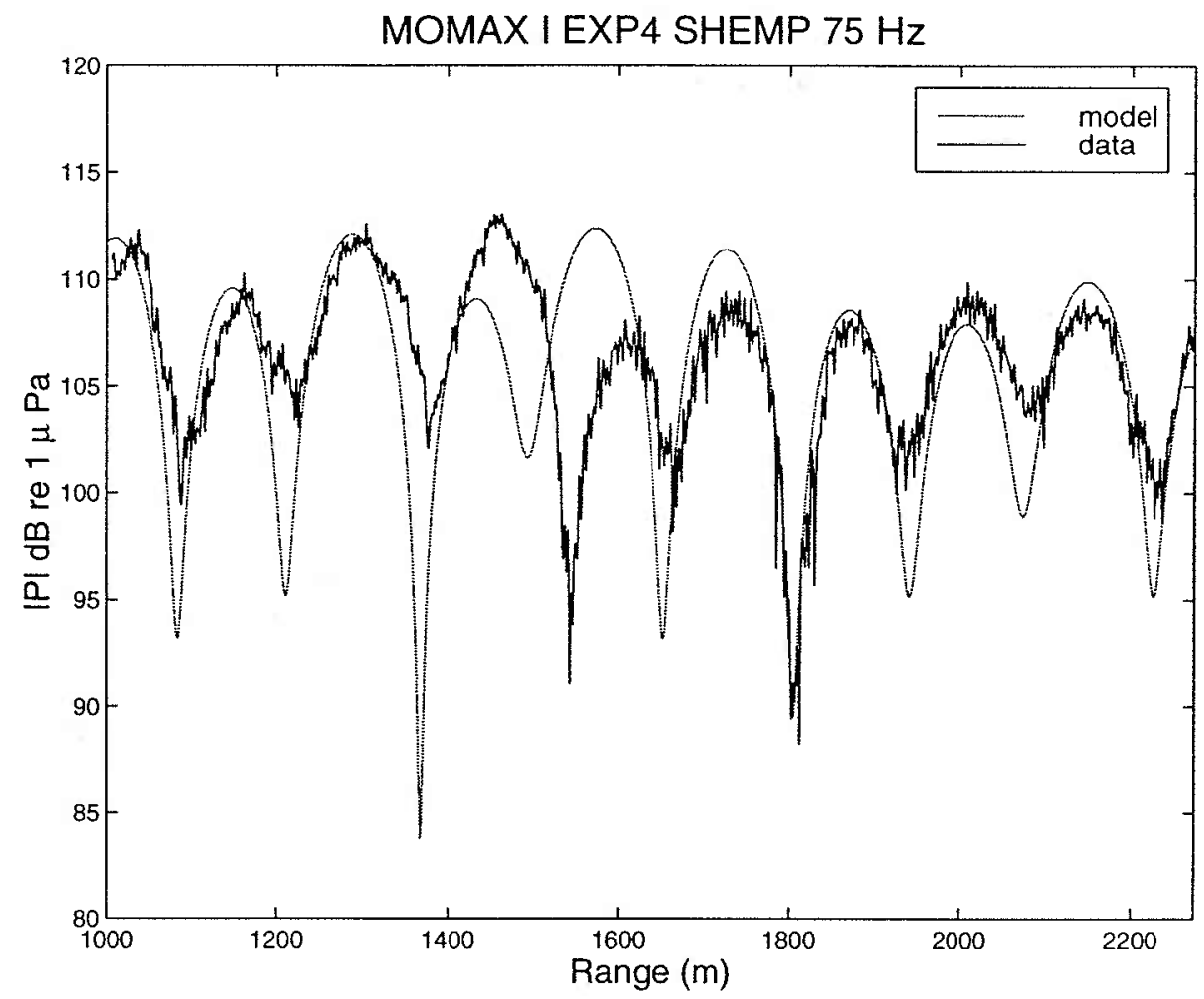

Figure 5-15: Comparison of $75 \mathrm{~Hz}$ measured and synthetic pressure magnitude data for inversion of MOMAX 97 experiment 4 .

inferred sound speed model for the sediment, along with measured sound speed values for the water column, range-independent synthetic pressure data were generated and compared to the measured data as shown in figure 5-15. The inferred model predicts the observed modal interference pattern well with the exception of the region around 1500 meters. This suggests that a range dependent inversion might be more suitable for this region. 


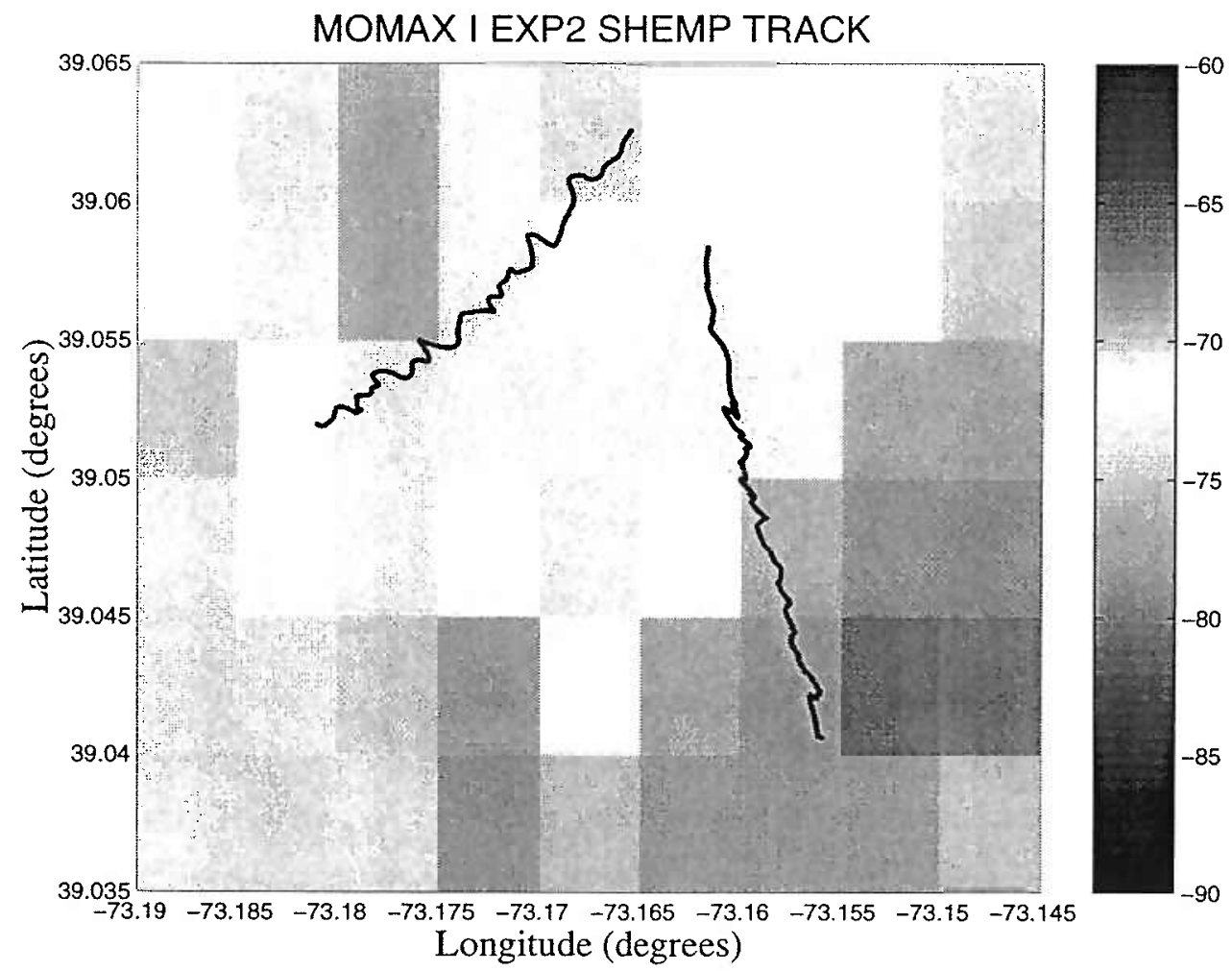

Figure 5-16: MOMAX 97 experiment 2 geometry and bathymetry. Buoy track is shown in upper left and source track in bottom right. Water depth is indicated by the colorbar on the right in meters.

The source/receiver geometry and bathymetry for a second experiment from MOMAX 97 is shown in figure 5-16. For this experiment both the source and receiver were drifting independently along different paths. This geometry is a representation of that described by Schmidt and Kuperman [73] where the source and receiver are allowed to move at different angles relative to one another in the same horizontal plane. For the case where the angles between the velocity vectors of the source and receiver remain constant, the radial vector between the source and receiver can be considered constant and the data mapped to a radial grid for application of Hankel transform based methods. The data represented on a grid with its origin located at the source is shown in figure 5-17. These data are easily mapped to a range grid and the full aperture spectrum estimate is shown in figure 5-18.

The spectrum determined using the AR spectral estimator showed contributions from 6 dominant modes whose eigenvalues where used as input data to determine a 


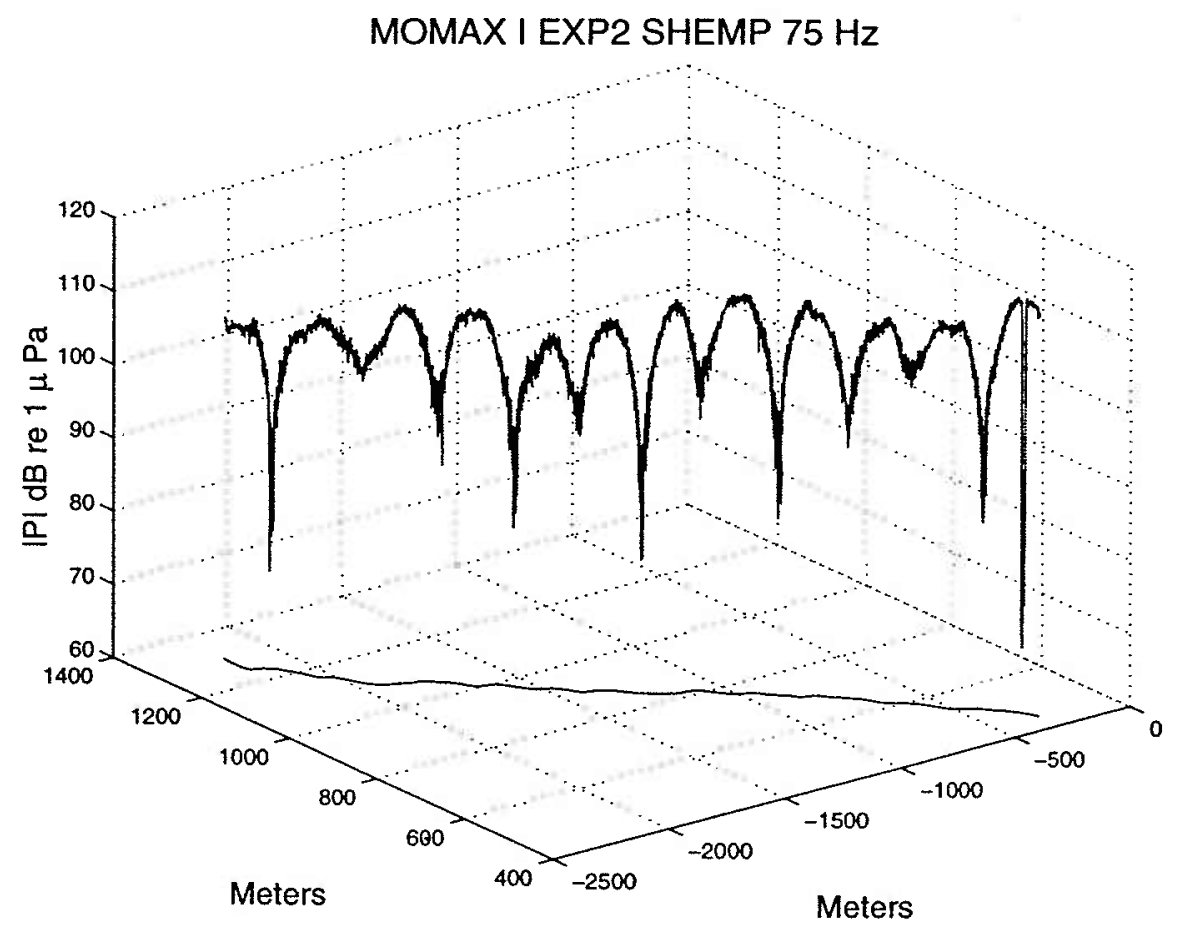

Figure 5-17: $75 \mathrm{~Hz}$ pressure field magnitude for MOMAX 97 experiment 2 plotted. over the drift track. Drift track is plotted relative to source position.

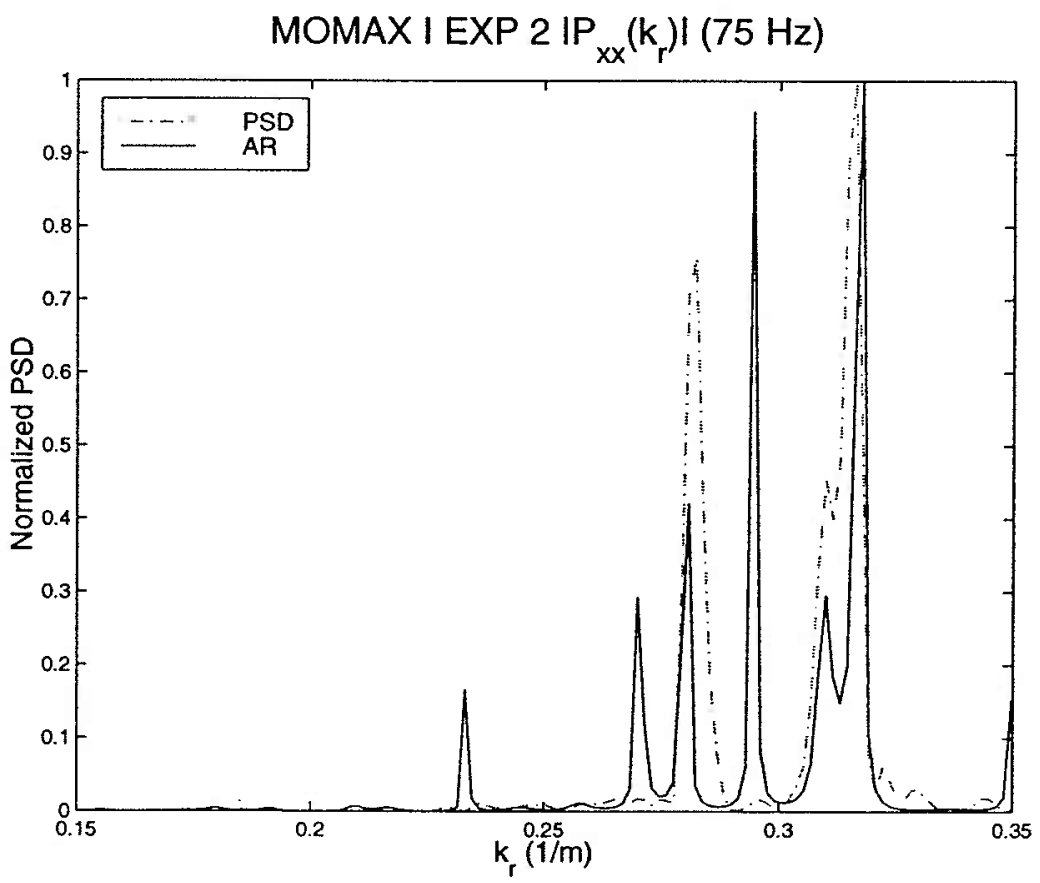

Figure 5-18: Horizontal wavenumber spectrum estimate for MOMAX 97 experiment 2 data at $75 \mathrm{~Hz}$. AR estimate is solid line, PSD estimate is dashed. 
background starting profile for perturbative inversion. An estimate of the spectrum for a the classical estimator with a Hanning window applied to the data is shown for comparison. Of particular significance is the lack of any peak in the classical estimate at a wavenumber around $0.295 \mathrm{~m}^{-1}$. Initial inversion work using wavenumber values from the dominant peaks in the classical spectrum gave non-physical results. It also serves to illustrate the subjective nature of picking which peaks are significant in the spectral plots for determination of wavenumbers for construction of background model. Using the AR results, the background sound speed profile and the resulting inferred profile are shown in figure 5-19. The inferred profile shows more resolution because the layers in the starting profile were split in half and the smoothness constraint applied to the least squares problem of the perturbation algorithm. This step represents some fine tuning to the general inverse algorithm and was performed with guidance from Dr. Subramaniam Rajan [68]. Using the inferred sound speed profile, synthetic data were generated using KRAKEN and compared to the measured data as shown in figure $5-20$.

The model/data comparison is in general very good with the modal interference pattern matching very well. However, in order to match the model to the data, the ranges for the data had to be shifted by 370 meters. It is unclear if this shift was necessary to accommodate the geometry of the source/receiver motion or if there were errors made in the measurements. One difficulty of the moving source and receiver problem is in locating the result of the inversion geographically. For the moving source/receiver problem it is not possible to identify a specific region of the waveguide from which the local wavenumber measurement results. This would be particularly difficult in an environment that has properties that vary in both range and azimuth. From the analysis of Hawker [38] and Schmidt and Kuperman [73], it is also clear that the source/receiver motion will impart a shift in the horizontal wavenumbers of the the spectrum relative to that of a stationary experiment. The shifted wavenumbers are what is measured and the inversion is based on using the 


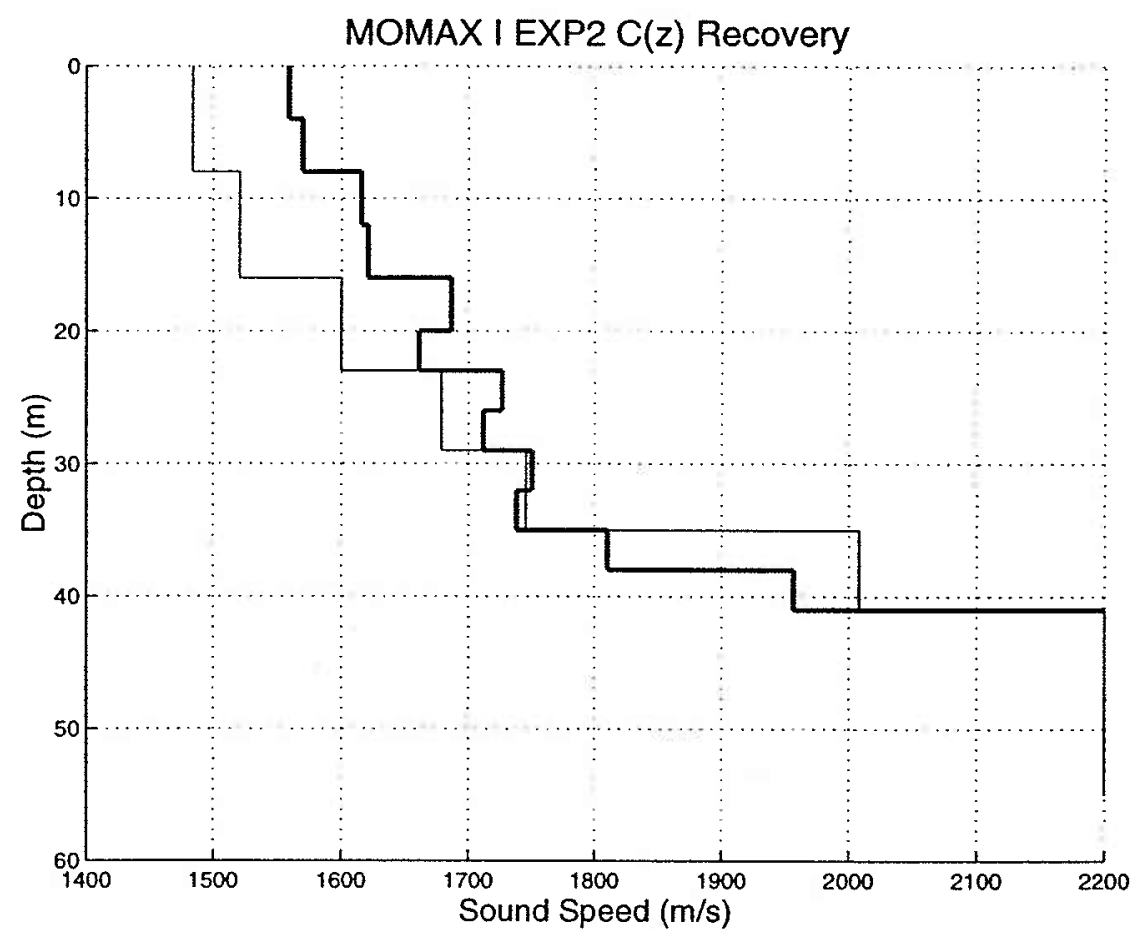

Figure 5-19: Starting profile (thin) and inferred (thick) sediment sound speed profile for MOMAX 97 experiment 2. Density was $1.8 \mathrm{~g} / \mathrm{cm}^{3}$ with no attenuation used.

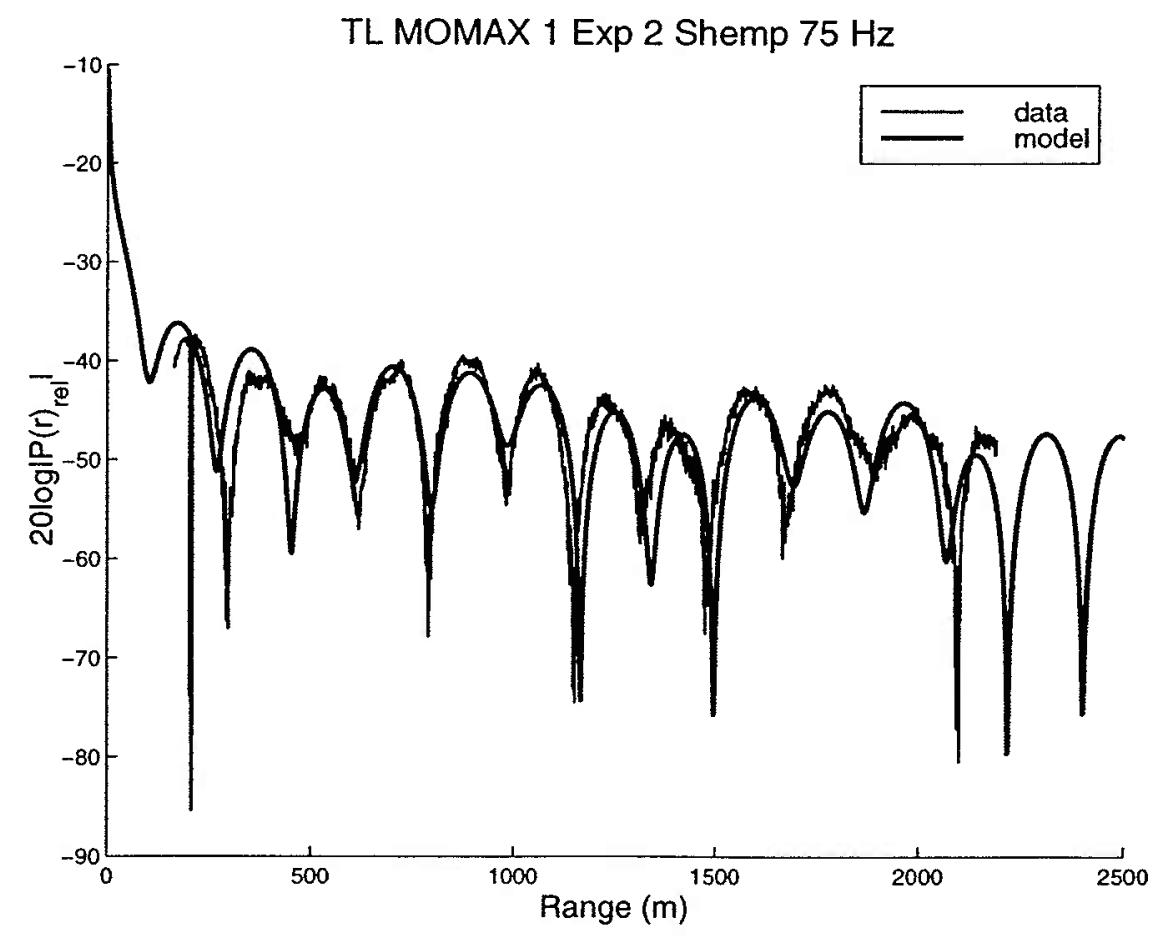

Figure 5-20: Comparison of measured and synthetic data for inversion of MOMAX 97 experiment 2. 
shifted eigenvalues. For the low velocities associated with the drifting source and receiver in this experiment the expected wavenumber shift should be negligible. This is confirmed by the good agreement between the measured and modeled pressure field magnitude shown in figure 5-20 that indicates a good match between the eigenvalues of the inferred geoacoustic model and the measured eigenvalues. However, these results also suggest that it is only necessary to match the eigenvalues of the propagating modes, and not necessarily the propagation environment, to generate a field that matches the measured data. Thus, when determining the geoacoustic model for a given environment, it may be necessary to shift the measured modal eigenvalues to account for source/receiver geometry and motion in order to infer the true geoacoustic properties of the seabed. 


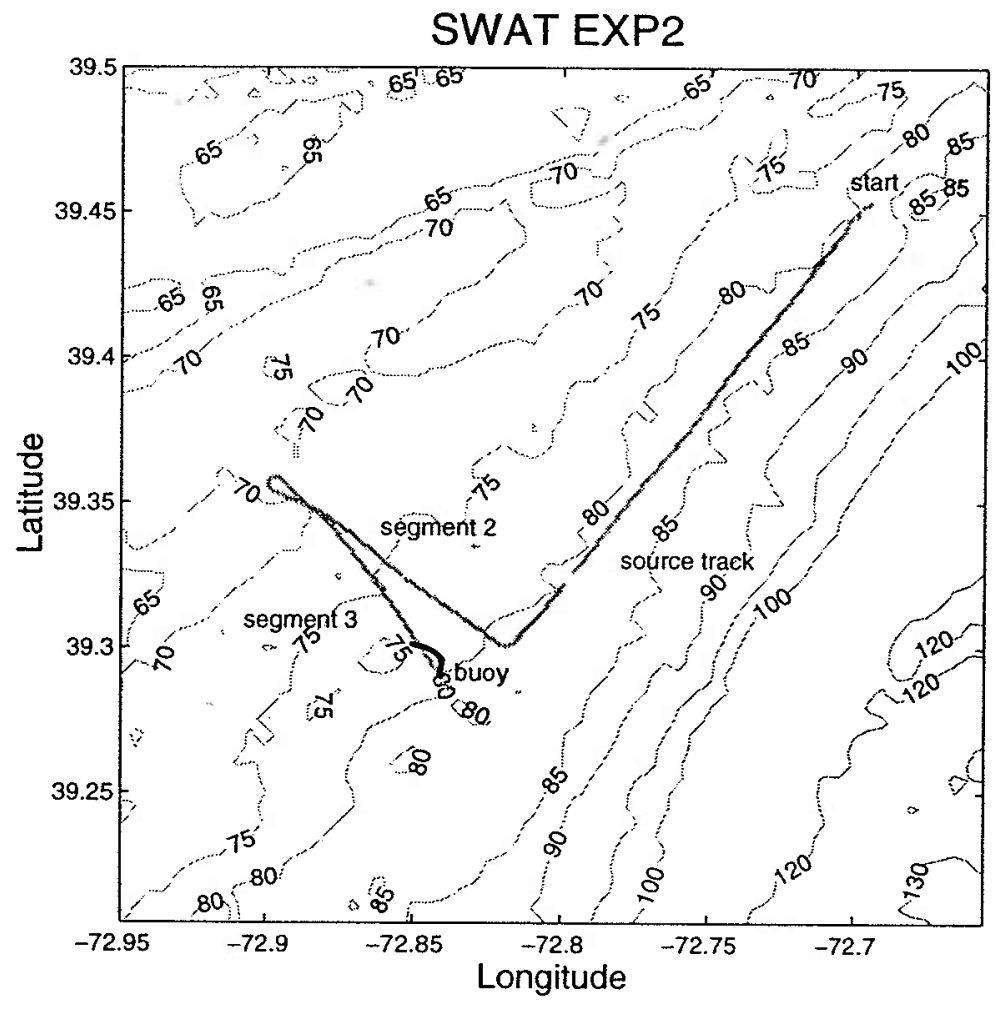

Figure 5-21: Source receiver geometry for SWAT experiment 2 with region of source track doubling back on itself. Segment 2 indicates time when source is moving away from buoy and segment 3 indicated time when source is closing on buoy.

\subsection{Modal Mapping - Moving Source Analysis}

In the preceding section, a discussion was given for the moving source/receiver problem when the source and receiver move along different tracks but very slowly. In this section, the problem is discussed for a moving source that traverses the same space in the waveguide twice. In chapter 2, the results of Hawker [38] and also Schmidt and Kuperman [73] showed that a shift in the phase of the complex pressure field could be expressed as a shift in the horizontal wavenumbers for a source moving at a constant velocity where the shift is proportional to modal group velocity. This phenomenon was observed during the SWAT experiment when wavenumber estimates made for a source moving out and back over the same waveguide track were found to be shifted relative to one another. The experimental geometry for this experiment is shown in figure 5-21 where the source goes out along a radial in range and then comes back 


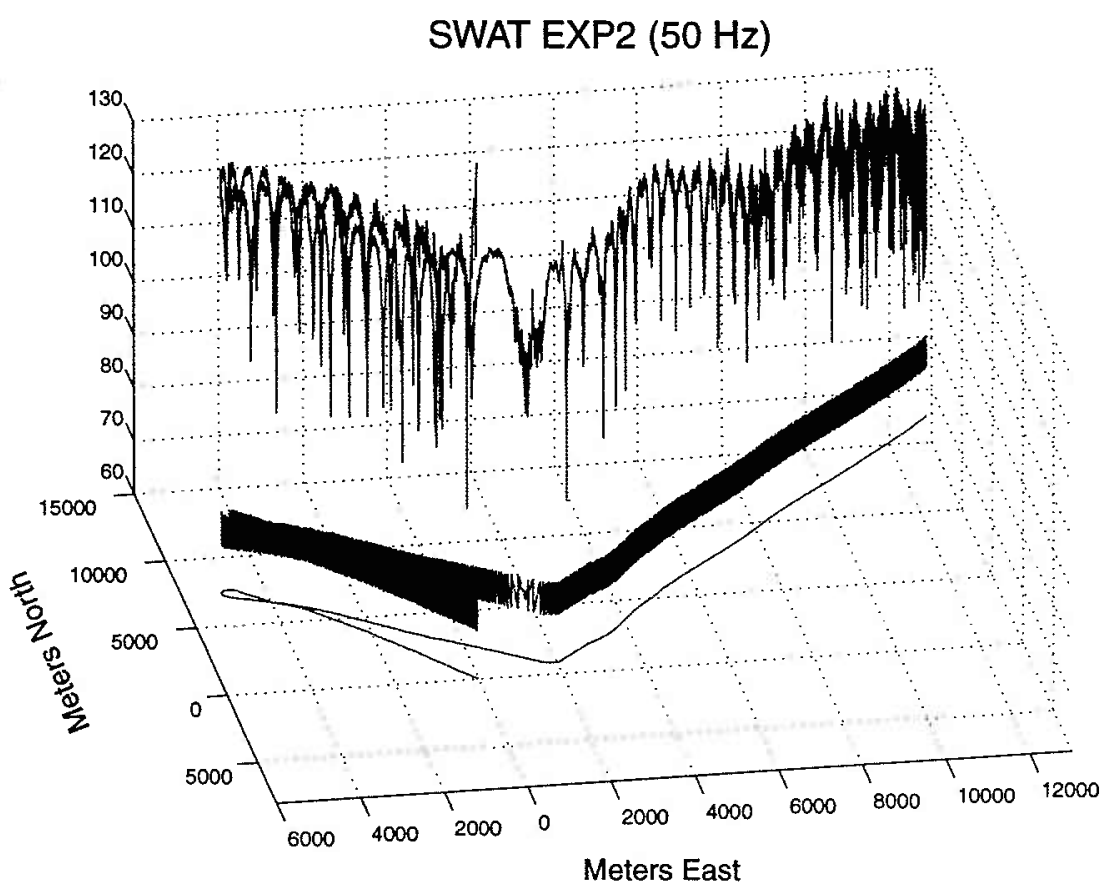

Figure 5-22: $50 \mathrm{~Hz}$ pressure field for SWAT experiment 2. Source is moving at a constant speed of $2 \mathrm{~m} / \mathrm{s}$.

again along roughly the same track. The $50 \mathrm{~Hz}$ field measured for this run is shown in figure 5-22. The pressure field magnitude for a $4 \mathrm{~km}$ aperture near the turnaround point is isolated and shown in figure 5-24. Segment 2 refers to the data taken along the NW leg where the source/receiver are opening in range, and segment 3 refers to the return leg where source/receiver are closing in range. Sound speed variability measured using the NRL T-string data for the two segments are shown in figure 5-23. For both segments, the location of the mixed layer in depth and the temporal variability was similar as determined by examining the mean and standard deviations of the temperature profiles. The modal interference pattern for the two tracks are very similar with most observable differences due to noise. Using a measure of the pressure on the hydrophone for a period of time when the $50 \mathrm{~Hz}$ source was turned off, SNR was determined to be about $20 \mathrm{~dB}$. Sliding window AR transforms with $1000 \mathrm{~m}$ apertures were applied to each of these data segments for estimating horizontal wavenumber content. For the estimated SNR and data aperture length, the theoretical variance of the estimates is very small at $6.28 \mathrm{e}-7 \mathrm{~m}^{-2}$ giving an error in the estimate of approxi- 

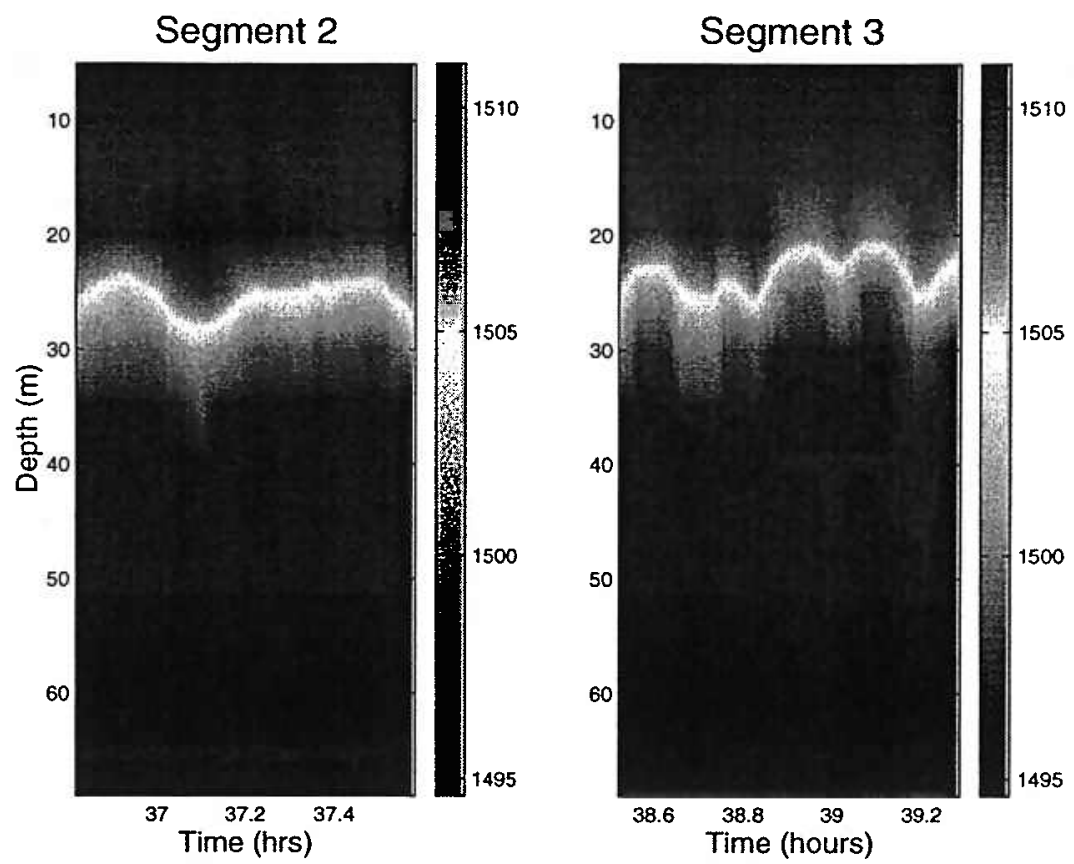

Figure 5-23: Sound speed variability measured on NRL T-strings during acoustic measurements along segments 2 and 3 . Colorbars indicate sound velocity in $\mathrm{m} / \mathrm{s}$.

mately $0.0003 \mathrm{~m}^{-1}$. The use of the theoretical variance is justified by the observation that the AR estimator performed as well or better then predicted on the time-series data in chapter 3. Figures 5-25-5-28 show the wavenumber evolution for the two different segments using two different representations.

Picking off the peak locations for the different mode numbers with range from the waterfall plots, modal evolution with range are shown in figure 5-29. Difference between maximum and minimum wavenumber estimates determined with range are an order of magnitude greater than the estimated measurement error. Thus, even given the presence of noise in the measured signals, the wavenumber estimates are shifted versions of one another for the overlapping source tracks. This is consistent with the wavenumber shift for a moving source given by Hawker [38] and also observed by Rajan et. al. [67]. From this observation, a method for correcting for the wavenumber shift is suggested along with a direct measure of modal group velocity from the data. In this analysis, the broadband effect of the moving source is removed by analyzing the data at a fixed frequency. The resulting wavenumber shift observed 


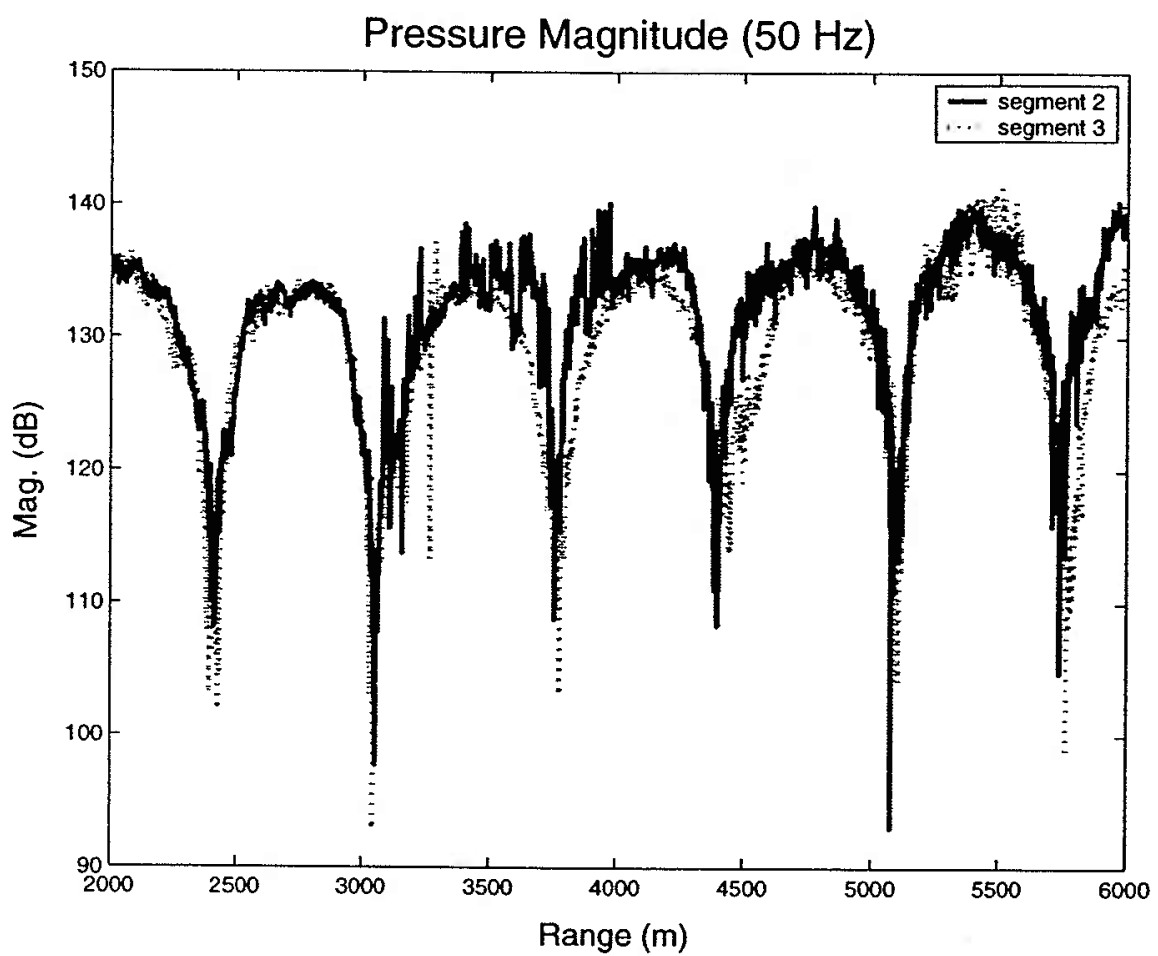

Figure 5-24: $50 \mathrm{~Hz}$ pressure magnitude data for overlapping tracks of segments 2 and 3

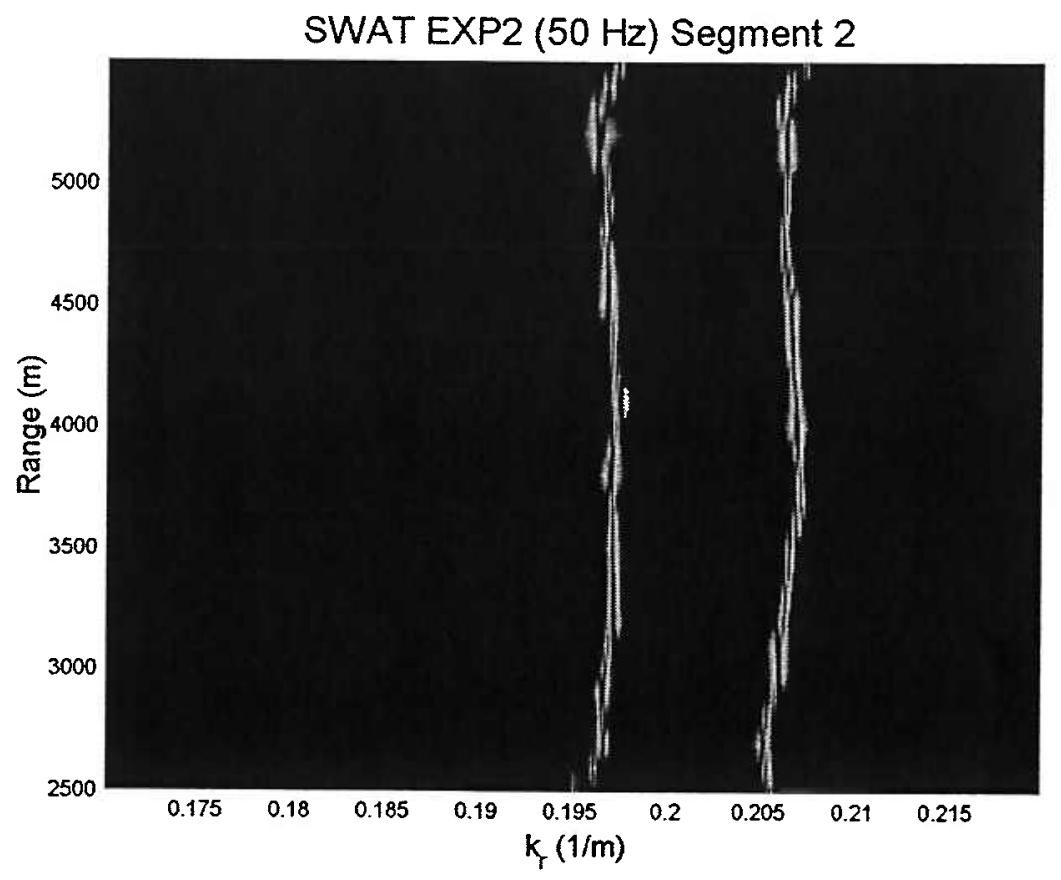

Figure 5-25: Modal evolution for $50 \mathrm{~Hz}$ data of segment 2. 


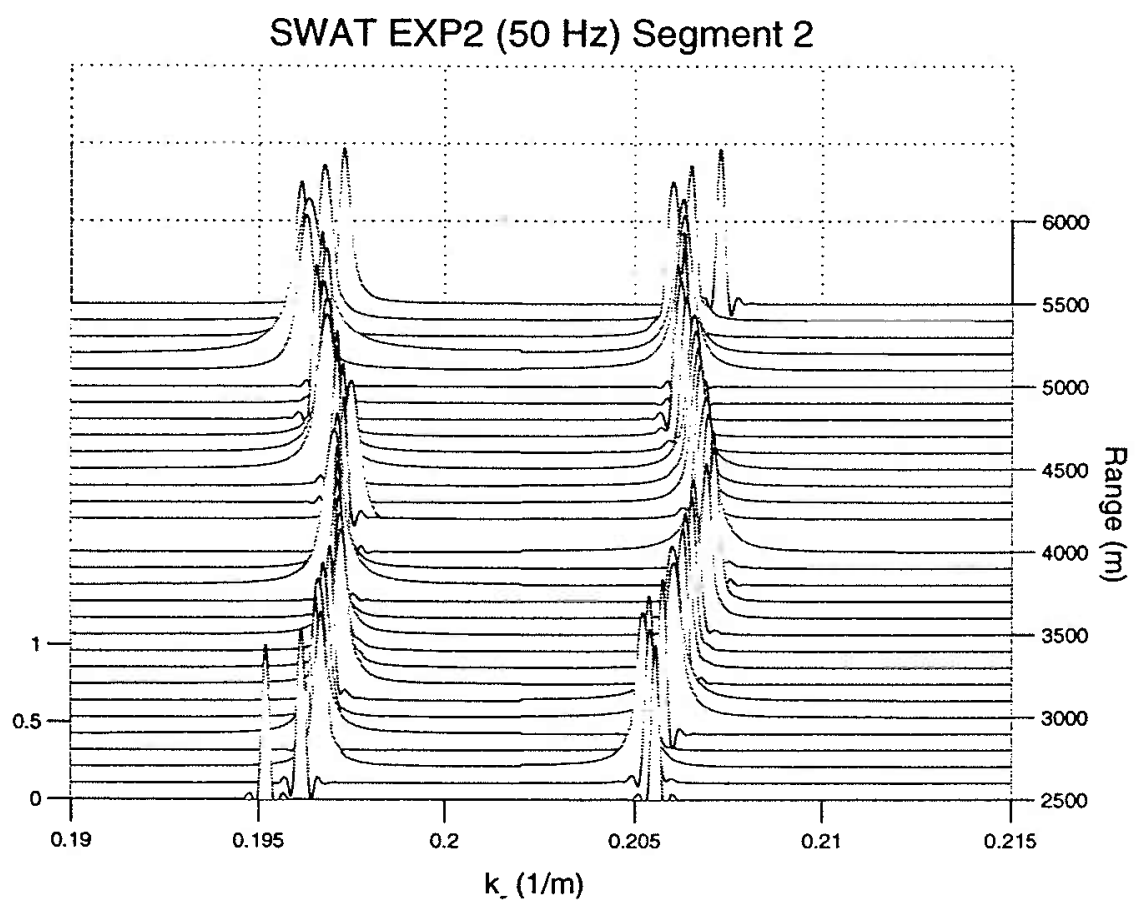

Figure 5-26: Waterfall plot of spectrum amplitudes with range for $50 \mathrm{~Hz}$ data of segment 2 .

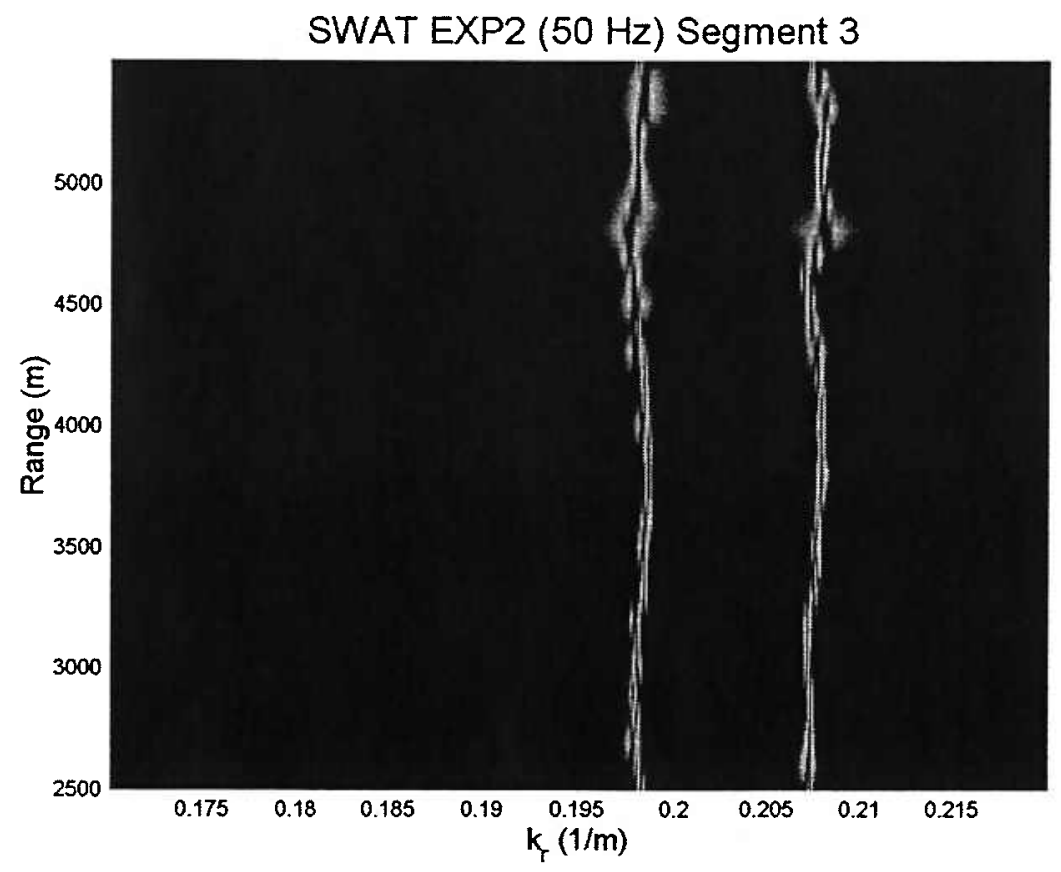

Figure 5-27: Modal evolution for $50 \mathrm{~Hz}$ data of segment 3. 


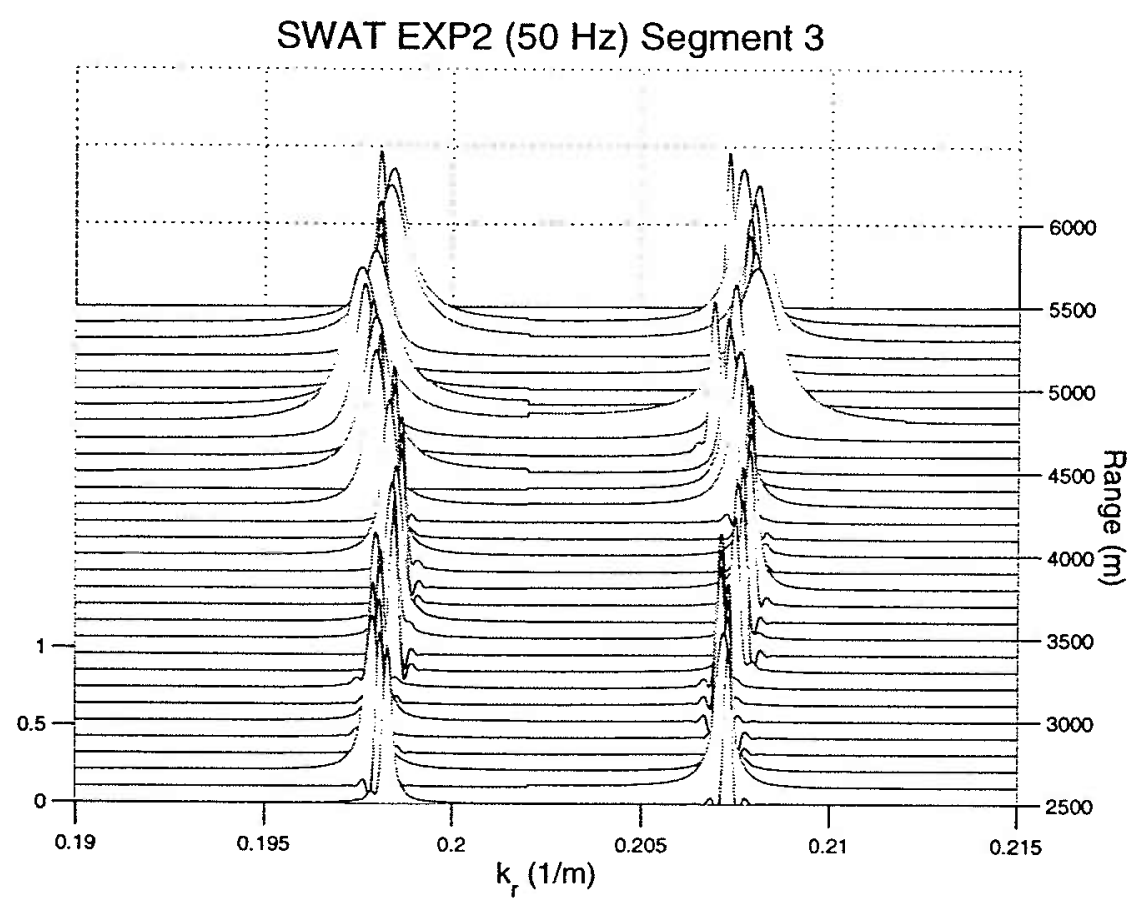

Figure 5-28: Waterfall plot of spectrum amplitudes with range for $50 \mathrm{~Hz}$ data of segment 3 .

in the data due to source motion, as predicted by both Hawker [38] and Schmidt and Kuperman [73] through the phase of the pressure field, must be accounted for in doing geoacoustic inversion.

As in the work by Rajan et. al. [67], the starting point for the analysis is equation (2.40) written as

$$
k_{n}^{*}=k_{n}\left(1 \pm \frac{v}{v_{n g}}\right)
$$

where $v$ is the source speed, positive when moving toward the receiver, and the source track is assumed to be along a line that goes through the receiver location. Estimates of group velocity were made from the differences obtained from the observed shift in wavenumbers determined from the data. For the individual wavenumbers determined along each track, the difference in wavenumbers can be expressed by,

$$
\Delta k_{n}^{*}=k_{n}^{*\left(v_{+}\right)}-k_{n}^{*\left(v_{-}\right)}=k_{n}\left(\frac{v_{+}}{v_{n}^{g}}-\frac{v_{-}}{v_{n}^{g}}\right)
$$

where $v_{ \pm}$are the relative velocities for the different tracks, and $k_{n}$ is the eigenvalue 


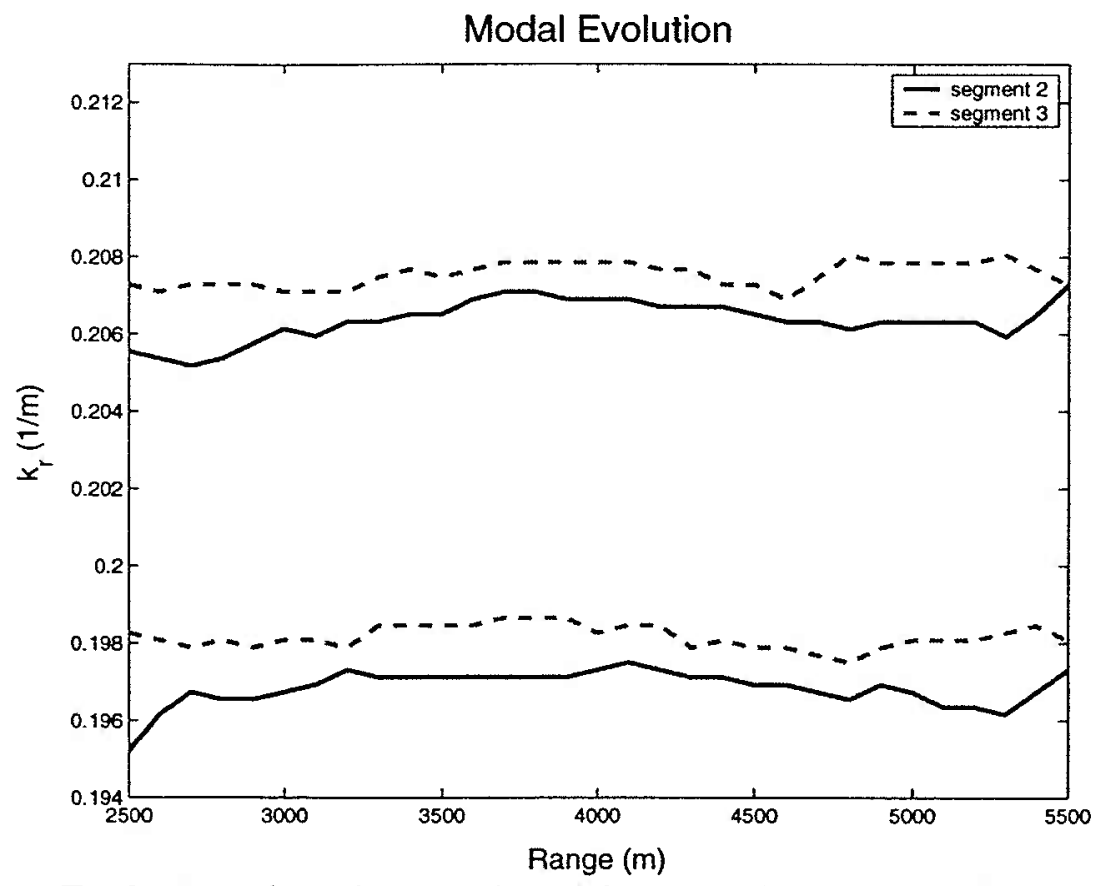

Figure 5-29: Evolution of modes 1 and 2 with range showing bias due to source motion over segments 2 and 3.

for a stationary source. For a source moving with a constant velocity back and forth along the same track, i.e. $v_{+}=-v_{-}$, the above expression can be simplified to give an expression for the group velocity, $v_{n}^{g}$, as,

$$
v_{n}^{g}=\frac{2 k_{n} v}{\Delta k_{n}^{*}}
$$

In the above, it remains to estimate $k_{n}$ from the shifted wavenumber. For a source moving out and back along the same path at a constant speed,

$$
k_{n}=\left(k_{n}^{*\left(v_{+}\right)}+k_{n}^{*\left(v_{-}\right)}\right) / 2,
$$

Combining (5.8) and (5.10), the group velocity can be written in terms of sums and differences of the shifted wavenumber.

$$
v_{n g}=\frac{\left(k_{n}^{*\left(v_{+}\right)}+k_{n}^{*\left(v_{-}\right)}\right)}{k_{n}^{*\left(v_{+}\right)}-k_{n}^{*\left(v_{-}\right)}} v
$$

Using the above expression, the modal group velocity can be calculated from the 
shifted wavenumber observations. The wavenumber estimates corrected using rangeaveraged group velocities for the apparent Doppler shift are shown in figure 5-30. The expected agreement between the two measurements is improved and an average value at a given range is suggested for use in inversion schemes. The calculated modal group velocities of $784.4 \mathrm{~m} / \mathrm{s}$ and $668.9 \mathrm{~m} / \mathrm{s}$ for the first two modes are about half the expected values when compared with the water sound speed. However, the source speed of $2 \mathrm{~m} / \mathrm{s}$ was very slow leading to small observed differences in the wavenumber estimates and a corresponding lack of resolution. Because the error estimate of the expected measure was small compared to the changes in individual wavenumbers with range, some range-dependence in the medium can be inferred. Thus, in estimating the error in the measurement of the modal group velocity, the error in the sum and difference measurements is used, where the expected wavenumber shift with range is assumed to be constant. The error in the sum of the wavenumbers with range is given by $\epsilon_{+}$and taken to be the standard deviation of the measured sum as a function of range divided by two. The error in the difference is given by $\epsilon_{-}$and taken to be the standard deviation of the measured wavenumber differences as a function of range. Using the above definition, the error in the group velocity measurement, $\epsilon_{v_{n}^{g}}$, can be expressed using standard techniques as a percentage of the expected value by [79]

$$
\frac{\epsilon_{v_{n}^{g}}^{g}}{v_{n}^{g}}=v \sqrt{\frac{\epsilon_{+}}{\left(k_{n}^{*\left(v_{+}\right)}+k_{n}^{*\left(v_{-}\right)}\right) / 2}+\frac{\epsilon_{-}}{\left(k_{n}^{*\left(v_{+}\right)}-k_{n}^{*\left(v_{-}\right)}\right)}}
$$

Using measured values in the above expressions, errors in the group velocity measurement due to wavenumber estimation errors were greater than $40 \%$. This can account for much of the error in the observed group velocity estimates. Other sources of error need to be investigated, including the effects due to displacement of the out and back tracks for this data.

Nevertheless, motivated by these experimental results, a numerical experiment was conducted using modal functions and eigenvalues determined using KRAKEN 


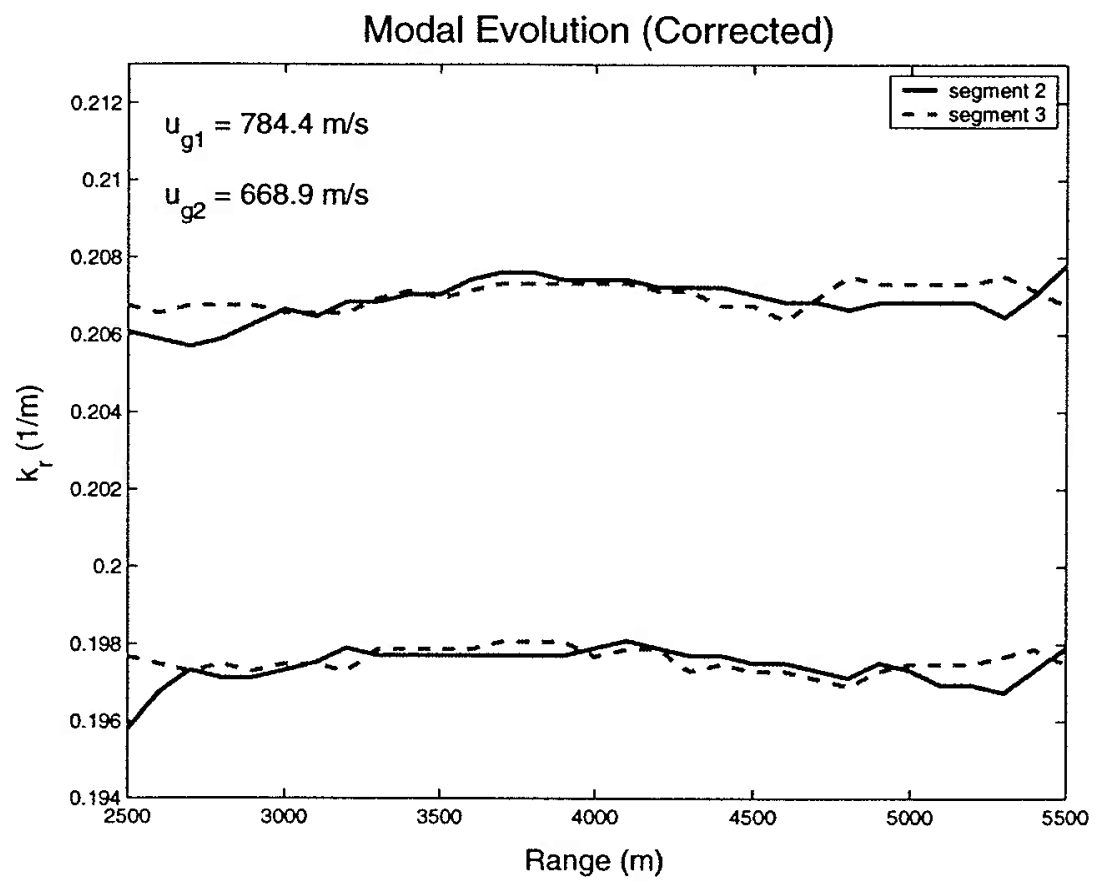

Figure 5-30: Doppler corrected wavenumber estimates for modes 1 and 2 over data segments 2 and 3.

\begin{tabular}{|c|c|c|}
\hline Depth $(\mathrm{m})$ & $c_{p}(\mathrm{~m} / \mathrm{s})$ & $\rho\left(\mathrm{g} / \mathrm{cm}^{3}\right)$ \\
\hline $0-75$ & 1510.0 & 1.0 \\
\hline $75+$ & 1600.0 & 1.6 \\
\hline
\end{tabular}

Table 5.1: Pekeris waveguide model parameters for moving source experiment.

for a Pekeris waveguide with properties given in table 5.1, using a source frequency of $50 \mathrm{~Hz}$. For the given input environment and source frequency, KRAKEN also outputs modal group velocities, which are given along with the mode numbers and phase velocities, for the Pekeris waveguide in table 5.2. Using the mode functions output from KRAKEN, complex pressure fields were generated as a function of range for a stationary source, and for sources moving with velocities of $\pm 10 \mathrm{~m} / \mathrm{s}$. The fields were generated for a $5 \mathrm{~km}$ aperture as a sum over the modes and corresponding 


\begin{tabular}{|c|c|c|c|}
\hline Mode No. & $k_{r}(1 / \mathrm{m})$ & $v_{p}(\mathrm{~m} / \mathrm{s})$ & $v_{g}(\mathrm{~m} / \mathrm{s})$ \\
\hline \hline 1 & 0.2055064180 & 1528.71 & 1499.07 \\
\hline 2 & 0.1974793421 & 1590.85 & 1476.45 \\
\hline
\end{tabular}

Table 5.2: Modal eigenvalues and amplitudes for $50 \mathrm{~Hz}$ Pekeris.

\begin{tabular}{|c|c|c|c|c|c|c|}
\hline Mode No. & $k_{i}^{\text {true }}(1 / \mathrm{m})$ & $k_{i}^{\text {est }}(1 / \mathrm{m})$ & $\%$ diff. & $v_{q}^{\text {true }}$ & $v_{g}^{\text {est }}$ & $\%$ diff \\
\hline \hline 1 & 0.2055064180 & 0.2055054887 & $4.5 \mathrm{e}-04$ & 1499.07 & 1498.95 & 0.008 \\
\hline 2 & 0.1974793421 & 0.1974895105 & $5.1 \mathrm{e}-03$ & 1476.44 & 1471.13 & 0.365 \\
\hline
\end{tabular}

Table 5.3: Wavenumber and group velocities estimated from moving source spectra.

wavenumbers using the simple expression,

$$
p(r)=\sum_{n=1}^{2} \frac{\Psi_{n s} \Psi_{n r}\left(k_{n}^{*}\right)}{\sqrt{k_{n}^{*} r}} e^{i k_{n}^{*} r}+w[r]
$$

where $\Phi_{n s}$ and $\Phi_{n r}$ are the mode amplitudes at the source and receiver, $k_{n}^{*}=k_{n}(1+$ $\frac{v}{v_{n g}}$ ) with $v_{n g}$ the modal group velocity, $v$ is the source speed, and $w[r]$ is Gaussian white noise. The complex fields with SNR of $40 \mathrm{~dB}$ generated for the different source motions are shown in figure 5-31.

Using the synthetic data, estimates were made of the wavenumber content for the different source velocities. Figure 5-32 shows the spectral estimates for the $10 \mathrm{~m} / \mathrm{s}$ moving source fields. The two estimates are shifted from one another with respect to the stationary value of the wavenumbers as indicated by the black lines. Using the full aperture and sampling the range every 5 meters, estimates of the shifted wavenumbers were obtained. Values of horizontal wavenumbers for the first two modes were then estimated as the average value of the estimates from the sources of opposing motion. The estimated wavenumbers compare very favorable with the true values for the given environment as given by KRAKEN. Group velocities were also determined which also compare well to the expected values as shown in table 5.3. The 

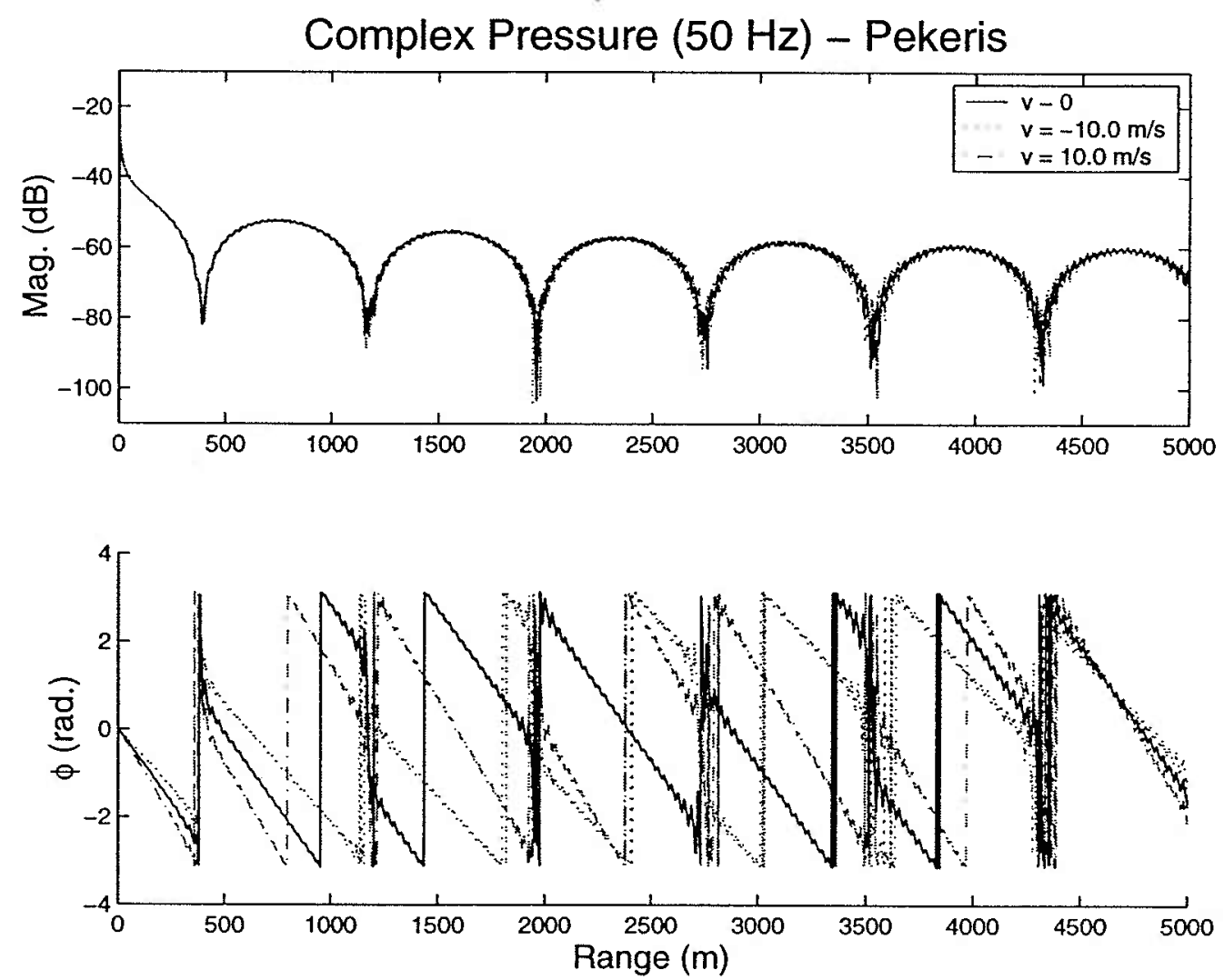

Figure 5-31: $50 \mathrm{~Hz}$ synthetic pressure fields generated for moving source analysis. $\mathrm{SNR}$ is $40 \mathrm{~dB}$. Source speeds were $10 \mathrm{~m} / \mathrm{s}$.

results from this experiment suggest that the wavenumber differencing technique used to estimate discrete wavenumbers for sources moving in opposing directions over the same environment is quite effective. Estimates of wavenumbers and group velocities agreed well with the true values. Relative to the MOMAX experiments, that had a typical source velocity of $2 \mathrm{~m} / \mathrm{s}$, the numerical results are shown for a high source speed in order to emphasize the observed wavenumber shift and reduce errors in the measurement of the wavenumber differences. However, the numerical experiment was also conducted for a source speed of $2 \mathrm{~m} / \mathrm{s}$ and yielded a small degradation in the measurement of the group velocity of the first mode which was estimated as $1478 \mathrm{~m} / \mathrm{s}$, or a $1.3 \%$ difference from the true value. These results indicate that the wavenumber differencing technique shows promise for measuring modal group velocity and merits further study both numerically and experimentally. In particular, 


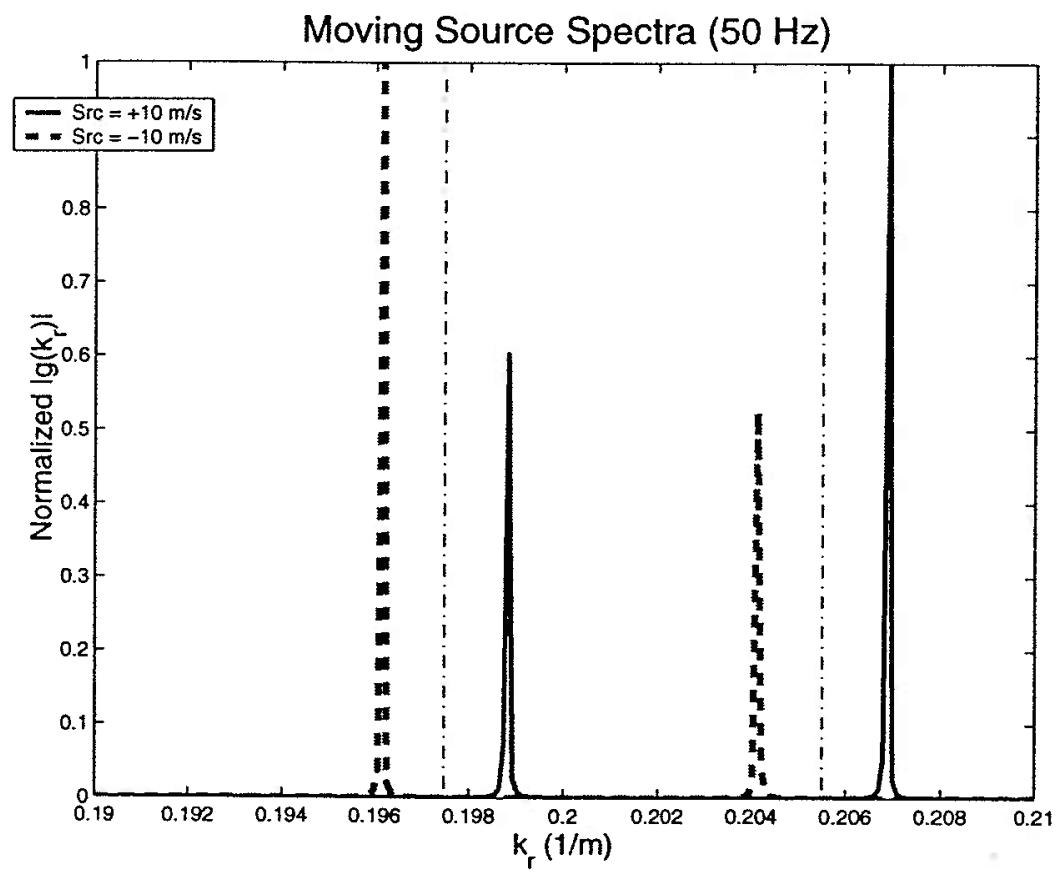

Figure 5-32: Spectral estimates for moving source data with stationary values of horizontal wavenumbers indicated by dashed line. Source speed was $10 \mathrm{~m} / \mathrm{s}$

for an experimental study, careful attention should be given to reducing errors in the wavenumber measurements. Much could be done in this regard by designing an experiment where the source is constrained to move out and back over exactly the same range-independent environment where the water column is well mixed and the sound speed is constant.

\subsection{Gelfand-Levitan Results}

Before leaving the analysis chapter, the Gelfand-Levitan method is considered in light of some of the practical aspects of making real ocean acoustic measurements. There are three issues in particular that affect how real measurements impact the application of the Gelfand-Levitan method. The first issue relates to the assumption of no allowable density discontinuity across the potential layer interfaces. This condition is violated in almost any realistic waveguide, where a density jump typically occurs at the water-bottom interface. It will be shown that this issue can be resolved through 
application of an impedance matching condition applied at the boundary. The second issue is related to the integration interval in wavenumber space of the point source spectrum to determine the Fourier transform of the reflection coefficient. As derived, the integral is taken over all wavenumber space. However, in an experiment, it is only possible to measure real propagation angles which limits the wavenumber integral to $\pm k_{o}$, where $\left|k_{o}\right|$ is the minimum water wavenumber. The truncated integration interval has an impact on the transform of the reflection coefficient similar to the problems associated with short aperture spectral analysis. The impact of truncating the integration interval on inversion using the Gelfand-Levitan approach will be discussed. A final study will address the issue of determining the plane-wave reflection coefficient from the shallow-water Green's function. It will be demonstrated that given an exact expression for the Green's function, the reflection coefficient can be extracted and sediment properties recovered.

\subsubsection{Effects of Density and Wavenumber Interval}

A numerical study was conducted to examine the effect of a density discontinuity at the water-bottom interface on inversion using the Gelfand-Levitan method. A deep water problem was considered with a sound velocity profile in the sediment consisting of a shallow minimum at 1 meter in depth, followed by a constant positive gradient with depth. The profile is described by,

$$
\begin{array}{ccc}
c(z) & = & 1540-(1540-1515) z, \quad 0 \leq z \leq 1 \\
c(z) & = & 1515+0.97 z, \quad 1<z \leq 145 \\
c(z)= & 1665, \quad z \geq 145
\end{array}
$$

and is shown in figure 5-35 as the exact profile. The density in the water column was assumed to be $1 \mathrm{~g} / \mathrm{cm}^{3}$ and in the sediment to be $1.5 \mathrm{~g} / \mathrm{cm}^{3}$. For the given environment, the reflection coefficient was calculated using the modified ThomsonHaskell propagator matrix approach of Mook [52]. 

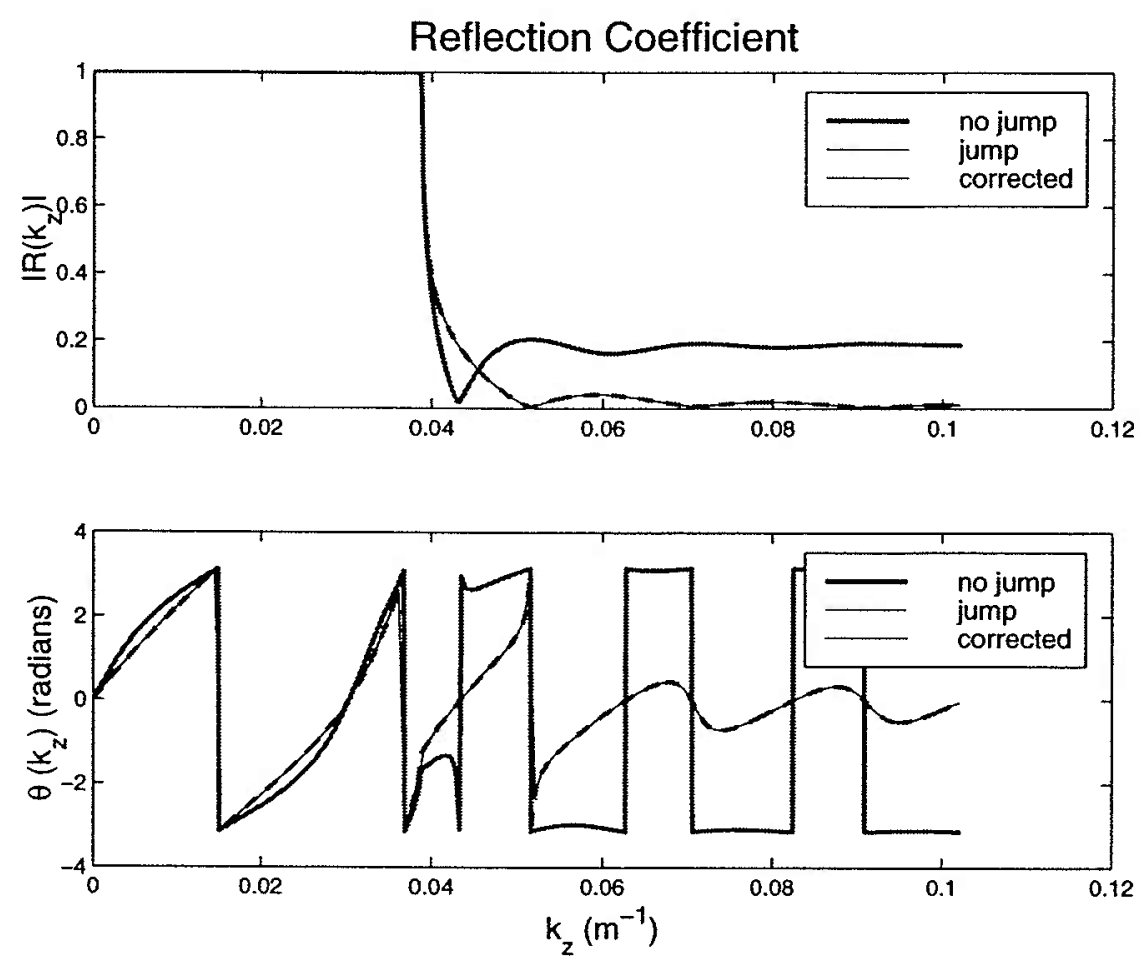

Figure 5-33: Reflection Coefficient at $20 \mathrm{~Hz}$ with and without density discontinuity at water-bottom interface.

To address the density discontinuity, an impedance matching condition was applied at the water-bottom interface. This condition is presented in Tolstoy and Clay [80] and gives a means for expressing the reflection coefficient just below the interface where the discontinuity occurs.

$$
R_{-}=\frac{\rho_{1} k_{z o}\left(1+R_{+}\right)-\rho_{o} k_{z 1}\left(1-R_{+}\right)}{\rho_{1} k_{z o}\left(1+R_{+}\right)+\rho_{o} k_{z 1}\left(1-R_{+}\right)}
$$

Here $R_{-}$is the reflection coefficient just below the interface, and $R_{+}$is the reflection coefficient just above the interface. The reflection coefficients determined using the Thomson-Haskell method both with and without the density jump are shown in figure 5-33. The effect of the density jump is evident in the magnitude plot where the reflection coefficient does not go to zero for large $k_{z}$. Using the impedance matching condition, the reflection coefficient determined just below the interface matches that for no discontinuity and approaches zero as required by the Gelfand-Levitan method. 
The Fourier transform of the reflection coefficient as a real function of depth and is plotted in figure 5-34 both with and without the effects of the density jump. The effect of the jump discontinuity is to impart an extra oscillation in the result at the upper depths that causes an offset from the continuous density case at the deeper depths. The Fourier transform results were used as input data to the GelfandLevitan equation to invert for the sound velocity in the sediment. Figure 5-35 shows the results for several cases. The input profile is shown in the background with the Merab [50] result plotted directly on top of it. This result is from the corrected reflection coefficient shown above where the wavenumber integral to determine the Fourier transform was taken out to 10 times the water wavenumber. The highly oscillating result shows the effect of not accounting for the density discontinuity. The final two results are for a reflection coefficient with no density jump and with the impedance matching condition applied where the wavenumber integral was truncated at $k_{o}$. This result shows the impact of truncating the wavenumber integral when taking the Fourier transform to have the greatest effect at large depths.

\subsubsection{Shallow-water Exact Inversion}

The final numerical experiment was simply a test to check that it was possible determine the reflection coefficient from the shallow-water Green's function using the relationship given by (2.14). It was thought that the poles in the Green's function might make the expression difficult to evaluate. This was tested using the shallowwater Green's function determined for the Pekeris waveguide illustrated in figure 5-36. This waveguide was chosen from an example given in Jensen et al. [41] with a frequency of $20 \mathrm{~Hz}$, source depth $=36 \mathrm{~m}$, receiver depth $=46 \mathrm{~m}$, and sound velocities given in the figure. The Green's function was determined from the exact expression for the reflection coefficient for a halfspace and compared to the results of OASES [72] and KRAKEN [59] as shown in figure 5-37. The agreement was good and the reflection coefficient was determined and plotted as a function of $k_{z}$ in fig- 


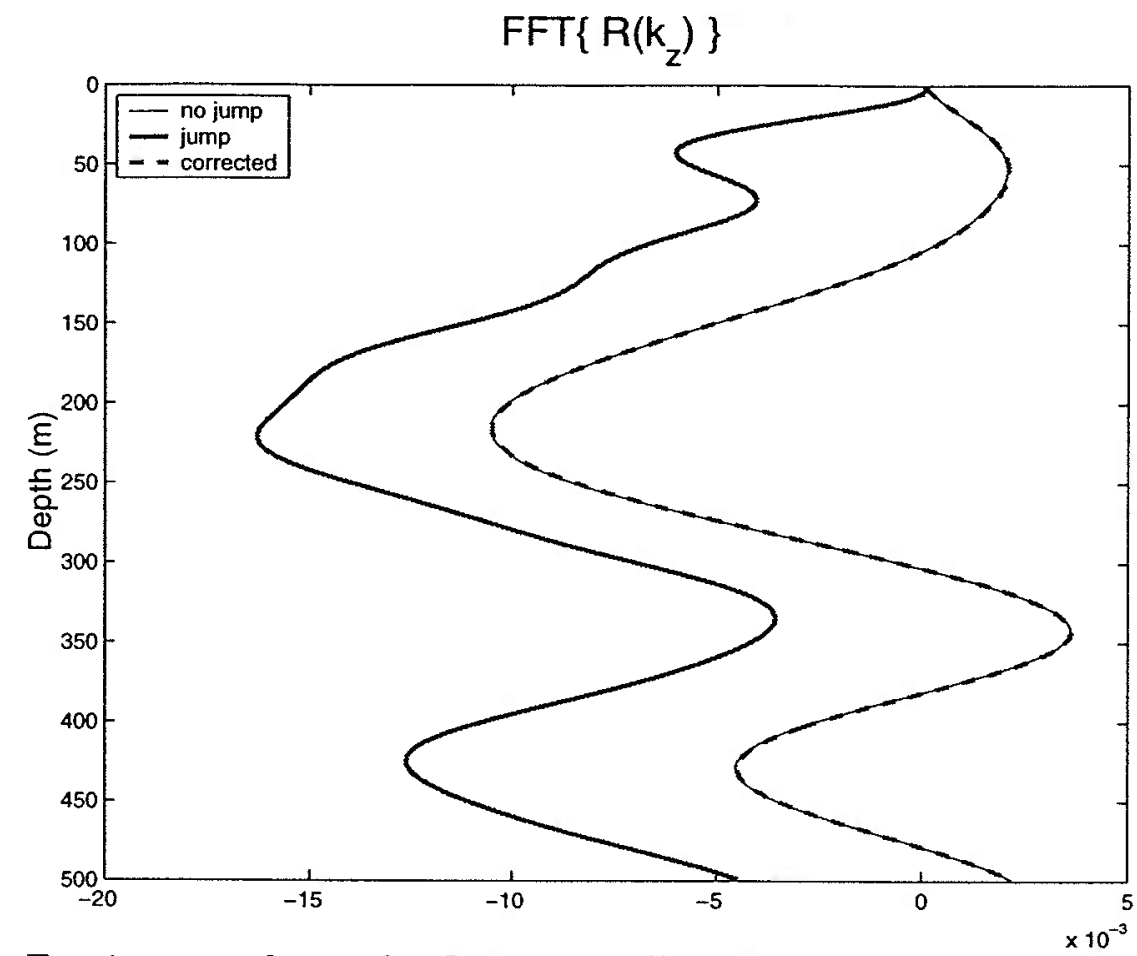

Figure 5-34: Fourier transform of reflection coefficient with and without density discontinuity.

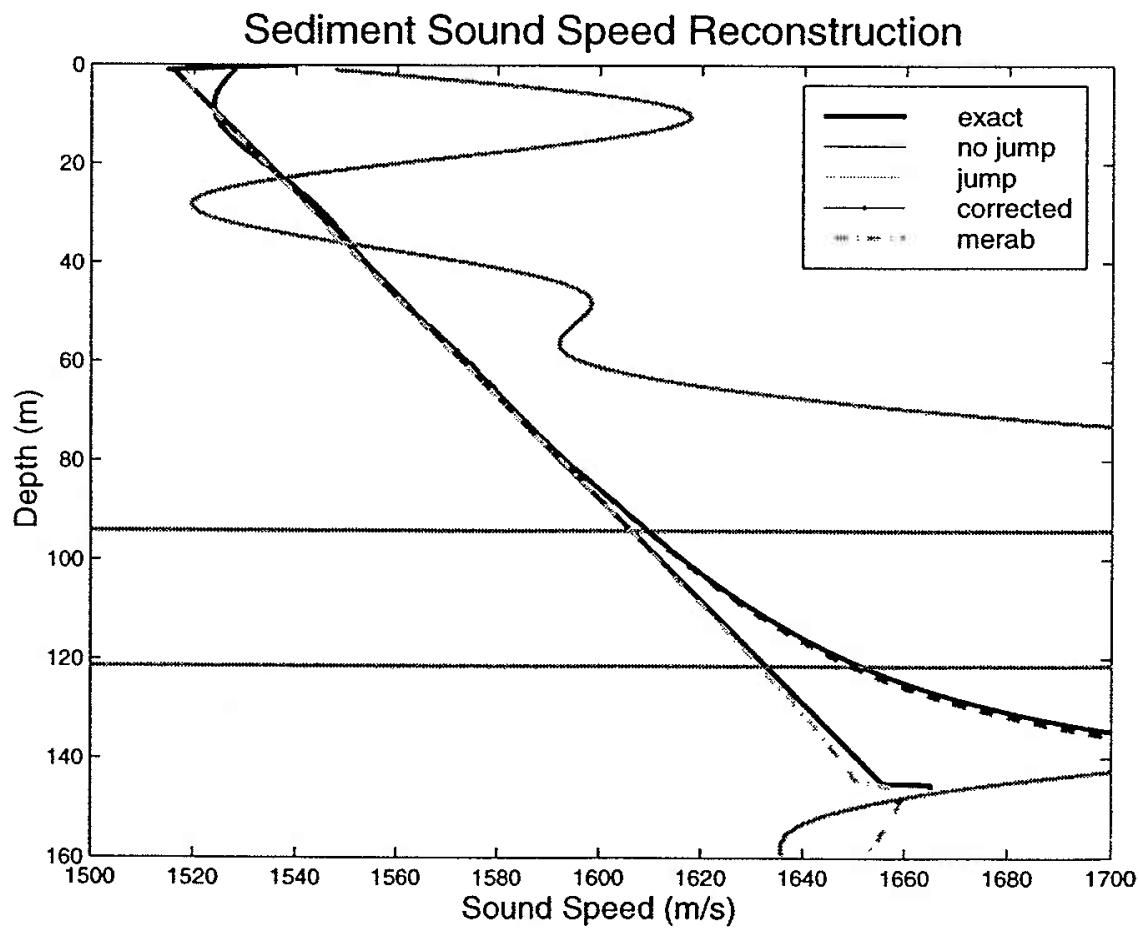

Figure 5-35: Comparison of inferred sound speed profile in sediment using the Gelfand-Levitan method. Results are shown with and without density discontinuity and for truncated wavenumber integrals. 


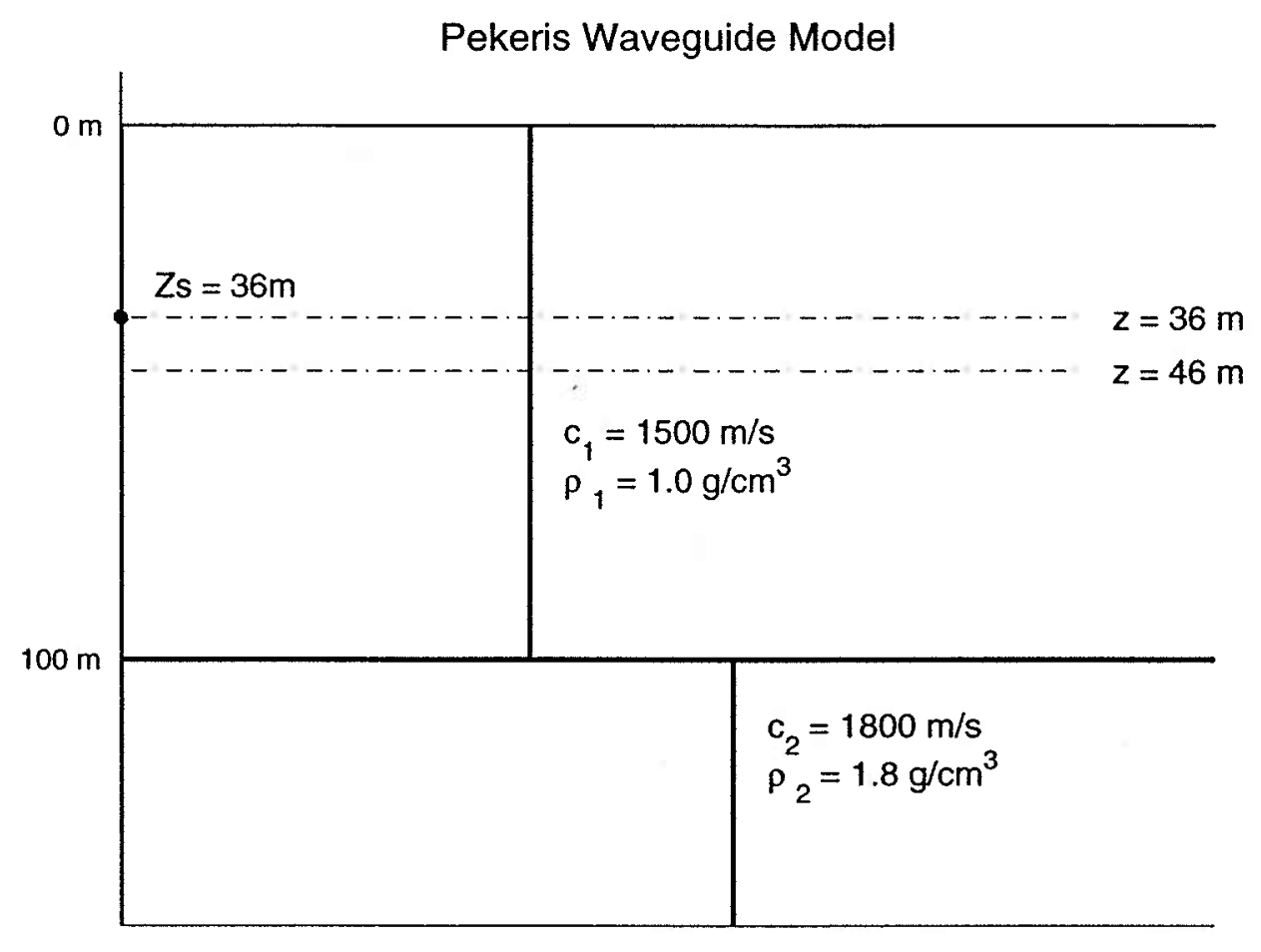

Figure 5-36: Pekeris environment used to calculate shallow water Green's function.

ure 5-38 where the density jump has been accounted for. The Fourier transform of the reflection coefficient and the resulting inversion are shown in figures 5-39 and 5-40. Good agreement is shown between the inversion and the input model. This demonstrates that a measure of the shallow-water Green's function might be used for determining the reflection coefficient for exact inversion. Work is in process for using these methods on Green's functions determined for real data in deep water [32]. The method has not been applied to shallow water data at this point. 


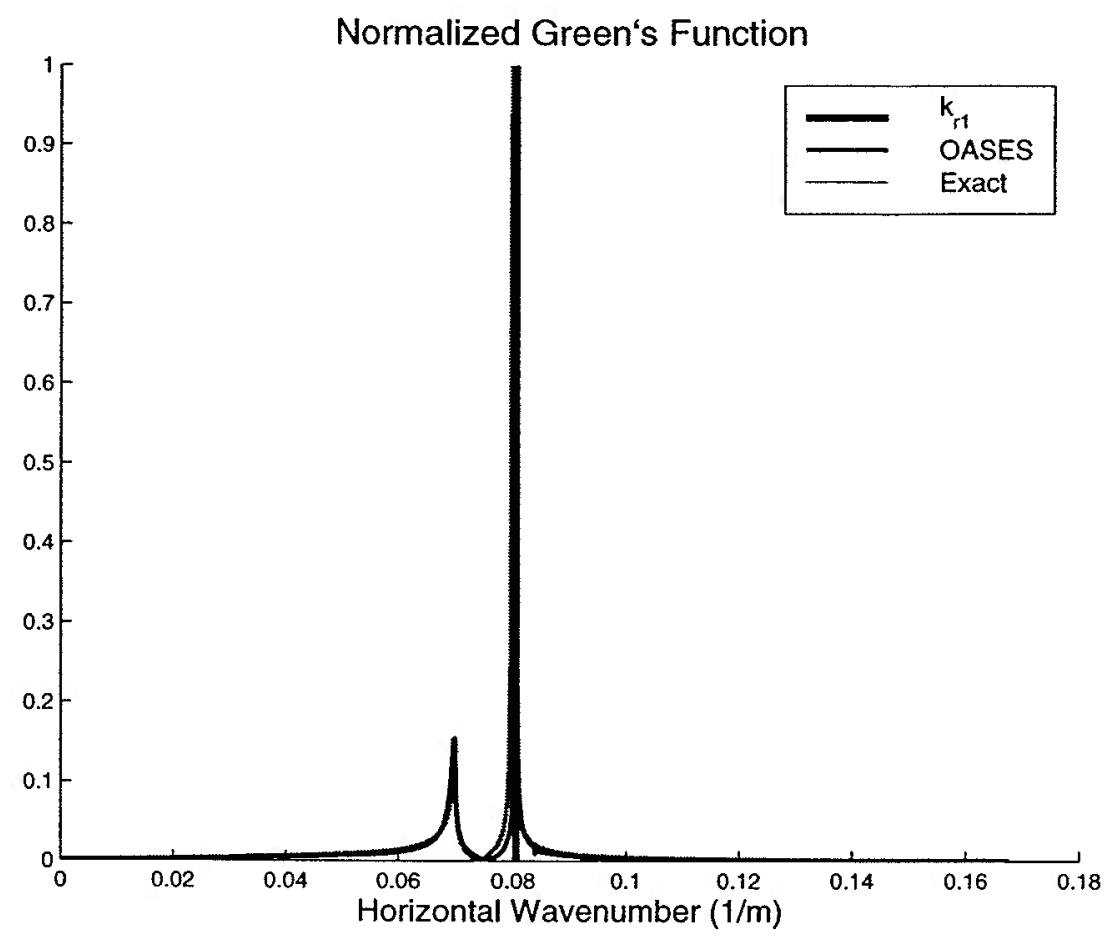

Figure 5-37: Exact Green's function for Pekeris problem compared to OASES and KRAKEN.

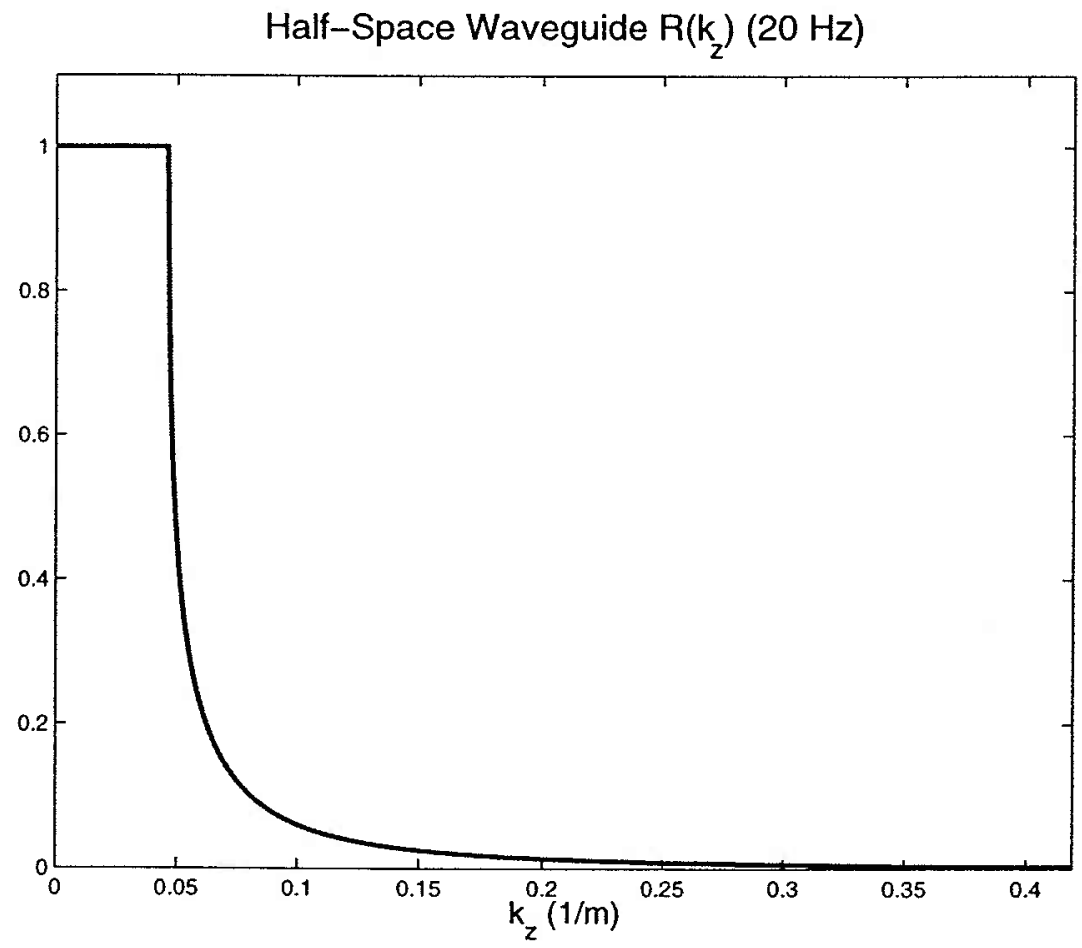

Figure 5-38: Reflection coefficient determined from shallow-water Green's function. 


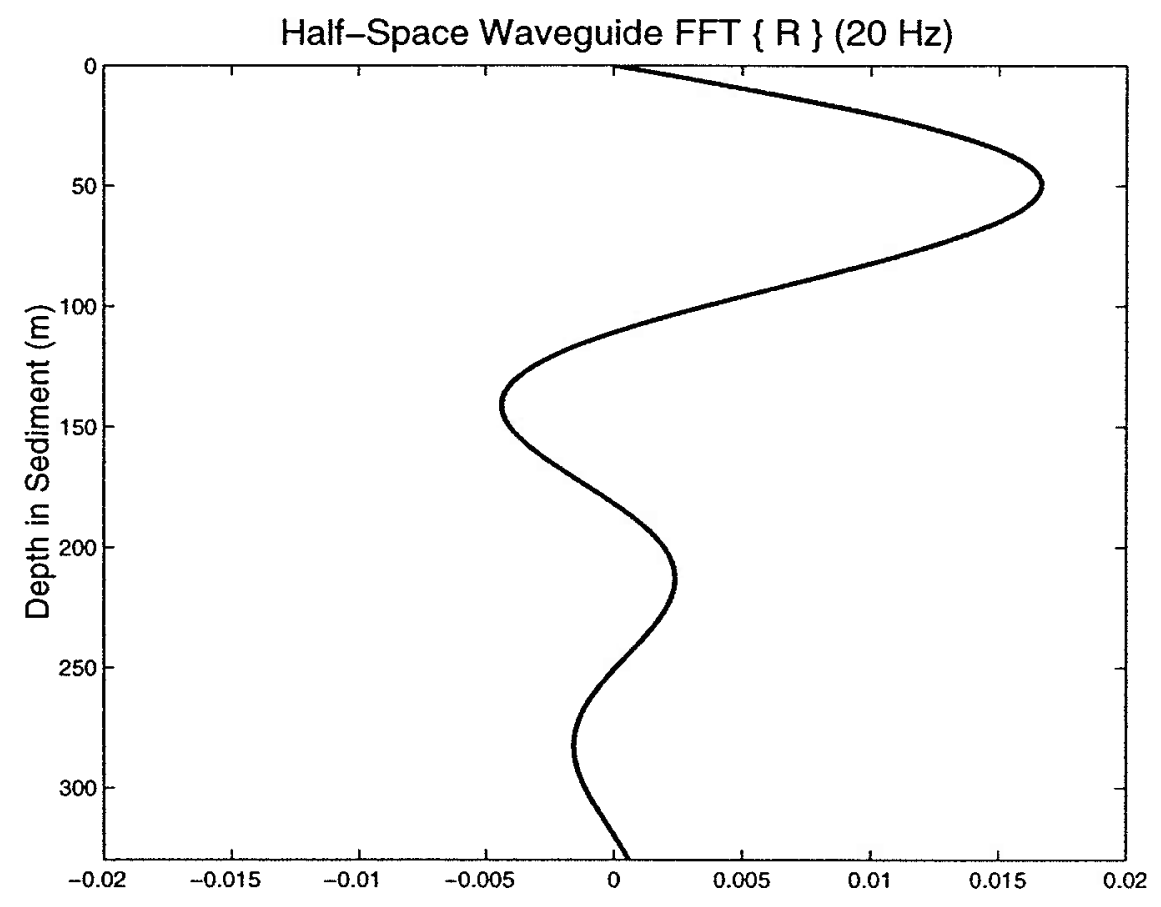

Figure 5-39: Fourier transform of Pekeris reflection coefficient with effect of density discontinuity removed.

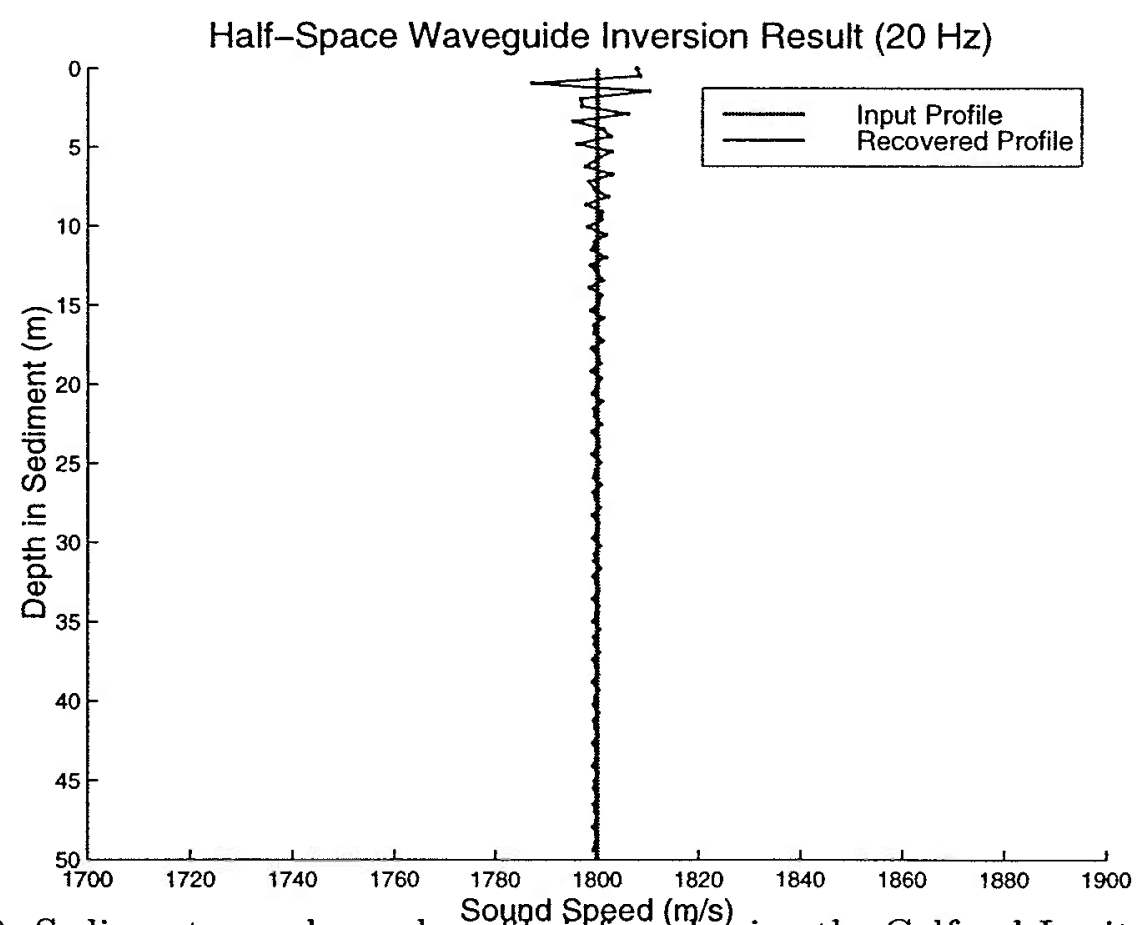

Figure 5-40: Sediment sound speed proflle inferred using the Gelfand-Levitan method for the Pekeris problem in figure 5-36. 


\section{Chapter 6}

\section{Summary and Conclusions}

In this thesis, the relationship between the spectrum of a point source acoustic field and the sediment properties of a shallow-water waveguide was described. Adiabatic mode theory was then used as a guide to illustrate how modes adapt to the local properties of the waveguide. This was demonstrated using synthetic data for a waveguide with properties that varied in three dimensions. The resulting modal maps could used as input data to infer local waveguide properties.

As part of the ONR/SPAWAR inversion techniques workshop, synthetic data were provided for analysis. For TC3, the seafloor was flat and the sediment properties varied with range. It was demonstrated that detection of range-variability in the sediment using wavenumber analysis required high-resolution methods to fully resolve closely spaced wavenumbers in the local spectra. A high-resolution method based on autoregressive spectral estimation was described and characterized for identifying local modal content using short-aperture data. The high-resolution estimator was then used to examine wavenumber content for a waveguide including sound speed perturbations in the water column due to internal waves.

An experimental method for measuring three-dimensional acoustic fields was described for use in extracting spatially-varying wavenumber content. The resulting measurements provided data on synthetic aperture horizontal arrays of arbitrary 
shape determined by the source and receiver motions. The full 2-D horizontal arrays were sparsely populated requiring that data be mapped to a range grid and analyzed using methods based on the zeroth-order Hankel transform. Modal eigenvalues were extracted from select pieces of data and used as input data for perturbative inversion. It was shown that interpretation of the wavenumber spectra must take into account source/receiver motions. In particular, a Doppler shift in the spectrum was observed for an experiment where the source was moving with a constant velocity.

In addition to methods and analysis based on perturbative inversion methods, the Gelfand-Levitan method was discussed as a means for geoacoustic inversion. The relationship between the point-source spectrum and the plane-wave reflection coefficient of the water-bottom sediment was discussed. It was shown that the full spectrum, including the discrete values, could be used to determine the reflection coefficient for the shallow-water case. A simple method was demonstrated to account for the density jump at the water-bottom interface. Finally, the effect of using wavenumber content corresponding to only real angles of propagation was examined. This work suggests the potential use of MOMAX type data for doing exact geoacoustic inversion.

\subsection{Conclusions}

Much of this thesis work was focused on a means to provide data for doing perturbative geoacoustic inversion for spatially varying waveguides. It was demonstrated that a tool was necessary for extracting the discrete wavenumber spectrum for shortaperture data. An autoregressive spectral estimator was examined for use as a wavenumber estimator. It was demonstrated that the estimator performed well relative to other high-resolution estimators for determining the frequency content of a signal with noise. The same analysis was applied to determine wavenumber content for a synthetic acoustic field having known eigenvalues with similar results. An advantage over the other methods, based on eigen-decomposition, is that no a priori 
information regarding the signal being analyzed is required, such as the number of propagating modes. For the range-dependent inversion workshop TC3, it was shown that a reduction in aperture of approximately two could be used to resolve and extract modal content. It was shown that the location and shape of an intrusion in the sediment could be determined exactly. This result is particularly noteworthy in that for the inversion workshop, it was the only method capable of determining the nature of the intrusion in the sediment.

In the study of internal wave effects on wavenumber estimates, it was observed that the sound speed perturbations in the water column do not negatively impact the detection of propagating modes. Although mode coupling is observed when compared to the unperturbed sound speed, the effect is to transfer energy to modes which are consistent with the boundary conditions of the waveguide. The net effect appears to be that mode coupling yields more information which might be used in the perturbative inverse algorithm, i.e., modes that may not otherwise be excited are now identified.

For the MOMAX experiments described, it was shown that the synthetic apertures created by the drifting GPS navigated buoys were of very high quality. Relative source and receiver ranges and range rates could be determined directly from the phase measurements. For the single-mode experiment, agreement was obtained between the AR estimator and the phase derivative for examining the range evolution of horizontal wavenumber for the propagating mode. For a case where the aperture opened along a radial, wavenumber estimates were used as input data for perturbative inversion. The resulting sediment sound velocity profile was consistent with a sandy sediment as expected for the region. Wavenumber-based inversion was also performed for a moving source/receiver configuration. The field determined from the profile resulting from the extracted wavenumbers matched the data quite well. Although the moving source/receiver configuration made it difficult to assign the inversion results to a specific geographic location, the results suggest that wavenumber analysis techniques 
as a means for generating transmission loss curves for rapid assessment in situations where source and receiver positions are in constant motion. Related to this result was the observation that wavenumber estimates where shifted for an experiment where the source traversed the same local environment along an outgoing and incoming track. It was shown that a better estimate of the true wavenumbers could be obtained by correcting for the Doppler shift in the spectrum due to the source motion. From this analysis, a method was suggested from which modal group velocity could be estimated from the observed Doppler shift for a moving source. The results of a numerical study based on wavenumber estimates for a modal field with shifted eigenvalues verified these results and suggests further experimental work.

Finally, the numerical studies using the Gelfand-Levitan method suggest potential use of MOMAX type data for performing exact geoacoustic inversions. It was shown that for wavenumbers corresponding to real angles of propagation, inversion results could be obtained to a depth of one or two wavelengths in the sediment. It was shown that the density jump at the water-bottom interface could be accommodated by requiring a single point measurement of the density at the interface. It was also shown numerically that the shallow-water, depth-dependent Green's function could be used to determine the plane-wave reflection coefficient at the bottom for use in the Gelfand-Levitan equation.

\subsection{Suggestions for Future Work}

The autoregressive wavenumber estimator used for this work provides reliable wavenumber estimates only for regions of local waveguide invariability. In the case of a constant bathymetric slope where the sediment properties follow the bathymetry, the requirement of local invariability is a major deficiency. One way wavenumber estimates might be improved in this case is through the implementation of a time-varying autoregressive estimator (TVAR). The TVAR estimator allows for the coefficients of 
the parametric signal model to be time-varying, or in this application, range-varying. The effects of internal waves on wavenumber estimates is another area that deserves further work. The limits of wavenumber estimation in the presence of stronger and stronger internal wave fields should be studied.

A related area for research is the effect on wavenumber estimates due to the shape of intrusions in the sediment for a waveguide with constant bathymetry. For the inversion workshop, a rectangular-shaped intrusion was identified. An interesting study would be the identification of other regular shaped intrusions such as a semicircle or triangle. This might complement work relating to the recent GeoClutter [56] experiments, where discrete features in the sediment are studied as contributors to both propagating and scattered acoustic fields. This work might also provide a means to determine range dependent features of the environment which could be used along with the global search methods for doing geoacoustic inversion.

From the experimental work, it was shown that the measured spectra are influenced by the source and receiver motions. A method was described for inferring the group velocity from the shifted wavenumber spectra. An experiment designed to specifically measure shifted spectra for group velocity measurements is suggested where the source is towed at a high rate of speed to maximize the Doppler shift and increase the observed wavenumber differences for increased fidelity in the difference measurements. A similar experiment might also be designed to provide data along a radial at constant bearing for a long synthetic aperture to provide data for estimating the plane-wave reflection coefficient from the shallow-water Green's function. This would require additional work in the estimation of the Green's function from data. In this thesis work, the far-field approximation to the Hankel function was used, where an exact Hankel transform should be employed for the best estimate of the total spectrum. 


\section{References}

[1] H. Akaike, Power Spectrum Estimation through Autoregressive Model Fitting. Ann. Inst. Stat. Math., 21, pp 407-419, (1969).

[2] H. Akaike, A New Look at the Statistical Model Identification, IEEE Trans. Autom. Control, AC-19, pp. 716-723, (1974).

[3] J. R. Apel, M. Badiey, C-S Chiu, S. Finette, R. Headrick, J. Kemp, J. F. Lynch, A. Newhall, M. H. Orr, B. H. Pasewark, D. Tielbuerger, A. Turgut, K .v. d. Heydt, and S. Wolf, An overview of the 1995 SWARM shallow-water internal wave acoustic scattering experiment. IEEE J. Ocean. Eng., 22, (1997).

[4] A. B. Baggeroer, Confidence Intervals for Regression (MEM) Spectral Estimates, IEEE Trans. Inform. Theory, IT-22(5), pp. 534-545, (1976).

[5] A. B. Baggeroer, Sonar Signal Processing. in Applications of Digital Signal Processing, ed. A. V. Oppenheim. (Prentice-Hall, Englewood Cliffs, NJ, 1978).

[6] R. Beals, P. Deift, and C. Tomei, Direct and inverse scattering on the line. (American Math. Soc., Providence, RI, 1988).

[7] K. M. Becker, G. V. Frisk, and L. N. Connor, Three-dimensional modal mapping in shallow water using simulated acoustic field data. J. Acoust. Soc. Am., 101(5), 3160 (1997). 
[8] K. M. Becker, G. V. Frisk, and L. N. Connor, Modal evolution in a threedimensionally varying shallow water waveguide. J. Acoust. Soc. Am., 102(5), 3143 (1997).

[9] K. M. Becker and G. V. Frisk, Wavenumber extraction techniques for a threedimensionally varying shallow water waveguide: a comparison. Proceeding of the 16th International Congress on Acoustics and the 135th Meeting of the Acoustical Society of America, Seattle, WA, 20-26 June 1998, pp. 2433-2434.

[10] K. M. Becker and G. V. Frisk, High resolution modal mapping in a complex shallow-water environment. J. Acoust. Soc. Am., 105(2), 1310 (1999).

[11] G. H. Brooke, D. J. Thomson, and G. R. Ebbeson, PECan: A Canadian Parabolic Equation Model for Underwater Sound Propagation. J. Comp. Acoustics, 9(1), (2001).

[12] K. Chadan and P. C. Sabatier, Inverse Problems in Quantum Scattering Theory. (Springer-Verlag, New York, NY 1977).

[13] N. R. Chapman, S. A. Chin Bing, and D. King, ONR/SPAWAR Inversion Techniques Workshop Report. (in process September 2001).

[14] S. A. Chin-Bing, D. B. King, and J. D. Boyd, The Effects of Ocean Variability on Underwater Acoustic Propagation Forecasting, in Oceanography and Acoustics: Prediction and Propagation Models, ed. A. R. Robinson and D. Lee, (AIP Press, New York, 1994).

[15] M. D. Collins, W. A. Kuperman, and H. Schmidt, Nonlinear inversion for oceanbottom properties. J. Acoust. Soc. Am., 92(5), 2770-2783 (1992).

[16] T. A. Davies, J A. Austin, Jr., M. B. Lagoe, and J. D. Milliman, Late Quaternary sedimentation off New Jersey: New results using 3-D seismic profiles and cores. Marine Geology, 108, (1992). 
[17] P. Deift and E. Trubowitz, Inverse Scattering on the Line. Comm. Pure Appl. Math., vol. XXXII, 121-251 (1979).

[18] S. E. Dosso, J. M. Ozard, and J. A. Fawcett, Inversion of acoustic field data for bathymetry and bottom sound speed via simulated annealing. in Acoustic Signal Processing for Ocean Exploration, edited by J. M. F. Moura and I. M. G. Lourtie (Kluwer Academic, Dordrecht, 1993), pp. 51-56.

[19] J. Doutt, G. V. Frisk, and H. Martell, Determination of Distance Between a Moving Ship and Drifting Buoys to Centimeter-Level Accuracy at Sea Using L1 Phase GPS Receivers and Differential Moving-Base Kinematic Processing. Proceedings of the 11th International Technical Meeting of the Satellite Division of the Institute of Navigation., 1301 (1998).

[20] J. A. Doutt, G. V. Frisk, and H. Martell, Using GPS at Sea to Determine the Range Between a Moving Source Ship and a Drifting Buoy to Centimeter-Level Accuracy. Oceans '98 Conference Proceedings, Nice France, (28 September-1 October 1998).

[21] G. R. Ebbeson, D. J Thomson, and G. H. Brooke PE solutions to some internalwave benchmark problems. J. Acoust. Soc. Am., 109 (5 pt. 2), 2333 (2001).

[22] G. R. Ebbeson, personal communication (July 2001).

[23] M. R. Fallat and S. E. Dosso, Geoacoustic inversion via local, global, and hybrid algorithms. J. Acoust. Soc. Am., 105(6), 3219-3230 (1999).

[24] H. Fernandez, Migration of Range Dependent Shallow Water Acoustic Fields. Summer Student Fellow Report, WHOI, (1985).

[25] G. V. Frisk, A. V. Oppenheim, and D. R. Martinez, A Technique for measuring the plane-wave reflection coefficient of the ocean bottom. J. Acoust. Soc. Am., 68(2), 602-612 (1980). 
[26] G. V. Frisk, J. F. Lynch, and S. D. Rajan, Determination of compressional wave speed profiles using modal inverse techniques in a range-dependent environment in Nantucket Sound. J. Acoust. Soc. Am., 86(5), 1928-1939 (1989).

[27] G. V. Frisk, Inverse Methods in Ocean Bottom Acoustics. in Oceanographic and Geophysical Tomography, edited by Y. Desaubies, A. Tarantola, and J. ZinnJustin, (Elsevier Science Publishing Co., New York, 1990).

[28] G. V. Frisk, Ocean and Seabed Acoustics: A Theory of Wave Propagation. (P T R Prentice Hall, Englewood Cliffs, New Jersey, 1994).

[29] G. V. Frisk, K. M. Becker, L. N. Connor, J. A. Doutt, and C. J. Sellers, Highresolution three-dimensional measurements of low-frequency sound propagation in shallow water. J. Acoust. Soc. Am., 103(5), (1998).

[30] G. V. Frisk and K. M. Becker, Modal Evolution and Inversion in Laterally Varying Shallow-Water Waveguides. Proceedings of the International Conference on Acoustics, Noise, and Vibration, Montreal, Quebec, Canada, (August 2000).

[31] G. V. Frisk, K. M. Becker, and J. A. Doutt, Modal Mapping in Shallow Water Using Synthetic Aperture Horizontal Arrays, Proceedings Oceans 2000 MTS/IEEE Conference and Exhibition, Providence, RI, (September 2000).

[32] G. V. Frisk, L. L. Souza, and K. M. Becker, Application of the Gelfand-Levitan Method to Inverse Problems in Seabed Acoustics. Proceeding of the 2001 SIAM Annual Meeting, San Diego, CA, (9-13 July 2001).

[33] G. V. Frisk, The Relationship Between Low-Frequency Phase Rate and SourceReceiver Motion in Shallow Water: Theory and Experiment. Proceedings of the 17th International Congress on Acoustics, Rome, Italy, (2-7 September 2001). 
[34] I. M. Gelfand and B. M. Levitan, On the Determination of a Differential Equation from its Spectral Function. Amer. Math. Soc. Trans., Series 2, vol. 1, 253-304, (1955).

[35] P. Gerstoft, Inversion of seismoacoustic data using genetic algorithms and $a$ posteriori probability distributions. J. Acoust. Soc. Am., 95(2), 770-782 (1994).

[36] E. L. Hamilton, Geoacoustic modeling of the sea floor. J. Acoust. Soc. Am., 68(5), 1313-1340 (1980).

[37] C. H. Harrison and M. Siderius, Correlations between search parameters in geoacoustic inversion. in Proceedings of the Fifth European Conference on Underwater Acoustics, ECUA 2000, edited by P. Chevret and M. E. Zakhara. (Lyon, France 2000).

[38] K. E. Hawker, A normal mode theory of acoustic Doppler effects in the oceanic waveguide. J. Acoust. Soc. Am., 65(3), (1979).

[39] J. P. Hermand and P. Gerstoft, Inversion of broadband multitone acoustic data from the Yellow shark summer experiments. IEEE J. Oceanic Eng., 21, 324-346 (1996):

[40] ONR/SPAWAR Inversion Techniques Workshop, (15-18 May 2001). http://itworkshop.nrlssc.navy.mil/

[41] F. B. Jensen, W. A. Kuperman, M. B. Porter, and H. Schmidt, Computational Ocean Acoustics. (AIP Press, New York, 1994).

[42] S. M. Kay and S. L. Marple Jr., Spectrum Analysis - A modern Perspective, Proceedings of the IEEE, 69(11), (1981).

[43] G. Kitagawa and W. Gersch, Smoothness Priors Analysis of Time Series. (Springer-Verlag, New York, 1996). 
[44] J. A. Kong, Electromagnetic Wave Theory, 2nd edition. (John Wiley and Sons, New York, 1990).

[45] W. A. Kuperman, M. D. Collins, and H. Schmidt, A fast simulated annealing algorithm for inversion of marine sediment seismo-acoustic parameters. in Shear Waves for Marine Sediments, edited by J. M. Hovem, M. D. Richardson, and R. D. Stoll (Kluwer Academic, Dordrecht, 1991), pp. 521-528.

[46] R. M. Lewitt, Reconstruction Algorithms: Transform Methods. Proceedings of the IEEE, 71(3), (1983).

[47] LWAD 99-1 Participants, Littoral Warfare Advanced Development (LWAD 99-1) Quicklook Briefing Proceedings (27 April 1999).

[48] J. F. Lynch, S. D. Rajan, and G. V. Frisk, A comparison of broadband and narrow-band modal inversions for bottom geoacoustic properties at a site near Corpus Christi, Texas. J. Acoust. Soc. Am., 89(2), 648-665 (1991).

[49] S. L. Marple Jr., Digital Spectral Analysis with Applications. (Prentice-Hall, Inc., Englewood Cliffs, NJ 1987).

[50] A. A. Merab, Exact Reconstruction of Ocean Bottom Velocity Profiles from Monochromatic scattering Data . (Ph.D. dissertation, MIT/WHOI, WHOI-88$12,1988)$.

[51] J. R. McLaughlin and S. Wang, Recovery of a vertically stratified seabed in shallow water from acoustic data. (Preprint, Department of Math. Sciences, Rensselaer Polytechnic Institute, Troy, New York, 1998).

[52] D. R. Mook, The numerical synthesis and inversion of acoustic fields using the Hankel transform with application to the estimation of the plane wave reflection coefficient of the ocean bottom. (Sc.D. dissertation, MIT E.E. , 1983). 
[53] C. A. Nittrouer and J. H. Kravitz, STRATAFORM: A program to study the creation and interpretation of sedimentary strata on continental margins. Oceanography, 9, (1999).

[54] K. Ohta, Analysis of Modal Evolution Caused by Weakly a Range-Dependent Seabed in Shallow Water and It's Application to Inversion for Geoacoustic Properties . (Ph.D. dissertation, MIT/WHOI, WHOI-93-35, 1993).

[55] K. Ohta and G. V. Frisk, Modal Evolution and Inversion for Seabed Geoacoustic Properties in Weakly Range-Dependent Shallow-Water Waveguides. IEEE J. Oceanic Engineering, 22, 501-521 (1997).

[56] Office of Naval Research, Geologic Clutter Initiative. http ://www.onr.navy.mil/sci_tech/ocean/geoclutter.htm

[57] A. D. Pierce, Extension of the method of normal modes to sound propagation in and almost-stratified medium, J. Acoust. Soc. Am., 37, (1965).

[58] T. Poole and G. V. Frisk, Locating a sound source of unstable frequency by use of phase tracking J. Acoust. Soc. Am., 109 (5 pt. 2), 2318 (2001).

[59] M. B. Porter, The KRAKEN normal mode program. Rep. SM-245, (SACLANT Undersea Research Centre, La Spezia, Italy, 1991).

[60] Matab: The Language of Technical Computing. (Mathworks, Inc., Framingham, MA) http : //www.mathworks.com

[61] J. C. Preisig and T. F. Duda. Coupled Acoustic Mode Propagation Through Continental-Shelf Internal Solitary Waves. IEEE J. Oceanic Eng., 22, (1997).

[62] S. D. Rajan, J. F. Lynch, and G. V. Frisk, Perturbative inversion methods for obtaining bottom geoacoustic parameters in shallow water. J. Acoust. Soc. Am., 82(3), 998-1017 (1987). 
[63] S. D. Rajan, Waveform inversion for the geoacoustic parameters of the ocean bottom. J. Acoust. Soc. Am., 91(6), 3228-3241 (1991).

[64] S. D. Rajan, Determination of geoacoustic parameters of the ocean bottom data requirements. J. Acoust. Soc. Am., 92(4), 2126-2140 (1992).

[65] S. D. Rajan and S. D. Bhatta, Evaluation of high-resolution frequency estimation methods for determining frequencies of eigenmodes in shallow water acoustic field. J. Acoust. Soc. Am., 93(1), 378-389 (1993).

[66] S. D. Rajan, Waveform Inversion for Geoacoustic Parameters of the Ocean Bottom in Shallow Water: Matched Mode Method. in Full Field Inversion Methods in Ocean and Seismo-Acoustics, edited by O. Diachok, A. Caiti, P. Gerstoft, and H. Schmidt (Kluwer Academic, Dordrecht, 1995), pp. 299-304.

[67] S. D. Rajan, J. A. Doutt, and W. M. Carey, Inversion for the Compressional Wave Speed Profile of the Bottom from Synthetic Aperture Experiments Conducted in the Hudson Canyon Area. IEEE J. Oceanic Eng., 23(03), pp. 174-187 (1998).

[68] S. D. Rajan, personal communication (October 1999).

[69] D. C. Rife and R. R. Boorstyn, Single-Tone Parameter Estimation from DiscreteTime Observations. IEEE Trans. Inform. Theor, IT-20(5), (1974).

[70] D. C. Rife and R. R. Boorstyn, Multiple Tone Parameter Estimation from Discrete-Time Observations. Bell Syst. Tech. Journal, 55(9), (1976).

[71] H. Sakai, Statistical Properties of AR Spectral Analysis. IEEE Trans. Acoust., Speech, Signal Processing, ASSP-27(4), pp. 402-409, (1979).

[72] H. Schmidt, SAFARI: Seismo-acoustic fast field algorithm for range-independent environments. User's Guide. Rep. SR-113 (SACLANT Undersea Research Centre, La Spezia, Italy, 1988). 
[73] H. Schmidt and W. A. Kuperman, Spectral and modal representations of the Doppler-shifted field in ocean waveguides. J. Acoust. Soc. Am., 96(1), (1994).

[74] M. Siderius, P. L. Nielsen, J. Sellschopp, M. Snellen, and D. Simons, Experimental study of geo-acoustic inversion uncertainty due to ocean sound-speed fluctuations. J. Acoust. Soc. Am., 110(2), 769-781, (2001).

[75] K. B. Smith, Benchmarking shallow water range-dependent acoustic propagation modeling. (December 20, 2000).

http : //web.nps.navy.mil/ $\sim$ kbsmith/ChicagoASA/benchmark.html

[76] K. B. Smith, Benchmarking shallow water range-dependent acoustic propagation modeling, Test Case III: Internal Waves. (December 20, 2000). http : //web.nps.navy.mil/ $\sim k b s m i t h / C h i c a g o A S A / i w s . h t m l$

[77] D. C. Stickler and P. A. Deift, Inverse problem for a stratified ocean and bottom. J. Acoust. Soc. Am., 70(6), 1723-1727 (1981).

[78] D. C. Stickler, Inverse scattering in a stratified medium. J. Acoust. Soc. Am., 74(3), 994-1005 (1983).

[79] J. R. Taylor, An Introduction to Error Analysis: The Study of Uncertainties in Physical Measurements. (University Science Books, Mill Valley, CA, 1982).

[80] I. Tolstoy and C. S. Clay, Ocean acoustics; theory and experiment in underwater sound. (McGraw Hill, Inc., New York 1966).

[81] Various Authors, Acoustical Oceanography and Underwater Acoustics: Benchmarking Range-Dependent Numerical Models, chaired by K. B. Smith and A. I. Tolstoy, J. Acoust. Soc. Am., 109 (5 pt. 2), pp. 2332-2335 (2001).

[82] M. West, R. Prado, and A. Krystal, Evaluation and comparison of EEG traces: Latent structure in non-stationary time series. J. Amer. Stat. Assoc., 94, (1999). 
[83] W. D. Wilson, Equation for the speed of sound in sea-water, J. Acoust. Soc. Am., 32(10), (1960). 